\title{
WestVirginiaUniversity
}

THE RESEARCH REPOSITORY @ WVU

Graduate Theses, Dissertations, and Problem Reports

2012

\section{Artificial Intelligence Assisted History Matching - Proof of Concept}

Alireza Shahkarami

West Virginia University

Follow this and additional works at: https://researchrepository.wvu.edu/etd

\section{Recommended Citation}

Shahkarami, Alireza, "Artificial Intelligence Assisted History Matching -- Proof of Concept" (2012).

Graduate Theses, Dissertations, and Problem Reports. 3342.

https://researchrepository.wvu.edu/etd/3342

This Thesis is protected by copyright and/or related rights. It has been brought to you by the The Research Repository @ WVU with permission from the rights-holder(s). You are free to use this Thesis in any way that is permitted by the copyright and related rights legislation that applies to your use. For other uses you must obtain permission from the rights-holder(s) directly, unless additional rights are indicated by a Creative Commons license in the record and/ or on the work itself. This Thesis has been accepted for inclusion in WVU Graduate Theses, Dissertations, and Problem Reports collection by an authorized administrator of The Research Repository @ WVU. For more information, please contact researchrepository@mail.wvu.edu. 
By

\author{
Alireza Shahkarami \\ Thesis submitted to the \\ College of Engineering and Mineral Resources \\ At West Virginia University \\ In partial fulfillment of the requirements \\ for the degree of \\ Master of Science \\ in \\ Petroleum and Natural Gas Engineering
}

Approved by

Shahab D. Mohaghegh, PhD., Chair

Samuel Ameri, M.S.

Grant Bromhal, PhD.

Jitendra Kikani, PhD.

Department of Petroleum and Natural Gas Engineering

Morgantown, West Virginia

2012

Keywords: History Matching, Artificial Intelligence, Surrogate Reservoir Model, AI Assisted History Matching

Copyright 2012 Alireza Shahkarami 


\section{Abstract}

\section{AI Assisted History Matching- Proof of Concept}

\section{Alireza Shahkarami}

History matching is the process of adjusting uncertain reservoir parameters until an acceptable match with the measured production data is obtained. Complexity and sub-optimal knowledge of reservoir characteristics makes this process time-consuming with high computational cost. In the past, computer-assisted history matching has attempted to make this process faster; however, the degree of success of these techniques continues to be a subject for debate.

In this study, the objective is to prove and examine the application of a relatively new Artificial Intelligence based technology (Surrogate Reservoir Model - SRM) to assist the history match process. SRM is a prototype of full-field reservoir simulation model that runs in fractions of a second. The capability of generating meaningful outputs in a short time period with acceptable accuracy makes SRM a unique tool for assisted history matching.

In this project, an SRM was created for a synthetic case study of a heterogeneous and complex oil field, with 24 production wells and 30 years of production history. The history matching was performed for this field using SRM and tuning static data (permeability). The result of this study is a proof of concept and shows that SRM is able to reproduce the numerical simulator results faster and with an acceptable accuracy. These characteristics make SRM a fast and effective tool for assisted history matching. 


\section{Acknowledgement}

I would like to show my gratitude and appreciation to my research advisor Dr. Shahab Mohaghegh for his advice, guidance, and encouragement during the course of this research.

My appreciation goes to Prof. Samuel Ameri, Dr. Razi Gaskari, Dr. Grant Bromhal and Dr. Jitendra kikani, who kindly accepted to be a member of my thesis committee and all made significant contribution to this work through their generous comments.

I wish to thank my friends and colleagues at PEARL group for providing a friendly environment and helpful discussions about different issues during the past two years. I also wish to thank all my friends; ones who have been beside me and ones who are distant, without their support studying all long days and nights far away from home would not be ever possible.

In addition, I would like to express my gratitude to Computer Modeling Group and Intelligent Solution Inc., for making the CMG reservoir simulator, IDEA and IMAGINE software available to me to perform the reservoir modeling in this work.

My profound gratitude is expended to my parents. Although they have been miles away from me, I have always been supported by their understanding, trust and wholehearted help they have been giving to me. 


\section{Table of Contents}

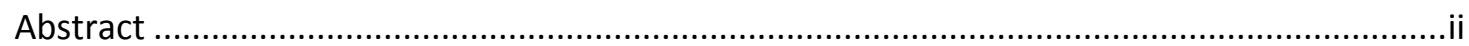

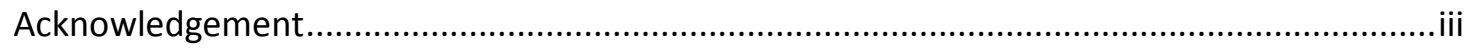

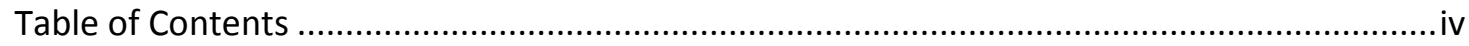

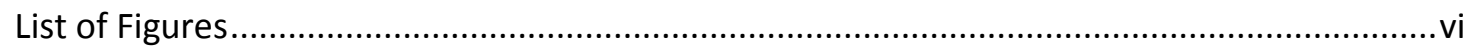

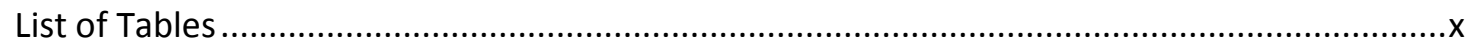

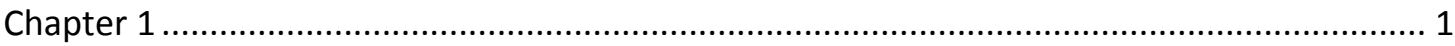

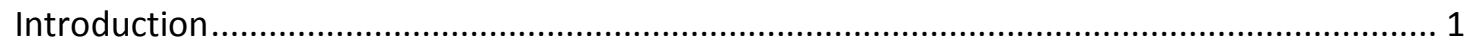

1.1 Introduction to Reservoir Simulation and Modeling ........................................................ 1

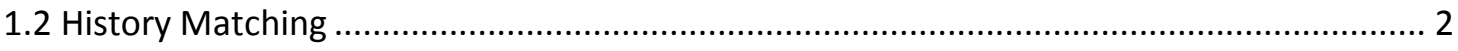

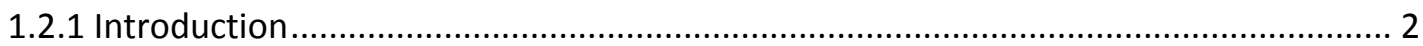

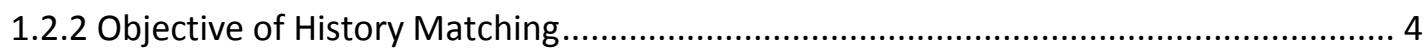

1.2.3 Performance Data and Selecting Adjustable Parameters ......................................... 4

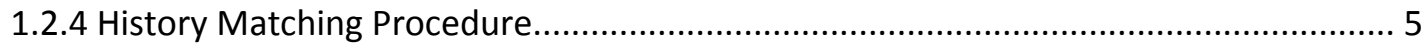

1.3 Computer Assisted or Automated History Matching ...................................................... 8

1.4 Application of Neural Networks in petroleum engineering .......................................... 11

1.5 Artificial Intelligence (Al) assisted History Matching ...................................................... 13

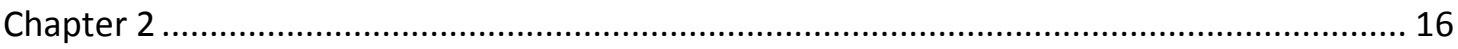

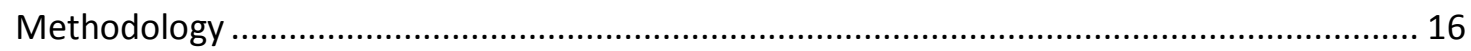

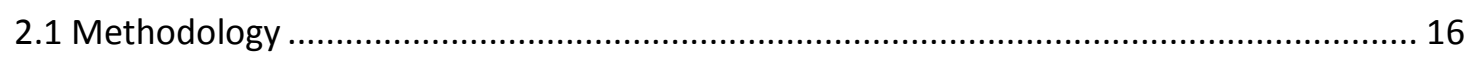

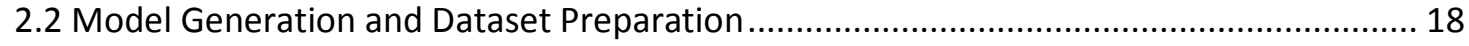

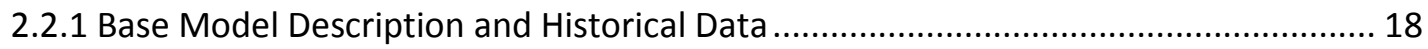

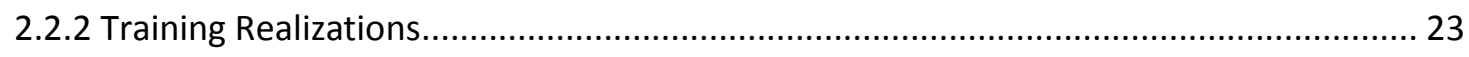

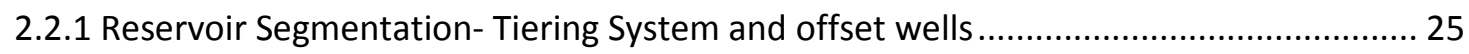

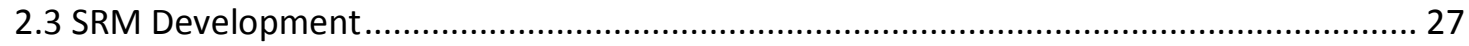

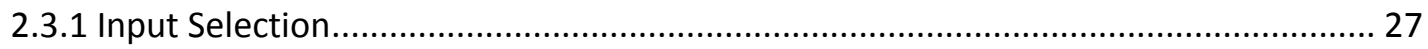

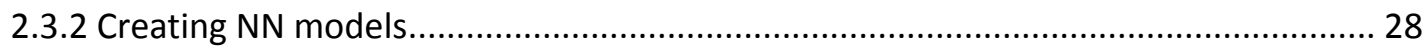

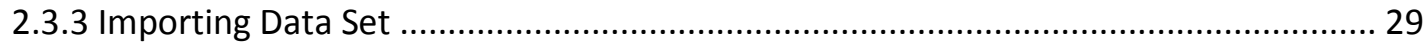

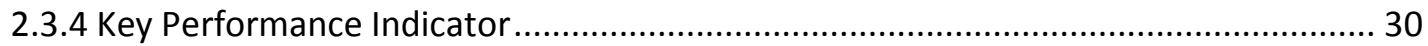

2.3.5 Training, Calibration and Verification Sets of Data .................................................. 34 


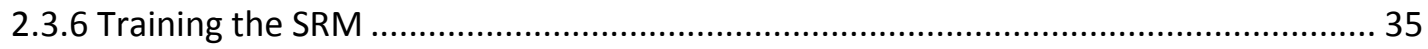

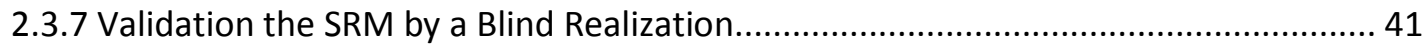

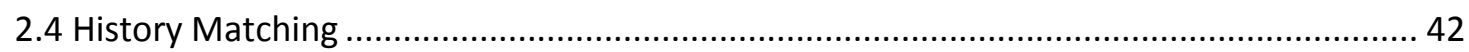

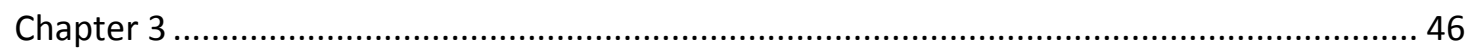

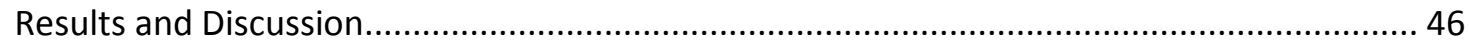

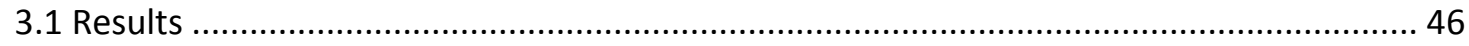

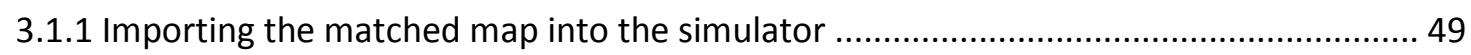

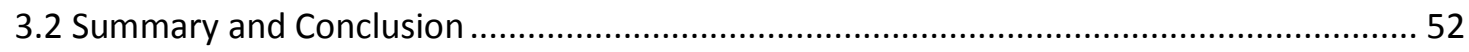

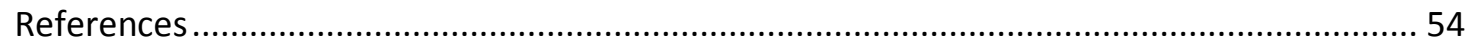

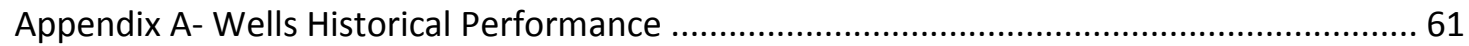

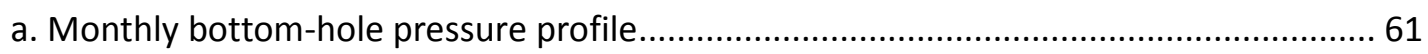

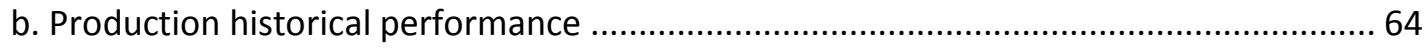

Appendix B- 10 Created permeability distributions for training SRM ................................... 67

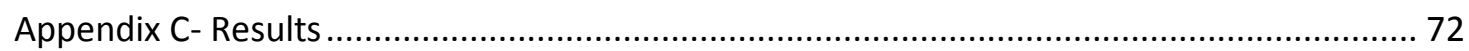

a. SRM results for training, calibration and verification using 10 realizations:................ 72

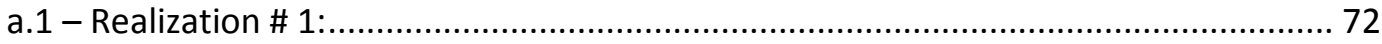

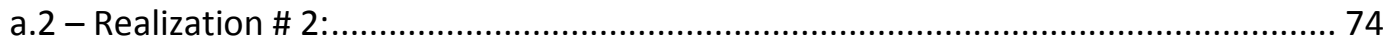

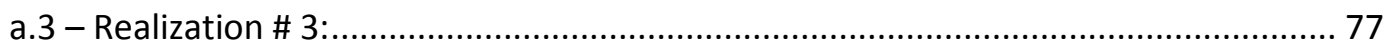

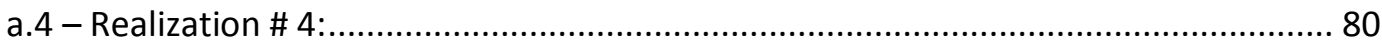

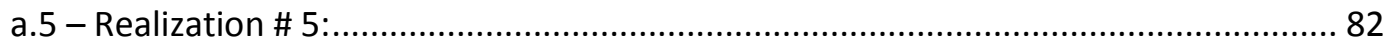

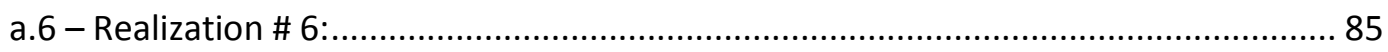

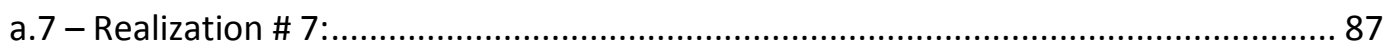

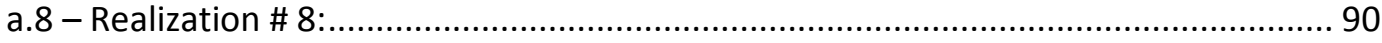

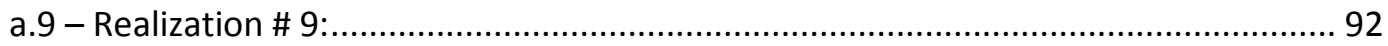

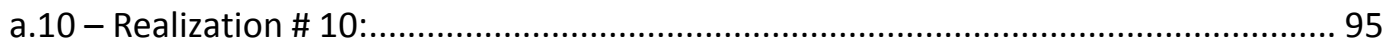

b. Validation of SRM using blind verification realization.............................................. 98

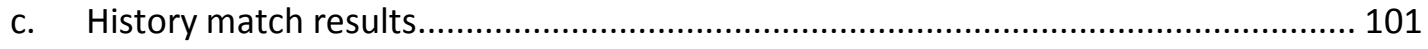

d. Results of simulator using matched permeability distribution ................................. 104 


\section{List of Figures}

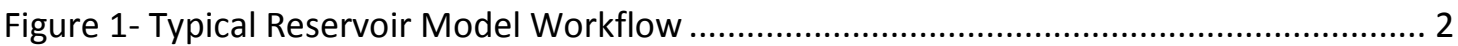

Figure 2- Matching pressure behavior (Cosentino, 2001) (Toronyi \& Saleri, 1988).................. 7

Figure 3- Matching saturation performance of reservoir (Toronyi \& Saleri, 1988) (Cosentino,

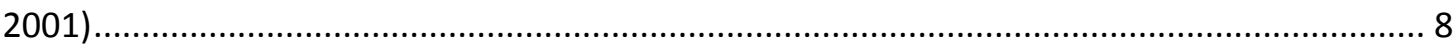

Figure 4- Some famous Assisted History Matching Tools in the industry ............................... 10

Figure 5- An artificial neural network is an interconnected group of nodes ............................ 12

Figure 6- A diagram describing different steps in the project............................................... 18

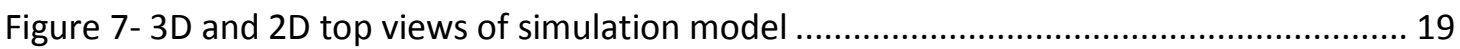

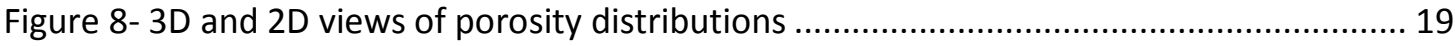

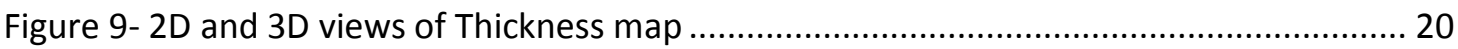

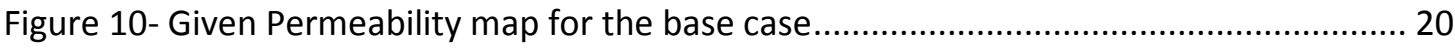

Figure 11- Geological information identifies a high permeable zone in the field.................... 21

Figure 12- Bottom-hole pressure as constraints for the production wells ............................ 21

Figure 13- Production Historical data for some wells, rest of wells are available in Appendix A

Figure 14- Different permeabilty distributions created to train SRM (the rest is available in

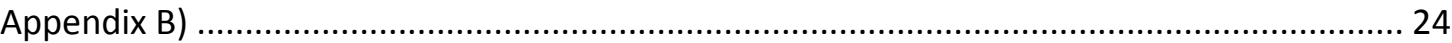

Figure 15- The reservoir divided to different drainage areas following Voronoi diagram ...... 26

Figure 16- Different tiers were designed based on the Voronoi diagram.............................. 26

Figure 17- Different types of data in the spatio-temporal database ....................................... 28

Figure 18- Attribute Selection for Imported Data Set ............................................................ 29

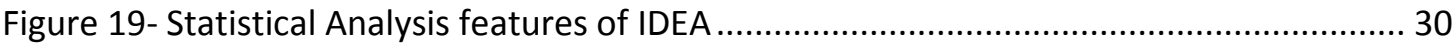

Figure 20- KPI Analysis, tornado plot for the Initialization model......................................... 31

Figure 21- KPI Analysis, tornado plot for the Main NN model ............................................... 32

Figure 22- The Initialization NN model structure with 20 neurons in the hidden layer .......... 36

Figure 23- The Main NN model structure with 50 neurons in the hidden layer ...................... 37

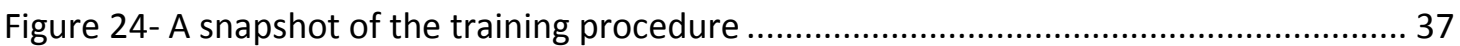

Figure 25-Scatter plot of Training set results in the Initialization Model................................... 38

Figure 26- Scatter plot of Calibration set results in the Initialization Model .......................... 39

Figure 27-Scatter plot of Verification set results in the Initialization Model ........................... 39

Figure 28- Scatter plot of Training set results in the Main Model ........................................... 39

Figure 29-Scatter plot of Calibration set results in the Main Model....................................... 40

Figure 30-Scatter plot of Verification set results in the Main Model................................... 40

Figure 31-3D view of permeability map for the blind verification realization ......................... 41

Figure 32- 2D view of permeability map for the blind verification realization ........................ 42

Figure 33- A flowchart explaining the manual history match process in this study ................ 44

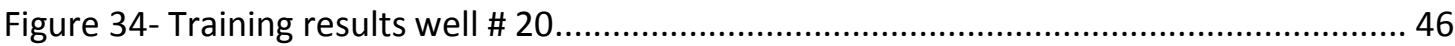

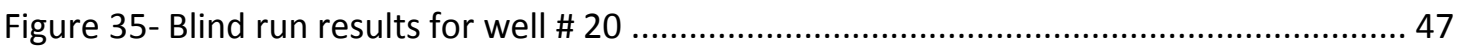

Figure 36- SRM-Based History Matching Results for well\# 20 ............................................. 47 
Figure 37- comparison of matched and actual Permeability distributions ............................. 48

Figure 38- A snapshot of error distribution of permeability.

Figure 39 - Comparison of field cumulative production between actual data and the simulator performance. The permeability map is the matched permeability map coming from the SRM.

Figure 40-Comparison of oil rate production between actual data and the results coming from simulator after applying the matched permeability map from SRM

Figure 41-Comparison of cumulative production between actual data and the results coming

from simulator after applying the matched permeability map from SRM ............................ 50

Figure 42- Error distribution for history matched results ........................................................ 51

Figure 43- Error frequency distribution for the history match results................................. 51

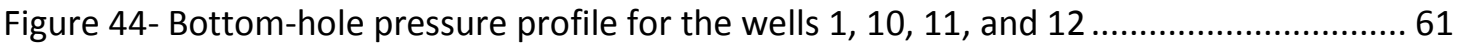

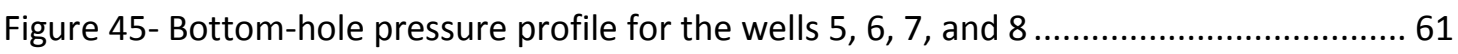

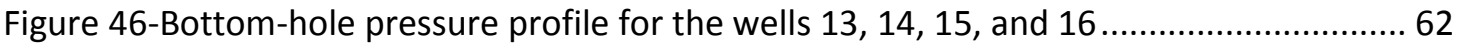

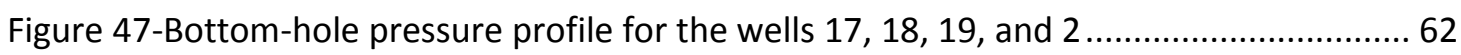

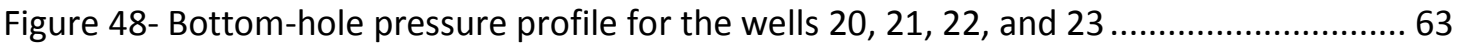

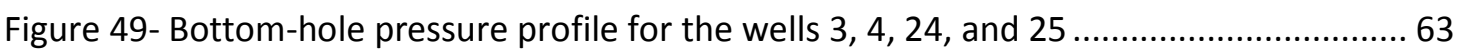

Figure 50- Oil rate and cumulative production for the wells 1, 10, 11 and $12 \ldots \ldots \ldots \ldots \ldots \ldots \ldots . . . . . . . .64$

Figure 51- Oil rate and cumulative production for the wells $13,14,15$ and 16 ..................... 64

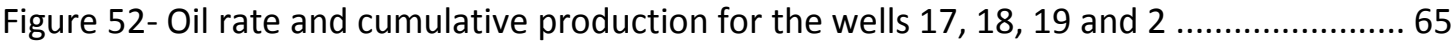

Figure 53- Oil rate and cumulative production for the wells 20, 21, 22 and 23 ................... 65

Figure 54- Oil rate and cumulative production for the wells $24,25,3$ and $4 \ldots \ldots \ldots \ldots \ldots \ldots \ldots \ldots . . . . . . . . . . .66$

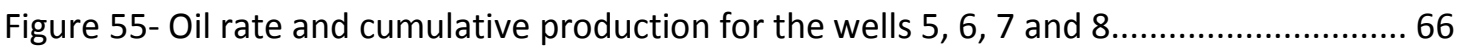

Figure 56- Permeability Map for different Training Realizations- Run\#1............................... 67

Figure 57- Permeability Map for different Training Realizations- Run\#2 ................................ 67

Figure 58- Permeability Map for different Training Realizations- Run\#3.................................6 68

Figure 59-Permeability Map for different Training Realizations- Run\#4 ............................... 68

Figure 60-Permeability Map for different Training Realizations- Run\#5 ............................... 69

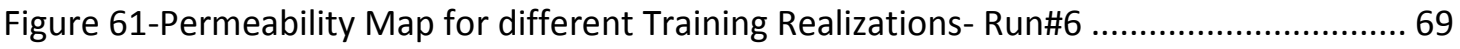

Figure 62-Permeability Map for different Training Realizations- Run\#7 .............................. 70

Figure 63-Permeability Map for different Training Realizations- Run\#8 ............................... 70

Figure 64-Permeability Map for different Training Realizations- Run\#9 ................................ 71

Figure 65-Permeability Map for different Training Realizations- Run\#10 ............................... 71

Figure 66- Comparison of the SRM results with simulator outputs after the training process-

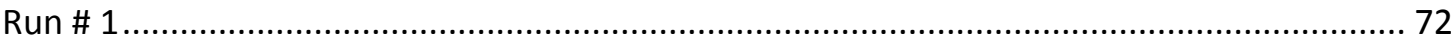

Figure 67- Comparison of the SRM results with simulator outputs after the training process-

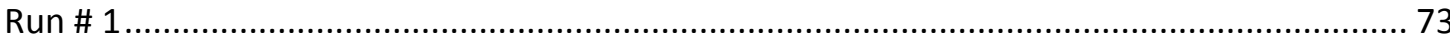

Figure 68-Comparison of the SRM results with simulator outputs after the training process-

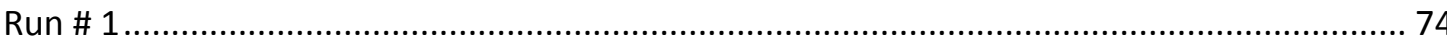

Figure 69- Comparison of the SRM results with simulator outputs after the training process-

Run \# 2 
Figure 70- Comparison of the SRM results with simulator outputs after the training processRun \# 2

Figure 71- Comparison of the SRM results with simulator outputs after the training process-

Run \# 2

Figure 72- Comparison of the SRM results with simulator outputs after the training process-

Run \# 2

Figure 73- Comparison of the SRM results with simulator outputs after the training process-

Run \# 3

Figure 74- Comparison of the SRM results with simulator outputs after the training process-

Run \# 3

Figure 75- Comparison of the SRM results with simulator outputs after the training process-

Run \# 3

Figure 76- Comparison of the SRM results with simulator outputs after the training process-

Run \# 4

Figure 77- Comparison of the SRM results with simulator outputs after the training process-

Run \# 4

Figure 78- Comparison of the SRM results with simulator outputs after the training process-

Run \# 4

Figure 79- Comparison of the SRM results with simulator outputs after the training process-

Run \# 5

Figure 80-Comparison of the SRM results with simulator outputs after the training process-

Run \# 5

Figure 81- Comparison of the SRM results with simulator outputs after the training process-

Run \# 5

Figure 82- Comparison of the SRM results with simulator outputs after the training process-

Run \# 6 . 85

Figure 83-Comparison of the SRM results with simulator outputs after the training process-

Run \# 6 . 86

Figure 84- Comparison of the SRM results with simulator outputs after the training process-

Run \# 6 .

Figure 85- Comparison of the SRM results with simulator outputs after the training process-

Run \# 7

Figure 86-Comparison of the SRM results with simulator outputs after the training process-

Run \# 7

Figure 87- Comparison of the SRM results with simulator outputs after the training process-

Run \# 7

Figure 88- Comparison of the SRM results with simulator outputs after the training process-

Run \# 8

Figure 89- Comparison of the SRM results with simulator outputs after the training process-

Run \# 8 .

Figure 90- Comparison of the SRM results with simulator outputs after the training process-

Run \# 8 . 
Figure 91- Comparison of the SRM results with simulator outputs after the training processRun \# 9.

Figure 92- Comparison of the SRM results with simulator outputs after the training processRun \# 9 .

Figure 93- Comparison of the SRM results with simulator outputs after the training processRun \# 9. 94

Figure 94- Comparison of the SRM results with simulator outputs after the training processRun \# 10 95

Figure 95- Comparison of the SRM results with simulator outputs after the training processRun \# 10 96

Figure 96-Comparison of the SRM results with simulator outputs after the training processRun \# 10

Figure 97- Comparison of the SRM results with simulator outputs, the blind verification run, wells 5, 17, 18 and 20 . 98

Figure 98-Comparison of the SRM results with simulator outputs, the blind verification run, wells $13,14,16$ and 25

Figure 99- Comparison of the SRM results with simulator outputs, the blind verification run, wells $3,4,7$ and 12

Figure 100-Comparison of the SRM results with simulator outputs, the blind verification run,

wells $19,21,22$ and 23 99

Figure 101- Comparison of the SRM results with simulator outputs, the blind verification run, wells $6,8,15$ and 24 100

Figure 102-Comparison of the SRM results with simulator outputs, the blind verification run, wells $1,2,10$ and 11 100 Figure 103-Comparison of the SRM results with field data, history match results; wells 1, 2, 3 and 4 101

Figure 104- Comparison of the SRM results with field data, history match results; wells 5, 6, 12 and 13 101

Figure 105- Comparison of the SRM results with field data, history match results; wells 7, 8,

10 and 11 102

Figure 106- Comparison of the SRM results with field data, history match results; wells 14,16 , 24 and 25

Figure 107- Comparison of the SRM results with field data, history match results; wells 15,18 , 19 and 20

Figure 108- Comparison of the SRM results with field data, history match results; wells 17, 21, 22 and 23 103

Figure 109- Comparison of oil rate production between actual data and the results coming from simulator after applying the matched permeability map from SRM 104

Figure 110-Comparison of oil rate production between actual data and the results coming from simulator after applying the matched permeability map from SRM ... 104

Figure 111- Comparison of oil rate production between actual data and the results coming from simulator after applying the matched permeability map from SRM 105 
Figure 112-Comparison of oil rate production between actual data and the results coming from simulator after applying the matched permeability map from SRM 105

Figure 113-Comparison of oil rate production between actual data and the results coming from simulator after applying the matched permeability map from SRM 106

Figure 114- Comparison of oil rate production between actual data and the results coming from simulator after applying the matched permeability map from SRM 106

Figure 115-Comparison of cumulative production between actual data and the results coming from simulator after applying the matched permeability map from SRM 107

Figure 116-Comparison of cumulative production between actual data and the results coming from simulator after applying the matched permeability map from SRM 107

Figure 117-Comparison of cumulative production between actual data and the results coming from simulator after applying the matched permeability map from SRM 108

Figure 118-Comparison of cumulative production between actual data and the results coming from simulator after applying the matched permeability map from SRM 108

Figure 119-Comparison of cumulative production between actual data and the results coming from simulator after applying the matched permeability map from SRM 109 Figure 120-Comparison of cumulative production between actual data and the results coming from simulator after applying the matched permeability map from SRM 109

\section{List of Tables}

Table 1- Data Required for a Simulation Study, (Fanchi, 2006) 3

Table 2- Porosity, Thickness and Permeability values at the Wells Locations 22

Table 3- Different permeability values at wells' locations for creating permeability maps .... 24

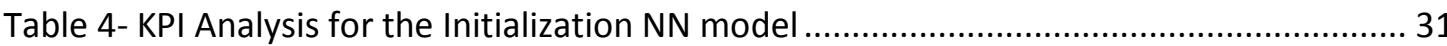

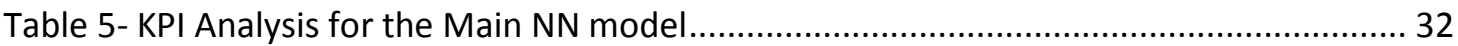

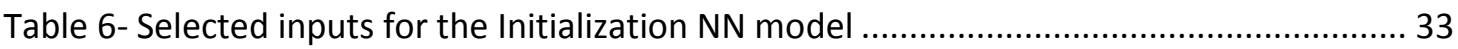

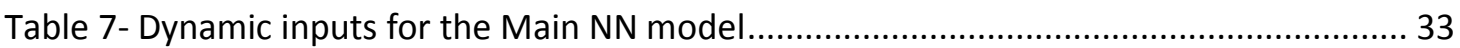

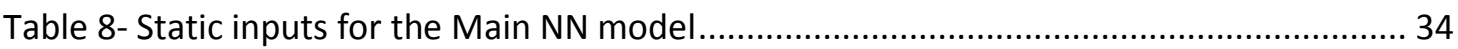




\section{Chapter 1 Introduction}

\subsection{Introduction to Reservoir Simulation and Modeling}

Reservoir simulation is an area of reservoir engineering, which uses computer models to predict the flow of fluids (typically, oil, water, and gas) through porous media. Reservoir simulation is an assimilation of physics, mathematics, reservoir engineering, and computer programming, and its objective is building a tool to predict interaction between hydrocarbon and reservoir under different operating conditions.

Reservoir simulation began in 1954 with the radial gas-flow computations (Watts, 1997). Since then, advancements in computer technologies have resulted in tremendous improvements in reservoir simulation. During these years, advancements happened in model size, technical methods and user communities. These all changed the role of reservoir simulation in industry and business. In addition, the introduction of high-speed computers and electronic resources in the last two decades have changed reservoir simulation from a fancy and costly approach to a practical toolbox, which could be available on the laptop of a student. Today, although reservoir simulation is an established technology, it continues to improve. Nowadays, results of reservoir simulation models are used in almost all reservoir development decisions. Simulating reservoirs easily and realistically makes them a primary and reasonable choice for oil and gas companies in the development of new (green) fields. Similarly, they are used in developed (brown or mature) fields where production forecasts are needed to help make future investment decisions. 
A reservoir simulation model provides means to incorporate geology, reservoir and fluid characteristics into a cohesive computer model in order to calculate pressure and saturation changes throughout the reservoir and predict fluid rate and pressure profile at the wellbore.

A typical reservoir model is used to accomplish the following tasks:

- Sensitivity analysis of uncertain parameters

- Conditioning a simulation model to history data (History Matching)

- Field-development planning and production optimization

- Probabilistic forecasting and risk analysis (Uncertainty Assessment)

Figure 1 shows the workflow for the reservoir simulation process.

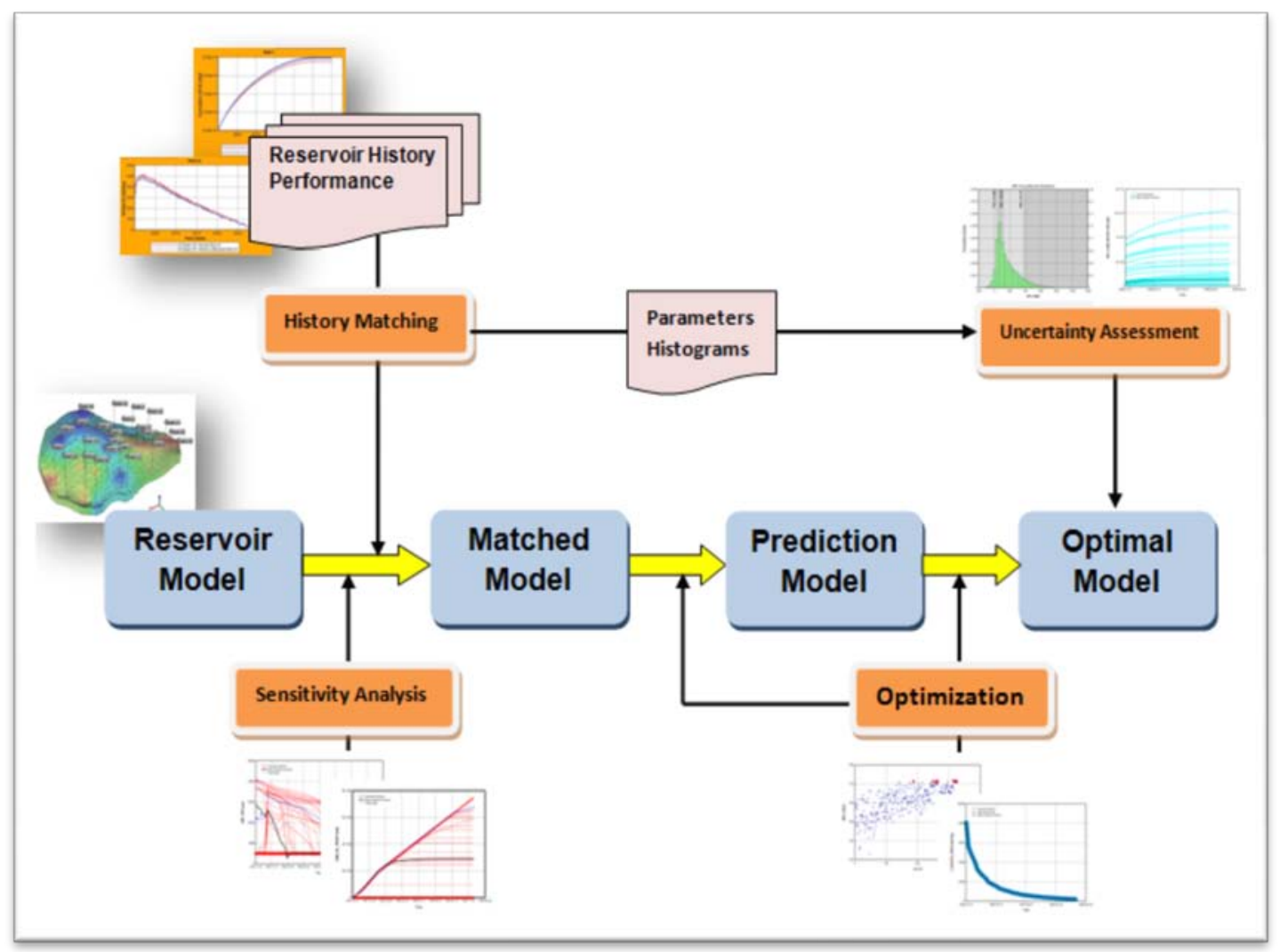

Figure 1- Typical Reservoir Model Workflow

\subsection{History Matching}

\subsubsection{Introduction}

Engineers face different degrees of limitation to build a simulation model. One limitation is lack of data in order to create the simulation model. Table 1 summarizes the essential data to create a simulation model (Fanchi, 2006). The techniques to acquire these data mostly are well 
testing, well logs, core analyses, laboratory studies of reservoir fluids and wellbore performance. Compared to the size of a reservoir, these data are inadequate and their sources represent a limited portion of the reservoir. However, these data and techniques are the only tools that provide input data for the reservoir simulation model of the field under study. The sources and techniques for preparing input data have been reviewed extensively in reservoir simulation literature (Ertekin, Abou-Kassem, \& King, 2001). The main point is that a large part of the reservoir remains unknown to the engineers and geologist working on the simulation model. As a result, the initial simulation model needs adjustment to match the available historical production data and predict the future performance of reservoir. This tuning procedure is performed during history matching process. Thus, history matching is a calibration process that includes adjusting uncertain parameters of a reservoir model until the model reproduces the historical field performance as closely as possible.

Table 1- Data Required for a Simulation Study, (Fanchi, 2006)

\begin{tabular}{|c|c|}
\hline Property & Sources \\
\hline Permeability & $\begin{array}{l}\text { Pressure transient testing, Core analyses, } \\
\text { Correlations, Well performance }\end{array}$ \\
\hline Porosity, Rock compressibility & Core analyses, Well logs \\
\hline $\begin{array}{l}\text { Relative permeability and capillary } \\
\text { pressure }\end{array}$ & Laboratory core flow tests \\
\hline Saturations & $\begin{array}{l}\text { Well logs, Core analyses, Pressure cores, } \\
\text { Single well tracer tests }\end{array}$ \\
\hline Fluid property (PVT) data & $\begin{array}{l}\text { Laboratory analyses of reservoir fluid } \\
\text { samples }\end{array}$ \\
\hline Faults, boundaries, fluid contacts & Seismic, Pressure transient testing \\
\hline Aquifers & $\begin{array}{l}\text { Seismic, Material balance calculations, } \\
\text { Regional exploration studies }\end{array}$ \\
\hline $\begin{array}{l}\text { Fracture spacing, orientation, } \\
\text { connectivity }\end{array}$ & $\begin{array}{l}\text { Core analyses, Well logs, Seismic, Pressure } \\
\text { transient tests, Interference testing, } \\
\text { Wellbore performance }\end{array}$ \\
\hline $\begin{array}{l}\text { Rate and pressure data, completion and } \\
\text { workover data }\end{array}$ & Field performance history \\
\hline
\end{tabular}




\subsubsection{Objective of History Matching}

The main objective of history matching is to improve and validate the reservoir simulation model by incorporating the observed data into the characterization process, in order to obtain reliable production forecasting (Maschio \& Schiozer, 2005). Generally, the initial reservoir model does not match the historical data well enough to use it confidently for future forecasts (Ertekin, Abou-Kassem, \& King, 2001) (Chen Z. , 2007). By having a verified reservoir model coming from history match process, prediction of future performance has a higher degree of confidence. Moreover, once the most uncertain reservoir parameters have been estimated by matching observed reservoir behavior, the tuned model can be used to predict the performance of the reservoir under different operating scenarios.

On the other hand, a quality history match has a potential to better capture the production mechanisms in the reservoir. Therefore, there is a chance to improve the future development plans of the reservoir and modify the different reservoir characterization and data acquisition programs (Ertekin, Abou-Kassem, \& King, 2001). These data will help improve the reservoir model and consequently history match, model forecasts, and general understanding of the reservoir. Another beneficial consequence of a well-done history match is to reconcile certain observations in the field. This kind of behavior could be due to unusual operating constraints or some hidden reservoir properties. For example, problems such as casing leaks, improper allocation of fluids to wells etc (Mattax \& Dalton, 1990) could be identified during a history matching process.

\subsubsection{Performance Data and Selecting Adjustable Parameters}

In general, the reservoir performance data include production and pressure data coming from all wells (injection, production or observation). Usually for a given production schedule the matching data are (Mattax \& Dalton, 1990) (Chen Z. , 2007):

- Oil, water and gas production rates

- Average pressures (shut-in pressures) or pressures at observation wells

- Flowing well pressures

The historical data are checking points in a matching process; as a result, having a precise and reliable historical data is very crucial in the history match process. Untrustworthy data will mislead the matching procedure and the results of history match process will be different from the actual. This will result in having an unreliable simulation model, which gives wrong prediction of reservoir performance. For example in the past, gas production measurements 
were not precise, especially if gas was flared. Usually oil production measurements are more accurate. For injection data, there is a chance to lose fluids in other layers or leaking can happen in the casing or behind the pipe (Mattax \& Dalton, 1990).

The quality and quantity of available historical data and the quality of initial reservoir model affects the history match process. Having reliable knowledge of geological properties of the reservoir will decrease the uncertainty involved in the process of tuning model parameters. Performing sensitivity analysis as a pre-history matching process is giving some ideas about the sensitive parameters in the model and can save some time during history matching. As previously mentioned, the calibrated model is used to forecast the future performance of the reservoir with a higher degree of confidence. The process could be repeated as new production and pressure data become available.

A primary step in history matching process is identifying the uncertain parameters. In practice, some parameters such as well locations, hydrocarbon PVT parameters, oil flow rates, gross thickness, initial pressure, and net-to-gross cutoff values are known with confidence and not adjusted to get a match (Cosentino, 2001). In case of having aquifer in the field, some parameters such as aquifer transmissibility, storage, and strength are candidates to be changed in order to get a match. In a reservoir, following parameters are the most uncertain parameters and they can be adjusted to achieve a calibrated model: reservoir permeability, porosity, thickness, relative permeability, capillary pressure functions, rock properties such as compressibility as well as reservoir fluid properties and their distribution, water properties, WOC, and GOC, (Mattax \& Dalton, 1990).

In order to history match a model, adjustments are usually needed in the parameters (e.g., pore volume and net to gross ratio, contact levels, capillary pressure) that influence the volume of original oil in place (OOIP). However, the final OOIP from dynamic modeling should be in an acceptable range with calculated from the geological model, material balance and/or decline curve analysis (Cosentino, 2001).

\subsubsection{History Matching Procedure}

History matching is a complicated task. The following criteria determine the degree of success of this process: the quality and quantity of available data, the specific characteristic of the reservoir under study, the time and resources allocated to the study, and finally the experience and knowledge of the group working on the model. Consequently, each one of these criteria give the history matching problem its most important characteristic which is the non- 
uniqueness character of the solution. There is no specific and unique method for history matching process. Each reservoir has a particular specification and behavior. Geological characterization, performance history, production development and operations, reservoir drive mechanism, and wells' specifications are the properties that make each field unique (Cosentino, 2001).

Numerous literatures have described a systematic approach to perform history matching (Mattax \& Dalton, 1990) (Cosentino, 2001) (Ertekin, Abou-Kassem, \& King, 2001), therefore in this section we are not trying to go through the details of a history match process.

Identifying the most uncertain parameters, which at the same time have a significant impact on the reservoir performance, is an initial step in any simulation process. Mostly these parameters are determined based on the type of the reservoir; however, some parameters such as permeability due to their effect on the fluid flow in the porous media play an important role in all kinds of reservoirs.

Similarly, key wells are playing important role in simulation process. Cosentino (2001), defines key wells as the wells whose production behavior is typical and must be correctly reproduced by the model. In studies that include a limited number of wells, possibly less than 20, all the wells can virtually be considered as key wells and the matching effort can be addressed with the objective of correctly reproducing the behavior of all these wells.

By identifying the critical reservoir properties and defining the key wells, the reservoir engineers working the project have a simplified reference framework to begin the history match process. In a manually performed history match, two general steps can be considered.

- The first step, called pressure match, is trying to get a match for pressure behavior of the field. At this step, the objective is adjusting the global energy balance. Figure 2, (Toronyi \& Saleri, 1988) (Cosentino, 2001) describes the procedure at this level. As it was mentioned, permeability is a vital parameter affecting flow in the reservoir. Therefore, permeability could be modified globally and locally to achieve the pressure match. 


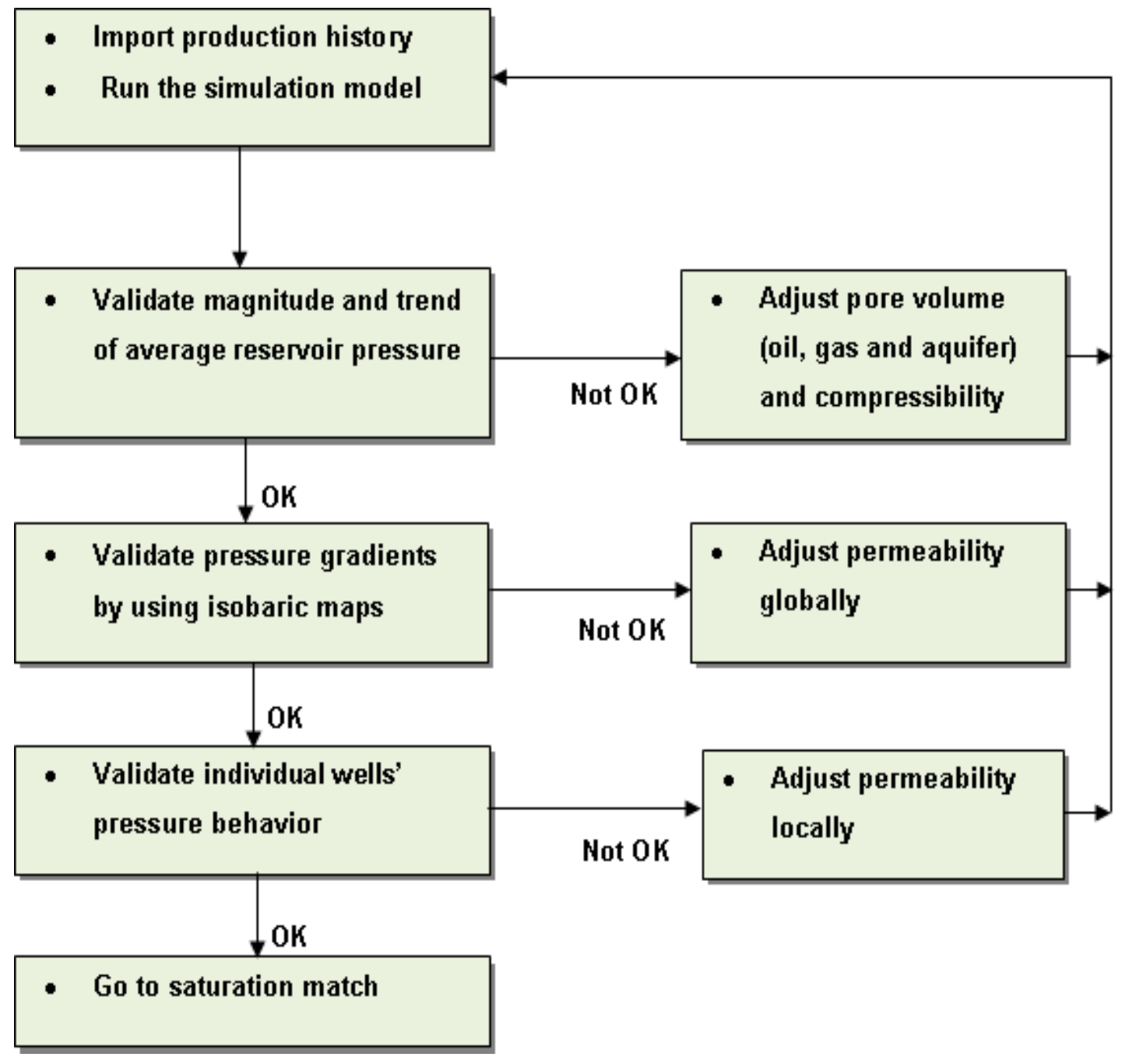

Figure 2- Matching pressure behavior (Cosentino, 2001) (Toronyi \& Saleri, 1988)

- The second phase, called saturation match, is trying to match the fluid saturation in the reservoir. In the same way, permeability as well as relative permeability curves and functions are the critical parameters in order to get a match. Figure 3 demonstrates the steps to get a saturation match. 


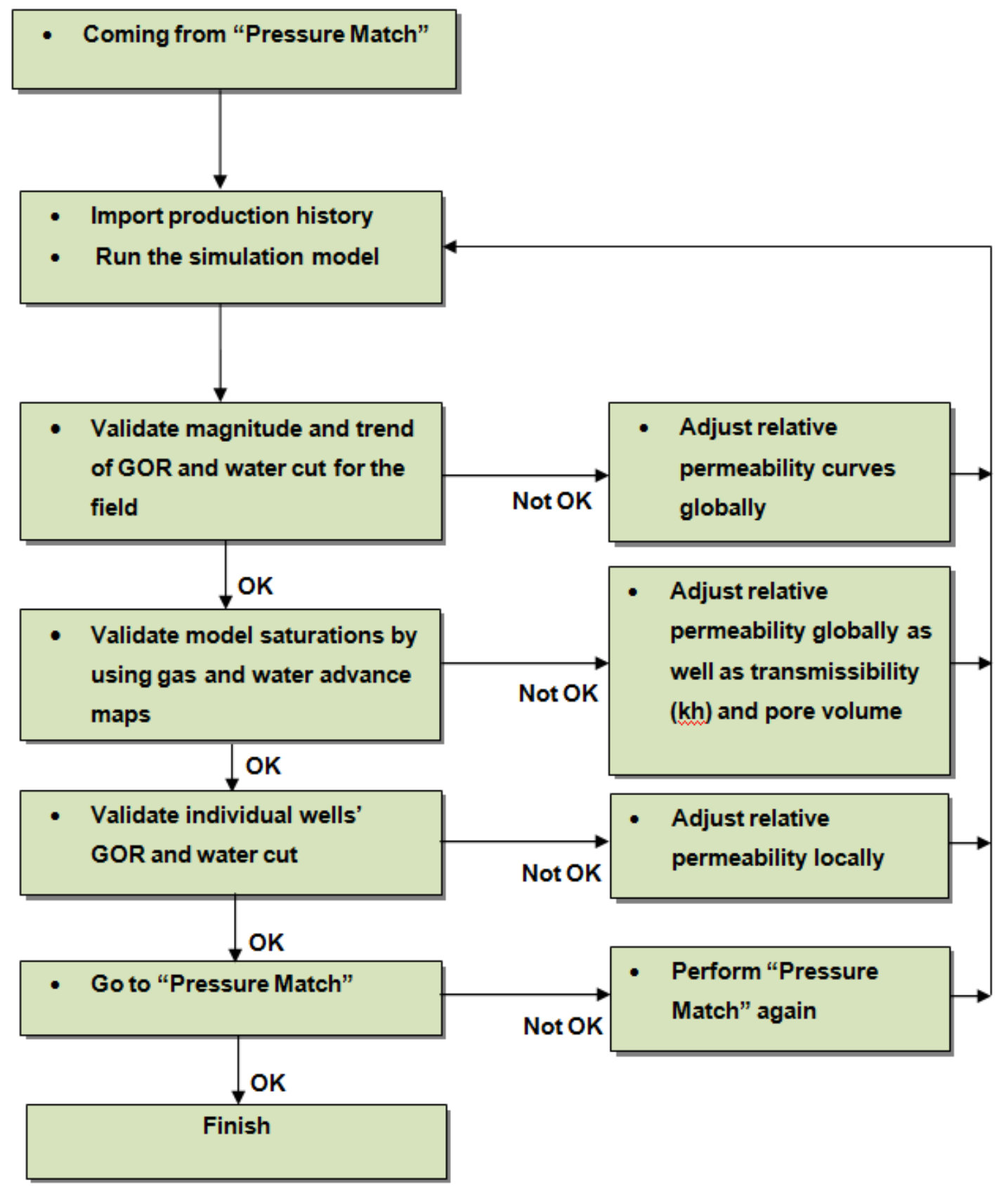

Figure 3- Matching saturation performance of reservoir (Toronyi \& Saleri, 1988) (Cosentino, 2001)

\subsection{Computer Assisted or Automated History Matching}

History matching, by nature, is an ill-posed inverse problem. Correspondingly, classical history matching where reservoir parameters are adjusted manually by trial-and-error makes this process more tedious and time consuming. As a result, automatic history matching was proposed to decrease the amount of labor-intensive work in manual history matching. 
During the last decade, there has been a large increase in deployment of permanent down-hole gauges (PDGs) and as a result acquisition of real-time data. The amount of data is beyond the potential of manual history matching; resulting in demands to develop tools that speed up history matching (Kabir, Chien, \& Landa, 2003).

Generally, automated history matching tries to keep the geological model plausibility and estimates some uncertain reservoir properties while trying to match the field performance. The basic process is to start from an initial parameter guess and improve it by integrating field data in an automatic loop. The quality of the match between measured and computed data is generally evaluated by using an objective function.

Many efforts to improve manual history matching techniques have been made since the mid 1960's to both speed up and automate history match process (Kruger, 1961) (Jacquard \& Jain, 1965) (Jahns, 1966). Gradient optimization methods were used for history matching in late 1960’s (Coats, Dempsey, \& Henderson, 1968) (Slater \& Durrer, 1970). Chen et al (1974) tried to formulate history matching as an optimal control problem. Bush and Carter (1996) showed that simple optimization techniques are not good enough to address complex history matching problems. He and Chamber (1999) claimed that automatic history matching using an objectbased approach could provide acceptable results without the need to manually adjust the model.

In the early 90's, stochastic modeling was used to generate multiple realizations (Tyler, Svanes, \& Omdal, 1993) (Palatnic, Zakirov, Haugen, \& van Roosmalen, 1993). Stochastic modeling, which provides many different equi-probable geological realizations, allows variation of the most important input parameters while honoring the underlying available data, e.g. the geological properties influencing fluid flow.

Sultan et al (1994) and Ouenes et al (1993) used the simulated annealing method (SAM) to automate history matching process. SAM was a nongradient optimization method capable of handling large number of parameters.

Gao et al (2004) for the first time suggested the idea of combining the simultaneous perturbation stochastic approximation (SPSA) method with a simulator to perform automatic history match of multiphase flow production data. Hajizadeh et al (2009) (2010) introduced a stochastic approach for automatic history matching based on a continuous Ant Colony Optimization (ACO) algorithm. Other stochastic algorithms have been examined in this area. Following are algorithms applied in order to automate history match process: 
- Neighborhood algorithm (Subbey \& Christie, 2003), Genetic algorithms (Erbas \& Christie, 2007) (Castellini, 2005), Scatter search (Sousa, 2007), Tabu search (Yang, Ngheim, \& Card, 2007), Hamiltonian Monte Carlo (HMC) (Mohamed, Christie, \& Demyanov, 2009), Particle Swarm Optimization (PSO) (Mohamed, Christie, \& Demyanov, 2009) (Kathrada, 2009), Markov chain Monte Carlo (Maucec, 2007), and Chaotic optimization (Mantica, 2002).

Nonlinear behavior of the problem, substantial computational expenses and large dimension of a real size field make the process difficult. Although significant computational and solver efficiencies have been gained over the past four decades, ever-increasing size of geo-statistical earth models have continued to challenge the computational speed issue (Kabir, Chien, \& Landa, 2003).

Figure 4 introduces some computer assisted tools have been developed by different major companies.

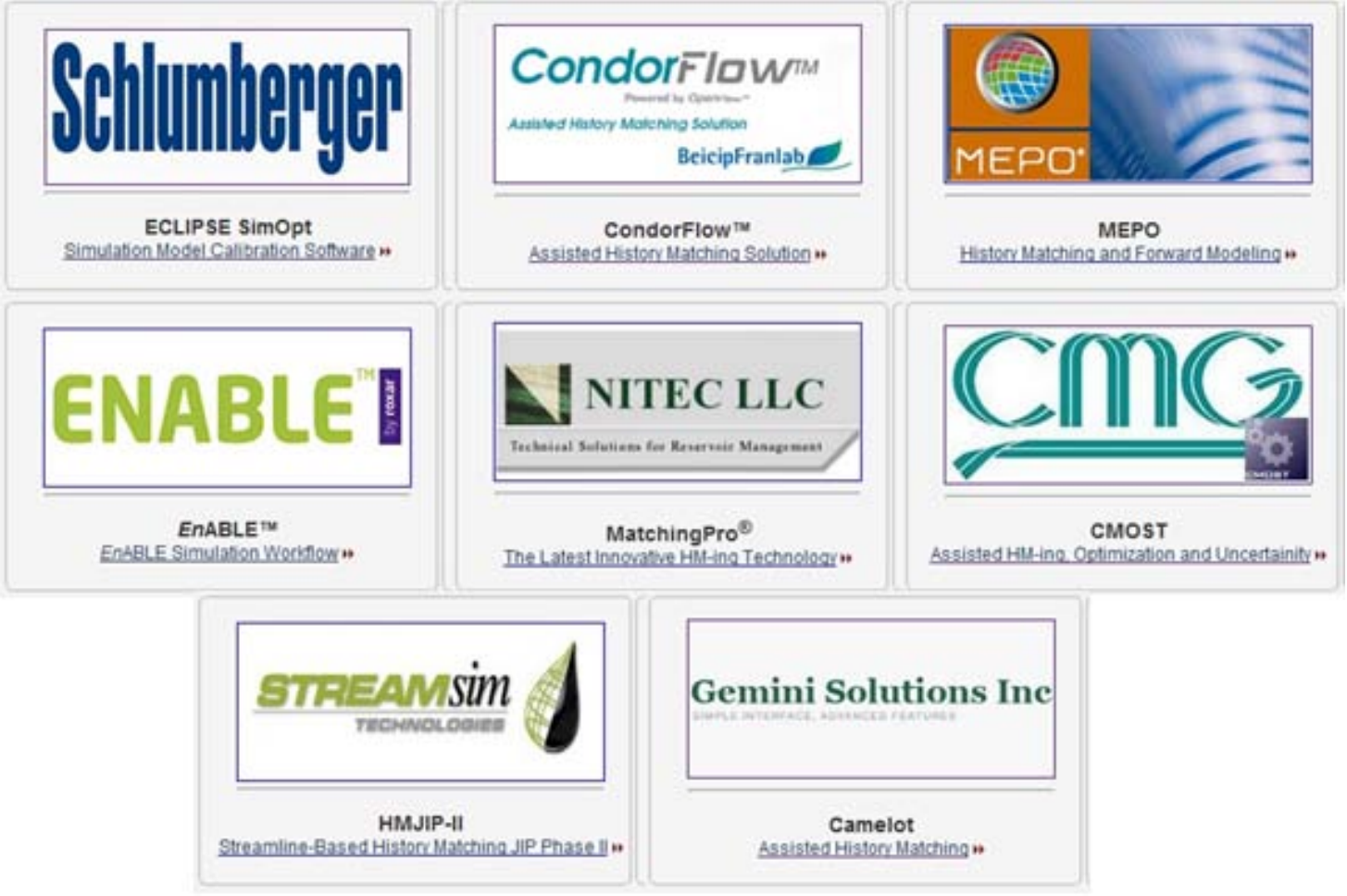

Figure 4- Some famous Assisted History Matching Tools in the industry

Although assisted history matching could be a rapid way to handle parameter adjustments especially when the number of parameters increase, it is not a good replacement for engineer's judgment in identifying the appropriate parameters for history matching and the weights 
assigned to the variables being matched. Based on what was mentioned, during last two decades there have been many efforts to improve this branch of science in a way that could be applicable in the real world. But despite all the attempts, due to increasing rate of complexity and resulotion in the reservoir models, there is a huge hesitation about the practicality and potential of these methods to handle highly complicated real reservoir models. This makes automated (assisted) history matching still a challenging research topic. However, with no doubt assisted history matching is an effective and fast tool to learn integrity of input data.

\subsection{Application of Neural Networks in petroleum engineering}

Artificial neural networks (ANNs), usually called Neural Networks (NNs), are algorithms that were originally motivated by the goal of having machines that can mimic the brain. They are non-digital, intensely parallel and distributive information processing systems that are the state of the art technique for many applications.

A neural network consists of an interconnected group of artificial neurons. NNs are physical cellular systems capable of obtaining, storing information, and using experiential knowledge. In fact, a NN is an adaptive system that adjusts its structure based on output and input information that flows through the network during the learning phase (Mohaghegh S. D., 1995). A neural network consists of an interconnected group of artificial neurons and its structure is an idea coming originally from human neural system.

Any typical neural network data processing procedure has two major steps: the learning and application step. At the first step, a training database is needed to train the networks. This dataset includes an input vector and a known output vector. Each one of the inputs and outputs are representing a node or neuron. In addition, there are one or more hidden layers. The objective of the learning phase is to adjust the weights of the connections between different layers or nodes. After setting up the learning samples, in an iterative approach a sample will be fed into the network and the resulting output will be compared with the known output. If the result and the known output are not equal, changing the weights of the connections will be continued until the difference is minimized. After acquiring the desired convergence for the networks in the learning process, the validation dataset is applied to the network for the validating step (Hagan, Demuth, \& Beale, 2002) (Haykin, 1998). Figure 5 depicts the input, hidden and output layers and their connections. 


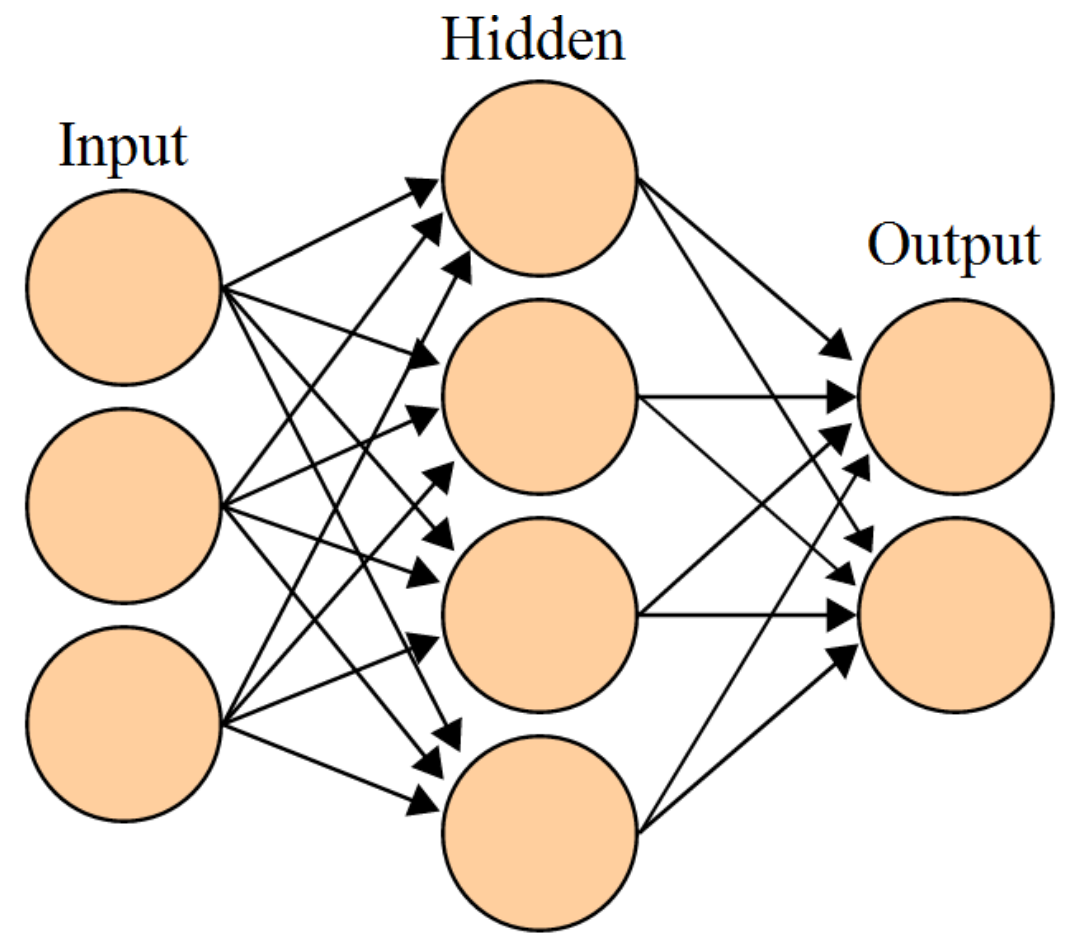

Figure 5- An artificial neural network is an interconnected group of nodes

Shahab Mohaghegh is one of the pioneers in applying Artificial Intelligence (AI) in petroleum engineering. He (Mohaghegh S. D., 1995) refers the main advantage of NN to the type of recognition ability and the difference in the mechanism with which human brain processes information compared to conventional digital computers. Computers are fast and accurate tools in performing prepared instructions. On the other hand, human brain performance is tremendously slower but more efficient than computers at computationally complicated jobs such as speech and other pattern- recognition problems.

NNs can be helpful tool to solve many conventional and unconventional problems in petroleum engineering. Although they have a long history, their popularity in petroleum engineering started three decades ago (Ali, 1994). Since this time, the applications of NNs in addressing conventional problems of petroleum industry have been widely studied. Following are a brief list of different applications of NNs in petroleum engineering:

- Application of NNs in well log interpretation (Baldwin, Otte, \& Whealtley, 1989) (Jong-Se \& Jungwhan, 2004) (Masoud, 1998)

- Using NNs in well test data analysis (Al-Kaabi \& Lee, 1990) (Ershaghi, Li, Hassibi, \& Shikari, 1993) (Athichanagorn \& Horne, 1995) (Sultanp \& Al-Kaabi, 2002) 
- NNs a helpful tool in reservoir characterization (Mohaghegh., Arefi, Ameri., \& Rose., 1995) (Ahmed, Link, Porter, Wideman, Himmer, \& Braun, 1997) (Singh, Painuly, Srivastava, Tiwary, \& Chandra, 2008)

- Application of NNs to calibrate seismic attributes (David, 1993), seismic pattern recognition (Yang \& Huang, 1991), inversion of seismic waveforms (Roth \& Tarantoia, 1992)

- Prediction of PVT data (Briones, Rojas, Moreno, \& Martinez, 1994) (Gharbi \& Elsharkawy, 1997) (Osman, Abdel-Wahhab, \& Al-Marhoun, 2001) (Oloso, Khoukhi, Abdulraheem, \& Elshafei, 2009)

- Identifying fractures and faults (L.Thomas \& Pointe, 1995) (Key, Nielsen, Signer, Sønneland, Waagbø, \& H. Veire, 1997) (Sadiq \& I.S. Nashawi, 2000) (Aminzadeh \& deGroot, 2005)

- Detecting hydrocarbons (Cheng-Dang, Wu, Mo, Zhu, \& Xu, 1994) (Aminzadeh \& deGroot, 2005), forecast formation damage (Nikravesh, Kovscek, Johnston, \& Patzek, 1996) (Kalam, Al-Alawi, \& Al-Mukheini, 1996) etc

\subsection{Artificial Intelligence (AI) assisted History Matching}

Reservoir simulation and modeling is utilized throughout field development in different capacities. Although in some cases they are not perfect, they are mostly the best realization of the subsurface. Sensitivity analysis, uncertainty assessment, history matching, production forecasts and operations optimization using reservoir simulators is conventional in full field model studies (Ertekin, Abou-Kassem, \& King, 2001). Realistic modeling of the complexities of a reservoir requires large number of grid blocks. As the complexity of a reservoir increases and consequently the number of grid blocks, so does the time required to accomplish the abovementioned tasks. Even though parallel processing features of some commercial simulators can take advantage of computational hardware advancements in order to perform many runs at the same time, it cannot still solve the problem properly. Today, finding a method to perform a real time analysis is a hot topic in academia and industry. It is obvious that with the available resources, numerical and conventional simulators cannot address the problem of convergence time for highly complicated reservoir models.

During the last decade, there has been lots of effort to find alternative methods to reduce the amount of CPU time needed to execute a numerical full field model. Artificial Intelligence (AI) methods are one of most famous and efficient examples of these kinds of techniques. 
Zangl et al (2006) trained a NN as a proxy model by using a limited number of simulation runs of a gas storage model. Then they applied this proxy model to replace the numerical model in order to make hundreds and thousands runs in a very short time in an optimization loop. One of the objectives was to perform history matching. The results were acceptable and had low computational expenses compared to using numerical reservoir simulation.

The objective of Rodriguez et al's (2007) work was to accelerate history match process by applying singular value decomposition method. This method helped them save $75 \%$ of total CPU time. At the same time, they used a NN in order to reduce number of simulations and help increase the accuracy of solution. Silva et al (2006) (2008) presented the application of global optimizers combined with NNs to address the history matching problem. Their results supported the potential of NNs to reduce the computational effort in history matching process. Sampaio et al (2009) used feed-forward neural networks as nonlinear proxies of reservoir simulation to speed up history matching. The focus of their work was to discuss the technical criteria that will lead to successful implementation of NN. The points they mentioned in this paper are very crucial for researchers who are interested in applying $\mathrm{NN}$ in petroleum engineering problems.

In this study, we are attempting to speed up the traditional history matching process. The focus of our work is on a relatively new technology known as Surrogate Reservoir Model (SRM). SRM has been introduced as a tool for addressing many time-consuming operations performed with reservoir simulation models (Mohaghegh S. D., 2006). In 2006, SRM was presented for the first time by Shahab Mohaghegh to solve problem of time-consuming runs for an uncertainty analysis of a giant oil field with 165 horizontal wells in the Middle East. The reservoir simulation model included about one million grid blocks and took 10 hours to run using a cluster of twelve $3.2 \mathrm{GHz}$ processors. In his study, SRM was used as an objective function for a Monte Carlo Simulation to build hundreds and thousands of simulation runs in a very short time compare to numerical simulator. Mohaghegh defines a Surrogate Reservoir Model, "SRMs are ensemble of multiple, interconnected neuro-fuzzy systems that are trained to adaptively learn the fluid flow behavior from a multi-well, multilayer reservoir simulation model, such that they can reproduce results similar to those of the reservoir simulation model (with high accuracy) in real-time.” (Mohaghegh S. D., Liu, Gaskari, Maysami, \& Olukoko, 2012b, p. 2). Since 2006, application of SRMs as an accurate and rapid replica of a numerical simulation model have been reviewed (Mohaghegh, Modavi, Hafez, \& Haajizadeh, 2009) (Mohaghegh S. , 2009) (Mohaghegh S. , 2010) (Mohaghegh S. D., Liu, Gaskari, Maysami, \& 
Olukoko, 2012b) (Mohaghegh, Amini, Gholami, Gaskari, \& Bromhal, 2012) (Amini, Mohaghegh, Gaskari, \& Bromhal, 2012). Mohaghegh in another paper (2011) exclusively explains all types of AI-based reservoir models, particularly surrogate reservoir models. 


\section{Chapter 2 Methodology}

\subsection{Methodology}

Mohaghegh (2011) indicates the source of SRMs in his recently published paper. These models are developed based on representative spatio-temporal databases. Building this database is the first step of developing AI-based reservoir models. The main objective of this database is to teach the AI model the whole process of fluid flow phenomena in the reservoir. There are different criteria to categorize the AI models. One classification factor is the source of these databases, which could be a base reservoir simulation model or from the field data. The database to build an SRM are feeding by a numerical reservoir simulation model, while if the source of database is actual field data then the AI-based model is called a Top-Down, Intelligent Reservoir Model, or Top-Down Model (TDM) for short (Mohaghegh S. D., 2011). In general, this database should meticulously provide static and dynamic information of the reservoir. The quality and quantity of this database determines degree of success in developing a successful AI-based reservoir model including an SRM (Mohaghegh S. D., 2011).

As mentioned earlier, history match techniques are used in reservoir modeling to fine-tune reservoir properties such as porosity and permeability by matching the measured production data (pressure or production profiles at each well). The objective of this study was to examine the utility of Surrogate Reservoir Model as a tool for assisted history matching. In this project, a SRM was created for a synthetic but highly heterogeneous and complex oil field, with 24 production wells and 30 years of production history. The aim of the project was to achieve a match for the production history by tuning the static data (permeability distribution). SRM was trained using several heterogeneity realizations and then validated by a blind simulation run. Finally, the full field model was substituted by the trained SRM for the history match process. It is worth mentioning that this project was just a proof of concept and we are trying to show the potential of SRM to assist in the history matching process. Further studies on a variety of $t$ cases should be considered in the future. 
Following are the steps involved in developing the SRM:

1. Model development and dataset generation:

a. SRMs are developed using a representative spatio-temporal database developed based on the reservoir simulation models. The extent that how good this database represents the fluid flow phenomena in the reservoir, is determining the degree of success in developing an SRM (Mohaghegh S. D., 2011). Because of important effects of this database on the developed SRM, high attention and care should be involved to develop the dataset. Therefore, this part is one of the most time consuming steps in an SRM development.

b. Development of a heterogeneous reservoir model using a commercial simulator (the model specifications will be explained in the following). A base-case permeability distribution was used to initiate the modeling process. In addition to the base model, ten different realizations with various permeability distributions were created to train NN. Another realization as a blind model was built to validate NN models as well.

c. In order to apply time series data, a tiering system was applied based on the Voronoi diagram.

d. Different static and dynamic data were extracted from the simulation models in order to build the spatio-temporal database.

2. SRM Development:

a. Key performance indicator (KPI) analysis was performed to identify and rank the influence of different reservoir characteristics on the reservoir performance.

b. An SRM was trained and calibrated using ten geological realizations of the reservoir.

c. The SRM was validated using a complete blind (eleventh) realization of the reservoir.

3. History Matching:

a. The SRM was used to calculate the oil production rates of 24 wells during 30 years (2000 to end of 2029). Consequently, oil production of all 24 wells was history matched in a short period of time by modifying the permeability distribution throughout the reservoir.

4. The final output of history matching is a permeability distribution that is compared with the original permeability distribution used as input to the full field model. 
The original (actual) permeability distribution was used for comparison purposes after the completion of the history matching process. Figure 6 describes different steps involved in the project.

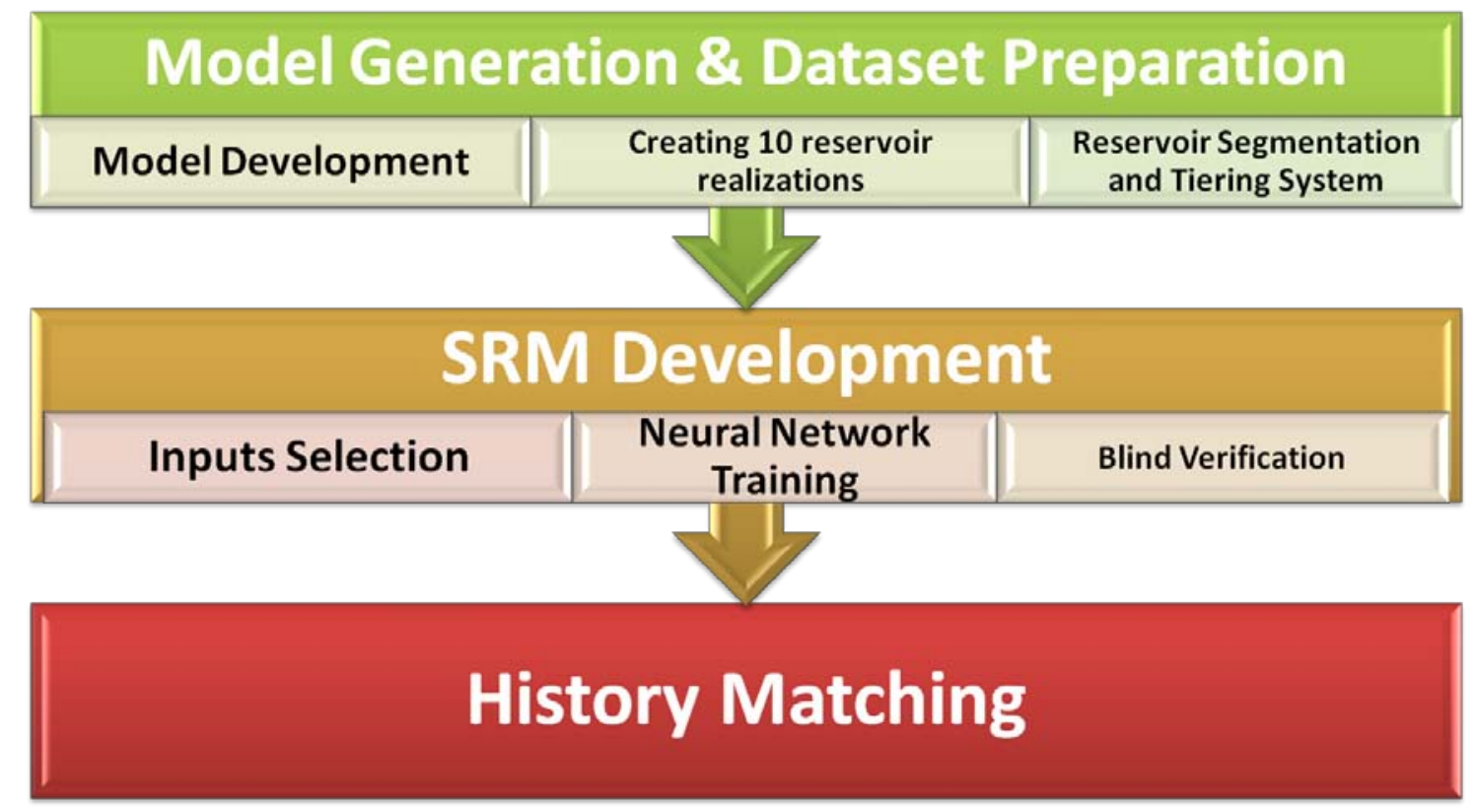

Figure 6- A diagram describing different steps in the project

\subsection{Model Generation and Dataset Preparation}

\subsubsection{Base Model Description and Historical Data}

The base simulation model is a single porosity oil reservoir, which was constructed in CMG$\mathrm{IMEX}^{\mathrm{TM} 1}$. The reservoir has been divided into 4800 Non-Orthogonal grid blocks, 80 in $\mathrm{X}$ direction and 60 in $\mathrm{Y}$ direction. The reservoir is single layer and thickness is variable in different girds.

The field is producing oil at initial pressure of 2000 psi and bubble point pressure of 300 psi, therefore it is expected the candidate reservoir will be producing oil for a long time in an under-saturated condition. The model is synthetic and does not represent a real field. The model includes 24 vertical wells distributed in the reservoir. Figure 7 shows a 3D and 2D view of the reservoir structure. Figure 8 and Figure 9 show 3D and 2D views of porosity and grid thickness distributions.

\footnotetext{
${ }^{1}$ Computer Modelling Group
} 

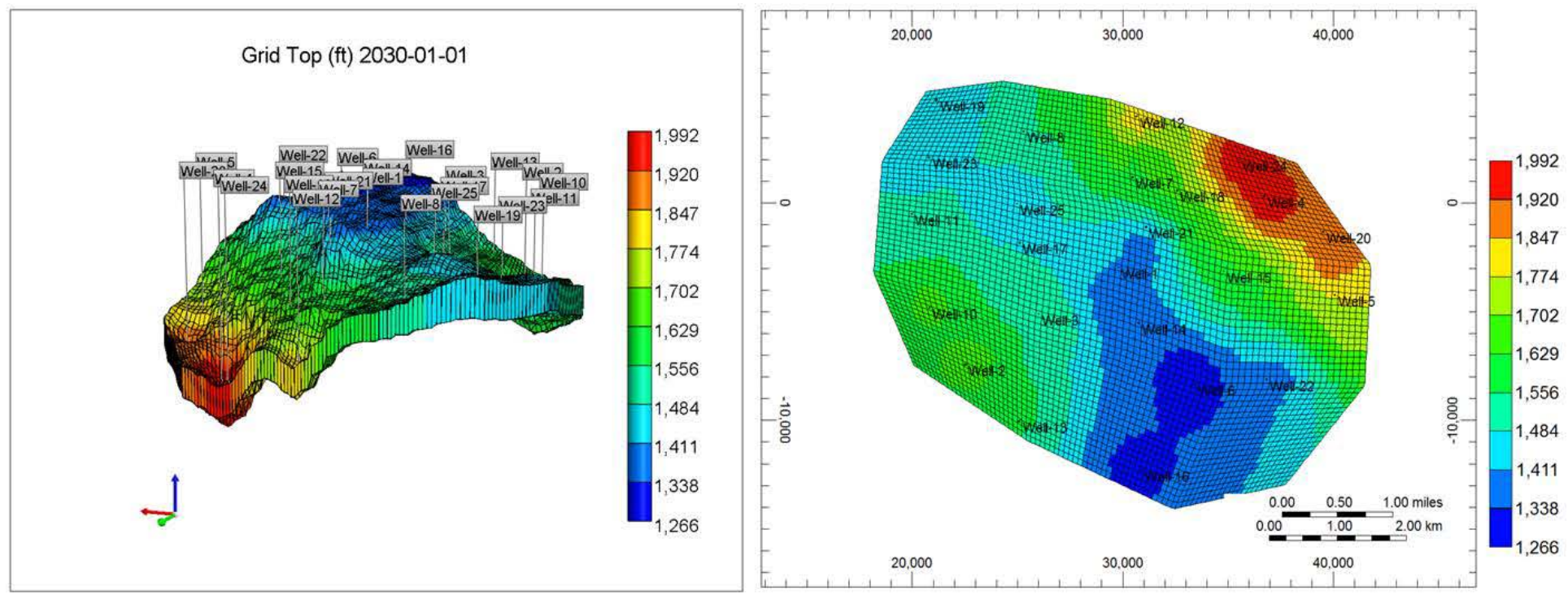

Figure 7- 3D and 2D top views of simulation model

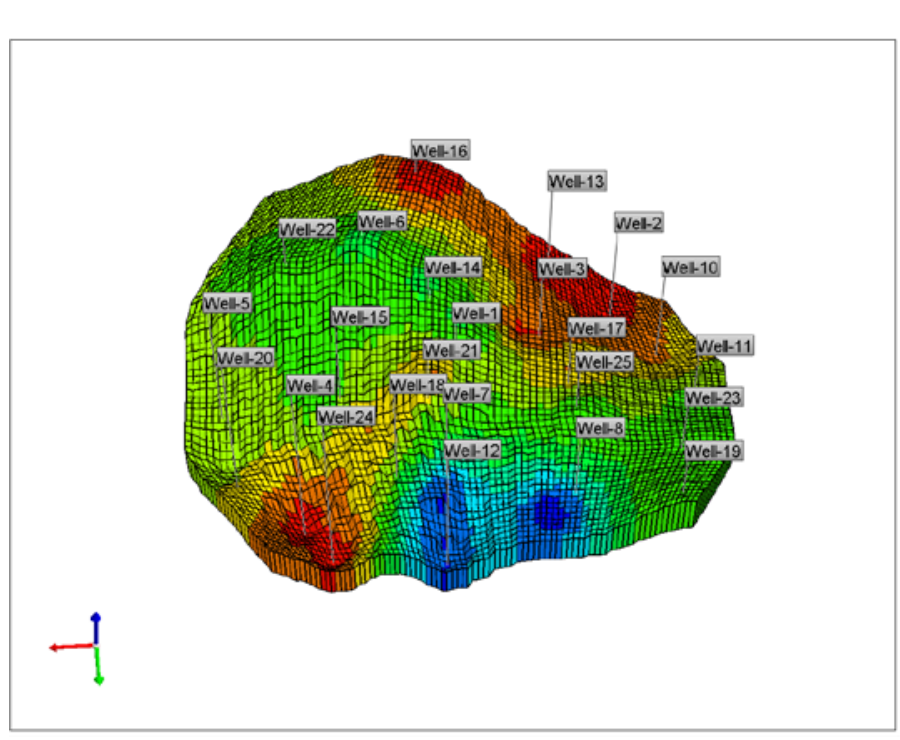

\section{Porosity}

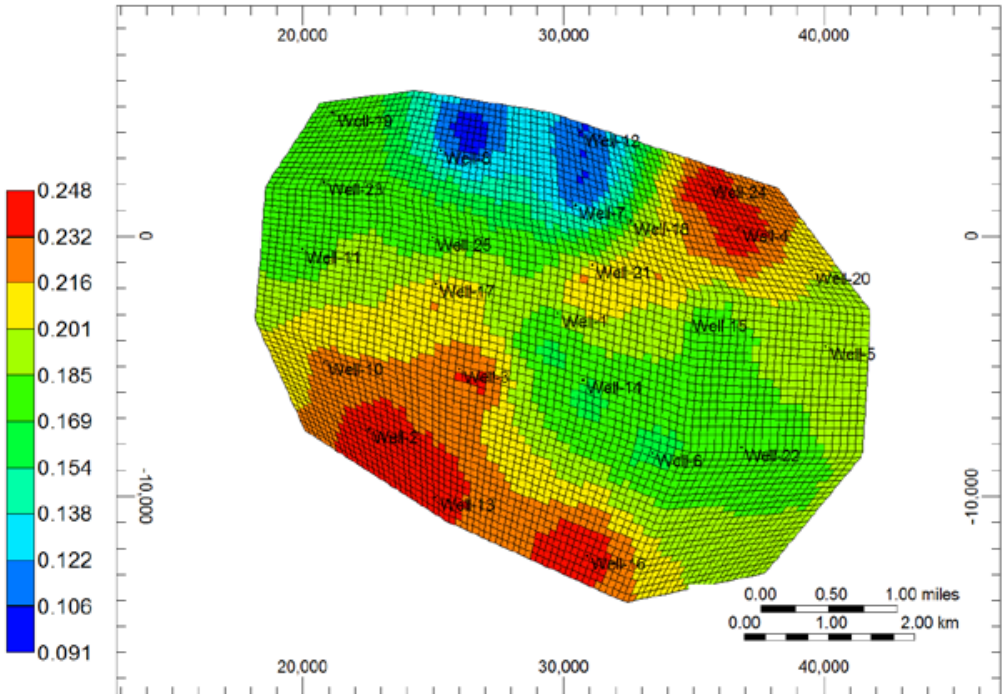

Figure 8- 3D and 2D views of porosity distributions 


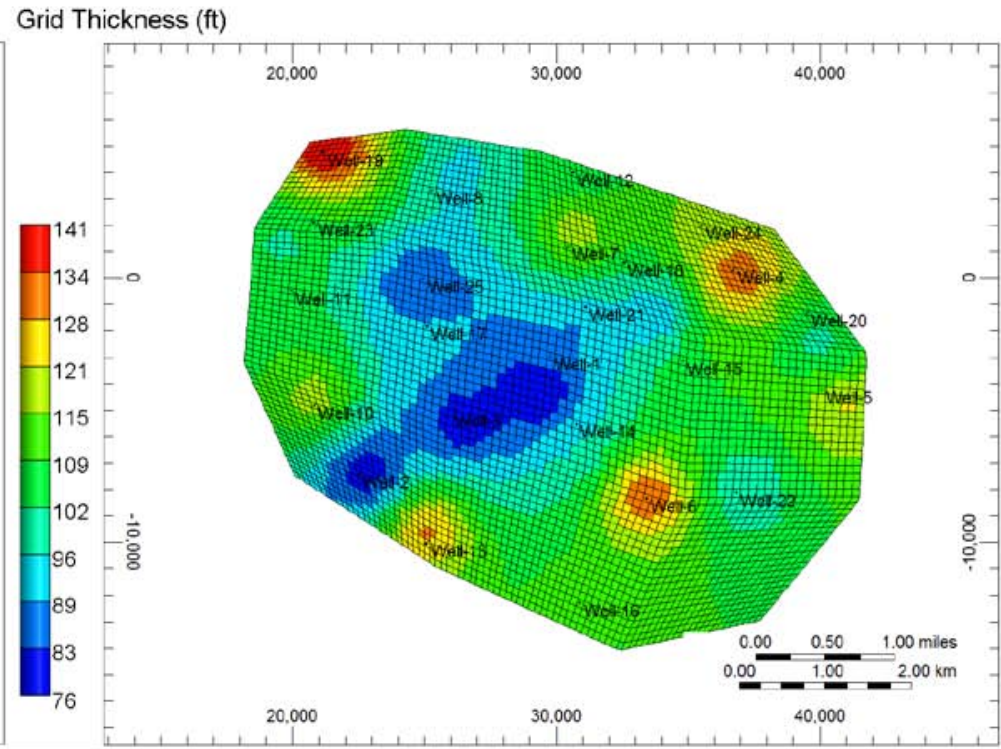

Figure 9- 2D and 3D views of Thickness map

The given permeability range for the base model is 10 to $75 \mathrm{md}$ (Figure 10). In addition, the geological information from the field indentifies a high permeable zone (Figure 11).

Permeability I (md) 2000-01-01

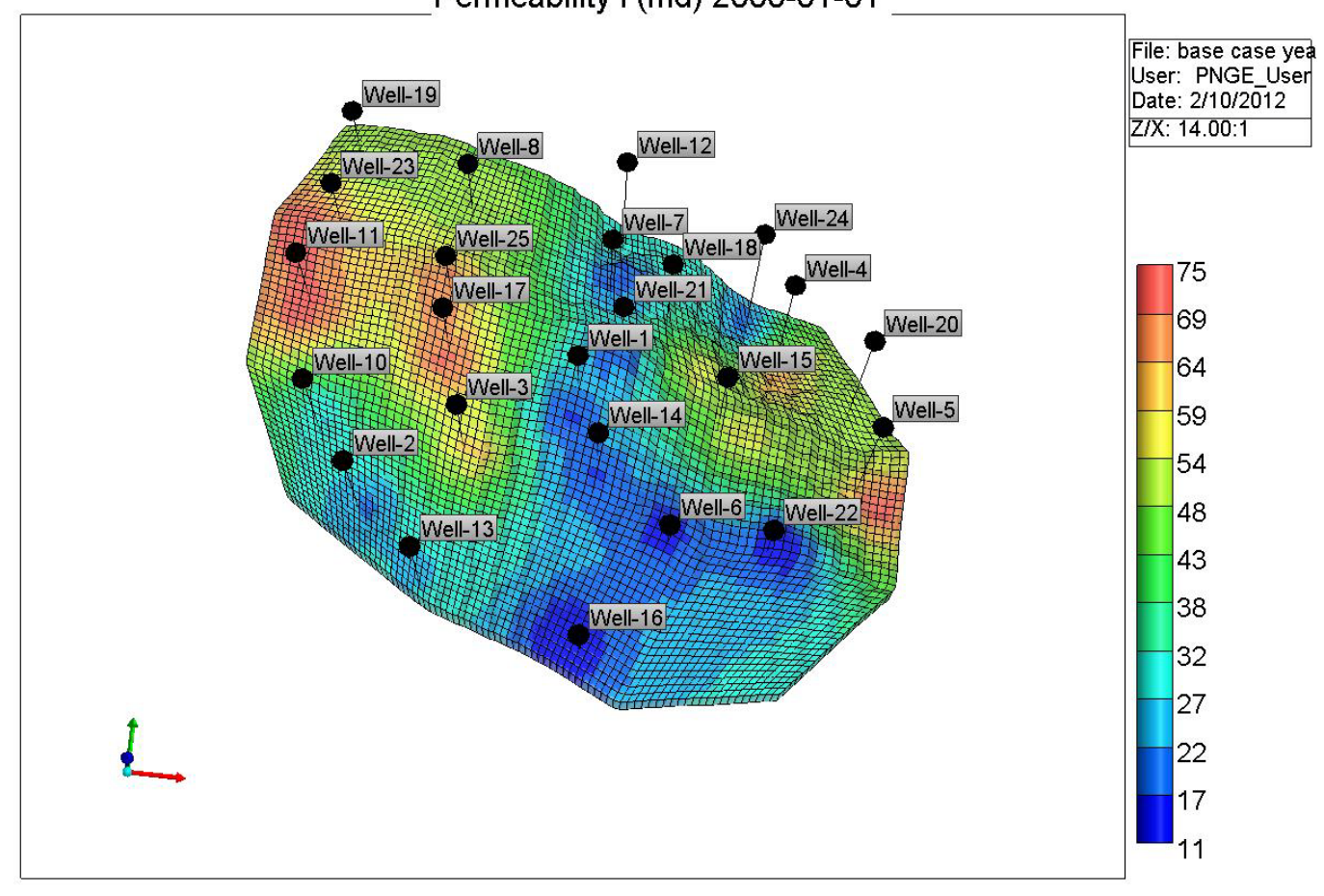

Figure 10- Given Permeability map for the base case 


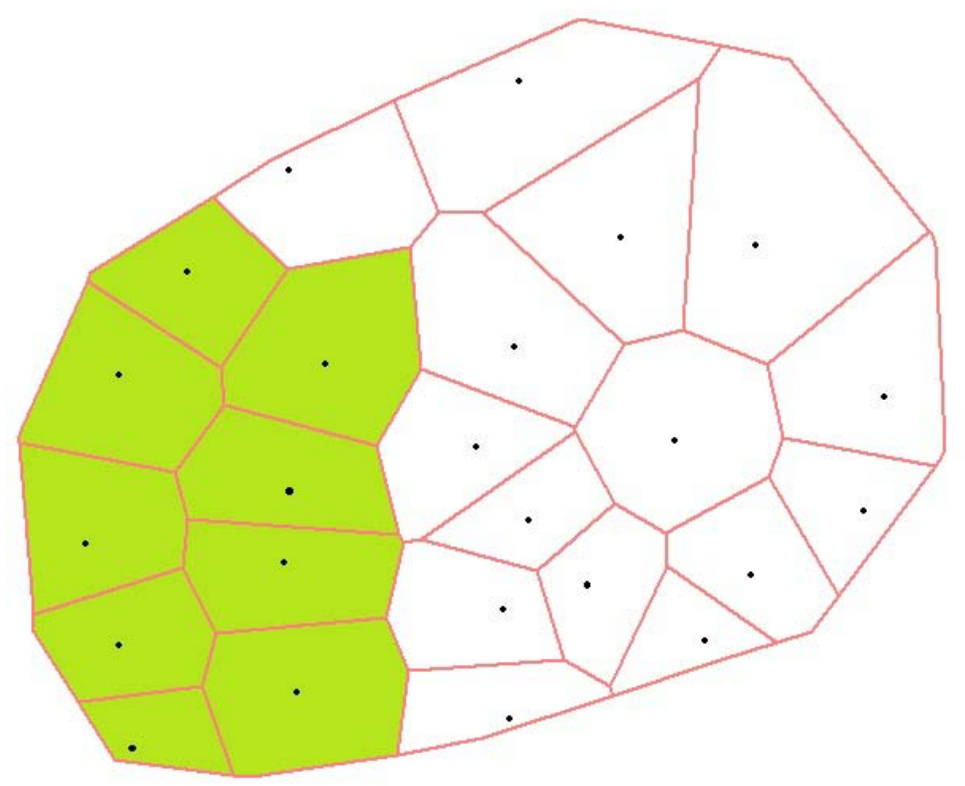

Figure 11- Geological information identifies a high permeable zone in the field

Twenty-four production wells have been drilled in the field and they produce oil for 30 years. The production constraint is minimum bottom-hole pressure (BHP). Figure 12 demonstrates the recorded monthly bottom-hole pressure for a couple of wells. Other wells' BHP are available in Appendix A. Table 2 contains the values of porosity, thickness and permeability in the base model at wells' locations.

Well Constraints- Monthly BHP (psi)

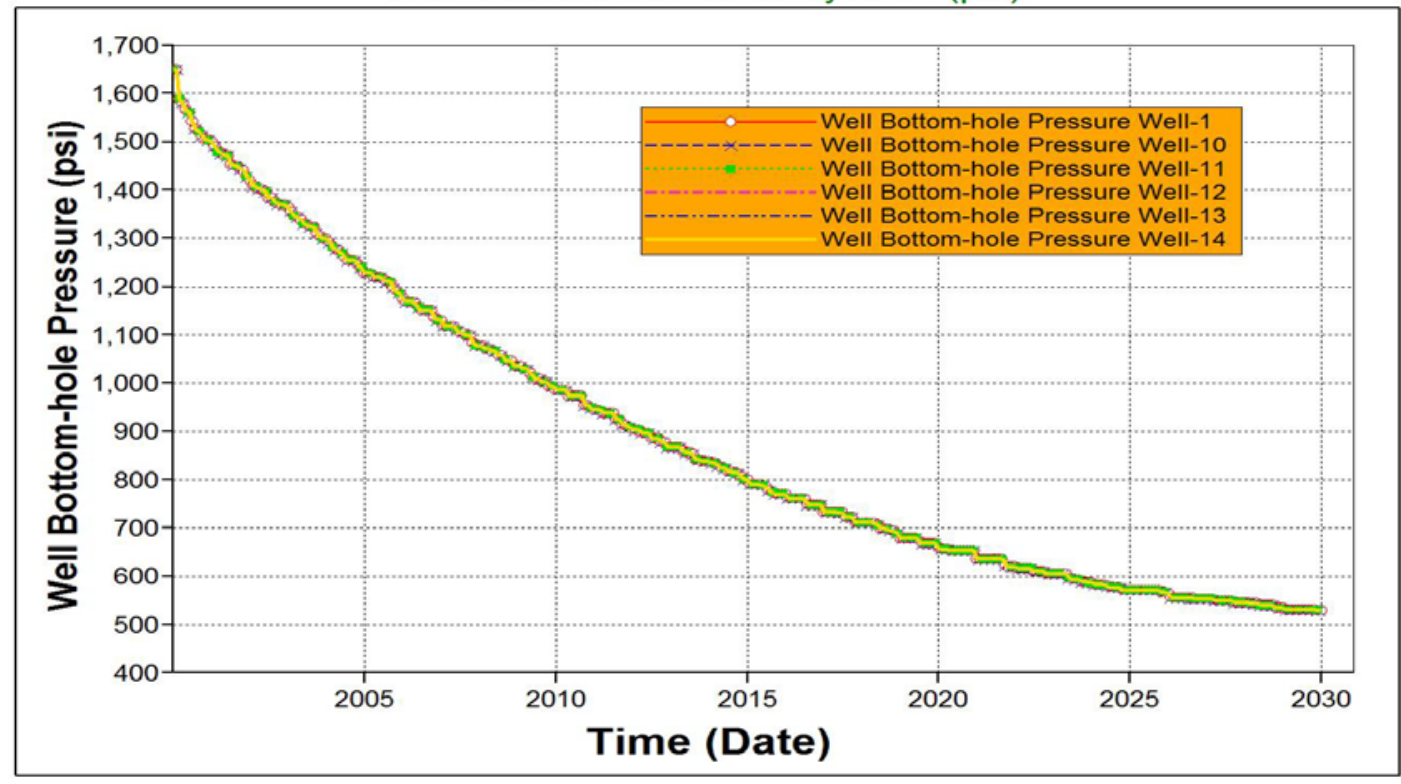

Figure 12- Bottom-hole pressure as constraints for the production wells 
Table 2- Porosity, Thickness and Permeability values at the Wells Locations

\begin{tabular}{|c|c|c|c|c|c|}
\hline Well & $\mathbf{x}$ & $\mathbf{Y}$ & Porosity, $\%$ & Thickness, ft & Base Case- Perm, md \\
\hline Well-1 & 29797.76107 & -2969.596922 & $18.5 \%$ & 85.0 & 25 \\
\hline Well-10 & 20818.3326 & -4826.669843 & $21.9 \%$ & 116.6 & 28 \\
\hline Well-11 & 19984.88967 & -508.592905 & $18.1 \%$ & 105.5 & 72 \\
\hline Well-12 & 30663.40551 & 3985.05564 & $10.6 \%$ & 104.9 & 30 \\
\hline Well-13 & 25095.62265 & -10051.09466 & $24.2 \%$ & 128.0 & 27 \\
\hline Well-14 & 30772.75466 & -5525.997855 & $17.1 \%$ & 89.0 & 19 \\
\hline Well-15 & 34811.17672 & -3138.275779 & $18.5 \%$ & 104.7 & 52 \\
\hline Well-16 & 30905.66899 & -12314.17555 & $24.5 \%$ & 110.3 & 12 \\
\hline Well-17 & 25120.29011 & -1838.947422 & $20.8 \%$ & 89.6 & 67 \\
\hline Well-18 & 32589.76022 & 559.516981 & $17.4 \%$ & 107.1 & 33 \\
\hline Well-19 & 21168.47142 & 4729.837924 & $18.0 \%$ & 138.0 & 51 \\
\hline Well-2 & 22532.65691 & -7445.417454 & $24.3 \%$ & 80.2 & 22 \\
\hline Well-20 & 39543.65566 & -1323.20883 & $20.2 \%$ & 109.7 & 50 \\
\hline Well-21 & 31120.44172 & -1109.803689 & $20.2 \%$ & 94.0 & 24 \\
\hline Well-22 & 36845.38866 & -8125.316388 & $17.2 \%$ & 97.3 & 14 \\
\hline Well-23 & 20810.84596 & 2081.715526 & $18.0 \%$ & 105.1 & 66 \\
\hline Well-24 & 35542.90925 & 1968.335089 & $24.4 \%$ & 118.5 & 22 \\
\hline Well-25 & 24985.5662 & -33.008043 & $18.2 \%$ & 82.7 & 68 \\
\hline Well-3 & 26008.87163 & -5113.980521 & $23.0 \%$ & 82.2 & 59 \\
\hline Well-4 & 36734.4498 & 302.654284 & $24.4 \%$ & 133.5 & 63 \\
\hline Well-5 & 40082.1137 & -4241.561545 & $19.3 \%$ & 113.0 & 61 \\
\hline Well-6 & 33448.56813 & -8339.499421 & $16.3 \%$ & 133.0 & 14 \\
\hline Well-7 & 30483.26314 & 1187.889606 & $12.9 \%$ & 116.2 & 22 \\
\hline Well-8 & 25311.7145 & 3298.650452 & $12.3 \%$ & 96.5 & 50 \\
\hline
\end{tabular}

The wells produce for 30 years, starting in 2000/01/01. The available historical data include oil rate production and cumulative oil production for all the wells. Figure 13 shows the oil rate and cumulative oil for four wells. The complete historical data are given in Appendix A.

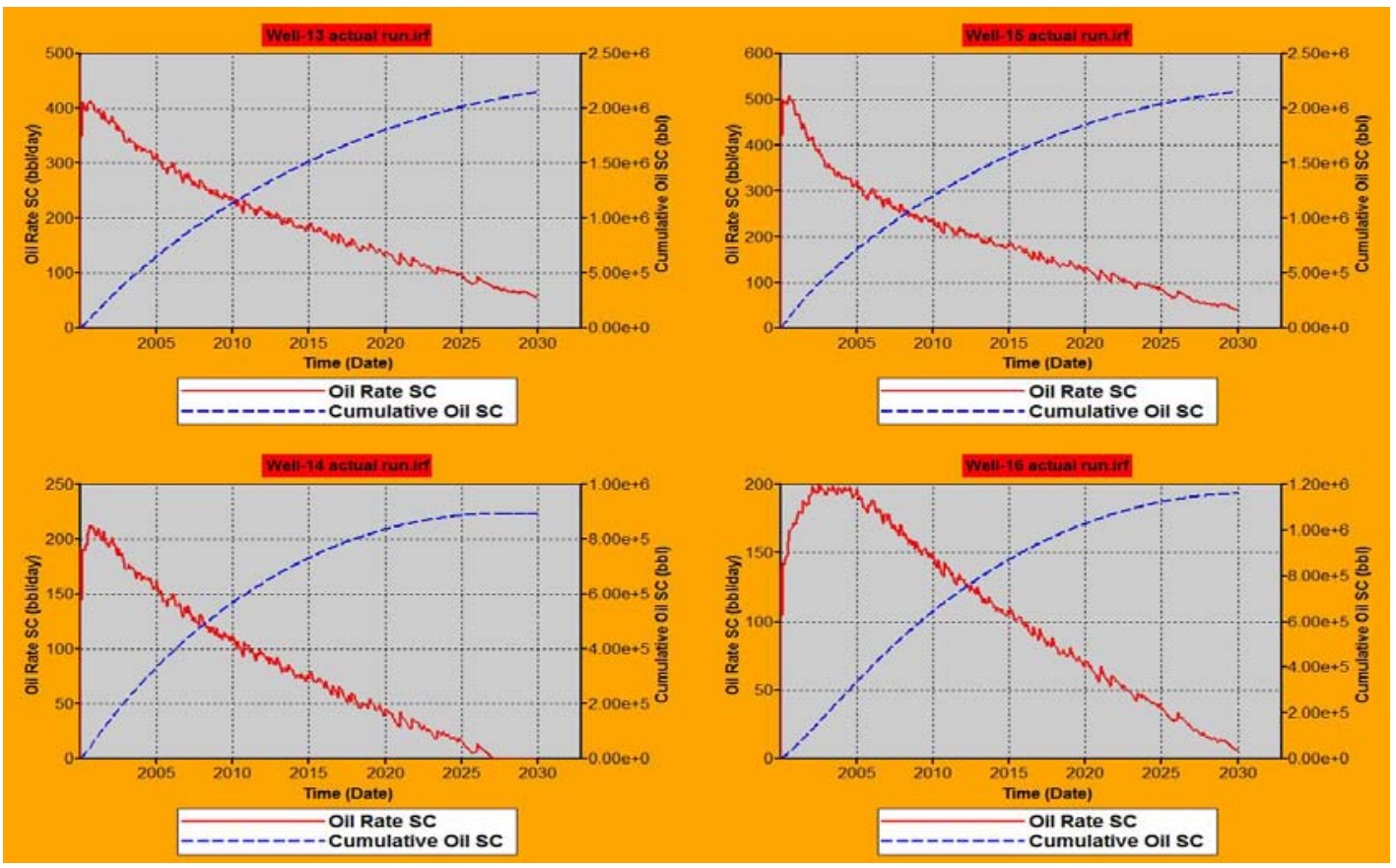

Figure 13- Production Historical data for some wells, rest of wells are available in Appendix A 


\subsubsection{Training Realizations}

In order to introduce the uncertainties involved in the reservoir simulation model to the SRM, a small number of simulation runs should be made. This approach is common in SRM and the response surface methods. However, there is a main difference between these two methods, which is the functional forms behind these models. Response surface and other reduced models are developed using statistical approaches, which use predetermined functional forms. Then the output of reservoir simulation models are fitted to these predetermined functional forms. Mostly to match these functional forms, hundreds of runs are needed. On the other hand, the mechanism of SRM is not based on the predetermined functional forms. Mohaghegh (2012a) addresses this mechanism as, "SRMs are developed using universal function approximation technology that will adapt and fit an infinite set of functional forms that may change from one set to another many times within the time and space domain.”

The above introduction explains why it is possible to develop an SRM just by couple of runs. However, there is no algorithm to find the optimum number of simulation runs to build an SRM. The best number to train the SRM is mostly rule of thumbs. It changes depending on the intricacy and heterogeneity of the reservoir model. Nevertheless, it is obvious if the number of simulation runs is small, the SRM will not be able to reproduce the simulator results properly. Otherwise, if the number of simulation runs is too big, there is no reason to develop an SRM since the solution is close to the original problem, which is the high number of simulation runs.

In this study, ten different realizations of the base model were designed to develop the SRM. By utilizing the provided permeability distribution map, ten different permeability maps were created. The range of permeability for the base model is 10 to $70 \mathrm{md}$. Due to the uncertainty involved in reservoir properties, we considered a range of 10 to $200 \mathrm{md}$ to create the permeability distributions. Developing the permeability map was based on assigning different values at the wells' locations and using a geo-statistical method "Inverse Distance Method" provided by Builder ${ }^{\mathrm{TM} 1}$ (CMG). Subsequently, to create ten different cases of permeability values at well location, one experimental method (Latin Hypercube) was used. Table 3 summarizes generated permeability values at the wells' locations. The permeability values for each well have been ranged by color, in which red and blue represent minimum and maximum value respectively.

\footnotetext{
${ }^{1}$ Computer Modelling Group
} 
Table 3- Different permeability values at wells' locations for creating permeability maps

\begin{tabular}{|c|c|c|c|c|c|c|c|c|c|c|}
\hline \multirow{2}{*}{ Well } & \multicolumn{3}{|c|}{ Permeabilty @ Wells' Location for 10 Runs applied in Training part } \\
\cline { 2 - 13 } & Run 1 & Run 2 & Run 3 & Run 4 & Run 5 & Run 6 & Run 7 & Run 8 & Run 9 & Run 10 \\
\hline Well-1 & 44.6 & 21.6 & 21.0 & 38.7 & 60.5 & 67.1 & 31.3 & 66.4 & 44.2 & 89.7 \\
\hline Well-10 & 40.3 & 29.8 & 51.5 & 140.8 & 49.7 & 142.8 & 146.9 & 87.5 & 113.6 & 107.3 \\
\hline Well-11 & 41.7 & 85.8 & 32.6 & 83.8 & 138.6 & 39.0 & 122.6 & 117.7 & 97.1 & 33.0 \\
\hline Well-12 & 33.4 & 18.0 & 44.7 & 51.9 & 40.1 & 21.2 & 89.8 & 73.9 & 75.5 & 34.9 \\
\hline Well-13 & 64.3 & 41.3 & 64.3 & 61.0 & 74.9 & 69.2 & 54.7 & 60.9 & 118.4 & 104.9 \\
\hline Well-14 & 17.6 & 42.8 & 30.0 & 31.0 & 38.1 & 28.4 & 43.1 & 56.1 & 35.2 & 62.9 \\
\hline Well-15 & 18.9 & 44.9 & 37.5 & 47.4 & 55.8 & 32.7 & 92.0 & 93.1 & 91.2 & 59.6 \\
\hline Well-16 & 21.7 & 40.0 & 29.9 & 32.5 & 41.1 & 33.9 & 43.0 & 57.8 & 38.8 & 66.8 \\
\hline Well-17 & 78.1 & 45.0 & 27.7 & 44.6 & 84.1 & 102.4 & 93.8 & 143.9 & 158.9 & 152.5 \\
\hline Well-18 & 34.6 & 24.6 & 40.2 & 56.8 & 29.5 & 44.1 & 75.7 & 74.9 & 54.6 & 81.3 \\
\hline Well-19 & 76.8 & 60.3 & 95.6 & 38.9 & 54.0 & 81.7 & 107.5 & 172.6 & 49.3 & 61.4 \\
\hline Well-2 & 58.8 & 42.9 & 52.6 & 92.2 & 78.0 & 105.4 & 93.7 & 81.6 & 107.2 & 119.5 \\
\hline Well-20 & 41.2 & 27.3 & 14.4 & 53.9 & 70.7 & 27.4 & 18.9 & 37.4 & 88.0 & 21.9 \\
\hline Well-21 & 19.5 & 22.4 & 39.5 & 27.4 & 19.6 & 47.5 & 50.5 & 63.4 & 60.7 & 89.6 \\
\hline Well-22 & 30.0 & 36.0 & 31.9 & 42.4 & 46.1 & 43.3 & 63.6 & 61.3 & 70.1 & 57.4 \\
\hline Well-23 & 58.6 & 67.8 & 60.4 & 117.6 & 109.0 & 59.6 & 82.4 & 55.8 & 47.6 & 73.2 \\
\hline Well-24 & 29.2 & 31.6 & 21.5 & 44.0 & 28.2 & 57.5 & 17.5 & 77.4 & 69.4 & 27.8 \\
\hline Well-25 & 29.5 & 88.8 & 46.1 & 68.4 & 31.7 & 109.8 & 159.9 & 112.4 & 182.8 & 153.0 \\
\hline Well-3 & 67.0 & 38.5 & 76.5 & 55.4 & 72.5 & 53.2 & 36.8 & 43.1 & 123.5 & 94.0 \\
\hline Well-4 & 26.5 & 37.5 & 32.9 & 71.0 & 66.7 & 42.0 & 31.6 & 29.8 & 22.8 & 19.0 \\
\hline Well-5 & 43.2 & 30.2 & 31.7 & 15.9 & 26.4 & 66.3 & 60.4 & 86.2 & 83.2 & 72.5 \\
\hline Well-6 & 24.5 & 38.1 & 34.9 & 40.3 & 46.5 & 38.5 & 62.3 & 63.4 & 56.7 & 61.7 \\
\hline Well-7 & 41.6 & 15.9 & 30.7 & 65.1 & 39.2 & 20.6 & 44.5 & 49.6 & 50.4 & 57.0 \\
\hline Well-8 & 54.1 & 79.0 & 73.4 & 112.9 & 101.4 & 71.8 & 193.9 & 161.8 & 140.1 & 131.8 \\
\hline
\end{tabular}

Figure 14 shows three different permeability maps; the rest of the realizations are listed in Appendix B. In these realizations, permeability distributions as well as the range of permeability values are different.

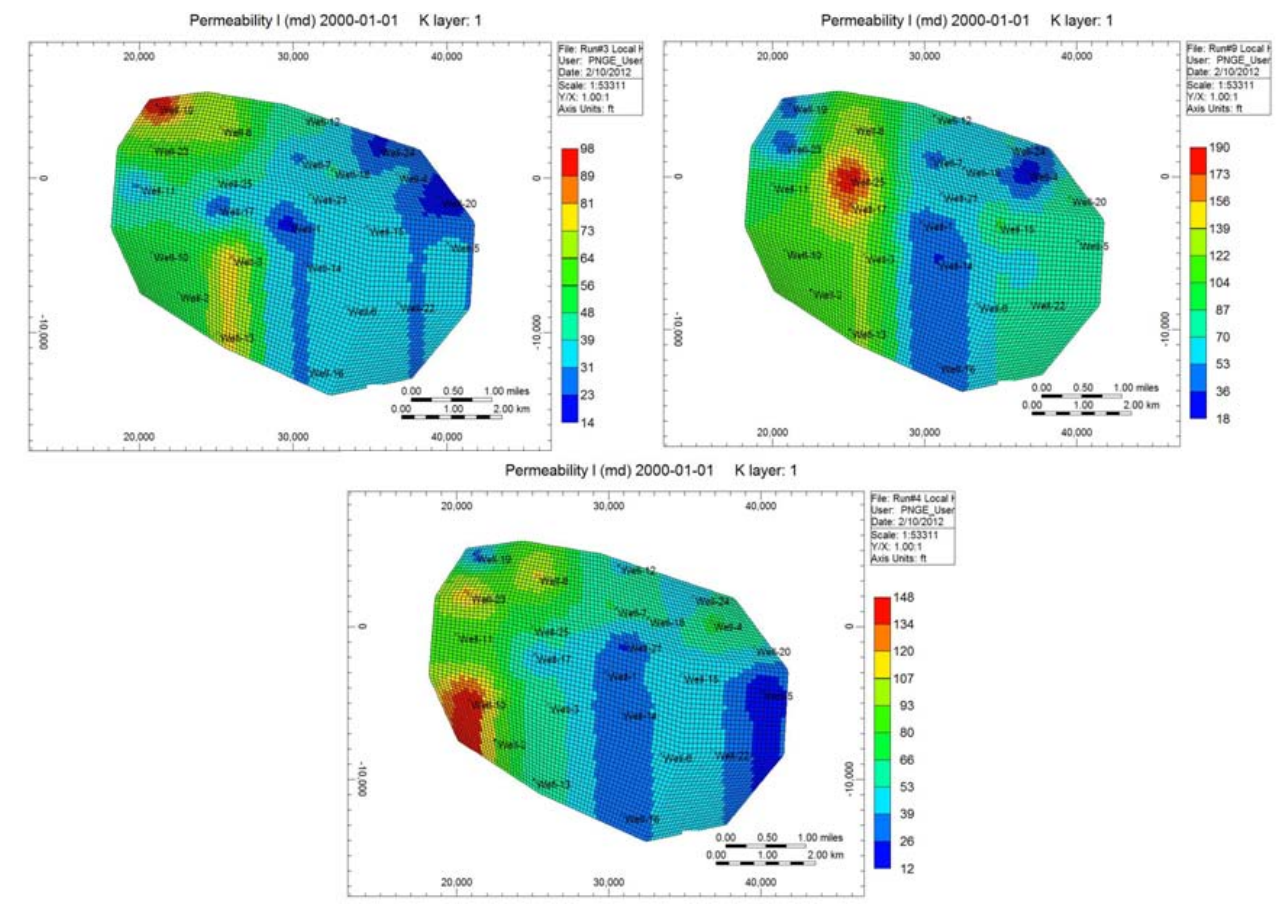

Figure 14- Different permeabilty distributions created to train SRM (the rest is available in Appendix B) 


\subsubsection{Reservoir Segmentation- Tiering System and offset wells}

In order to include the static data of adjacent grid blocks of a well in the spatio-temporal database, a tiering system was generated. Another objective of this part is to summarize the information of different grid blocks based on their influences on the wells' production. Different grid blocks have different impacts on the fluid flow depending on their distance from the production well. For example, the well grid block has the maximum influence on the production of a well, whereas the farther grid blocks have less impact on the production of a well. Therefore, this influence should be considered during the development of the database. In addition, the impacts of the neighborhood wells' production were considered (offset wells' effects).

The first step in identifying tiers is dividing the reservoir using the Voronoi algorithm. Based on this method the reservoir is assumed as a homogenous reservoir, and the drainage area for each well is calculated based on the Voronoi diagram. Let us assume an ideal and homogenous reservoir, in which all the wells produce at the same rate and at the same time. For this example, after a specific time the drainage area for each well will become a polygon, in which the boundaries have equal distance to the nearby wells. In other words, the polygons are separated using image wells theory, which says that a reservoir being produced under homogenous conditions and the same rate will result in a no flow boundary at the midpoint between two wells. In this study, the reservoir was divided into the Voronoi segments using IDEA $^{\text {TM } 1}$ (Figure 15). IDEA is a software application made by ISI $^{2}$ for the development of data driven, intelligent models. We will explain more about the applications of this software later in this chapter.

After creating the segments, in order to take the effects of different grid blocks into account, each drainage area is divided into four tiers. The first tier is the grid block that the well is located on. The second, third and fourth tiers stand for the outer surrounding grid blocks of each drainage area. Figure 16 shows different tiers for a drainage area. It should be noted that the number of grids in the first (1), second (8) and third (16) tiers are constant. Consequently, the last or fourth tier includes the rest of grid blocks.

\footnotetext{
${ }^{1}$ Intelligent Data Evaluation \& Analysis

${ }^{2}$ Intelligent Solutions Inc
} 


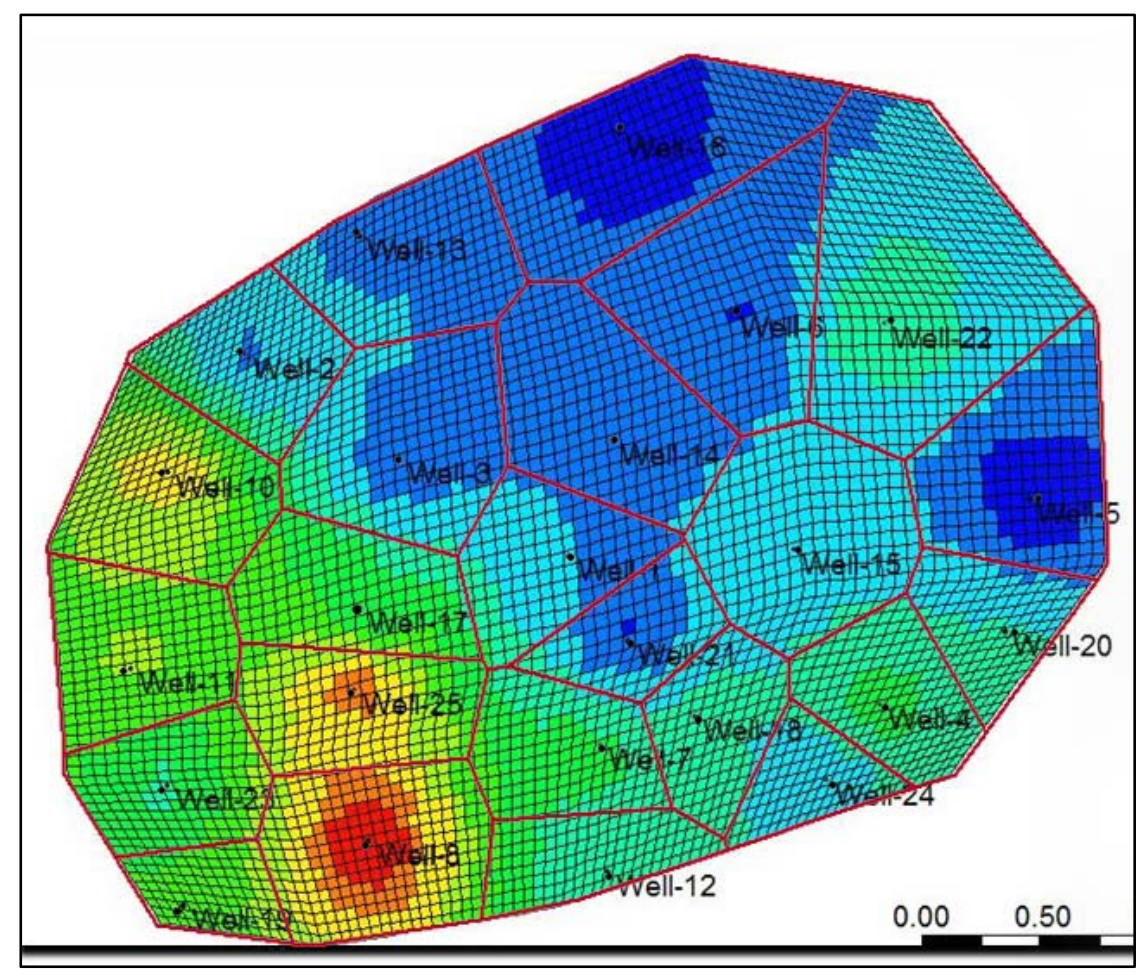

Figure 15- The reservoir divided to different drainage areas following Voronoi diagram

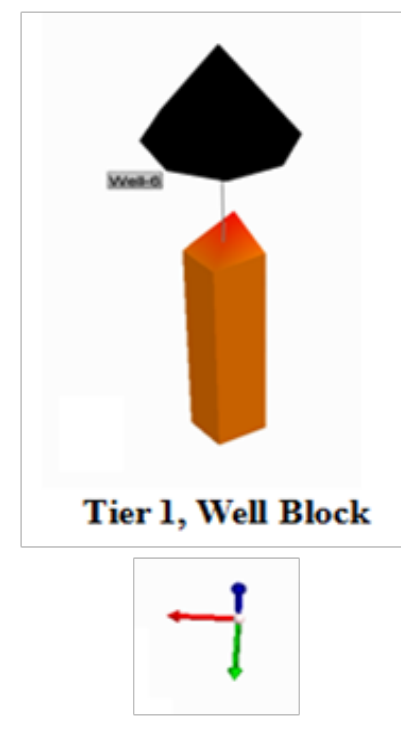

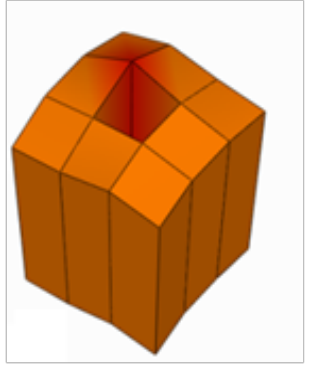

Tier 2

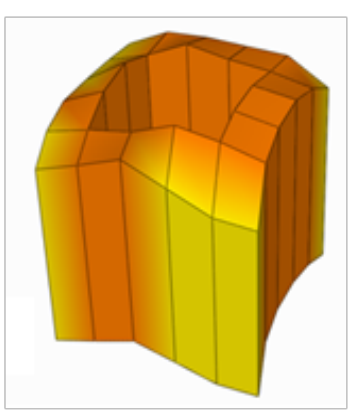

Tier 3

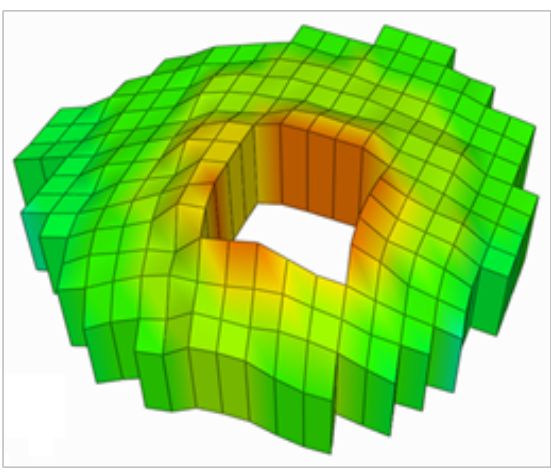

Tier 4

Figure 16- Different tiers were designed based on the Voronoi diagram

In order to consider the effect of nearby wells on the performance of a specific well, the three closest wells have been assigned as the offset wells. Generally, there are a couple of facts that affect the number of offset wells. The first one is the conductivity of a reservoir, which is a function of reservoir characteristics, particularly permeability. The more conduct a reservoir, the easier it is for the fluid to flow and see the other parts of a reservoir faster. Therefore, the number of offset wells will be higher in a conduct reservoir than in a tight reservoir. The 
second fact is the background of the reservoir. In a brown (mature) field with a long period of production, the reservoir has reached a further distance than a green field, which still is at the initial levels of production. Finally, the measureable impact (practical observation) of other wells' performances should be considered. Based on the diffusivity equation, the effect of a change in a part of system can be observed simultaneously all over the system. However, the amount of this change that is desired to be measured, is another factor.

In this study based on the reservoir characteristics of the reservoir, which is mainly a conductive reservoir, the three closest wells were considered as the offset wells. Since the process of choosing the number of offset wells is mostly a trial and error procedure, the stage is still open for arguing another number of offset wells. In addition, it is worth mentioning that the dimension of the spatio-temporal database should be unique, therefore a single number of offset wells was decided.

\subsection{SRM Development}

\subsubsection{Input Selection}

Up to this point, the required simulation runs have been designed, the runs were made, the reservoir was divided into the desired segments and three offset wells were recognized for each well. At this step, the inputs to develop the spatio-temporal database are selected. As it was mentioned, building this database is the most important step of developing an SRM. In the building of this database, the objective of the reservoir modeling should be considered (Amini, Mohaghegh, Gaskari, \& Bromhal, 2012) (Mohaghegh S. D., Liu, Gaskari, Maysami, \& Olukoko, 2012a). For instance, the objective of this study is to use the SRM to estimate the well parameters such as oil production. Therefore, the reservoir properties, which are affecting the objective of the study, have higher degrees of importance.

The database includes different types of data such as static and dynamic reservoir characteristics, operational constraints, etc. Static data refers to properties of the reservoir that are not changing through time, such as permeability, porosity, top and thickness. On the other hand, the dynamic data addresses variable parameters that are changing through time, such as oil production rate, bottom-hole pressure, and time.

The reservoir tiers and segmentation were explained in the previous section. One objective of the reservoir segmentation is to summarize the grids' properties based on the degree of influence for each grid block. For example, the well block is the most important grid block 
affecting the production; therefore, this block is considered a separate tier (tier1). However, for other tiers there is more than one grid block at each tier. Consequently, in order to assign one value to each tier, arithmetic mean calculation is performed. This calculation is done just for static properties.

Figure 17 summarizes the different types of data in the spatio-temporal database. The number of rows in this database is 24(number of wells) X 30 (number of production time steps per well). The number of columns is 92 (static and dynamic properties).

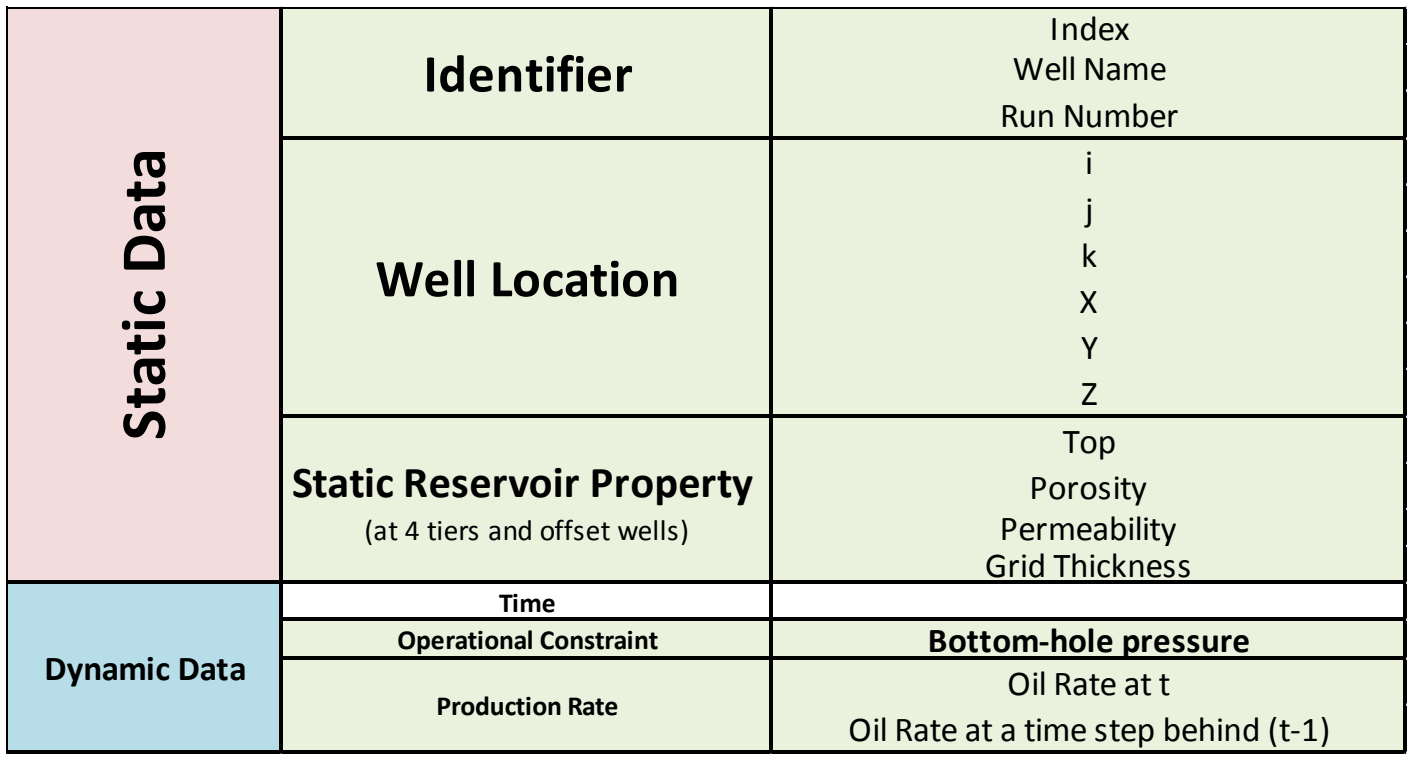

Figure 17- Different types of data in the spatio-temporal database

\subsubsection{Creating NN models}

After preparing the database, it is time to create the NN models. $\mathrm{N}$ order to generate $\mathrm{NN}$, software developed by Intelligent Solutions Inc (ISI) called IDEA ${ }^{\mathrm{TM}}$ is used (Intelligent Solutions Inc., 2012). IDEA provides multiple training paradigms for NN models including Generalized Regression (GR) and Back Propagation. Back Propagation, the most common training method to train the NN, is utilized to train the NN models. The time-based model development feature of this paradigm is appropriate for our study.

An SRM, depending on the application, could consist of one or multiple NN models. In this study, there are two separate NN models. The differences between these NNs are in inputs and output selections. The output of the first NN, called the Initialization model, is the oil rate at the end of the first year of production (2000/31/01). While the output of the second NN, named the Main model, is the oil rate for the other years of production. In addition, the inputs to train these models are different. 
The reason for making two different models goes back to the initial reservoir response to the production. In the early stages of production, reservoirs are at the initialization process and still at the unstable phase. Mostly at this level, the production of the target well has not been influenced by the nearby wells, and reservoir properties (static data) are the most influential parameters to characterize the production. Eventually the SRM would be the result of the combination of these two networks. The process of building the networks are the same for these models and will be explained gradually in the following sections.

\subsubsection{Importing Data Set}

This section and the following sections (2.3.3 to 2.3.5) mostly explain how to work with IDEA in order to make the NNs. The first step in building a NN is to import the prepared database into the software. After defining the project, IDEA provides an interface to import the database and define the type of data in the database (Figure 18). By default, all parameters have been defined as an "Input" but the user is able to label them as another type of data such as “Output”, “ID” (Identifier) or "Not Used”. At least one output must be chosen for each model. In this project, the oil rate is the output of the system.

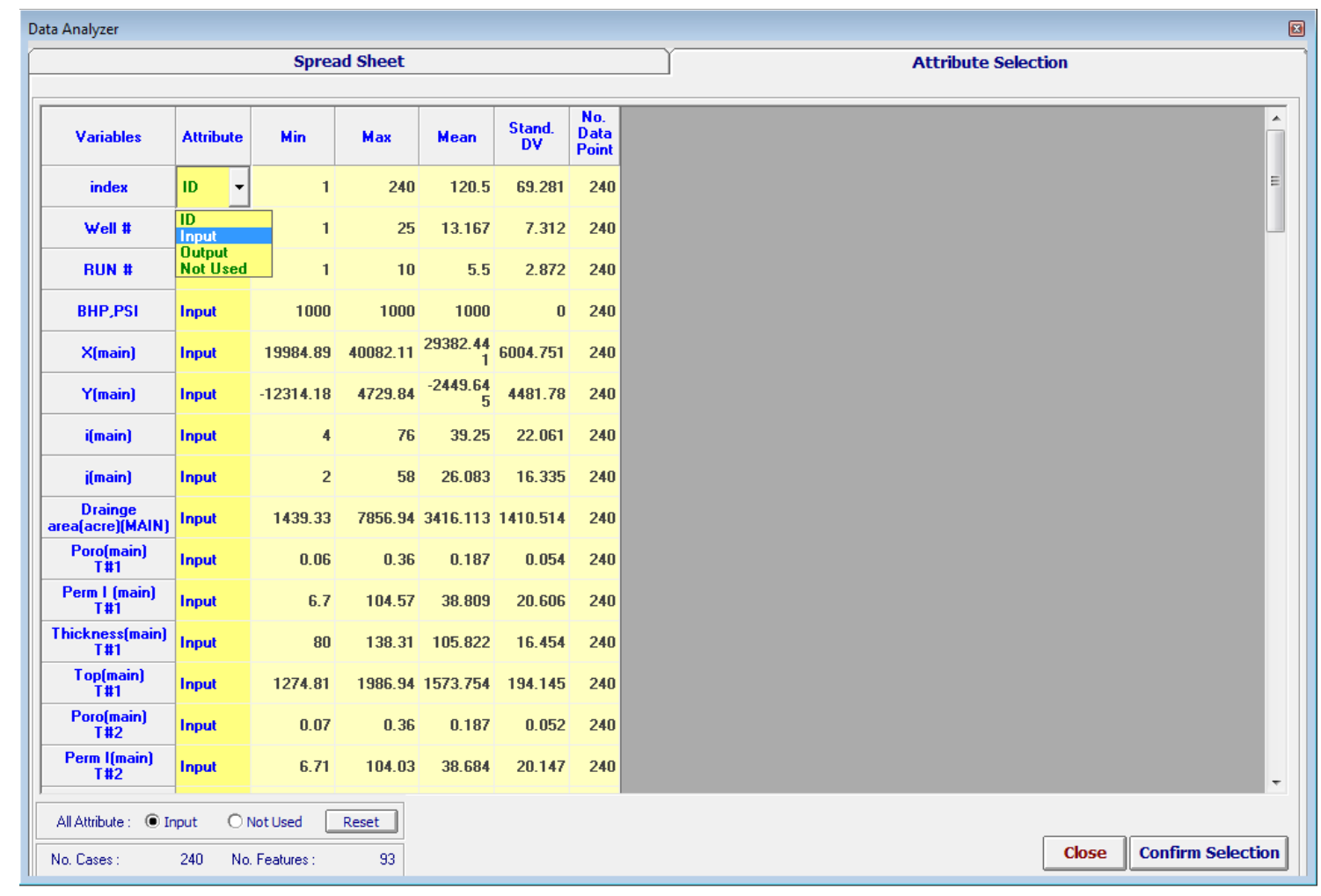

Figure 18- Attribute Selection for Imported Data Set 


\subsubsection{Key Performance Indicator}

It is a fact that all the imported parameters do not necessarily have equal impact on the output of the model. Therefore, screening the input data not only saves run time, but also provides a better understanding of the models' behavior. IDEA has some features to accomplish these kinds of analyses. In the "Data Mining” section of this software, you are able to perform some basic and advanced statistical analyses (Figure 19).

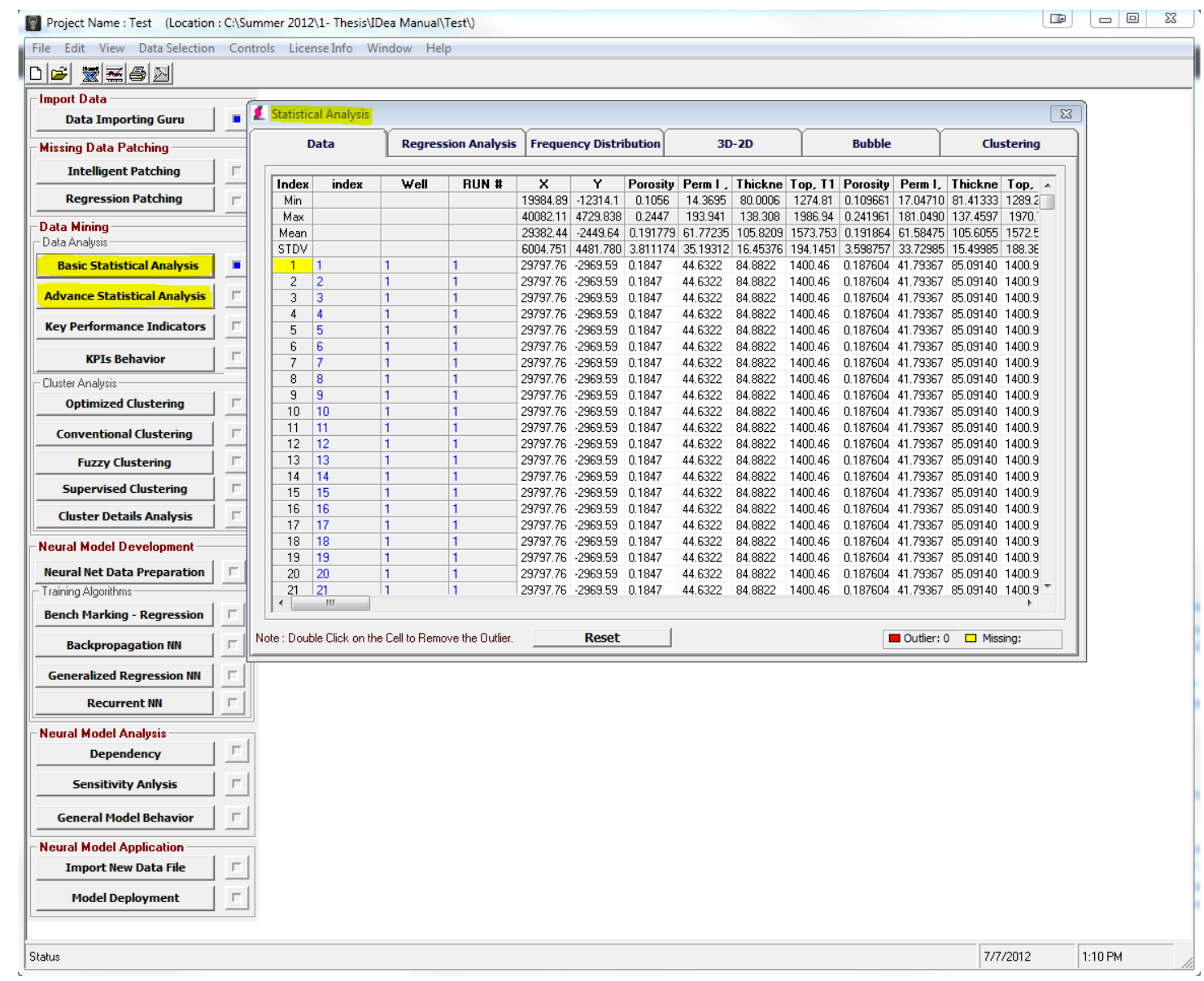

Figure 19- Statistical Analysis features of IDEA

One of the most important features of IDEA to rate the influence of parameters on the output is Key Performance Indicators (KPIs). This feature identifies the most influential parameters in any given process prior to modeling. The identification of KPIs makes the process of training, matching and validating SRM efficient. The result of this process is a tornado chart that indicates the percentage of degree of influence for each individual variable. Table 4 and Figure 20 summarize the results of KPI analysis for the first NN (Initialization model). As expected, the most high-ranking parameters for this model are permeability values for the target well and the offset wells. 
Table 4- KPI Analysis for the Initialization NN model

\begin{tabular}{|c|c|c|}
\hline Rank & Feature & $\%$ Degree of Influence \\
\hline 1 & Perm I, T1 & 100 \\
\hline 2 & Perm I, T2 & 98 \\
\hline 3 & Perm I, T3 & 82 \\
\hline 4 & Perm I, T4 & 57 \\
\hline 5 & Perm I(off1) T\#4 & 37 \\
\hline 6 & Perm I(off1) T\#3 & 33 \\
\hline 7 & $\mathbf{x}$ & 31 \\
\hline 8 & $x($ off1) & 31 \\
\hline 9 & Perm I(off3) T\#4 & 28 \\
\hline 10 & Perm I(off1) T\#2 & 25 \\
\hline 11 & Perm I(off3) T\#3 & 25 \\
\hline 12 & Top, T1 & 24 \\
\hline 13 & Top, t2 & 23 \\
\hline 14 & Perm I(off2) T\#3 & 21 \\
\hline 15 & Perm I(off1) T\#1 & 21 \\
\hline 16 & Top T3 & 21 \\
\hline 17 & Top(off1) T\#1 & 21 \\
\hline 18 & Top(off1) T\#2 & 20 \\
\hline 19 & Perm I(off2) T\#4 & 20 \\
\hline 20 & Other inputs & Less than $20 \%$ \\
\hline
\end{tabular}

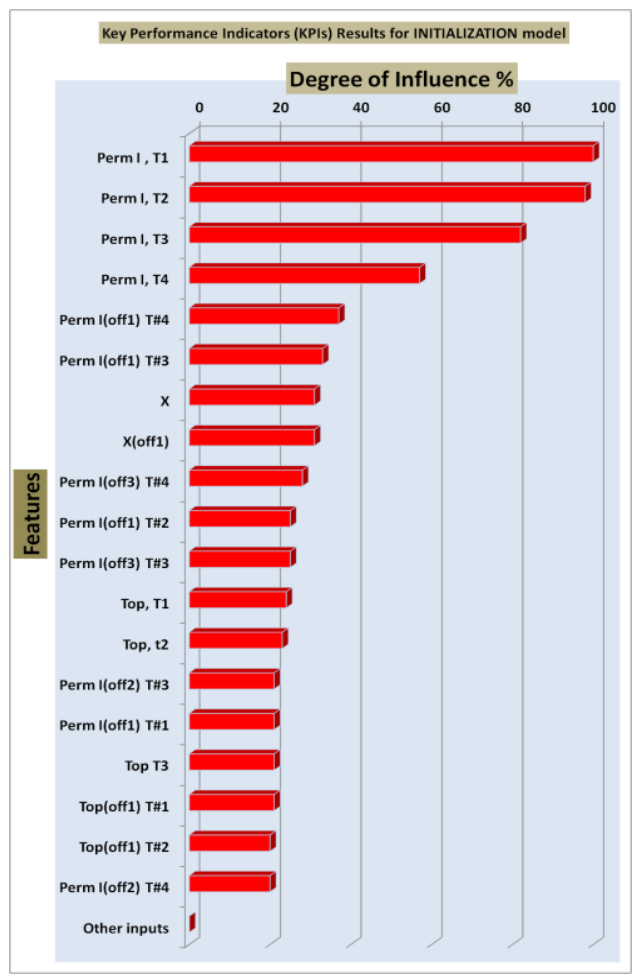

Figure 20- KPI Analysis, tornado plot for the Initialization model

Table 5 and Figure 21 show the results of KPI analysis for the Main NN model. In this model, dynamic parameters such as bottom-hole pressure, time and oil rate are the most important properties. 
Table 5- KPI Analysis for the Main NN model

\begin{tabular}{|c|c|c|}
\hline Rank & Feature & $\%$ Degree of Influence \\
\hline 1 & Oil Rate bbl/yr (t-1) & 100 \\
\hline 2 & BHP(psi) & 54 \\
\hline 3 & time & 52 \\
\hline 4 & Oil Rate bbl/yr Offset well \# 3, t-1 & 42 \\
\hline 5 & Oil Rate bbl/yr Offset well \# $1, \mathrm{t}-1$ & 41 \\
\hline 6 & Oil Rate bbl/yr Offset Well \# 2, t-1 & 35 \\
\hline 7 & Top, T\#1 & 13 \\
\hline 8 & Top, T\#2 & 12 \\
\hline 9 & Perm I, T\#1 & 12 \\
\hline 10 & Top T\#3 & 11 \\
\hline 11 & Perm I, T\#2 & 11 \\
\hline 12 & $X(o f f 1)$ & 11 \\
\hline 13 & Thickness, T\#2 & 11 \\
\hline 14 & Thickness, T1 & 10 \\
\hline 15 & Top(off1) T\#4 & 10 \\
\hline 16 & Top(off1) T\#1 & 10 \\
\hline 17 & Porosity, T\#4 & 10 \\
\hline 18 & Top(off1) T\#2 & 9 \\
\hline 19 & Thickness T\#4 & 9 \\
\hline 20 & Other Features & Less than $9 \%$ \\
\hline
\end{tabular}

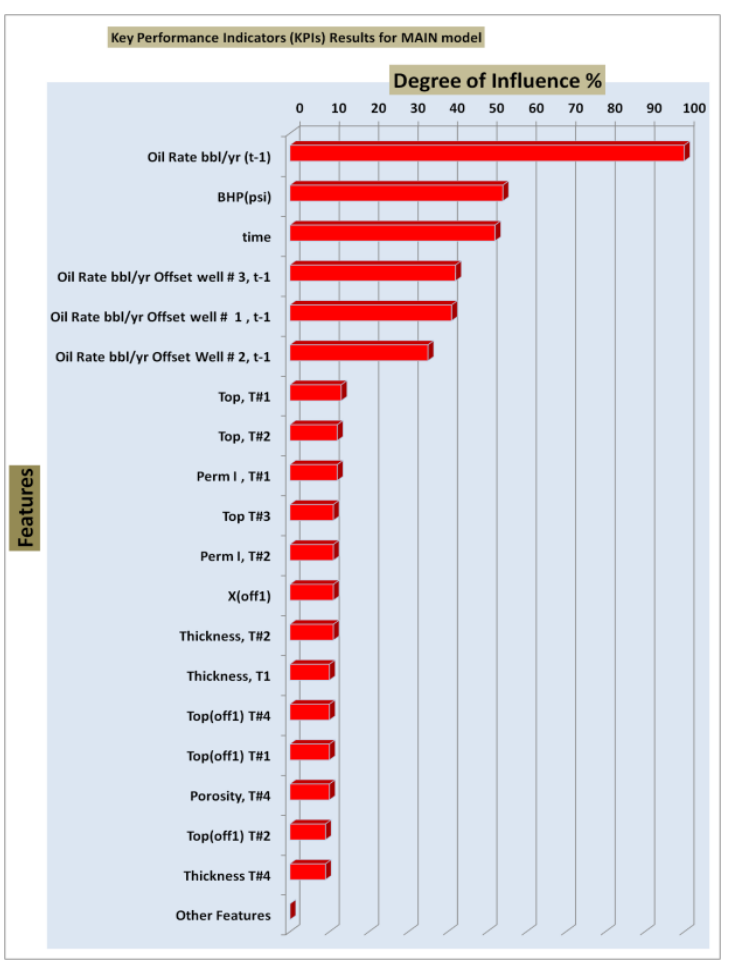

Figure 21- KPI Analysis, tornado plot for the Main NN model

After identifying the most important parameters for both models, the next step is to develop the NN models. The inputs of NN models are selected based on the provided information from the KPI analysis. Table 6 shows the selected inputs for the Initialization NN model, which 
only includes the static properties. The selected inputs (dynamic and static properties) are shown in tables 7 and 8.

Table 6- Selected inputs for the Initialization NN model

\begin{tabular}{|c|}
\hline Static Inputs \\
Main Well \\
\hline X \\
\hline Y \\
\hline Porosity, T1 \\
\hline Perm I, T1 \\
\hline Thickness, T1 \\
\hline Top,T1 \\
\hline Porosity, T2 \\
\hline Perm I, T2 \\
\hline Thickness, T2 \\
\hline Top,T2 \\
\hline Porosity, T3 \\
\hline Perm I, T3 \\
\hline Thickness, T3 \\
\hline Top,T3 \\
\hline Porosity, T4 \\
\hline Perm I, T4 \\
\hline Thickness, T4 \\
\hline Top,T4 \\
\hline Perm I, T4, Offset \#1 \\
\hline Perm I, T4, Offset \#2 \\
\hline Perm I, T4, Offset \#3 \\
\hline
\end{tabular}

Table 7- Dynamic inputs for the Main NN model

\begin{tabular}{|c|}
\hline Dynamic Inputs \\
\hline BHP(psi) \\
\hline time \\
\hline Oil Rate bbl/yr , (t-1) \\
\hline Oil Rate bbl/yr Offset well \# 1,(t-1) \\
\hline Oil Rate bbl/yr Offset Well \# 2,(t-1) \\
\hline Oil Rate bbl/yr Offset well \# 3,(t-1) \\
\hline
\end{tabular}


Table 8- Static inputs for the Main NN model

\begin{tabular}{|c|c|}
\hline \multicolumn{2}{|c|}{ Static Inputs } \\
\hline Main Well & Offset Wells \\
\hline X & X \\
\hline Y & Y \\
\hline Porosity, T1 & Perm I, T4 \\
\hline Perm I, T1 & Thickness, T4 \\
\hline Thickness, T1 & \\
\hline Top,T1 & \\
\hline Porosity, T2 & \\
\hline Perm I, T2 & \\
\hline Thickness, T2 & \\
\hline Top,T2 & \\
\hline Porosity, T3 & \\
\hline Perm I, T3 & \\
\hline Thickness, T3 & \\
\hline Top,T3 & \\
\hline Porosity, T4 & \\
\hline Perm I, T4 & \\
\hline Thickness, T4 & \\
\hline Top,T4 & \\
\hline
\end{tabular}

\subsubsection{Training, Calibration and Verification Sets of Data}

After passing the initial data analysis and selecting the proper inputs to build the SRM, the next step is to train the SRM and match its performance with the reservoir simulation model. At this step, SRM is trained to learn the reservoir simulation behavior and the fluid flow process in the reservoir. The main source of information for this process is the provided spatio-temporal database prepared in the previous steps.

The training process includes three different procedures: training (learning), calibration and validation. The database is then divided into three sets: training or learning set, calibration set and validation or verification set. The training set is the part of data shown to the NNs during the training process. The NNs are adapted to this set to match the provided outputs (reservoir simulation results). On the other hand, the calibration set is not used to adjust the outputs. This set is utilized to assure that any increase in accuracy over the training data set will lead to an increase in accuracy over a data set that has not been shown to the NNs before. This set of data 
is helpful to find out when the training should be stopped. If the error trend for the training data set has a decreasing trend, but the same error for the calibration set has a different trend, the NN is over-fitting and it is time to stop the training process. Finally, the verification set is the part of the database used to verify the predictability of the trained NN. This data set has not been used to train the NNs. It is worth mentioning that the elapsed time to perform the training process (learning, calibration and verification) is negligible compared to the reservoir simulation run-time.

The IDEA provides a data-partitioning algorithm, called Intelligent Data Partitioning, to set the training, calibration and verification shares of the database. Intelligent Data Partitioning presents a random picking out of the database. As mentioned, the spatio-temporal database was built based on the information from the ten simulation runs. The training, calibration and verification included $80 \%, 10 \%$ and $10 \%$ of the data in the database, respectively. It should be noted that the size of each partition, like many other things in the development of SRMs, is a function of the complexity of the problem at hand. There is no "one size fits all" but there are "rules of thumb" that can be followed. Besides the aforementioned verification part, another validation procedure was utilized in this study. The difference between these verification methods goes back to the type of data has been used to perform them. The details about this process, which is called "Blind Verification", come in the following sections in this chapter (Section 2.3.7).

\subsubsection{Training the SRM}

So far, two different NNs have been initiated to develop the SRM. The database was imported into the software, inputs were selected to train NNs and the database was divided into three parts. At this step, the SRM was ready to begin the training procedure. The training algorithm used in this study is back propagation method. Back propagation networks always consist of at least one hidden layer. Krose and Smagt (1996) mentioned in their book that only one hidden layer is enough to approximate any function with finitely many discontinuities to arbitrary precision. Therefore, one hidden layer was used in this study.

The number of neurons in the hidden layer is completely our choice and there is no rule to determine the best possible number. If the number is too large, it will encourage the network to memorize the input patterns instead of learning the prototype of reservoir simulation. On the other hand, a hidden layer with a too small number of neurons will drastically increase the number of iterations needed to train the model. The IDEA always has a suggested number of 
neurons, which depends on the number of inputs. In this study, the recommended numbers of IDEA were used, which are 20 and 50 for the Initialization and Main models, respectively.

Figures 22 and 23 show the NN structures for the Initialization and Main models. These graphs portray three different layers of NNs. The input layer includes the parameters chosen in the input selection part. The hidden neurons were suggested by IDEA and the output of the models is oil rate. Different layers were connected by weight vectors, and these weights changed constantly during the training to reach the desired output (reservoir simulation results).

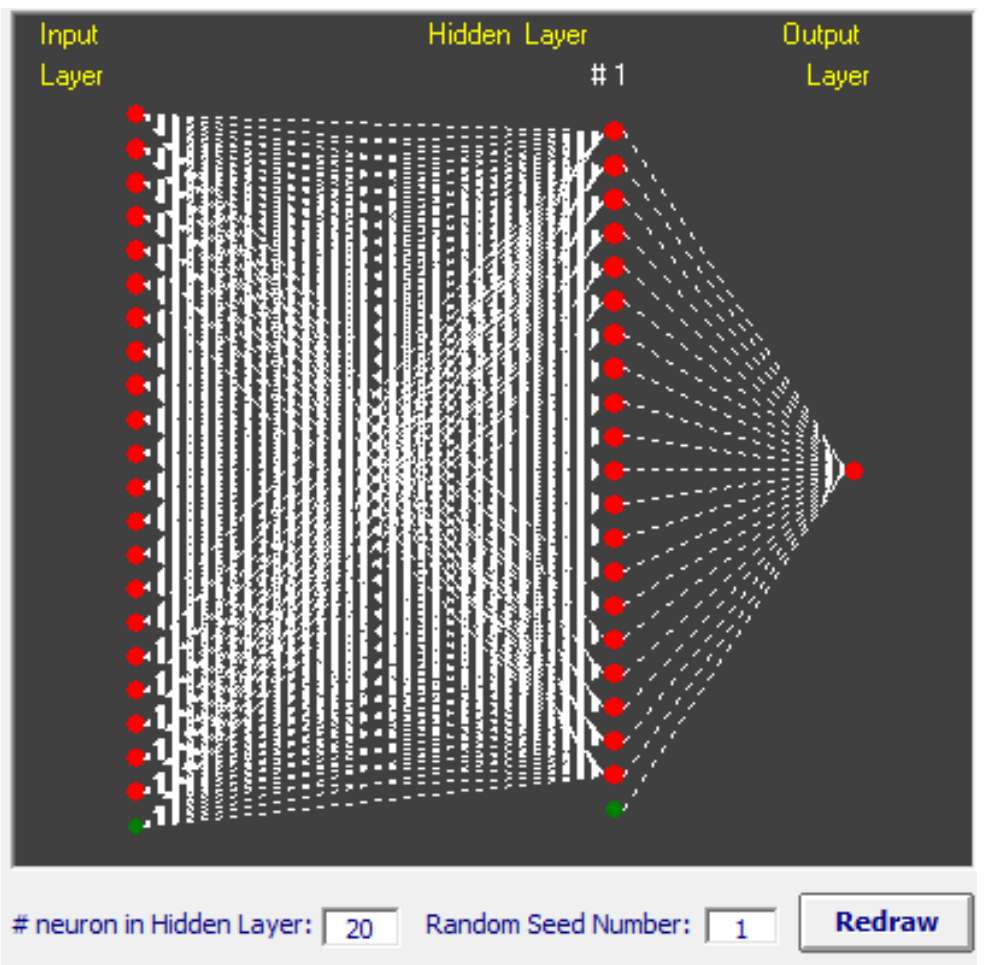

Figure 22- The Initialization NN model structure with 20 neurons in the hidden layer 


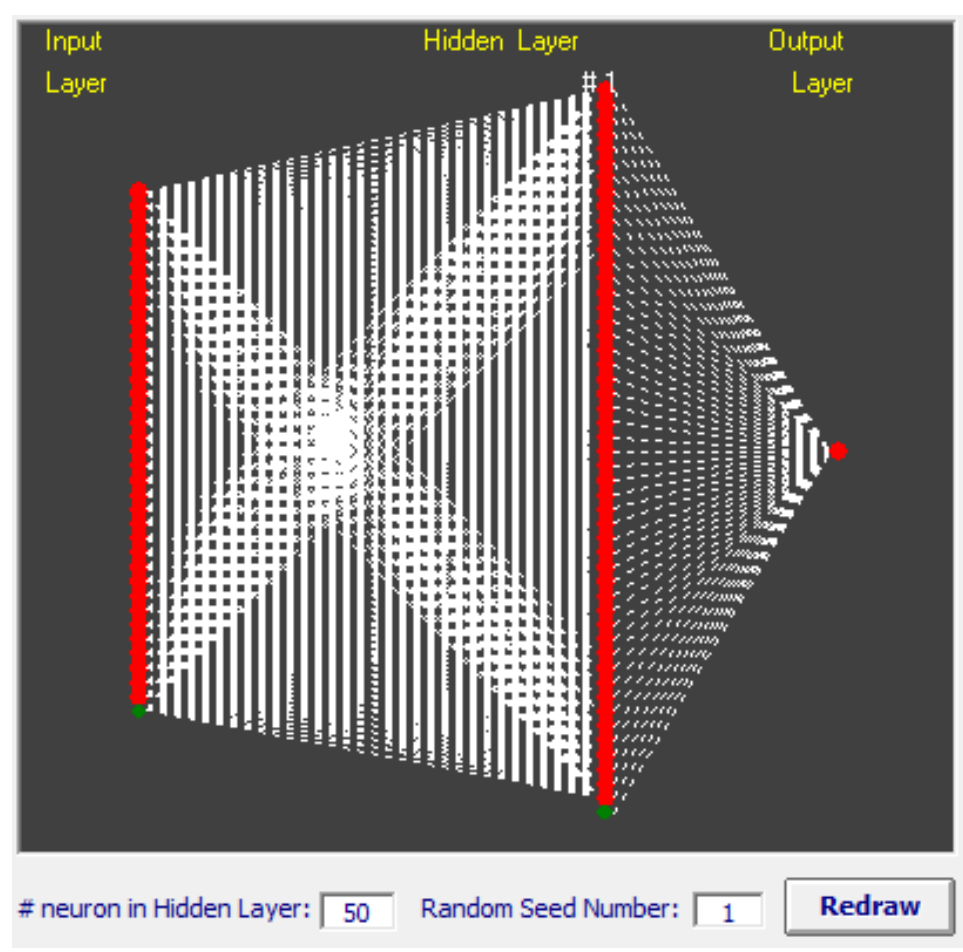

Figure 23- The Main NN model structure with $\mathbf{5 0}$ neurons in the hidden layer

Figure 24 is a snapshot of the training procedure. The graph shows the changes in the errors of the training and calibration data sets. The error is calculated by subtracting the SRM predicted oil production from the measured oil production of the reservoir simulator.

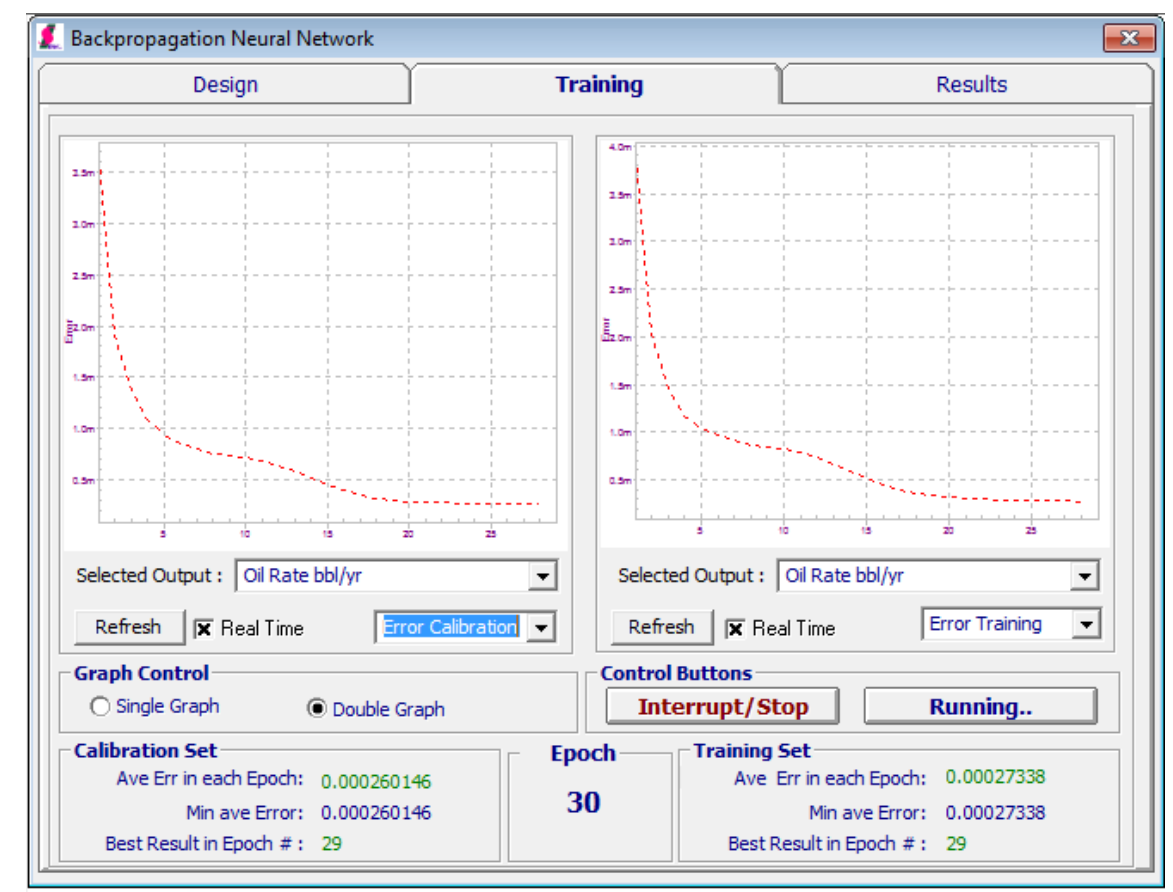

Figure 24- A snapshot of the training procedure 
Figures 25 to 30 show the results of the training process for the learning, calibration and verification sets in the Initialization and the Main NN models. The graphs are scatter plots comparing simulator results with the SRM predictions.

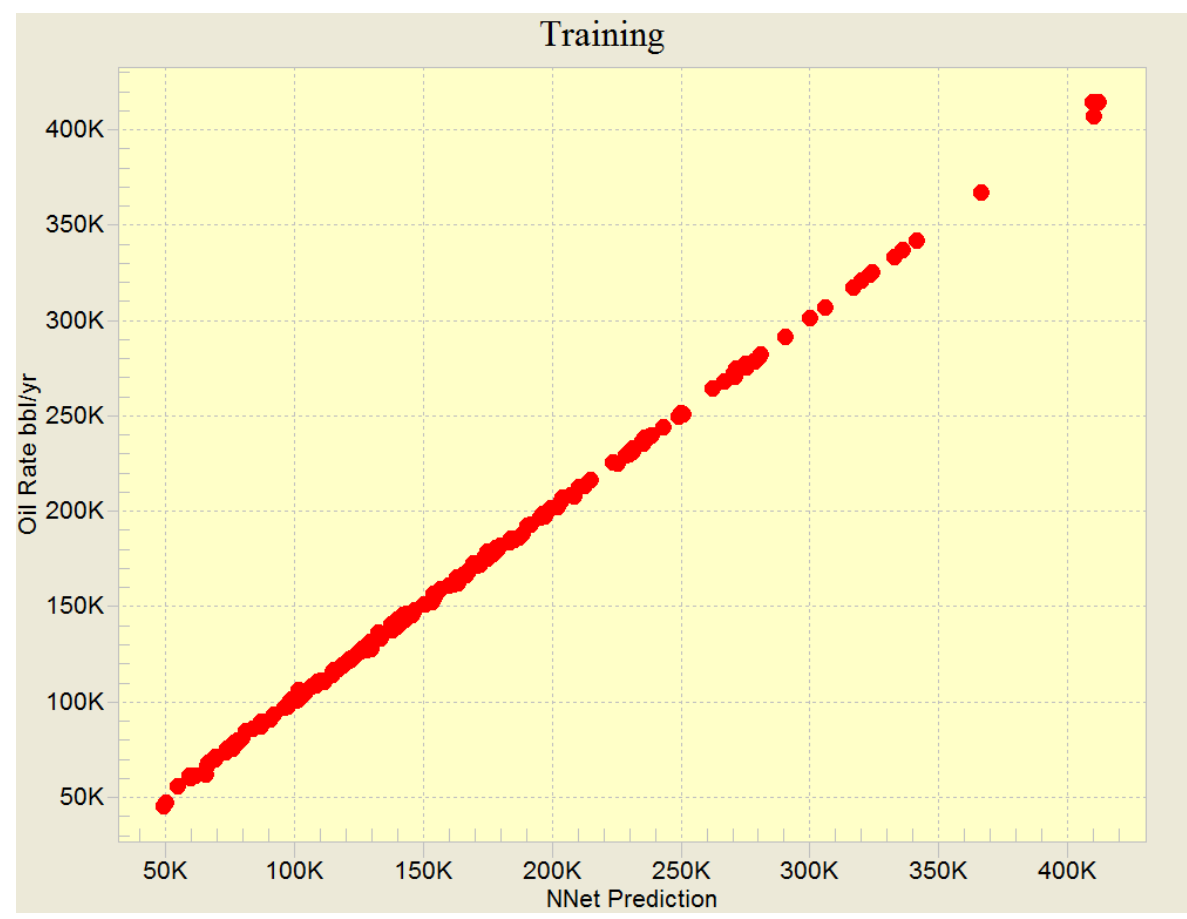

Figure 25-Scatter plot of Training set results in the Initialization Model

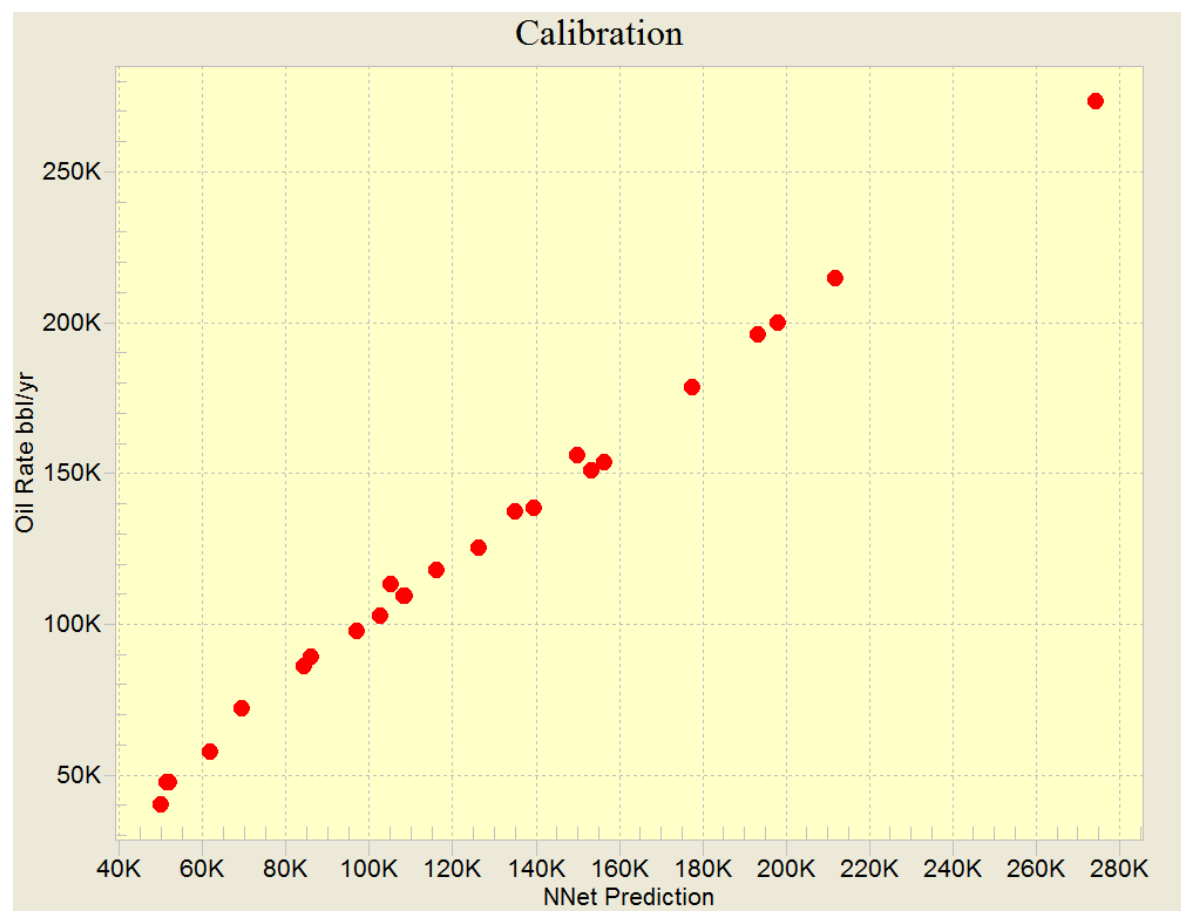


Figure 26- Scatter plot of Calibration set results in the Initialization Model

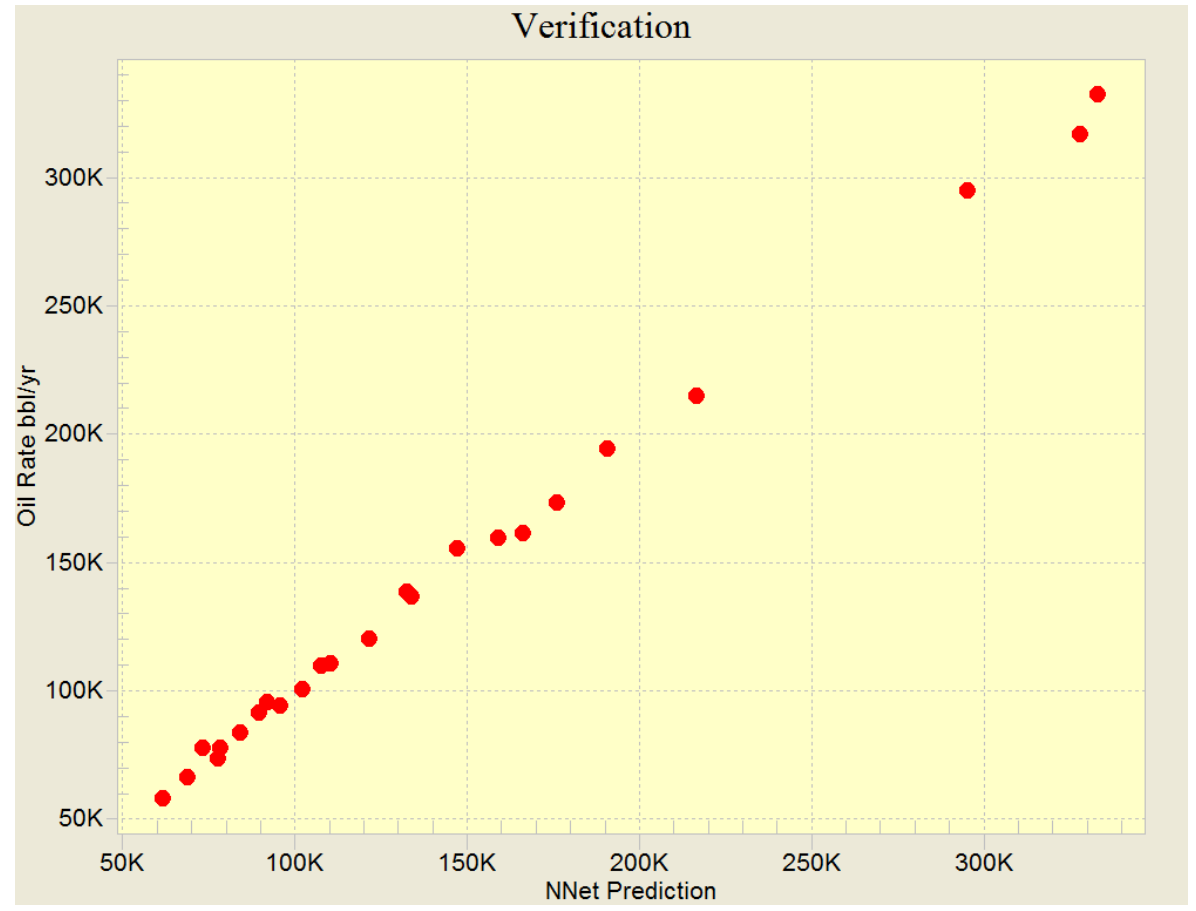

Figure 27- Scatter plot of Verification set results in the Initialization Model

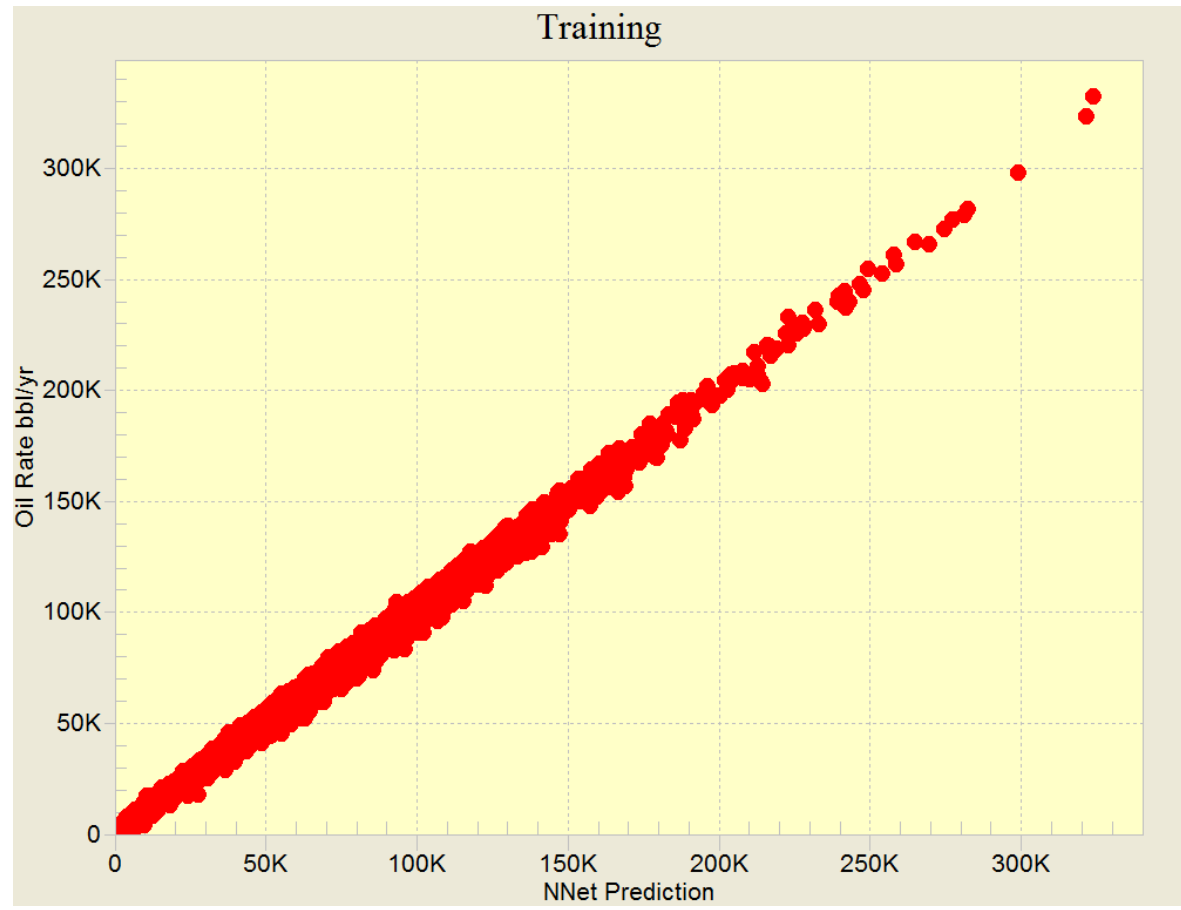

Figure 28- Scatter plot of Training set results in the Main Model 


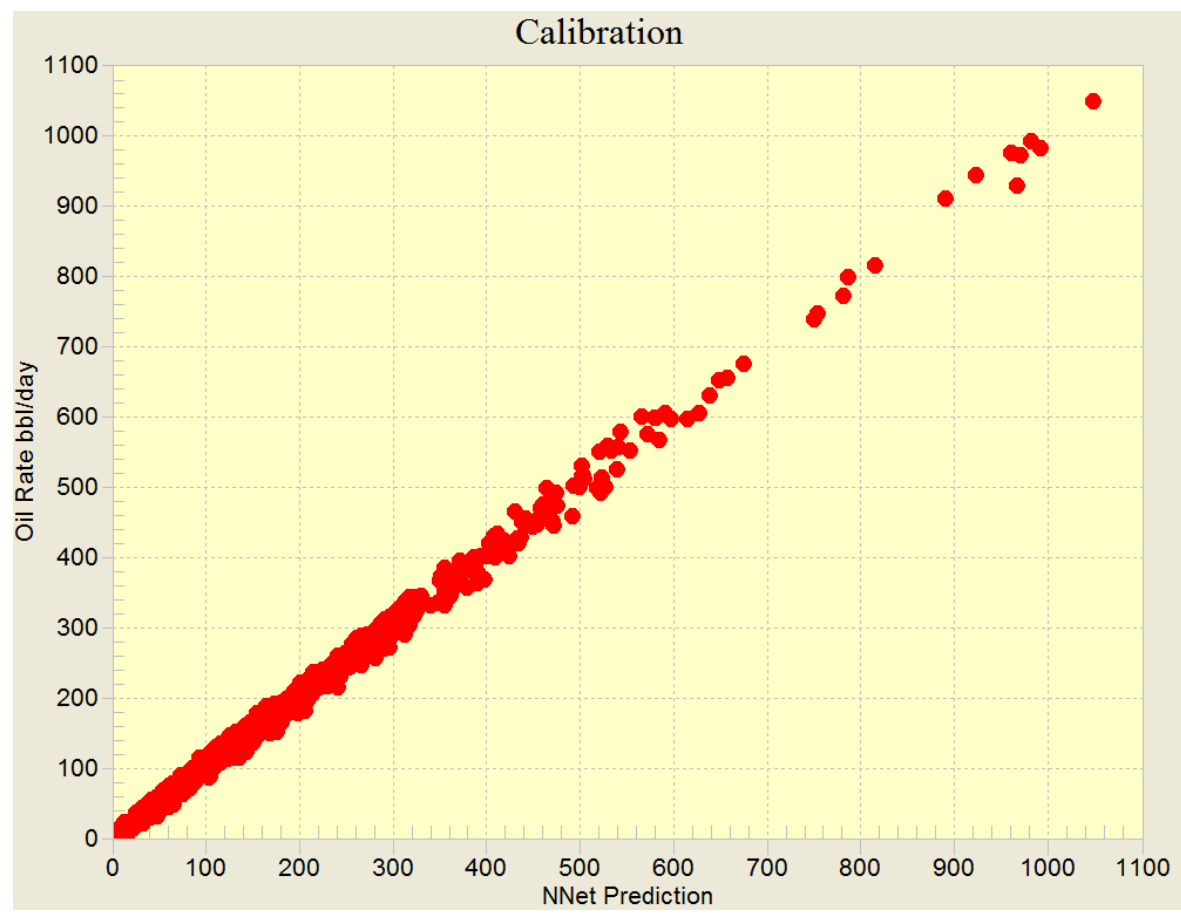

Figure 29-Scatter plot of Calibration set results in the Main Model

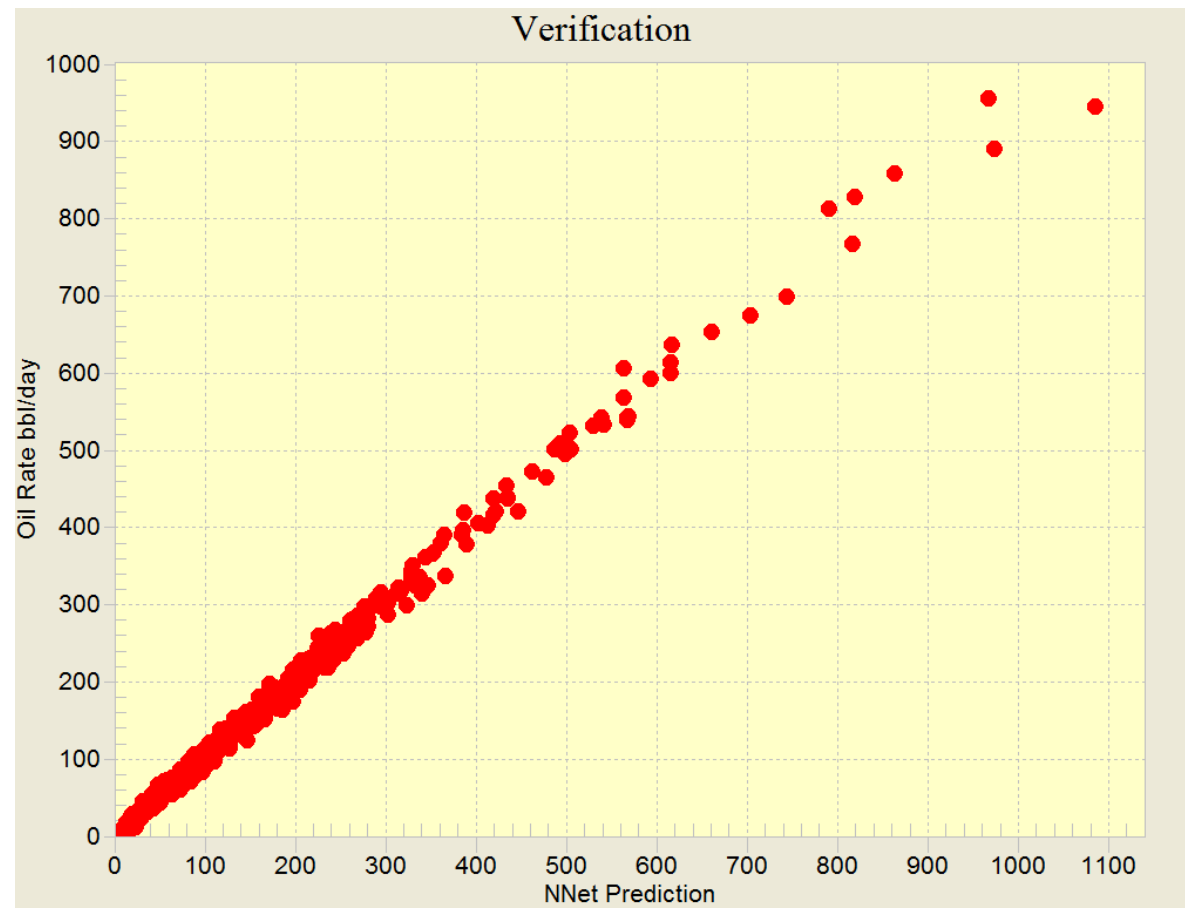

Figure 30- Scatter plot of Verification set results in the Main Model 


\subsubsection{Validation the SRM by a Blind Realization}

The trained SRM was validated against a complete blind realization of the reservoir. Therefore, the $11^{\text {th }}$ simulation run was made where the permeability realization was completely different from the ten runs that were used for training the SRM. This blind testing set is a complete realization of the reservoir, while the verification set used in the training process was a randomly selected portion (10\%) of ten realizations. The same process of data extraction was applied. It is also worth mentioning that this blind realization should honor the range of permeability, which was used to train the SRM. Finally, the trained SRM was applied to predict the oil rate from the blind realization inputs. Figure 31 and Figure 32 depict the permeability distribution for the blind realization. The results of the blind verification run compared to the reservoir simulator values are in Chapter 3.

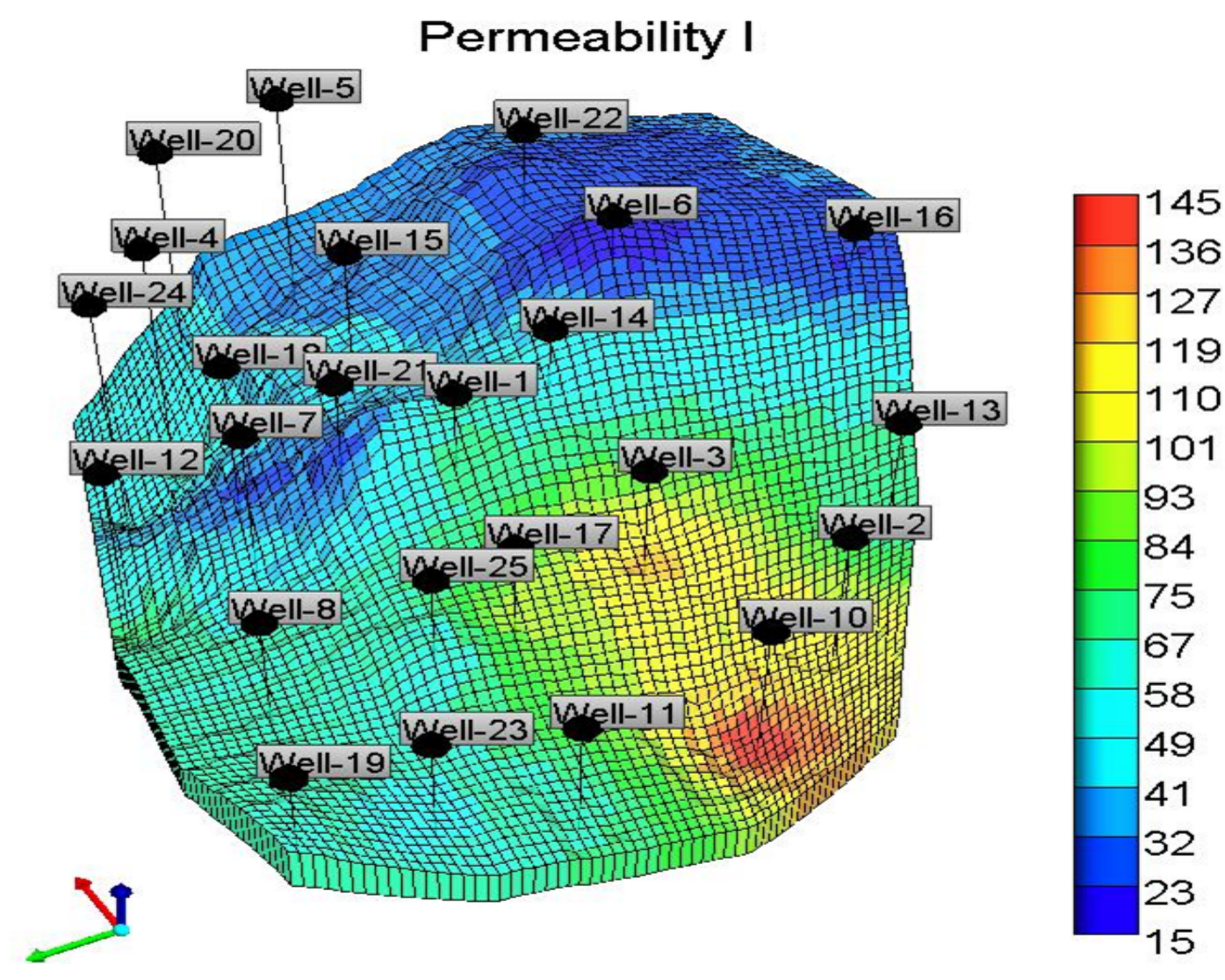

Figure 31- 3D view of permeability map for the blind verification realization 


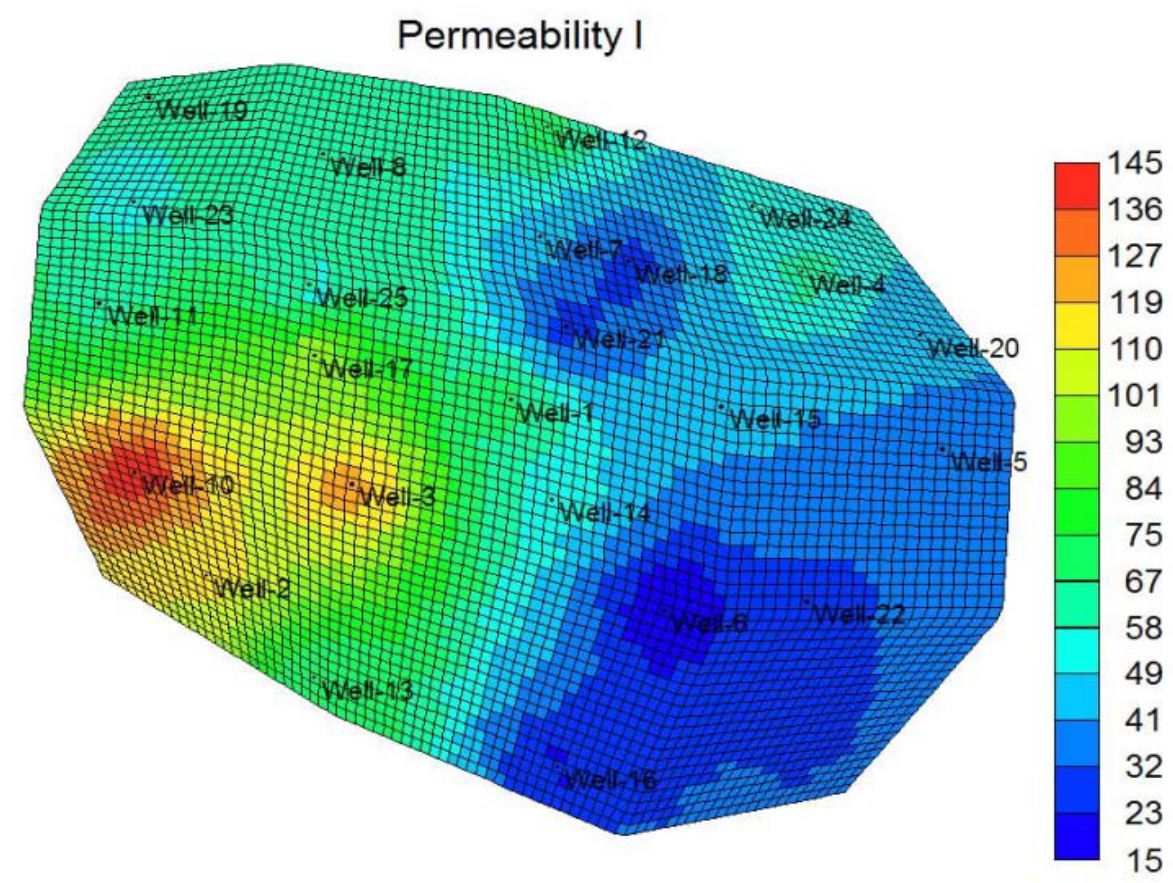

Figure 32- 2D view of permeability map for the blind verification realization

\subsection{History Matching}

At this step, the SRM is trained and is ready to replicate the reservoir simulator performance. The history match process could be described as a procedure where we try to find a replica for the reservoir based on real production history. By comparing the results from the SRM and the real data, if the production rate that comes from SRM does not match the values from the field, we conclude that the reservoir characteristics may not be correctly calculated and they must be modified in order to get the match.

In order to accomplish the history match, permeability values at each defined tier were adjusted. The oil rates predicted by the SRM were compared against the real production rates. This procedure was repeated until an acceptable match in each well was obtained.

At the first step of the history match process, objective functions (OF) were defined. The following equations are of the most common objective functions used for the history match process (Computer Modelling Group Ltd., 2011). This objective function measures the relative difference between the SRM results and measured production data. Equation 1 calculates the differences for a single well. The subscripts $i$ and $t$ represent well and time respectively. $N t(i)$ is the total number of measured data points (in this study, 30 annual oil rates for each 
well). $Y_{i, t}^{S}$ are the predicted production by SRM and $Y_{i, t}^{m}$ are measured production data. $\Delta Y_{i}^{m}$ is the scale calculated by subtracting the maximum and minimum of measured production data for well $i$. In a real case, there is measurement error and it should be considered in the calculation, but we assumed there was not this kind of error in this study.

Equation 1-Individual Well Objective Function

$$
O F_{i}=\frac{\sqrt{\frac{\sum_{t=1}^{N t(i)}\left(Y_{i, t}^{s}-Y_{i, t}^{m}\right)^{2}}{N t(i)}}}{\Delta Y_{i}^{m}} \cdot 100 \%
$$

It is common to define a global objective function in order to have calculations in the field level as well. Equation 2 describes the global objective function using the well level objective function, which was defined in Equation 1 . Here $O F_{\text {global }}$ is the global objective function, $Q_{i}$ is the objective function for well $i$, and $N w$ is the total number of wells (24 wells in this study). In practice, it is also common to consider that the quality and importance of measured data may be different for some specific wells. $w_{i}$ is the defined weight for well $i$. In this study, all the wells were considered equally important and the weight coefficient is one for all of them.

Equation 2-Global (Field) Objective Function

$$
O F_{\text {global }}=\frac{1}{\sum_{i=1}^{N_{w}} w_{i}} \sum_{i=1}^{N w} w_{i} O F_{i}
$$

Our manual history match process has two steps, which helps to save a lot of time. In the first step, we try to get a match just for the first year of the production by using the first NN model (Initialization). It starts with the initial guess for permeability at each tier for all wells. The initial guess can be any value within a reasonable range (for instance, the base case permeability values are a good start).

Starting with the well $i$ ( $i$ can be any of the wells) SRM calculates the first year of the production. If the results are not desired it is easy and fast to tune the permeability values at the different tiers to acquire the reasonable match. The matching process for the first year of production is performed for all the wells. It should be noted that the permeability value at the 
first tier (well block) is the most influential parameter to obtain a match. In addition, the permeability value at the fourth tier of the offset wells is an input for the SRM. That means after achieving a match for well $i$, the adjusted value of permeability should be used to predict the output of the wells that have well $i$ as their offset well. Therefore, after adjusting each permeability value, the adjusted value should be updated in the database. Figure 33 explains the approach used in this study to accomplish the history match process using the SRM. The left side of this flowchart explains the first year of production match.

The first year of the production is matched for all the wells. Consequently, there is a set of permeability values at this step. This set of permeability values feeds the next step of history match, which obtains a match over the whole range of the production including the first year. The second step has two main differences compared with the first step. In this step, instead of a random initial guess we are using the set of permeability values coming from the previous step. Based on the experiences in this study, the final matched values of permeability are close to the values of adjusted permeability coming from the matching process for the first year of production. Therefore, in the second phase of the matching process the final answer is strictly close to the used initial guesses. In addition, this step covers the entire range of production, which is 30 years. After each calculation, the objective functions (well and global) are measured.

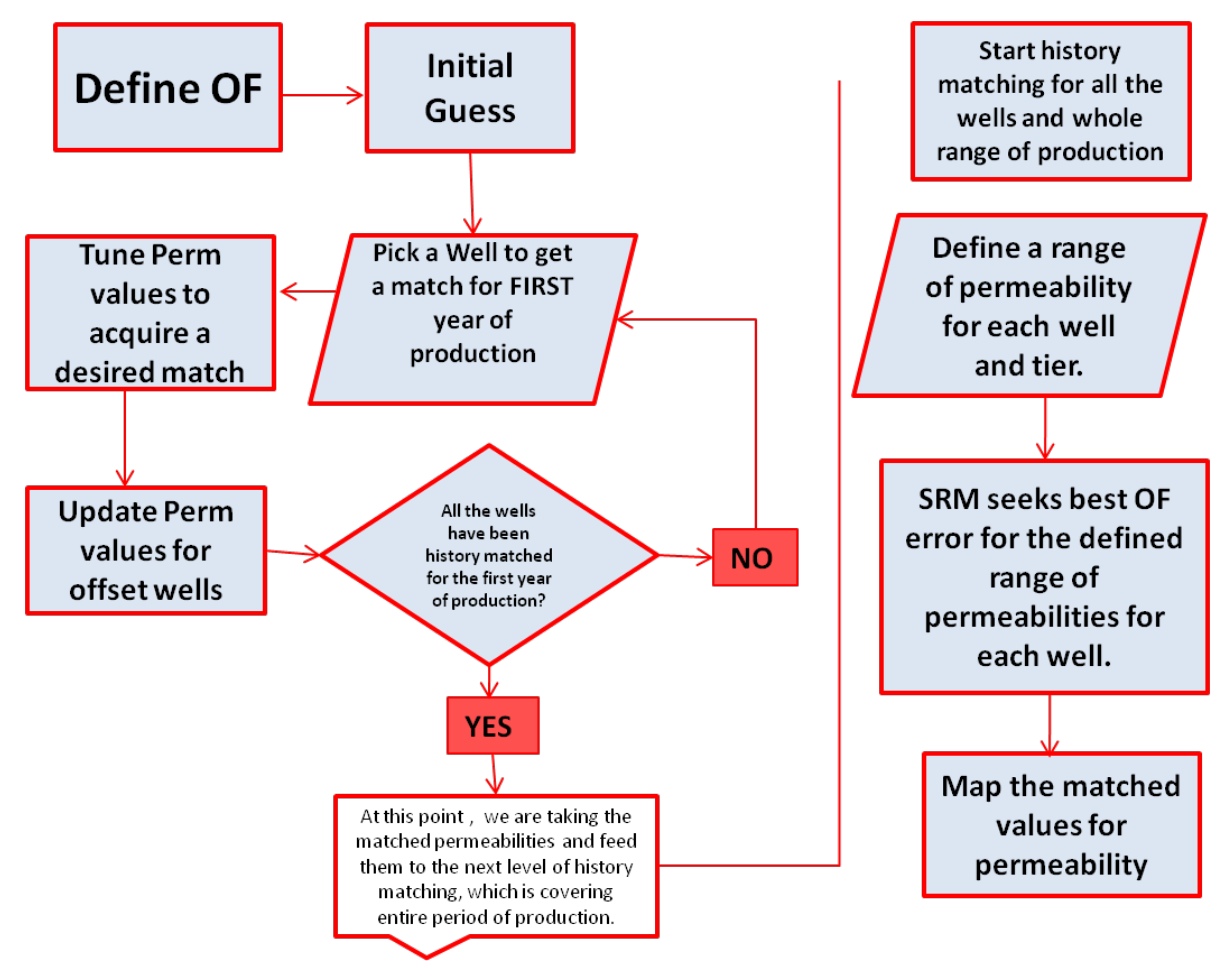

Figure 33- A flowchart explaining the manual history match process in this study 
By having a final set of permeability values, a permeability distribution map can be made. The matched and actual permeability maps are given in Chapter 3.

Each run of the developed SRM took less than one second to perform in a personal computer with $2.83 \mathrm{GHz}$ processor speed. On the other hand, the elapsed time to run the simulation model using the same computer was about one minute. To perform the history match without using SRM, over 900 simulation runs would be necessary. Although the number of simulation runs in a manual history match is not the optimum number, the optimization methods can be utilized to reduce the number of simulation runs. However, to train and validate the SRM only 11 simulation runs were used. The only time consuming part of SRM development was the preparation of spatio-temporal database. In addition, the time spent to train the NN models with the aforementioned personal computer was less than two hours. In the following chapter, by presenting the results of SRM, the accuracy of SRM will be discussed. 


\section{Chapter 3}

\section{Results and Discussion}

\subsection{Results}

This chapter intends to present the results for one of the wells (well \# 20) for different steps of developing and applying the SRM. The results for the rest of the wells are available in Appendix C. Figure 34 shows the results after the training process; the chart portrays the oil rate profile for 30 years of the production comparing SRM results with the simulator outputs. In this report, the blue squares represent the SRM and the red line with stars shows the simulator results. It can be seen that SRM can reproduce the simulator results with high accuracy.

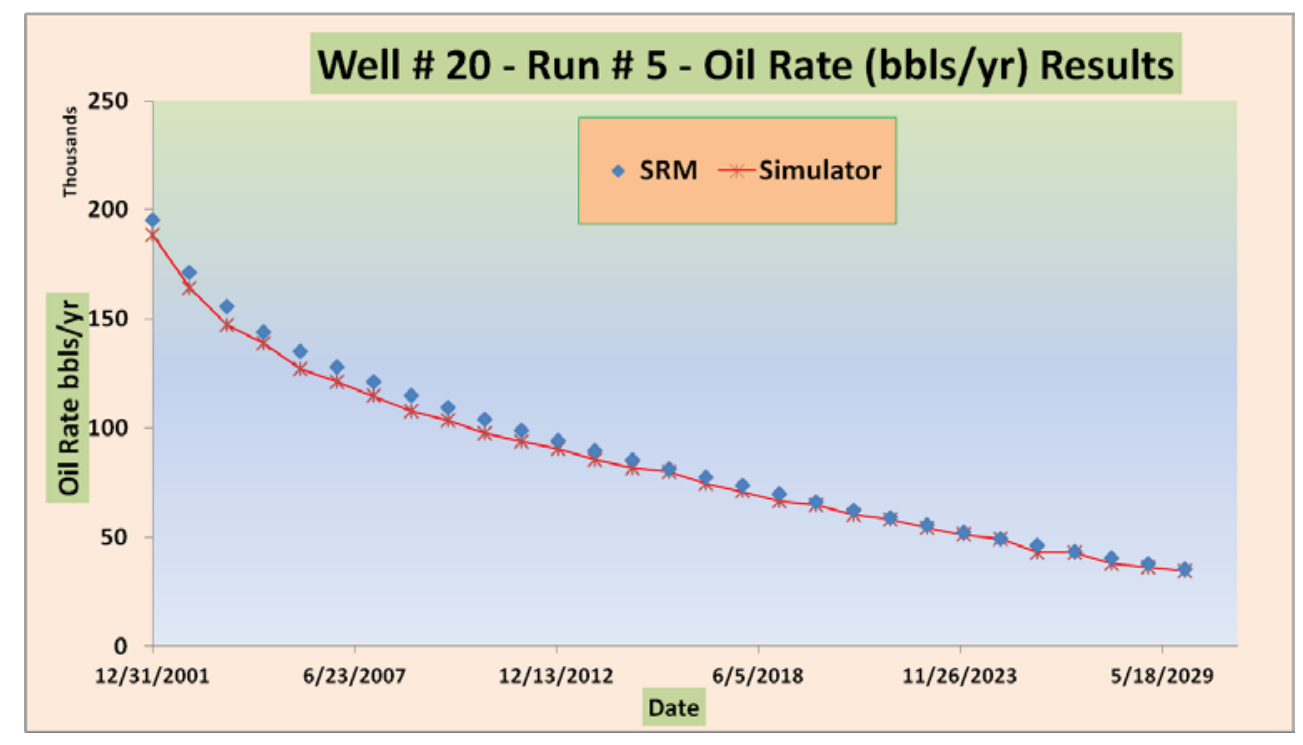

Figure 34- Training results well \# 20

Figure 35 shows the results of the blind verification realization. As was mentioned before, a blind realization was used for testing the SRM with a realization set which has not been seen by the SRM. Therefore, this graph shows the potential of SRM to predict a realization performance out of the training dataset. 


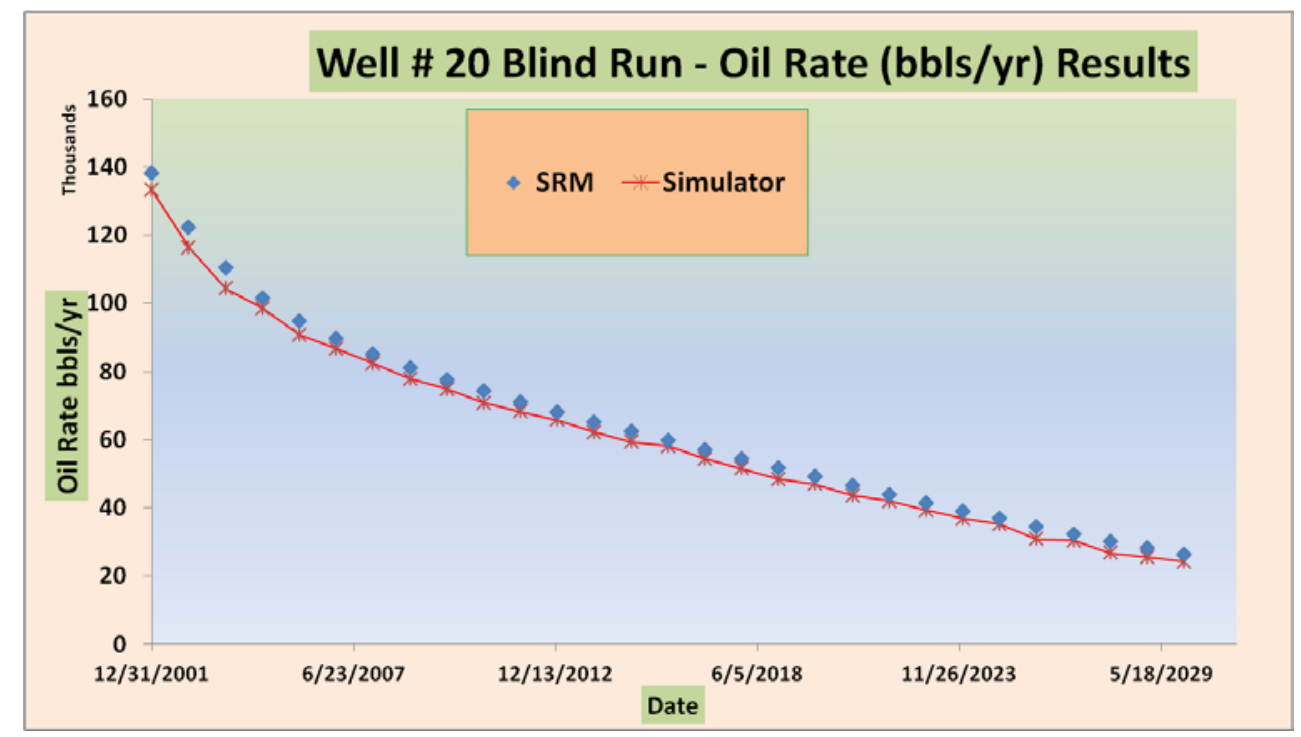

Figure 35- Blind run results for well \# 20

Finally, Figure 36 shows a snapshot of the history match results for this well. This graph is the comparison of the SRM with the measured production data.

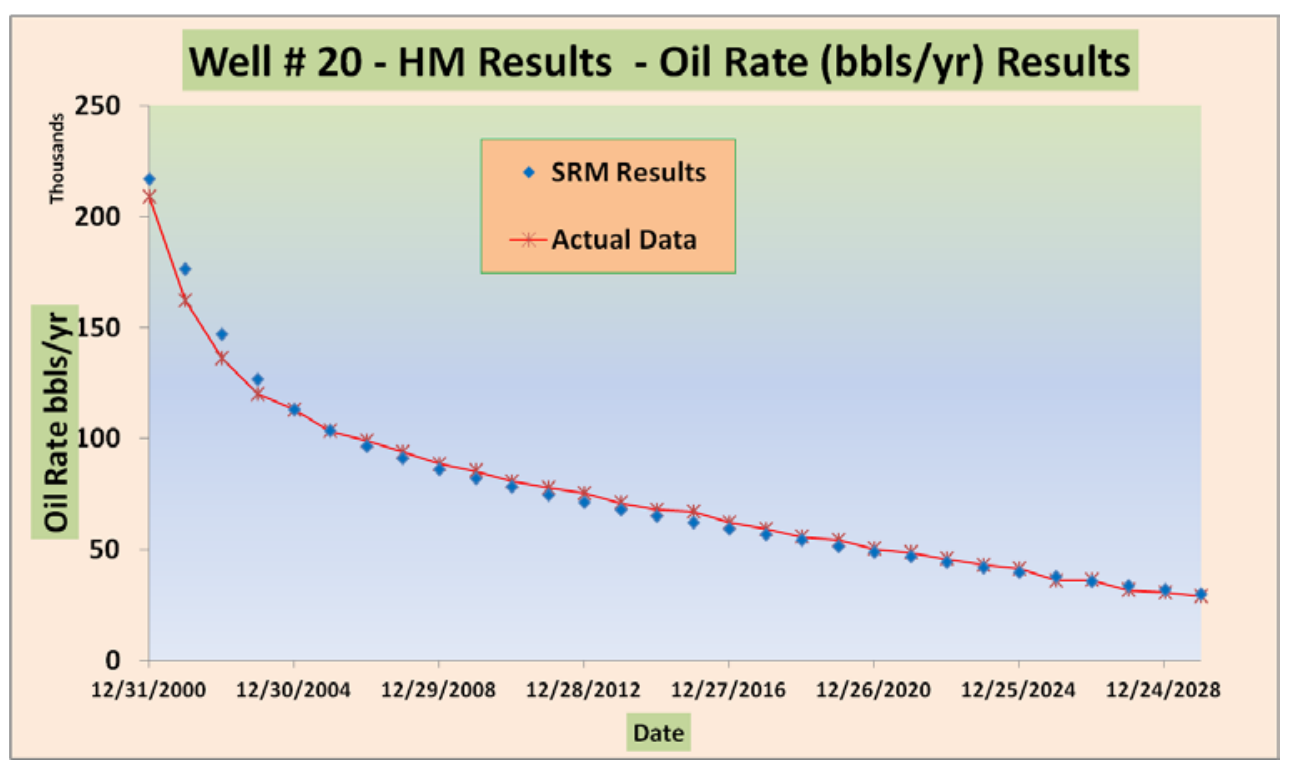

Figure 36- SRM-Based History Matching Results for well\# 20

The matched permeability values are shown as a permeability distribution map in Figure 37. The right side of this figure pictures some shots of the matched permeability map, while the left side shots are the actual permeability distribution. In addition, Figure 38 shows the error between the actual permeability and the matched one. 


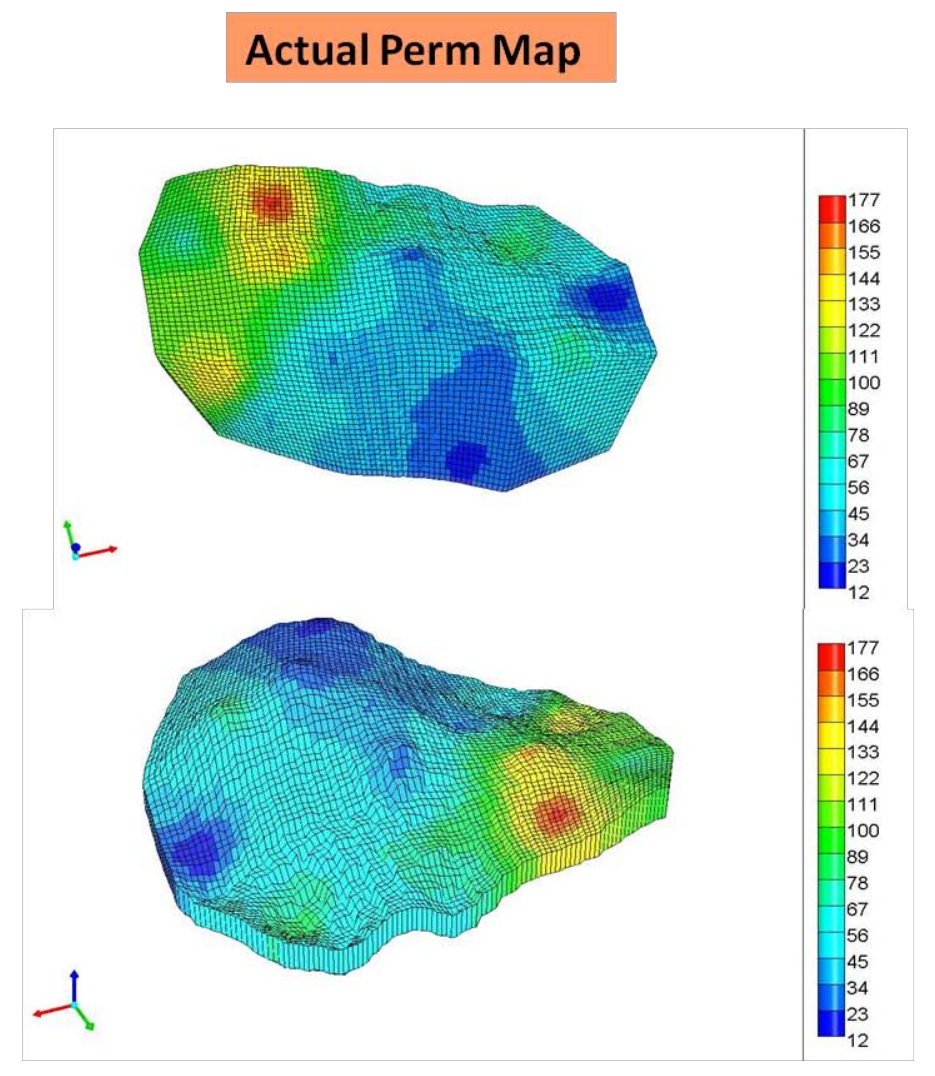

\section{Matched Perm Map}

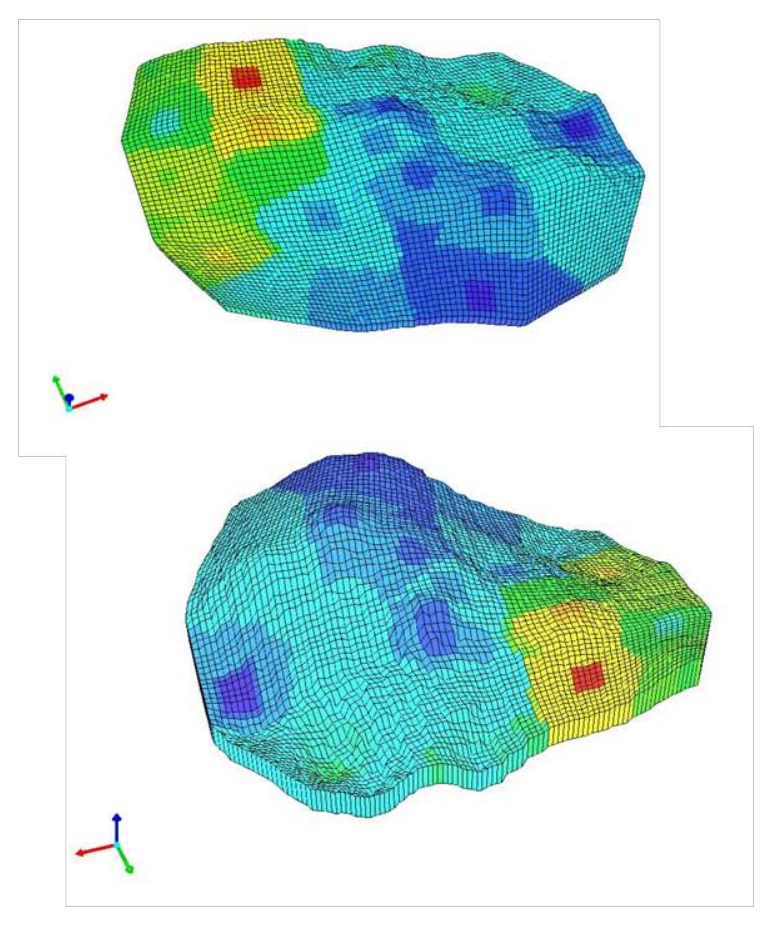

Figure 37- comparison of matched and actual Permeability distributions

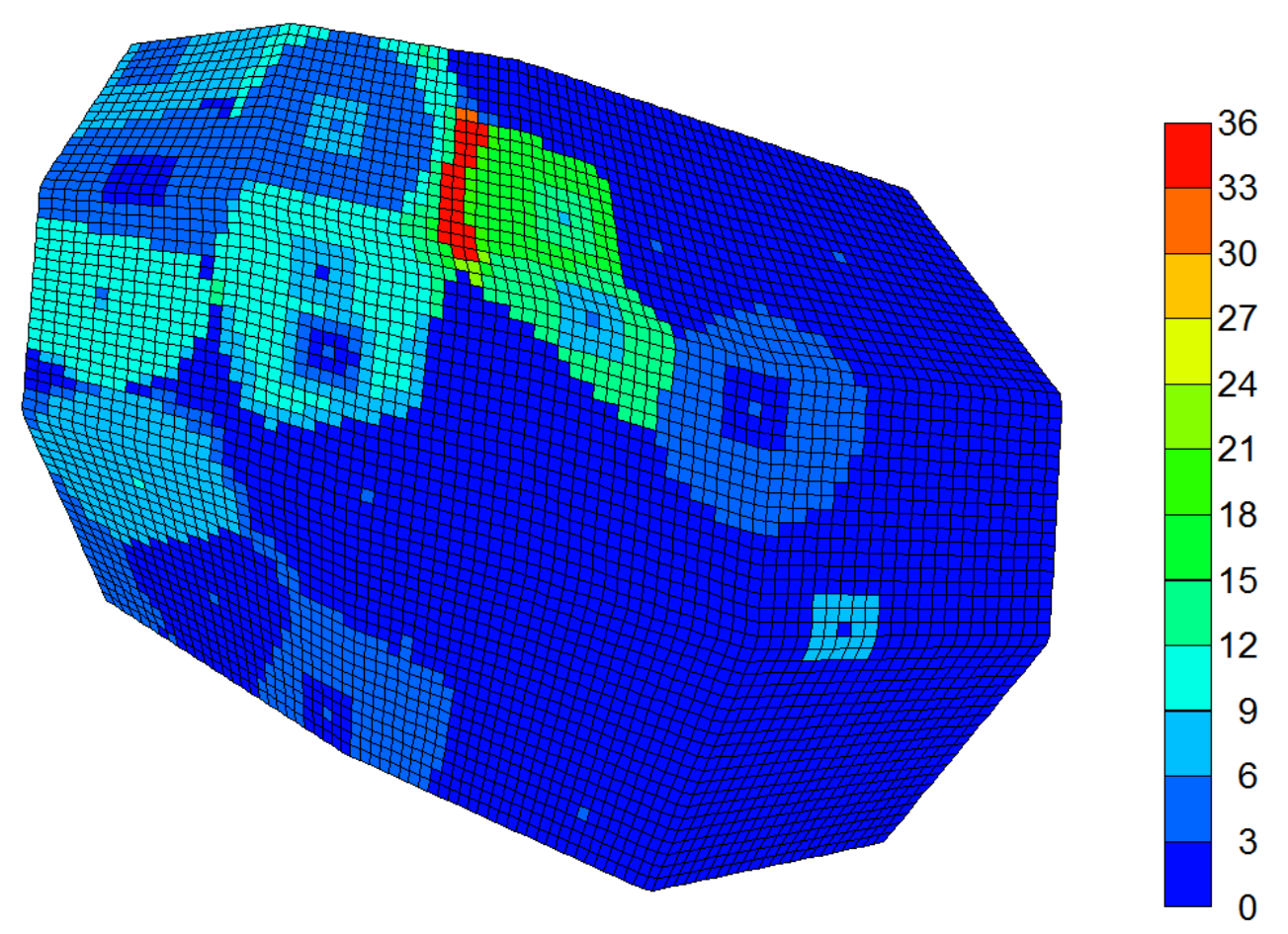

Figure 38- A snapshot of error distribution of permeability 


\subsubsection{Importing the matched map into the simulator}

In this study, an SRM was developed to perform the simulator's (CMG-IMEX) role. We were curious to see the results of the simulator after using the matched permeability map coming from the developed SRM. Figure 39 shows the field cumulative production results of the simulator after importing the matched permeability map from the SRM into the simulator. This graph compares the simulator results with the actual field cumulative production. The blue dashed curve represents the simulator results after using the matched permeability map, and the red line shows the actual performance. It is clear from the graph that there is a good match between the simulator and field results (the curves are overlapped).

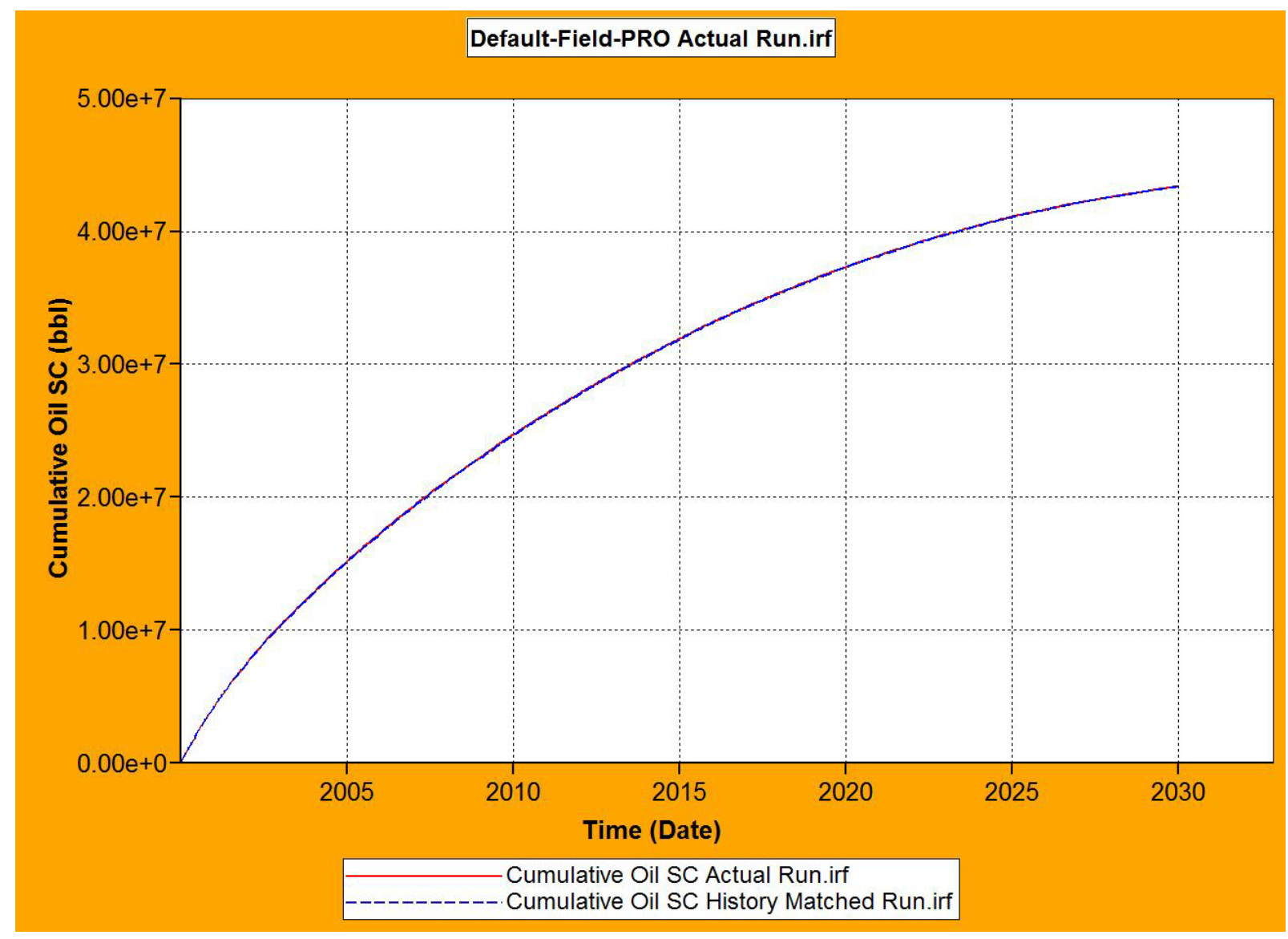

Figure 39 - Comparison of field cumulative production between actual data and the simulator performance. The permeability map is the matched permeability map coming from the SRM.

Figure 40 and Figure 41 depict the oil rate profile and cumulative oil production for four different wells. These figures compare the actual field performance to the simulator results that used the matched permeability map. Similarly, the blue-dashed curve and red curve represent the simulator and actual values, respectively. 


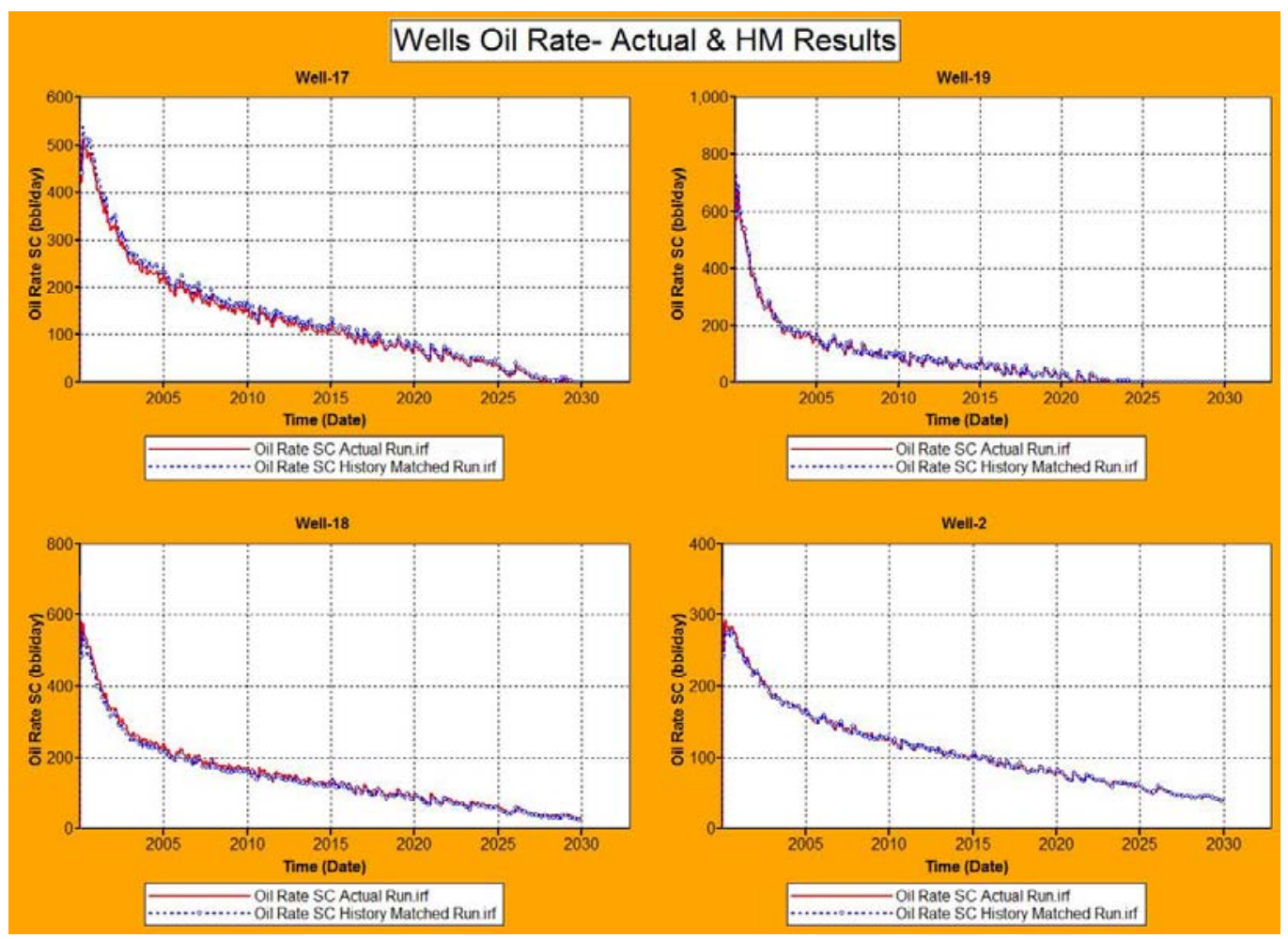

Figure 40- Comparison of oil rate production between actual data and the results coming from simulator after applying the matched permeability map from SRM

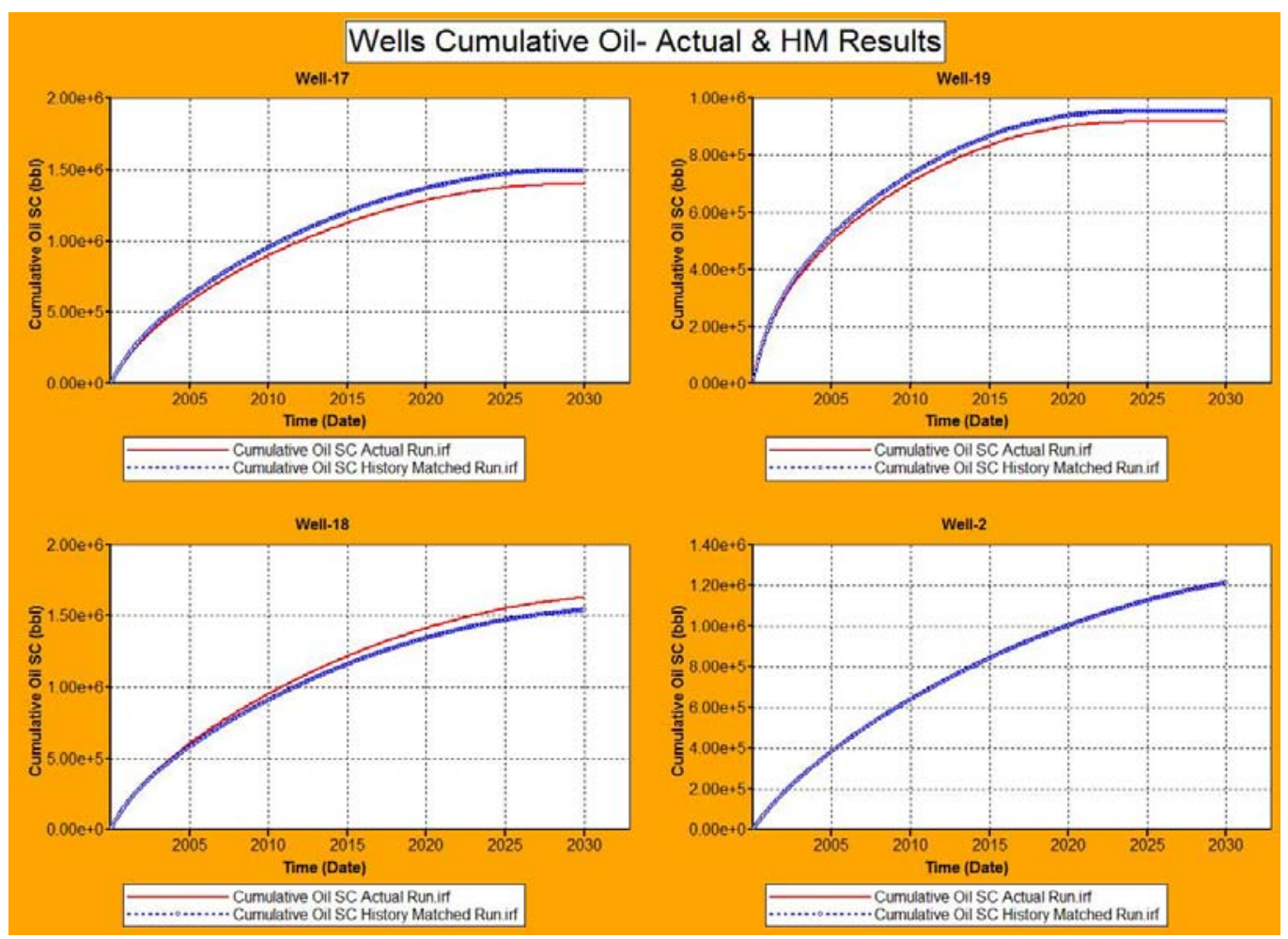

Figure 41-Comparison of cumulative production between actual data and the results coming from simulator after applying the matched permeability map from SRM 
Figure 42 shows the error distribution of the results after the history match process. Figure 43 also shows the error frequency distribution of these results.

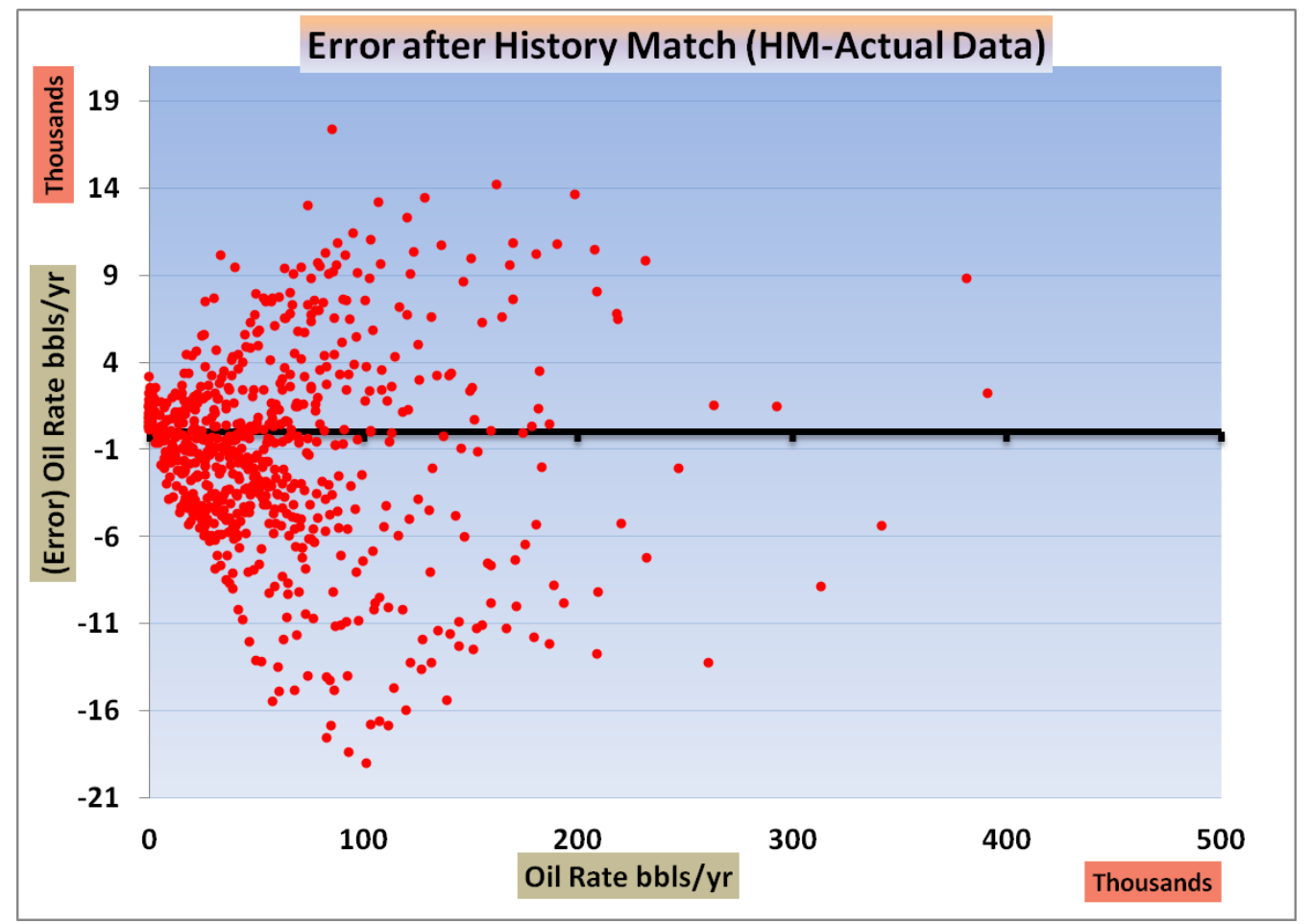

Figure 42- Error distribution for history matched results

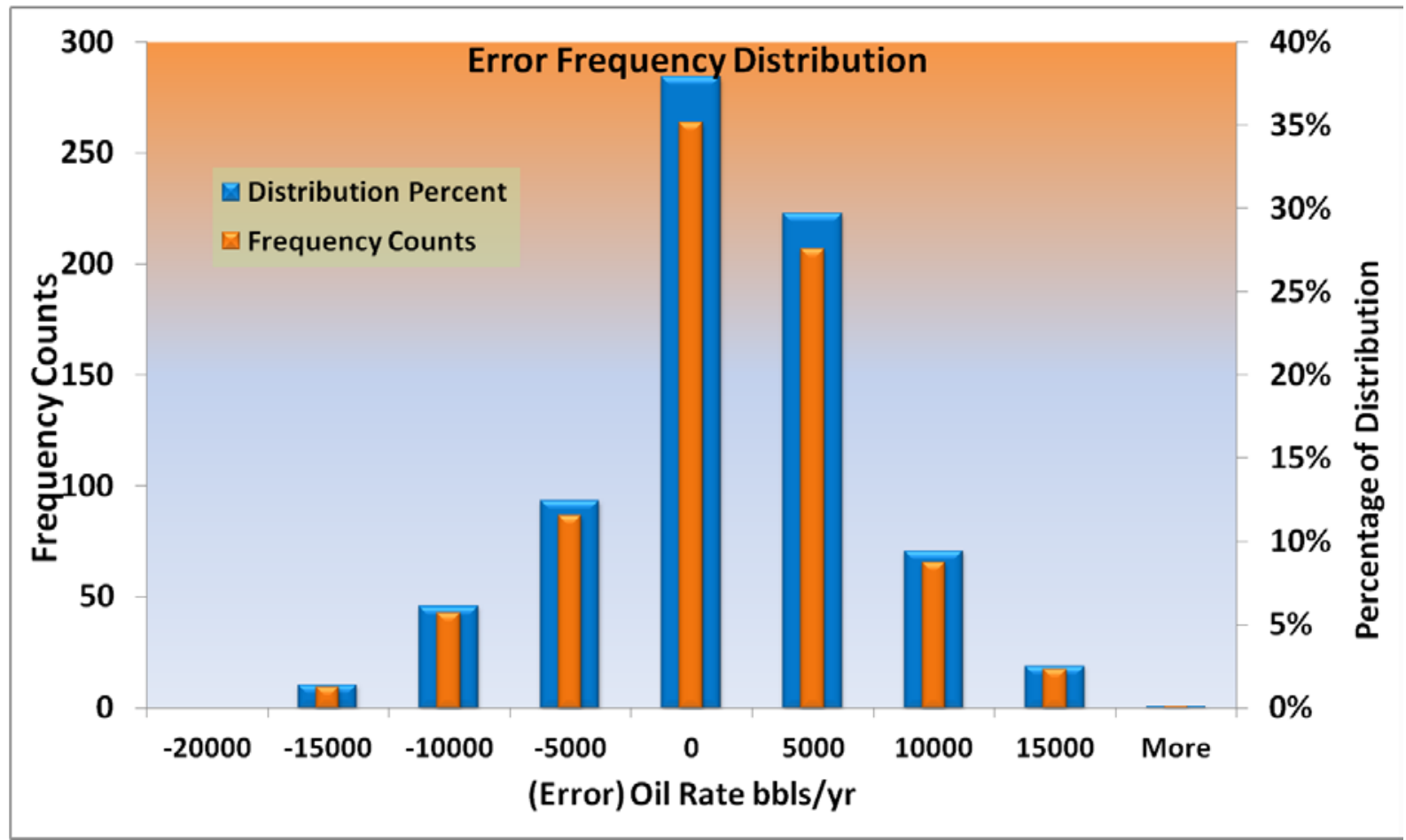

Figure 43- Error frequency distribution for the history match results 


\subsection{Summary and Conclusion}

Reservoir simulation and modeling is utilized throughout field development in different capacities. Sensitivity analysis, history matching, operations optimization and uncertainty assessment are the conventional analyses in a full field model study. Realistic modeling of the complexities of a reservoir requires a large number of grid blocks. As the complexity of a reservoir increases, and consequently the number of grid blocks increase, so does the time required to accomplish the abovementioned tasks. A relatively new technology known as Surrogate Reservoir Model has been introduced as a tool to address many time-consuming operations performed with reservoir simulation models. SRMs are replicas of full field models that run in fractions of a second. In this study, we tried to prove that SRM is an efficient tool to replicate the reservoir simulator performance faster and with a reasonable accuracy in order to assist the history match process.

History matching techniques are used in reservoir modeling to fine-tune reservoir properties such as porosity and permeability by matching the measured production data (pressure and production profiles at each well). The objective of this study was to prove and examine the utility of SRM as a tool for assisted history matching.

In this project, an SRM was created for a synthetic but heterogeneous and under-saturated oil field, with 24 production wells and 30 years of production history. The aim of the project was to achieve a match for the production history by tuning the static data (permeability distribution). SRM was trained using several heterogeneity realizations and was validated by a blind simulation run. Finally, the full field model was substituted by the trained SRM for the history match process.

Accomplishment of the objectives of this project included the following steps:

1. Development of a reservoir model using a commercial simulator. This model included about 5000 grid blocks with 24 production wells with 30 years of oil production history. This reservoir was under-saturated. A base-case permeability distribution was provided to initiate the modeling process.

2. An SRM was trained, calibrated and validated using ten geological realizations of this reservoir. The SRM was further validated using a complete blind realization of the reservoir. 
3. The SRM was used as the replica of a reservoir simulator (CMG-Builder ${ }^{\mathrm{TM}}$ ) in an assisted history match process. The oil production of all 24 wells was history matched in a short period of time by modifying the permeability distribution throughout the reservoir.

4. The final result of the history match was a permeability distribution that was compared with the original permeability distribution used as an input in the full filed model. The comparison is shown in Figure 37.

As previously mentioned, this work intended to prove the potential of an SRM to assist the history match process. Therefore, future studies of this work certainly are our interest. Studying more complex reservoirs such as reservoirs with faults, multi-phase reservoirs, reservoirs in saturated conditions, and the reservoirs with more wells and complex operational constraints, etc could be considered the future work of this study. In addition, the study could be extended by having multiple objective functions and combining SRM with the optimization methods (using the SRM for stochastic models). 


\section{References}

Ahmed, T., Link, C., Porter, K., Wideman, C., Himmer, P., \& Braun, J. (1997). Application of Neural Network Parameter Prediction in Reservoir Characterization and Simulation - A Case History: The Rabbit Hills Field. Latin American and Caribbean Petroleum Engineering Conference. Rio de Janeiro, Brazil.

Ali, J. K. (1994). Neural Networks: A New Tool for the Petroleum Industry? European Petroleum Computer Conference. Aberdeen, United Kingdom: Society of Petroleum Engineers,.

Al-Kaabi, A. U., \& Lee, W. J. (1990). An Artificial Neural Network Approach To Identify the Well Test Interpretation Model: Applications. SPE Annual Technical Conference and Exhibition. New Orleans, Louisiana: Society of Petroleum Engineers.

Amini, S., Mohaghegh, S. D., Gaskari, R., \& Bromhal, G. (2012). Uncertainty Analysis of a CO2 Sequestration Project Using Surrogate Reservoir Modeling Technique. SPE Western Regional Meeting,. Bakersfield, California, USA: Society of Petroleum Engineers.

Aminzadeh, F., \& deGroot, P. (2005). A Neural Networks Based Seismic Object Detection Technique. SEG Annual Meeting. Houston, Texas.

Athichanagorn, S., \& Horne, R. N. (1995). Automatic Parameter Estimation From Well Test Data Using Artificial Neural Network. SPE Annual Technical Conference and Exhibition. Dallas, Texas: Society of Petroleum Engineers.

Baldwin, J. L., Otte, D. N., \& Whealtley, C. L. (1989). Computer Emulation of Human Mental Processes: Application of Neural Network Simulators to Problems in Well Log Interpretation. SPE Annual Technical Conference and Exhibition. San Antonio, Texas.

Briones, M. F., Rojas, G., Moreno, J., \& Martinez, E. R. (1994). Application of Neural Networks in the Prediction of Reservoir Hydrocarbon Mixture Composition From Production Data. SPE Annual Technical Conference and Exhibition. New Orleans, Louisiana: Society of Petroleum Engineers.

Bush, M., \& Carter, J. (1996). Application of a Modified Genetic Algorithm to Parameter Estimation in Petroleum Industry. Intelligent Engineering Systems through Artificial Neural Networks, volume 6, P 397.

Castellini, A. (2005). Quantifying Uncertainty in Production Forecast for Fields with Significant History: A West African Case Study. International Petroleum Technology Conference. Doha, Qatar. 
Chen, W., Gavalas, G., Seinfeld, J. H., \& Wasserman, M. L. (1974). A New Algorithm for Automatic History Matching. SPE-AIME 48th Annual Fall Meeting. Las Vegas, U.SA.: Society of Petroleum Engineers.

Chen, Z. (2007). Reservoir Simulation: Mathematical Techniques in Oil Recovery. Society for Industrial and Applied Math.

Cheng-Dang, Z., Wu, S.-L., Mo, Z. C., Zhu, D. H., \& Xu, Z. V. (1994). Direct Identification of Hydrocarbon From Well Logs: A Neural Network Interpretation Approach. Annual Technical Meeting. Calgary, Alberta: Petroleum Society of Canada.

CMG, C. M. (n.d.). Computer Modelling Group Manual.

Coats, K., Dempsey, J., \& Henderson, J. (1968). A New Technique for Determining Reservoir Description from Field Performance Data. 43rd SPE Annual Fall Meeting. Houston, Texas, U.S.A.

Computer Modelling Group Ltd. (2011). User's Guide CMOST Studio. Calgary, Calgary, Canada: Computer Modelling Group Ltd.

Cosentino, L. (2001). Integrated Reservoir Studies.

David, H. J. (1993). Seismic Attribute Calibration Using Neural Networks. SEG Annual Meeting. Washington, DC: Society of Exploration Geophysicists.

Erbas, D., \& Christie, M. (2007). Effect of Sampling Strategies on Prediction Uncertainty Estimation. SPE Reservoir Simulation Symposium. Houston, Texas, U.S.A.

Ershaghi, I., Li, X., Hassibi, M., \& Shikari, Y. (1993). A Robust Neural Network Model for Pattern Recognition of Pressure Transient Test Data. SPE Annual Technical Conference and Exhibition. Houston, Texas: Society of Petroleum Engineers.

Ertekin, T., Abou-Kassem, J. H., \& King, G. R. (2001). Basic Applied Reservoir Simulation. In T. Ertekin, J. H. Abou-Kassem, \& G. R. King, Basic Applied Reservoir Simulation. Richardson, Texas: Society of Petroleum Engineers.

Fanchi, J. R. (2006). Principles of Applied Reservoir Simulation. Publisher: Elsevier Science and Technology Books, Inc.

Gharbi, R., \& Elsharkawy, A. (1997). Neural Network Model for Estimating The PVT Properties of Middle East Crude Oils. Middle East Oil Show and Conference. Bahrain.

Guohua Gao, Gaoming Li and A. C. Reynolds. (2004). A Stochastic Optimization Algorithm for Automatic History Matching. Society of Petroleum Engineers .

Hagan, M. T., Demuth, H. B., \& Beale, M. H. (2002). Neural Network Design. Hagan Publishing. 
Hajizadeh, Y. (2010). Ants Can Do History Matching. SPE Annual Technical Conference and Exhibition. Florence, Italy: Society of Petroleum Engineers.

Hajizadeh, Y., Christie, M., \& Demyanov, V. (2009). Ant Colony Optimization for History Matching. SPE EUROPEC/EAGE Annual Conference and Exhibition. Amsterdam, The Netherlands: Society of Petroleum Engineers.

Haykin, S. (1998). Neural Networks: A Comprehensive Foundation. Prentice Hall.

He, N., \& Chambers, K. (1999). Calibrate Flow Simulation Models With Well-Test Data to Improve History Matching. SPE Annual Technical Conference and Exhibition.

He, N., Reynolds, A., \& Oliver, D. (1997). Three-Dimensional Reservoir Description From Multiwell Pressure Data and Prior Information. SPE , 413.

Intelligent Solutions Inc. (2012). Retrieved 2011, from Intelligent Solutions Inc.: www.intelligentsolutionsinc.com

Jacquard, P., \& Jain, C. (1965). Permeability Distribution From Field Pressure Data. SPE , 281.

Jahns, H. (1966). A Rapid Method for Obtaining a Two-Dimensional Reservoir Description From Well Pressure Response Data. SPE , 315.

Jong-Se, L., \& Jungwhan, K. (2004). Reservoir Porosity and Permeability Estimation from Well Logs using Fuzzy Logic and Neural Networks. SPE Asia Pacific Oil and Gas Conference and Exhibition. Perth, Australia.

Kabir, C., Chien, M., \& Landa, J. (2003). Experiences with automated history matching. SPE . Kalam, M., Al-Alawi, S., \& Al-Mukheini, M. (1996). Assessment of Formation Damage Using Artificial Neural Networks. SPE Formation Damage Control Symposium. Lafayette, Louisiana.

Kathrada, M. (2009). Uncertainty Evaluation of Reservoir Simulation Models using Particle Swarms and Hierarchical Clustering. Edinburgh: PhD thesis, Heriot Watt University.

Key, S., Nielsen, H., Signer, C., Sønneland, L., Waagb ø, K., \& H. Veire, H. (1997). Fault And Fracture Classification Using Artifical Neural Networks - Case Study From the Ekofisk Field. SEG Annual Meeting. Dallas, Texas.

Krose, B., \& Smagt, P. v. (1996). An introduction to neural networks. Amsterdam, The netherlands: The University of Amsterdam.

Kruger, W. D. (1961). Determining Areal Permeability Distribution by Calculations. J. Pet. Tech. 691 .

L.Thomas, A., \& Pointe, P. R. (1995). Conductive fracture identification using neural networks. The 35th U.S. Symposium on Rock Mechanics (USRMS). Reno, NV. 
Landa, J. e. (2000). Reservoir Characterization Constrained to Well Test Data: A Field Example. SPEREE .

Mantica, S. C. (2002). Combining Global and Local Optimization Techniques for Automatic History Matching Production and Seismic Data. SPE Journal , 123-130.

Maschio, C., \& Schiozer, D. (2005). Development and Application of Methodology for Assisted History Matching. Society of Petroleum Engineers .

Masoud, N. (1998). Neural Network Knowledge-Based Modeling of Rock Properties Based on Well Log Databases. SPE Western Regional Meeting. Bakersfield, California.

Mattax, C. C., \& Dalton, R. L. (1990). Reservoir Simulation. Richardson, TX: Society of Petroleum Engineer.

Maucec, M. D. (2007). Streamline-based history matching and uncertainty, Markov-chain Monte Carlo study of an offshore oil field. SPE Annual Technical Conference and Exhibition. Anaheim, California, U.S.A.

Mohaghegh, S. (2009). Artificial Intelligence and Data Mining: Enabling Technology for Smart Fields. SPE's The Way Ahead Journal , 14-19.

Mohaghegh, S. D. (1995). Neural Network: What It Can Do for Petroleum Engineers. Journal of Petroleum Technology .

Mohaghegh, S. D. (2006). Quantifying Uncertainties Associated With Reservoir Simulation Studies Using a Surrogate Reservoir Model. SPE Annual Technical Conference and Exhibition. San Antonio, Texas, USA: Society of Petroleum Engineers.

Mohaghegh, S. D. (2011). Reservoir Simulation and Modeling Based on Pattern Recognition. SPE Digital Energy Conference and Exhibition. Woodlands, Texas.

Mohaghegh, S. D. (2000). Virtual-Intelligence Applications in Petroleum Engineering: Part 1Artificial Neural Networks. Journal of Petroleum Technology .

Mohaghegh, S. D., Amini, S., Gholami, V., Gaskari, R., \& Bromhal, G. (2012). Grid-Based Surrogate Reservoir Modeling (SRM) for Fast Track Analysis of Numerical Reservoir Simulation Models at the Gridblock Level. SPE Western Regional Meeting. Bakersfield, California, USA: Society of Petroleum Engineers.

Mohaghegh, S. D., Hafez, H., Gaskari, R., Hajizadeh, M., \& Kenawy, M. (2006). Uncertainty Analysis of a Giant Oil Field in the Middle East Using Surrogate Reservoir Model. Abu Dhabi International Petroleum Exhibition and Conference. Abu Dhabi, UAE: Society of Petroleum Engineers.

Mohaghegh, S. D., Liu, J., Gaskari, R., Maysami, M., \& Olukoko, O. A. (2012a). Application of Surrogate Reservoir Models (SRM) to an Onshore Green Field in Saudi Arabia; Case Study. 
North Africa Technical Conference and Exhibition. Cairo, Egypt: Society of Petroleum Engineers.

Mohaghegh, S. D., Liu, J., Gaskari, R., Maysami, M., \& Olukoko, O. (2012b). Application of Well-Base Surrogate Reservoir Models (SRMs) to Two Offshore Fields in Saudi Arabia, Case Study. SPE Western Regional Meeting. Bakersfield, California, USA: Society of Petroleum Engineers.

Mohaghegh, S. D., Platon, V., \& Ameri, S. (1998). Candidate Selection for Stimulation of Gas Storage Wells Using Available Data With Neural Networks and Genetic Algorithms. SPE Eastern Regional Meeting. Pittsburgh, Pennsylvania.

Mohaghegh, S. (2010). Surrogate Reservoir Model. European Geological Union General Assembly. EGU 2010. Vienna, Austria.

Mohaghegh, S., Modavi, A., Hafez, H., \& Haajizadeh, M. (2009). Development of Surrogate Reservoir Model (SRM) for Fast Track Analysis of a Complex Reservoir. International Journal of Oil, Gas and Coal Technology , 2-23.

Mohaghegh., S., Arefi, R., Ameri., S., \& Rose., D. (1995). Design and Development of An Artificial Neural Network for Estimation of Formation Permeability. SPE Computer Applications .

Mohamed, L., Christie, M., \& Demyanov, V. (2009). Comparison of Stochastic Sampling Algorithms for Uncertainty Quantification. Reservoir Simulation Symposium. Woodlands, Texas, U.S.A.

Nikravesh, M., Kovscek, A., Johnston, R., \& Patzek, T. (1996). Prediction of Formation Damage During Fluid Injection into Fractured, Low Permeability Reservoirs via Neural Networks. SPE Formation Damage Control Symposium. Lafayette, Louisiana: Society of Petroleum Engineers.

Oloso, M. A., Khoukhi, A., Abdulraheem, A., \& Elshafei, M. (2009). Prediction of Crude Oil Viscosity and Gas/Oil Ratio Curves Using Recent Advances to Neural Networks. SPE/EAGE Reservoir Characterization and Simulation Conference. Abu Dhabi, UAE.

Osman, E. A., Abdel-Wahhab, O. A., \& Al-Marhoun, M. A. (2001). Prediction of Oil PVT Properties Using Neural Networks. SPE Middle East Oil Show. Bahrain.

Ouenes, A., Brefort, B., Meunier, G., Dupere, S., \& Intragaz. (1993). A New Algorithm for Automatic History Matching: Application of Simulated Annealing Method (SAM) to Reservoir Inverse Modeling. Society of Petroleum Engineers .

Palatnic, B., Zakirov, L., Haugen, S., \& van Roosmalen, J. (1993). New Approaches to Multiple History Matching. Seventh European Symposium on Improved Oil Recovery. Moscow. 
Rodriguez, A. A., Klie, H., Wheeler, M. F., \& Banchs, R. (2007). Assessing Multiple Resolution Scales in History Matching With Metamodels. SPE Reservoir Simulation Symposium. Houston, Texas, U.S.A.

Roth, G., \& Tarantoia, A. (1992). Inversion of Seismic Waveforms Using Neural Networks. SEG Annual Meeting. New Orleans, Louisiana: Society of Exploration Geophysicists.

Sadiq, T., \& I.S. Nashawi. (2000). Using Neural Networks for Prediction of Formation Fracture Gradie. SPE/CIM International Conference on Horizontal Well Technology. Calgary, Alberta, Canada.

Sampaio, T., Filho, V. M., \& Neto, A. d. (2009). An Application of Feed Forward Neural Network as Nonlinear Proxies for Use During the History Matching Phase. Latin American and Caribbean Petroleum Engineering Conference. Cartagena de Indias, Colombia.

Silva, P. C., Maschio, C., \& Schiozer, D. J. (2008). Application of Neural Network and Global Optimization in History Matching. Journal of Canadian Petroleum Technology .

Silva, P. C., Maschio, C., \& Schiozer, D. J. (2006). Applications of the Soft Computing in the Automated History Matching. Canadian International Petroleum Conference. Calgary, Alberta: Petroleum Society of Canada.

Singh, V., Painuly, P. K., Srivastava, A. K., Tiwary, D. N., \& Chandra, M. (2008). Neural Networks And Their Applications In Lithostratigraphic Interpretation of Seismic Data For Reservoir Characterization. World Petroleum Congress. Madrid, Spain.

Slater, G., \& Durrer, E. (1970). Adjustment of Reservoir Simulation Models to Match Field Performance. SPE 45th Annul Fall Meeting. Houston, Texas, U.S.A.

Sousa, S. (2007). Scatter search metaheuristic applied to the history matching problem. SPE Annual Technical Conference and Exhibition. Anaheim, California, U.S.A.

Subbey, S., \& Christie, M. (2003). A Strategy for Rapid Quantification of Uncertainty in Reservoir Performance Prediction. SPE Reservoir Simulation Symposium. Houston, Texas, U.S.A.

Sultan, A., Ouenes, A., \& Weiss, W. (1994). Automatic History Matching for an Integrated Reservoir Description and Improving Oil Recovery. SPE Permian Sssin Oil and Gas Recovery Conference (pp. 741-748). Midland, Texas: Society of Petroleum Engineers.

Sultanp, M. A., \& Al-Kaabi, A. U. (2002). Application of Neural Network to the Determination of Well-Test Interpretation Model for Horizontal Wells. SPE Asia Pacific Oil and Gas Conference and Exhibition. Melbourne, Australia.

Toronyi, R., \& Saleri, N. (1988). Engineering control on reservoir engineering, Part 2. Society of Petroleum Engineers . 
Tyler, K., Svanes, T., \& Omdal, S. (1993). Faster History Matching and Uncertainty in Predicted Production Profiles With Stochastic Modeling. 68th Annual Technical Conference and Exhibition of the Society of Petroleum Engineers. Houston: Society of Petroleum Engineers.

Watts, J. (1997). Reservoir Simulation: Past, Present, and Future. SPE Computer Applications , 171-176.

Yang, C., Ngheim, L., \& Card, C. (2007). Reservoir Model Uncertainty Quantification through Computer-Assisted History Matching. SPE Annual Technical Conference and Exhibition.

Anaheim, California, U.S.A.

Yang, H., \& Huang, K.-Y. (1991). Hybrid Neural Network For Seismic Pattern Recognition. SEG Annual Meeting. Houston, Texas: Society of Exploration Geophysicists.

Zangl, G., Giovannoli, M., \& Stundner, M. (2006). Application of Artificial Intelligence in Gas Storage Management. SPE Europec/EAGE Annual Conference and Exhibition. Vienna, Austria. 


\section{Appendix A- Wells Historical Performance}

\section{a. Monthly bottom-hole pressure profile}
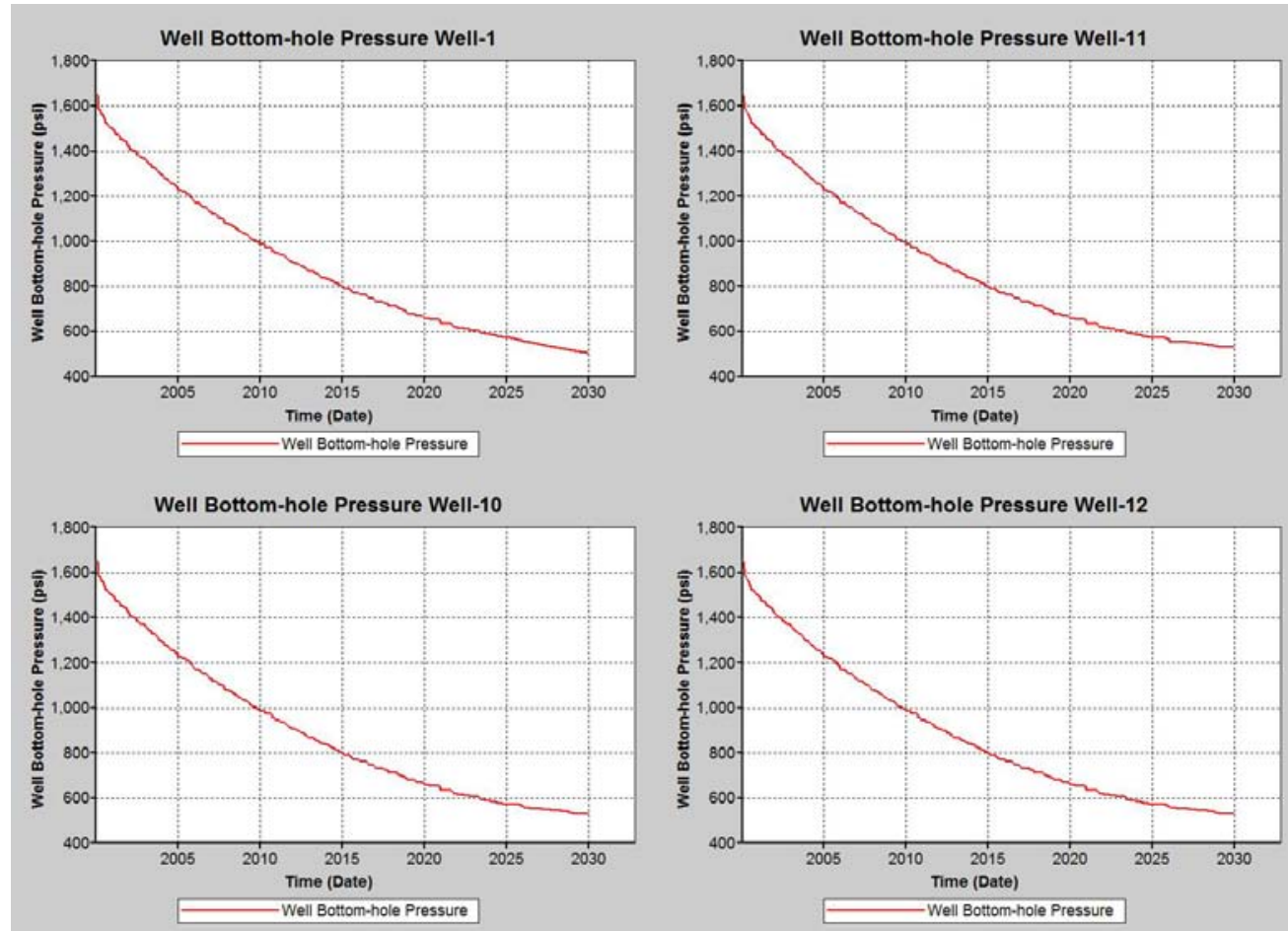

Figure 44- Bottom-hole pressure profile for the wells 1, 10, 11, and 12
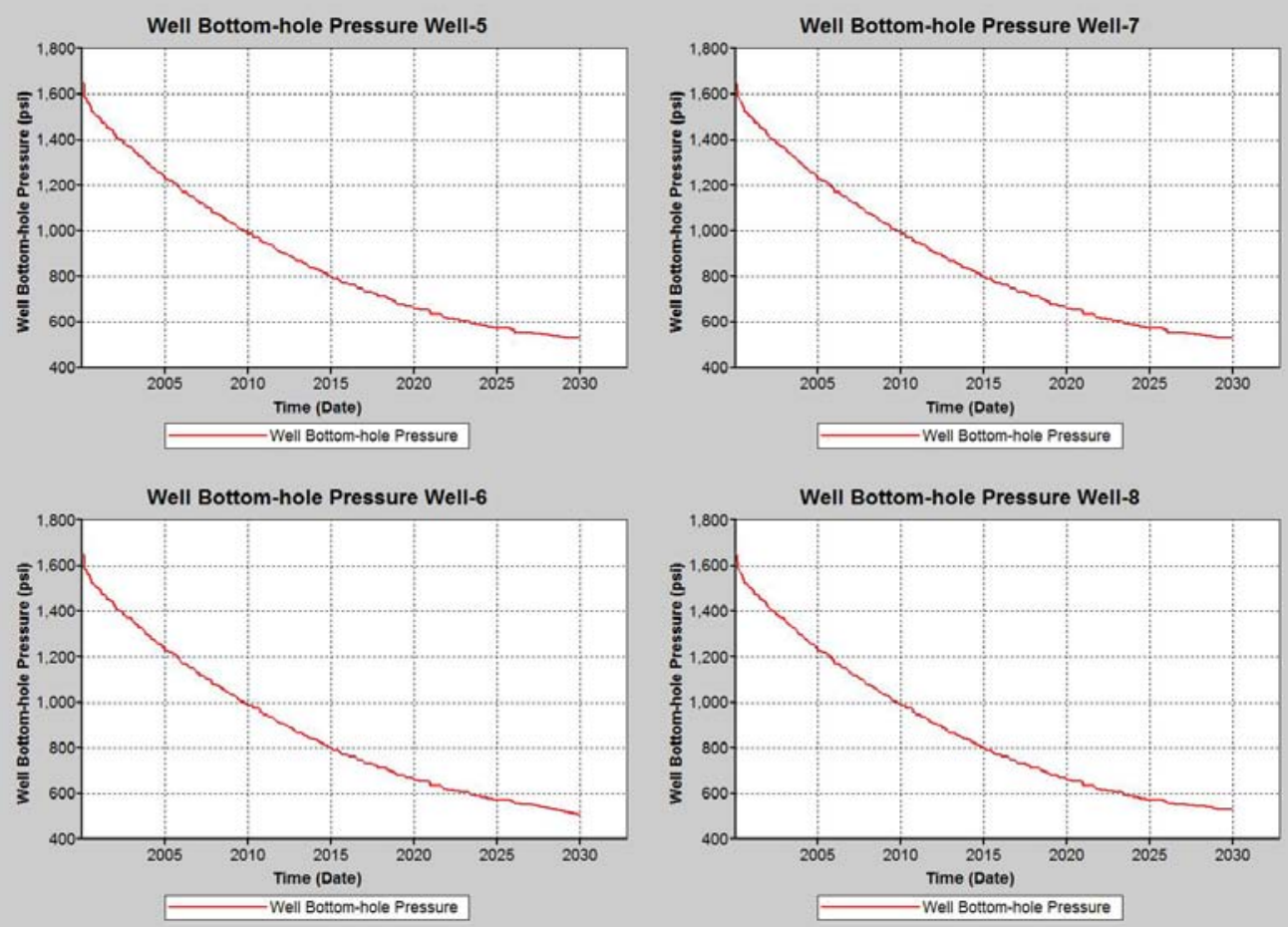

Figure 45- Bottom-hole pressure profile for the wells 5, 6, 7, and 8 

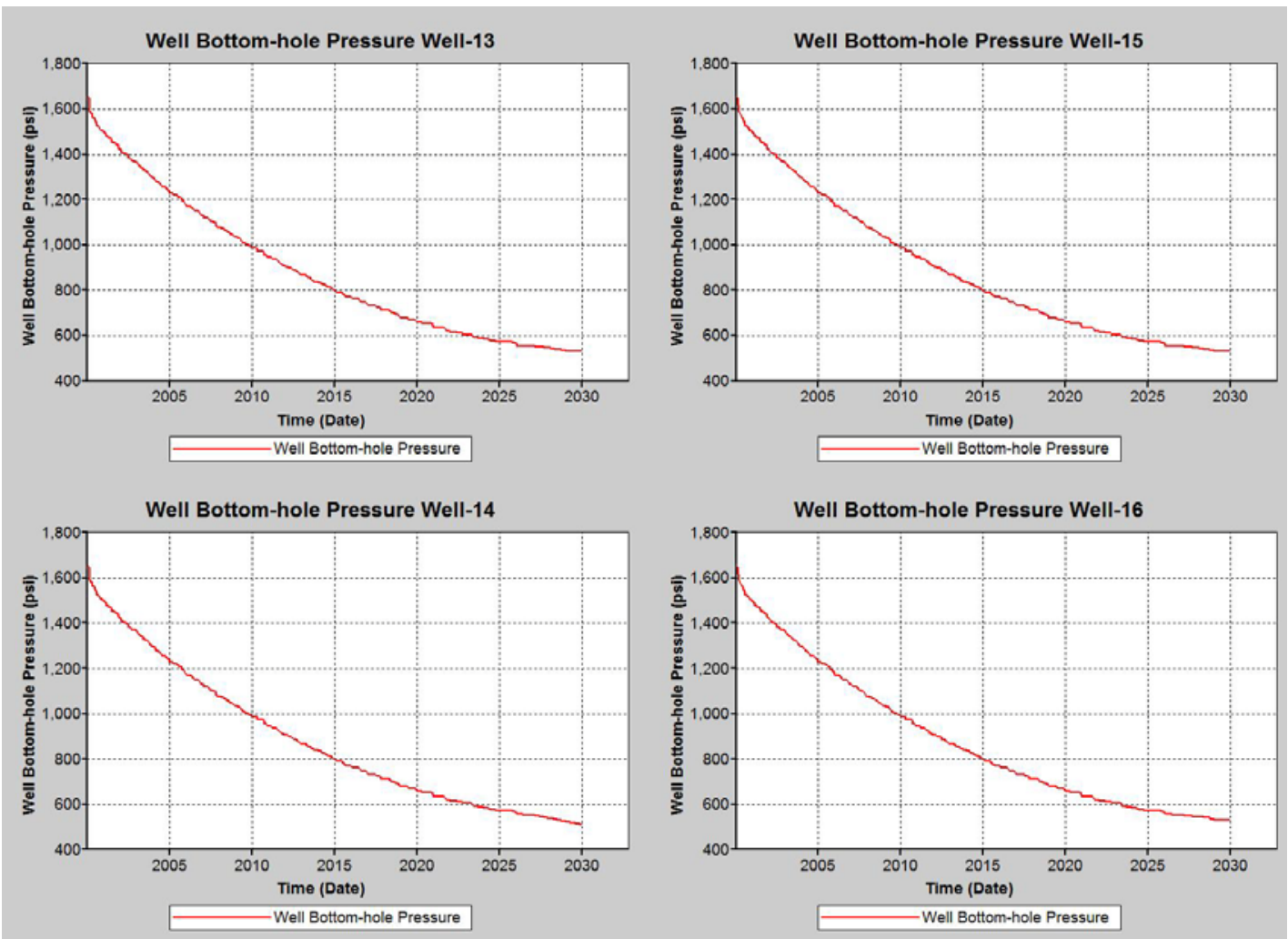

Figure 46-Bottom-hole pressure profile for the wells 13, 14, 15, and 16
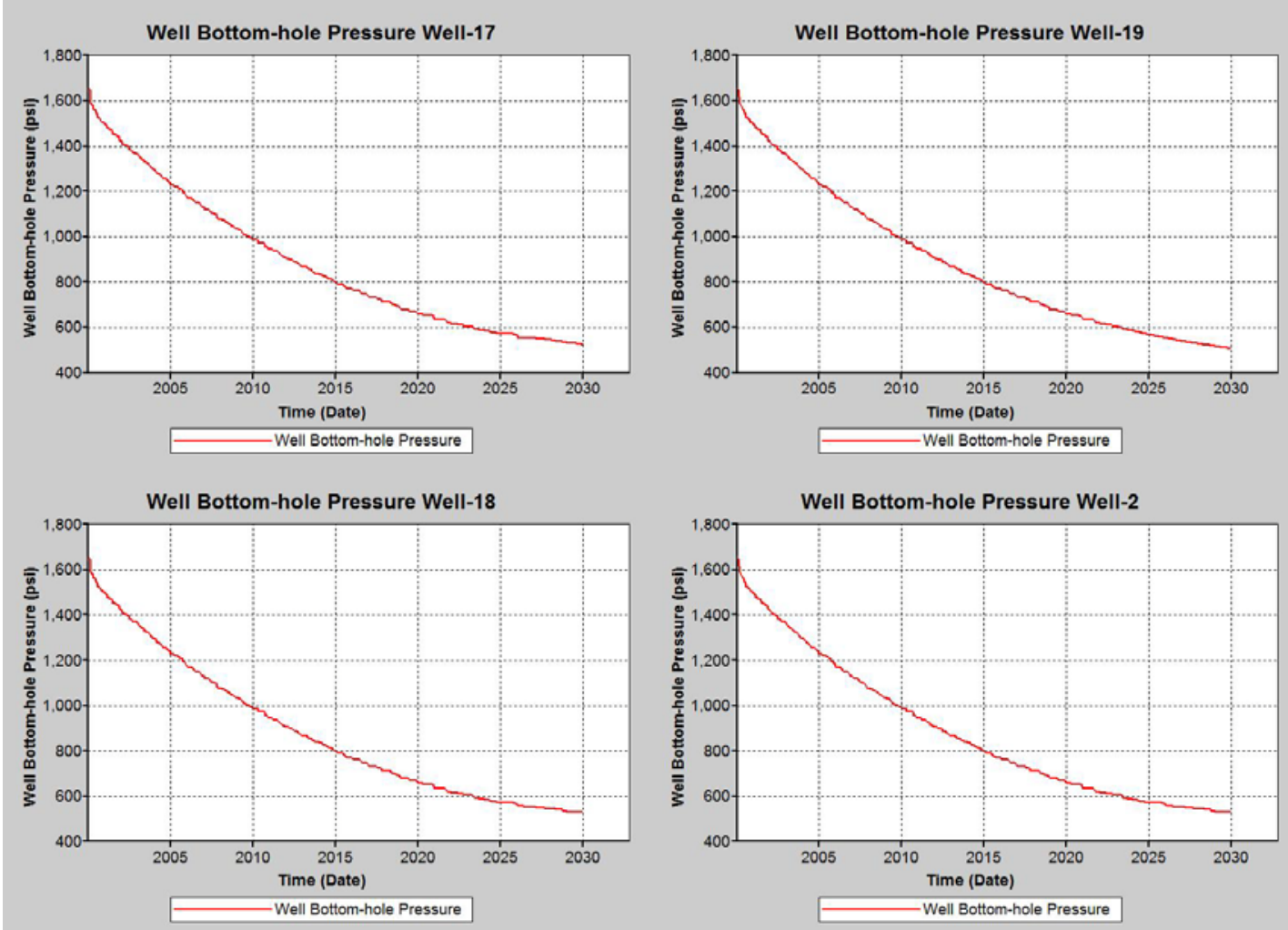

Figure 47-Bottom-hole pressure profile for the wells 17, 18, 19, and 2 

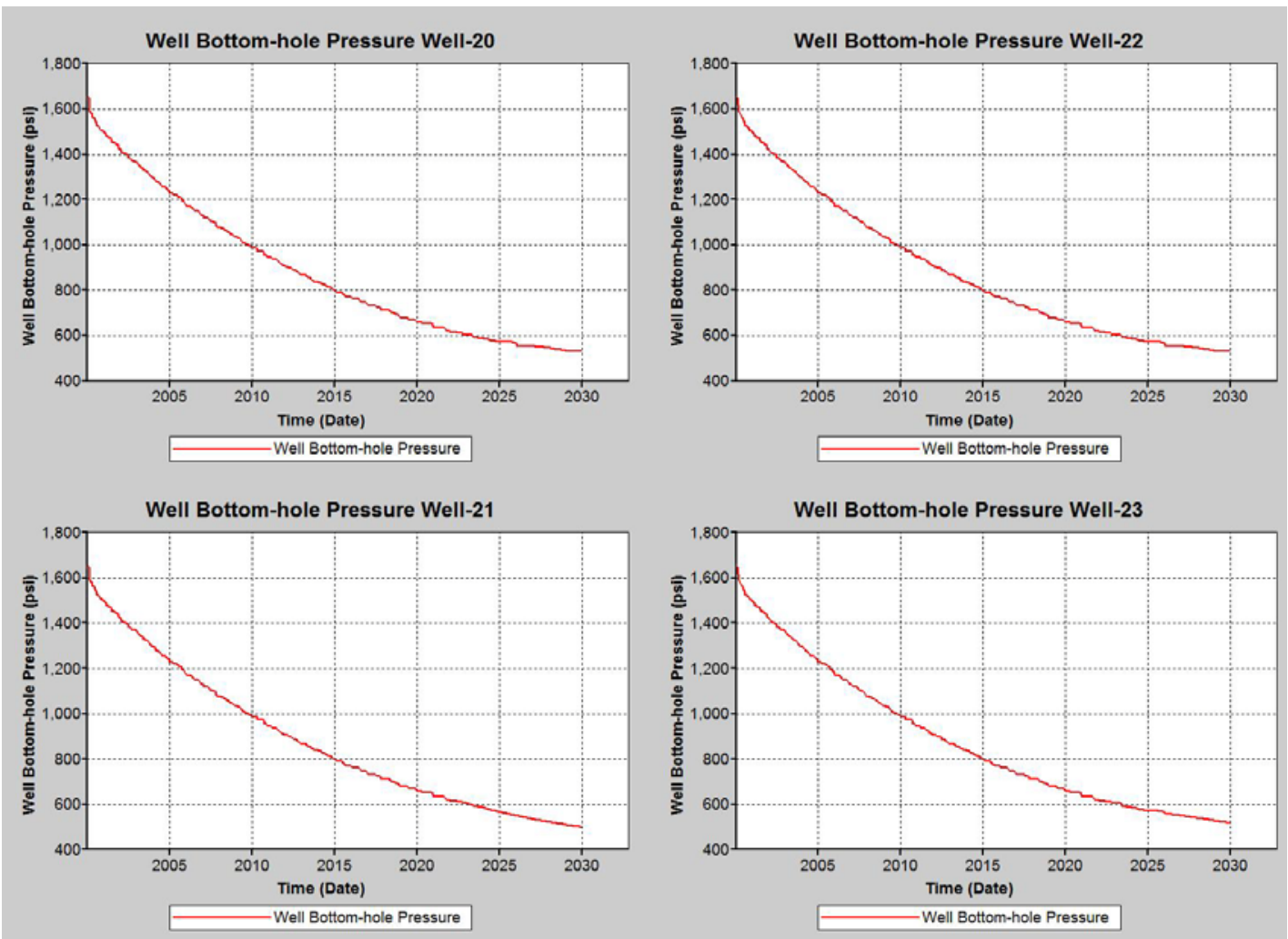

Figure 48- Bottom-hole pressure profile for the wells 20, 21, 22, and 23
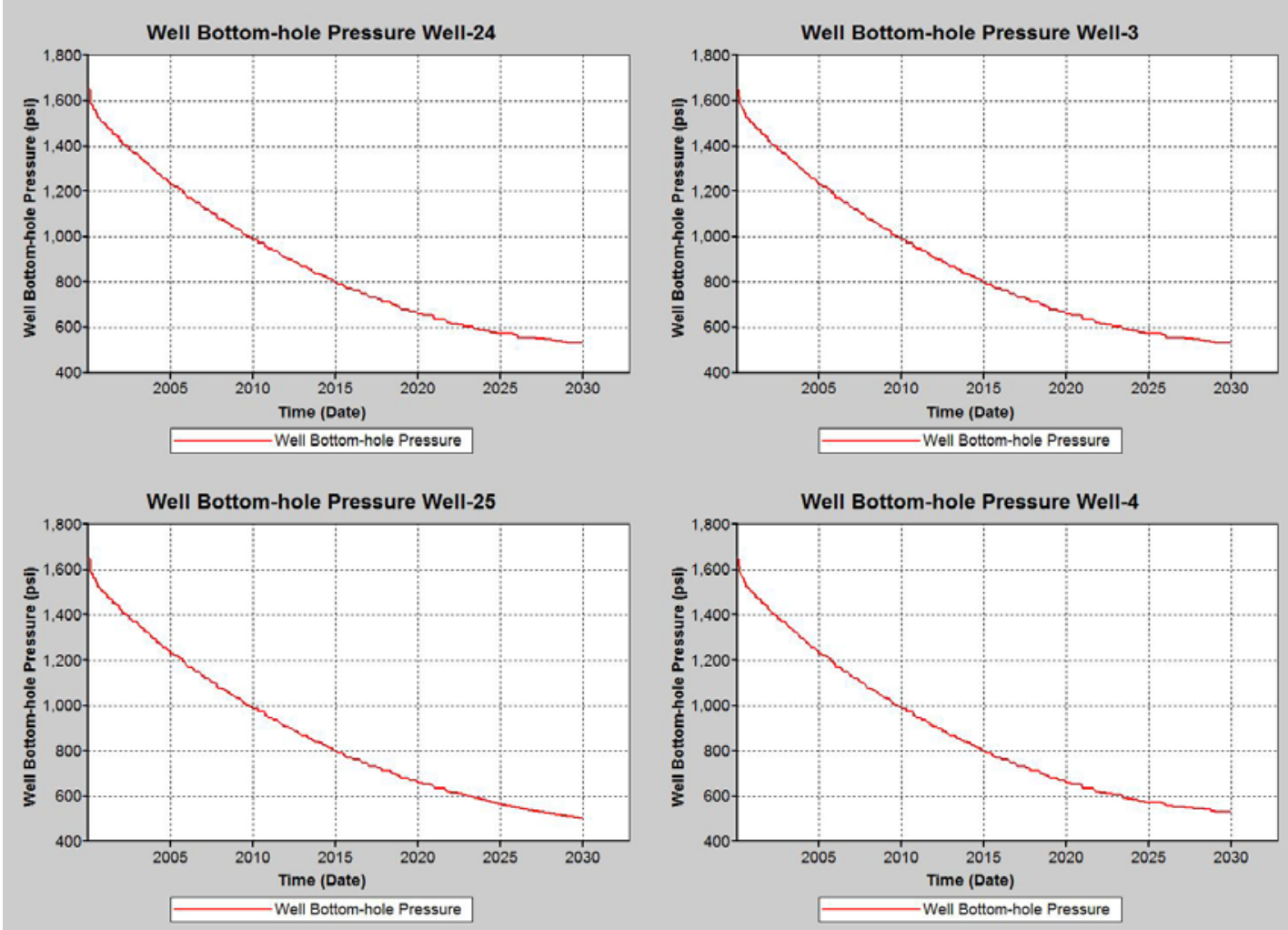

Figure 49- Bottom-hole pressure profile for the wells 3, 4, 24, and 25 


\section{b. Production historical performance}
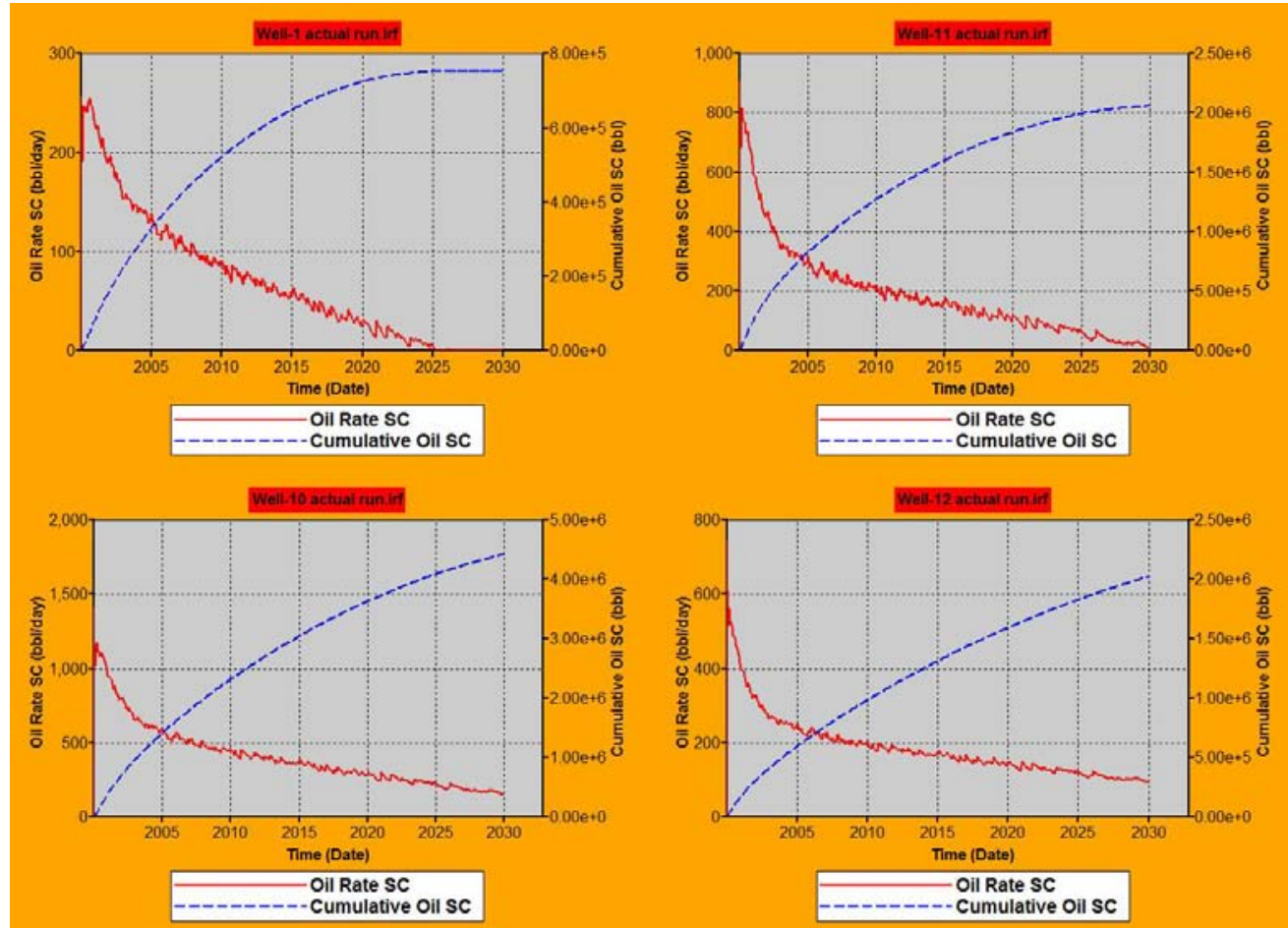

Figure 50- Oil rate and cumulative production for the wells 1, 10, 11 and 12
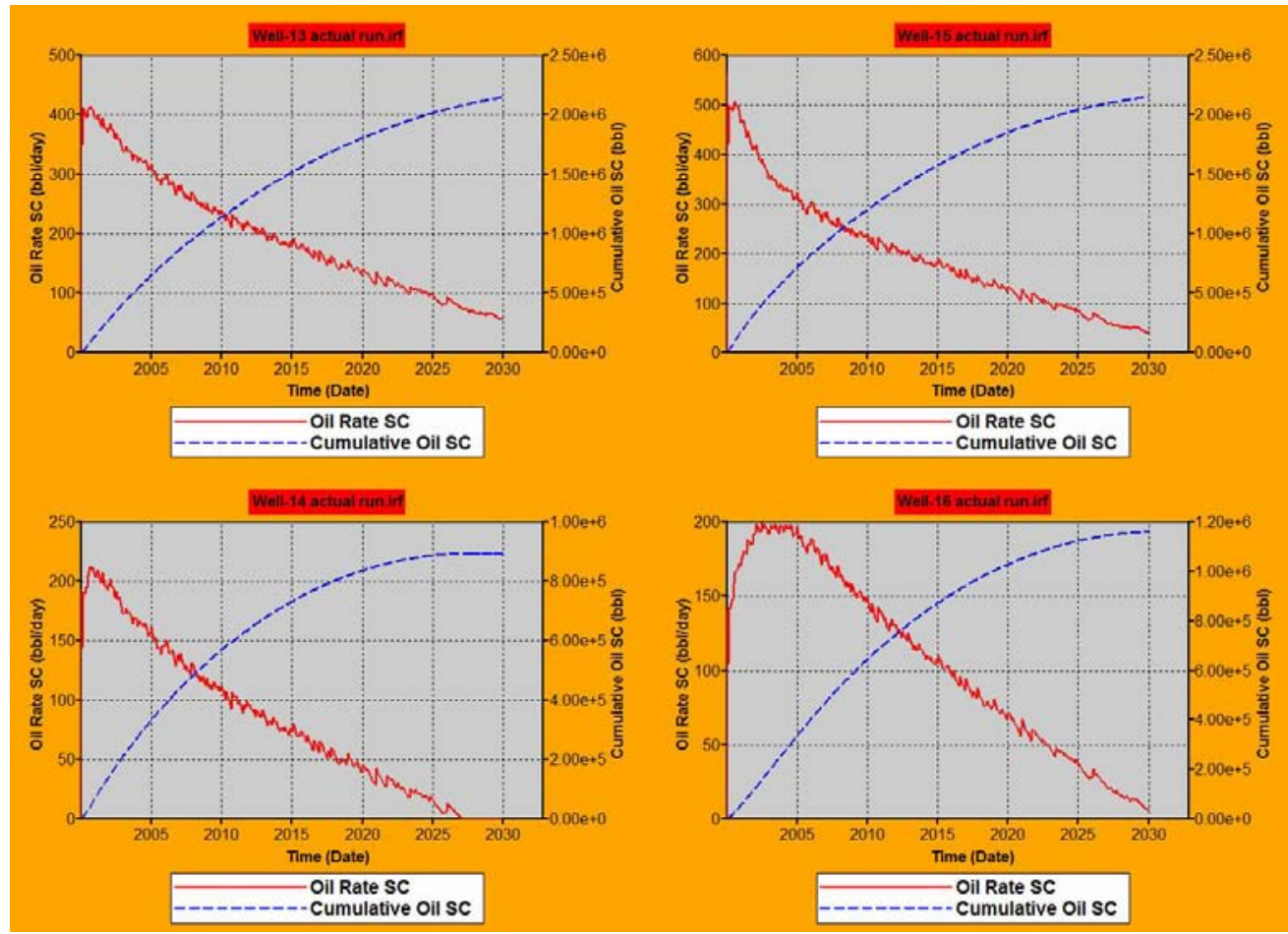

Figure 51- Oil rate and cumulative production for the wells $13,14,15$ and 16 

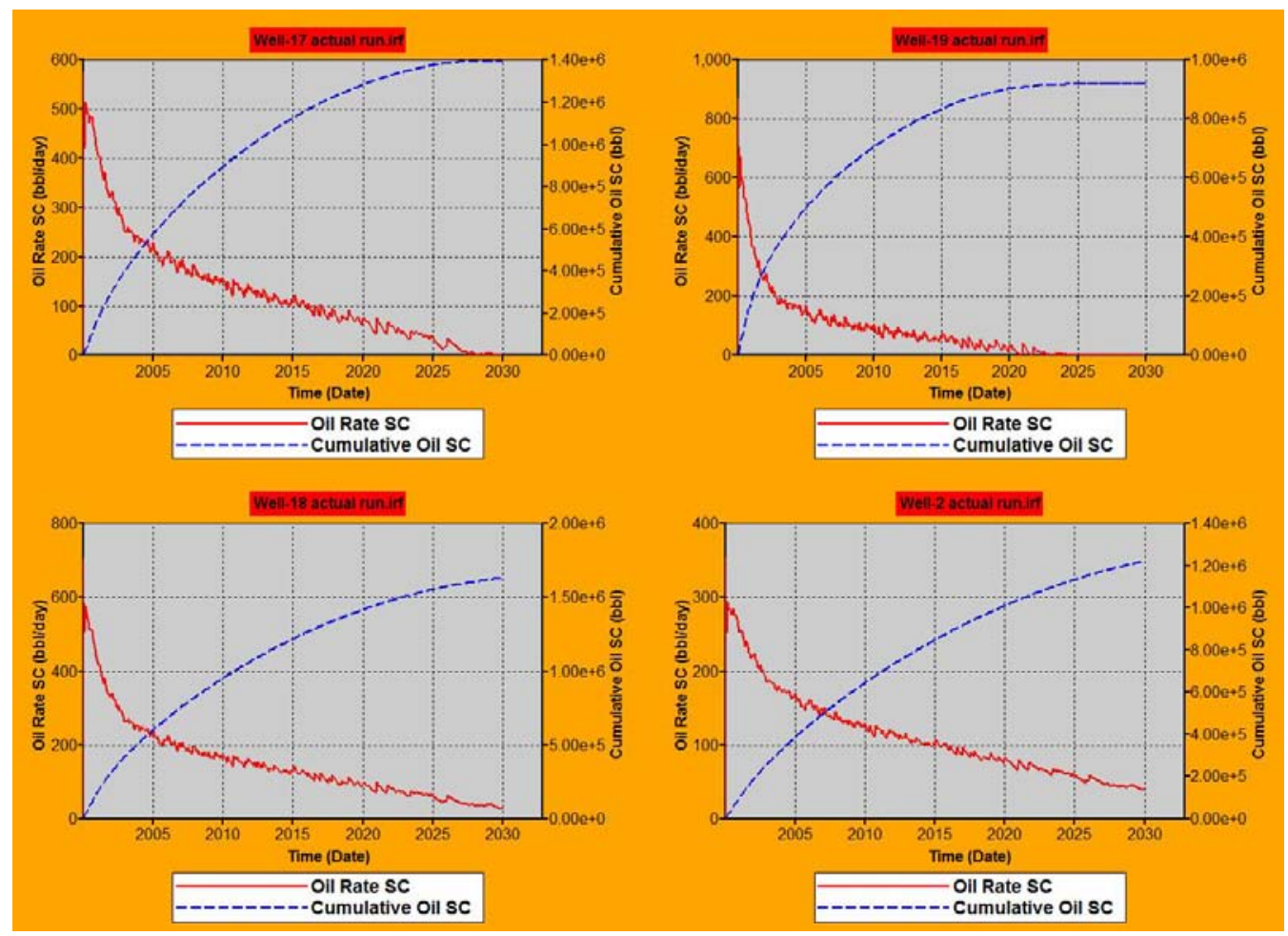

Figure 52- Oil rate and cumulative production for the wells 17, 18, 19 and 2

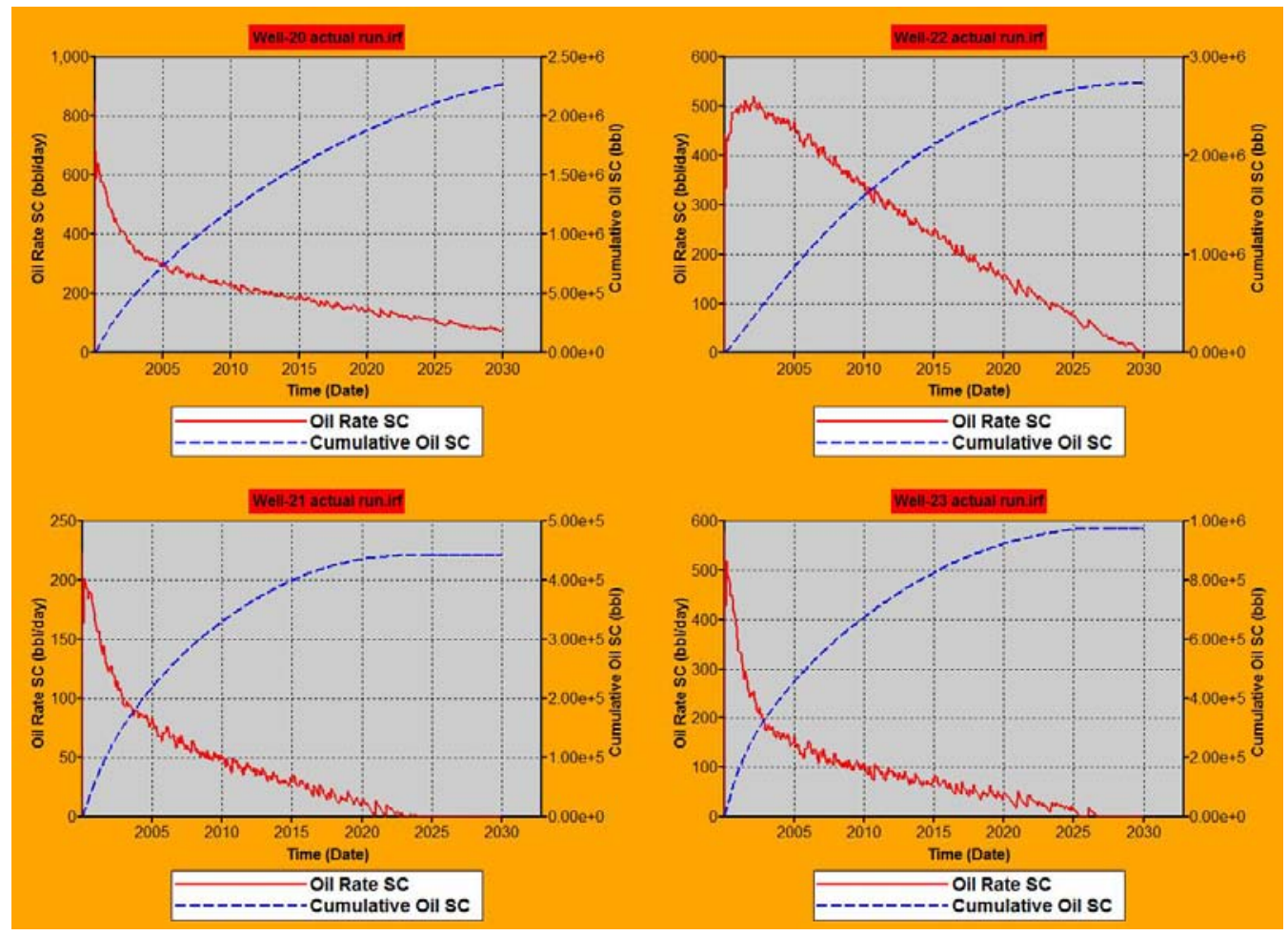

Figure 53- Oil rate and cumulative production for the wells 20, 21, 22 and 23 

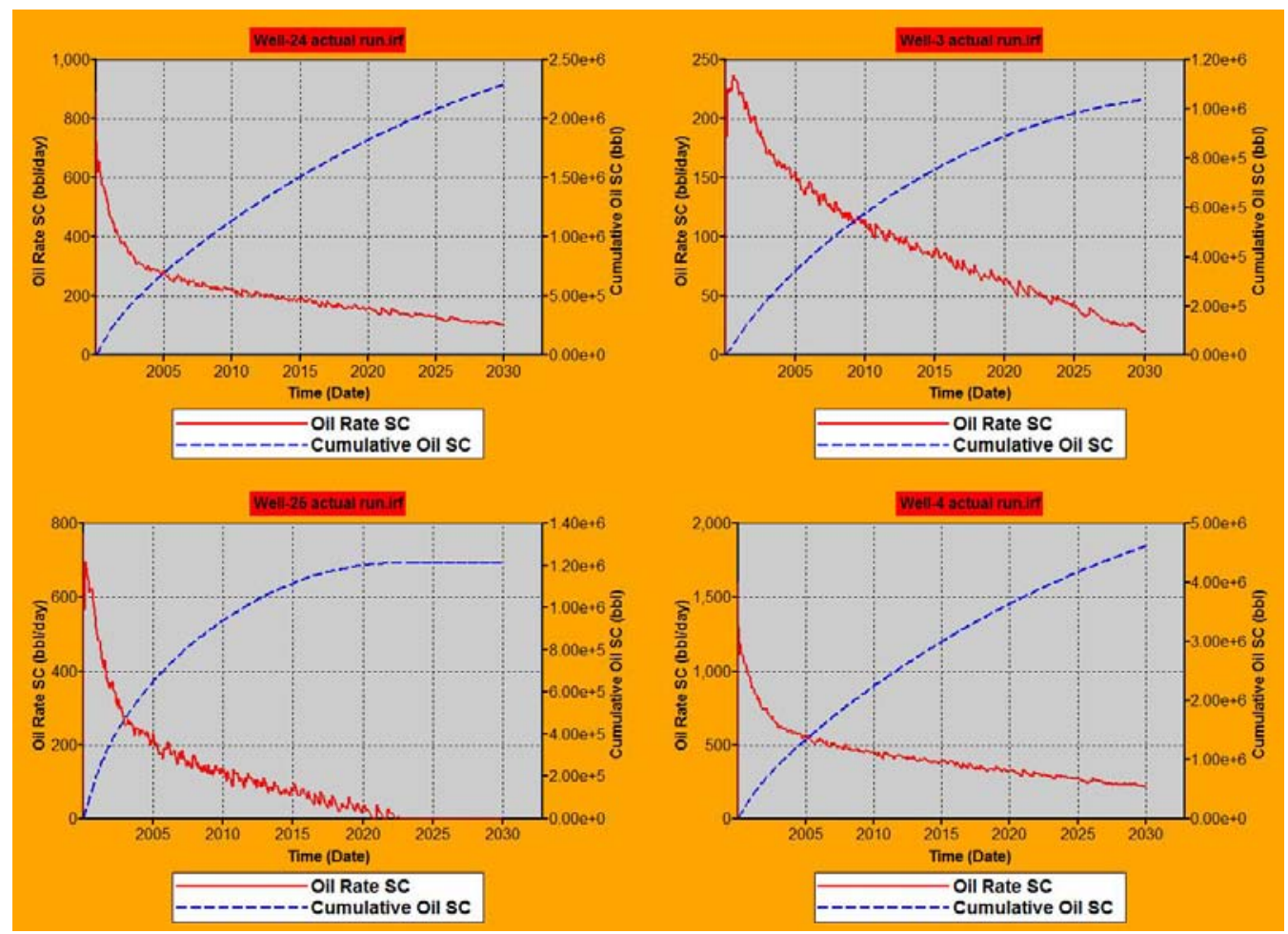

Figure 54- Oil rate and cumulative production for the wells 24, 25, 3 and 4

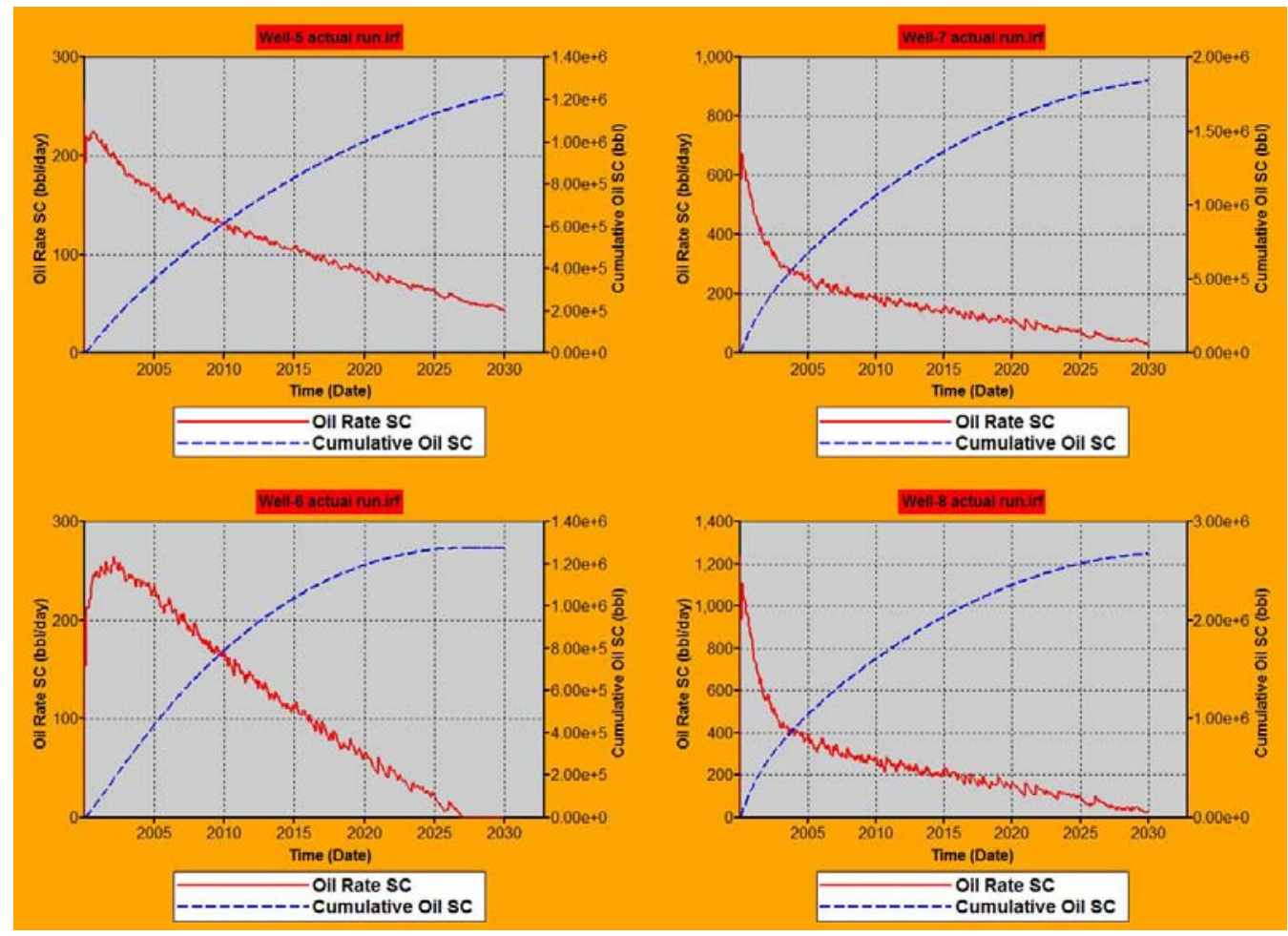

Figure 55- Oil rate and cumulative production for the wells 5, 6, 7 and 8 


\section{Appendix B-10 Created permeability distributions for training SRM}

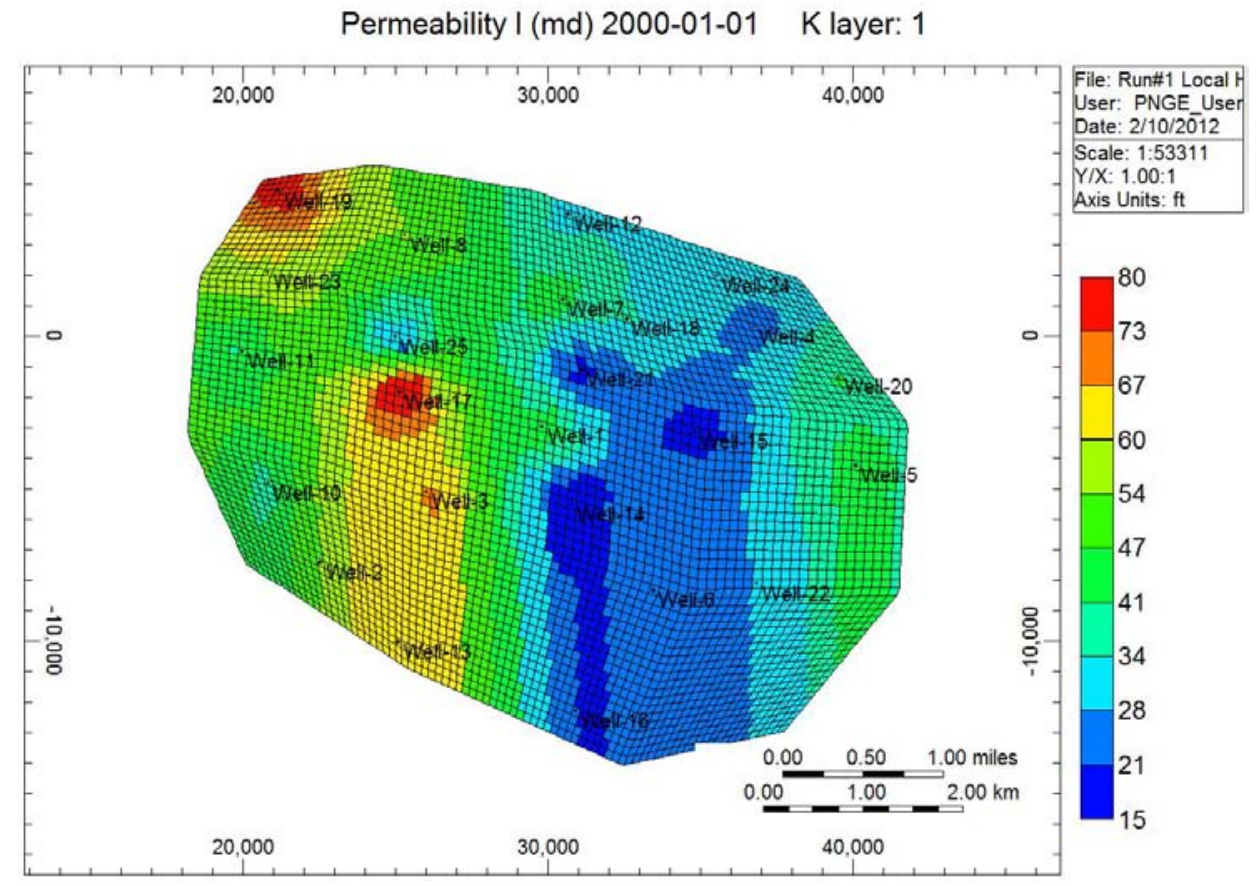

Figure 56- Permeability Map for different Training Realizations- Run\#1

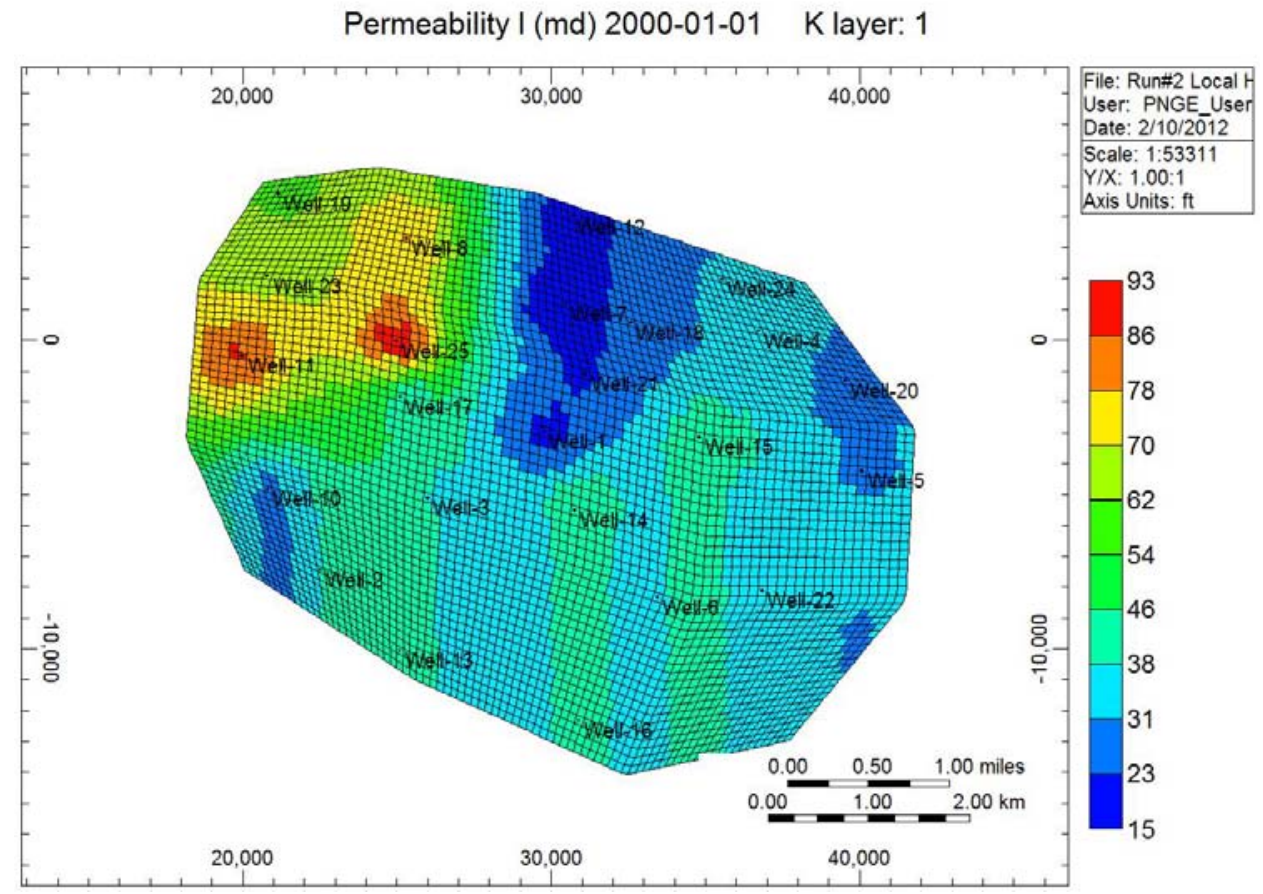

Figure 57- Permeability Map for different Training Realizations- Run\#2 
Permeability I (md) 2000-01-01 K layer: 1

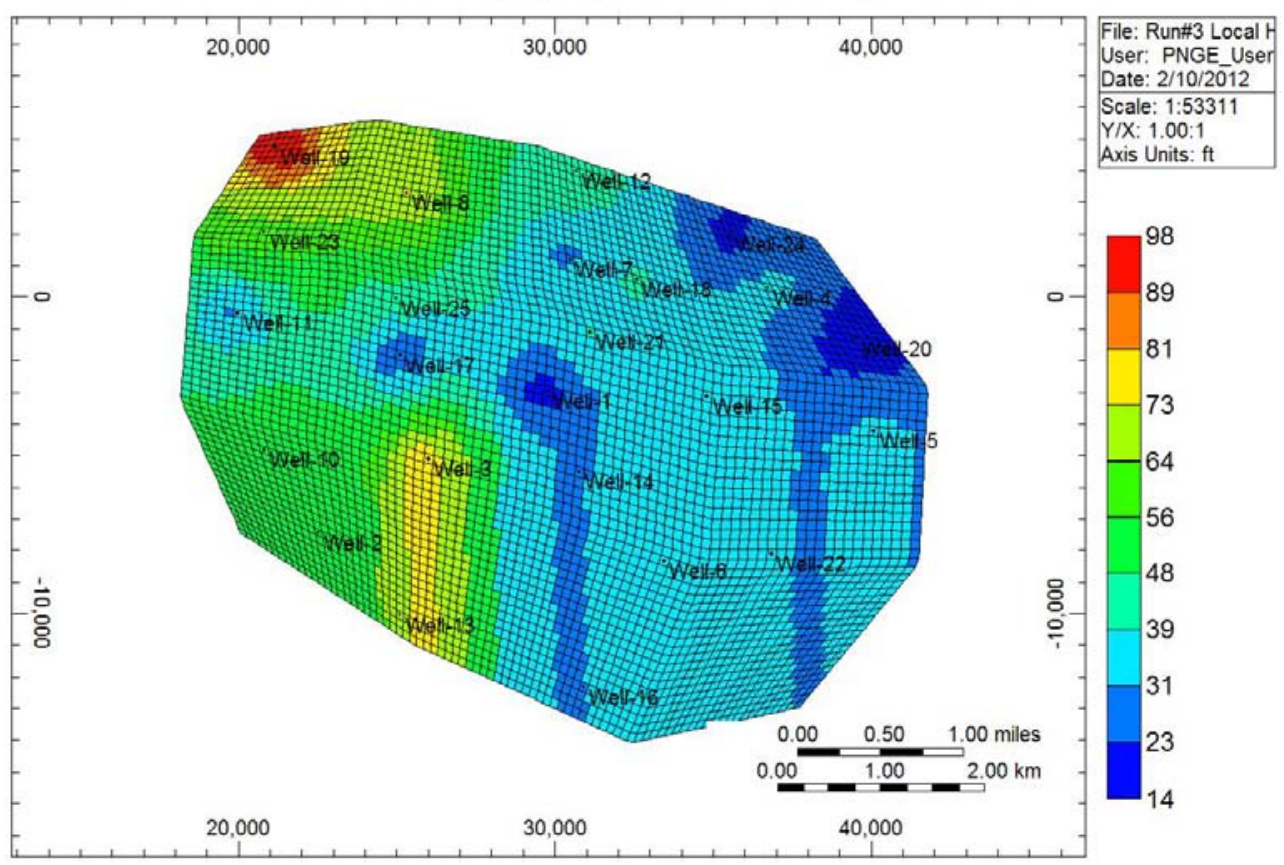

Figure 58- Permeability Map for different Training Realizations- Run\#3

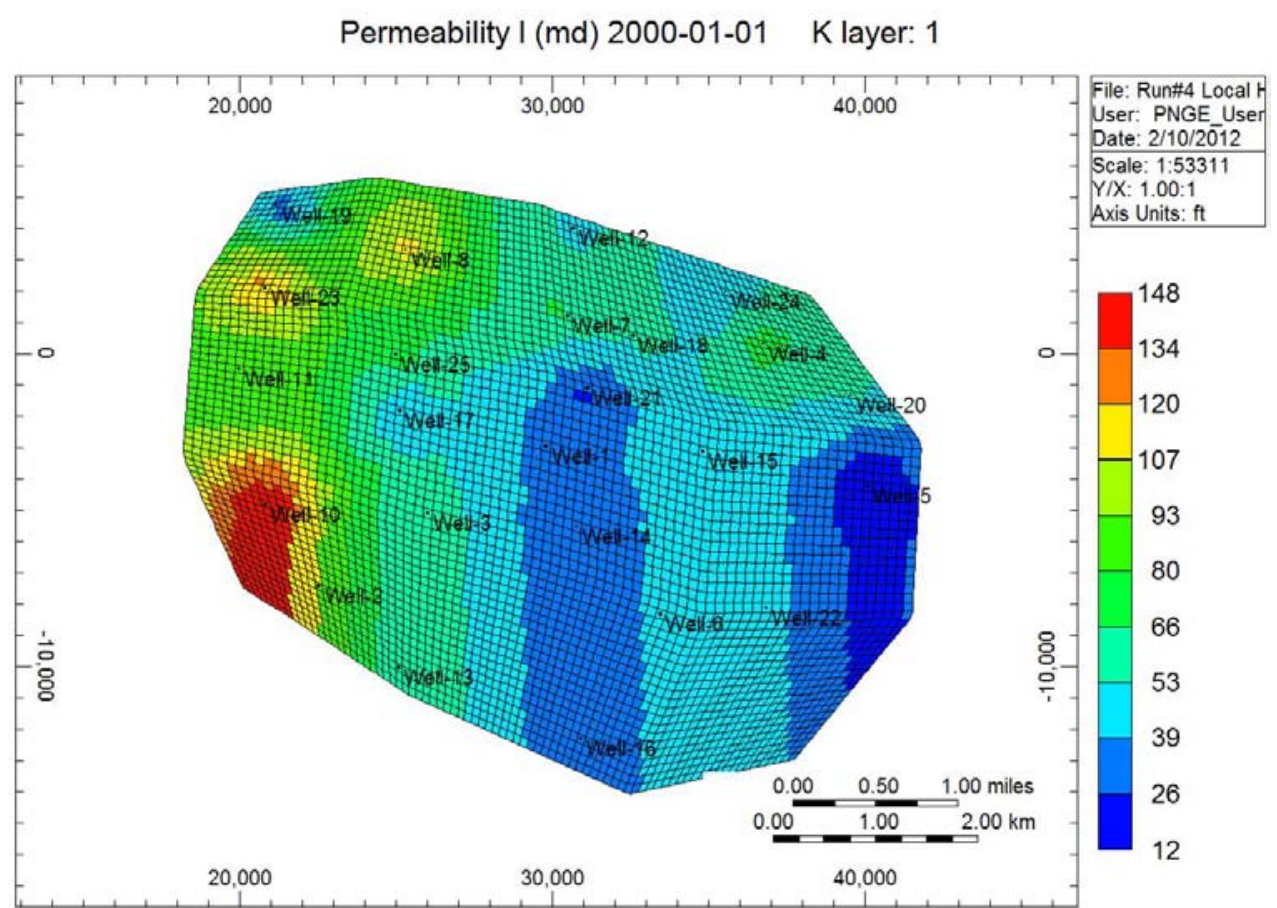

Figure 59-Permeability Map for different Training Realizations- Run\#4 
Permeability I (md) 2000-01-01 K layer: 1

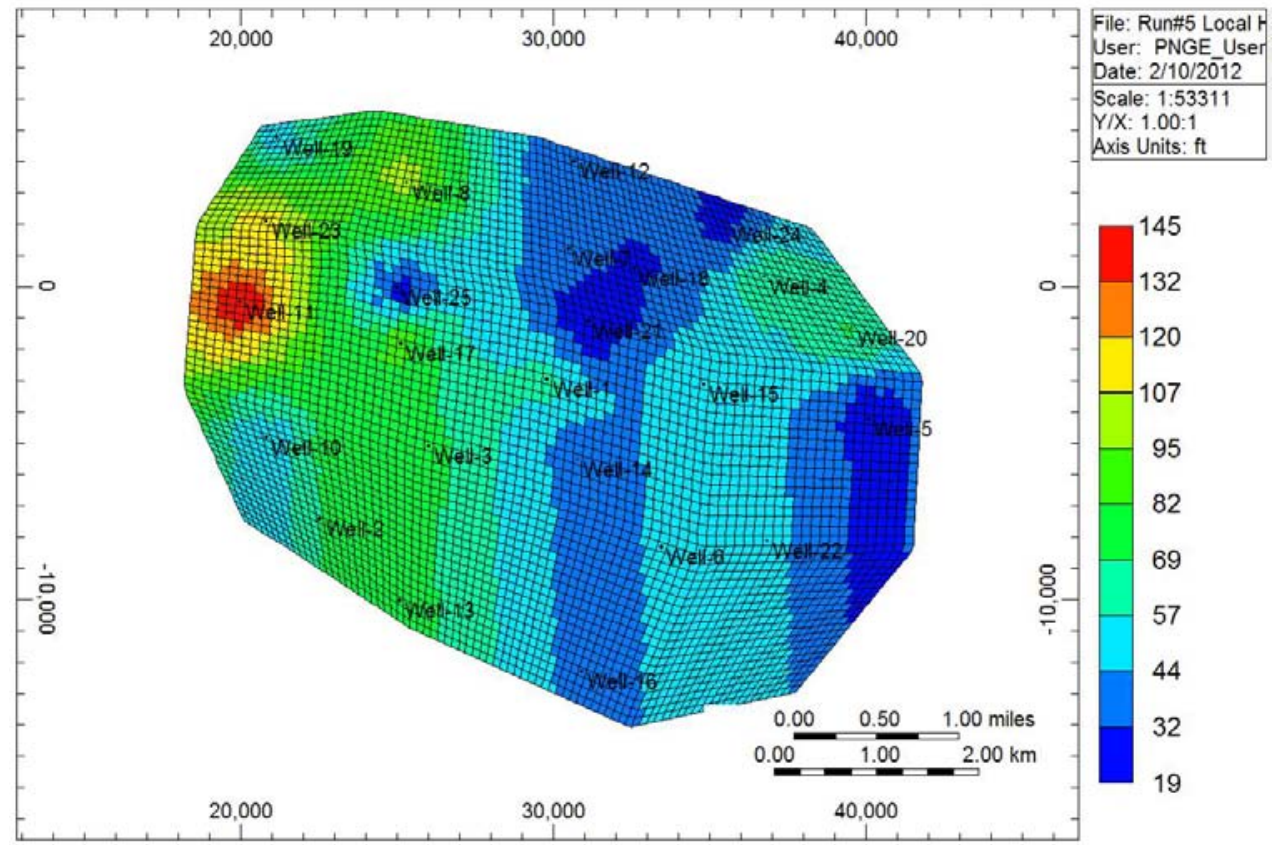

Figure 60-Permeability Map for different Training Realizations- Run\#5

Permeability I (md) 2000-01-01 K layer: 1

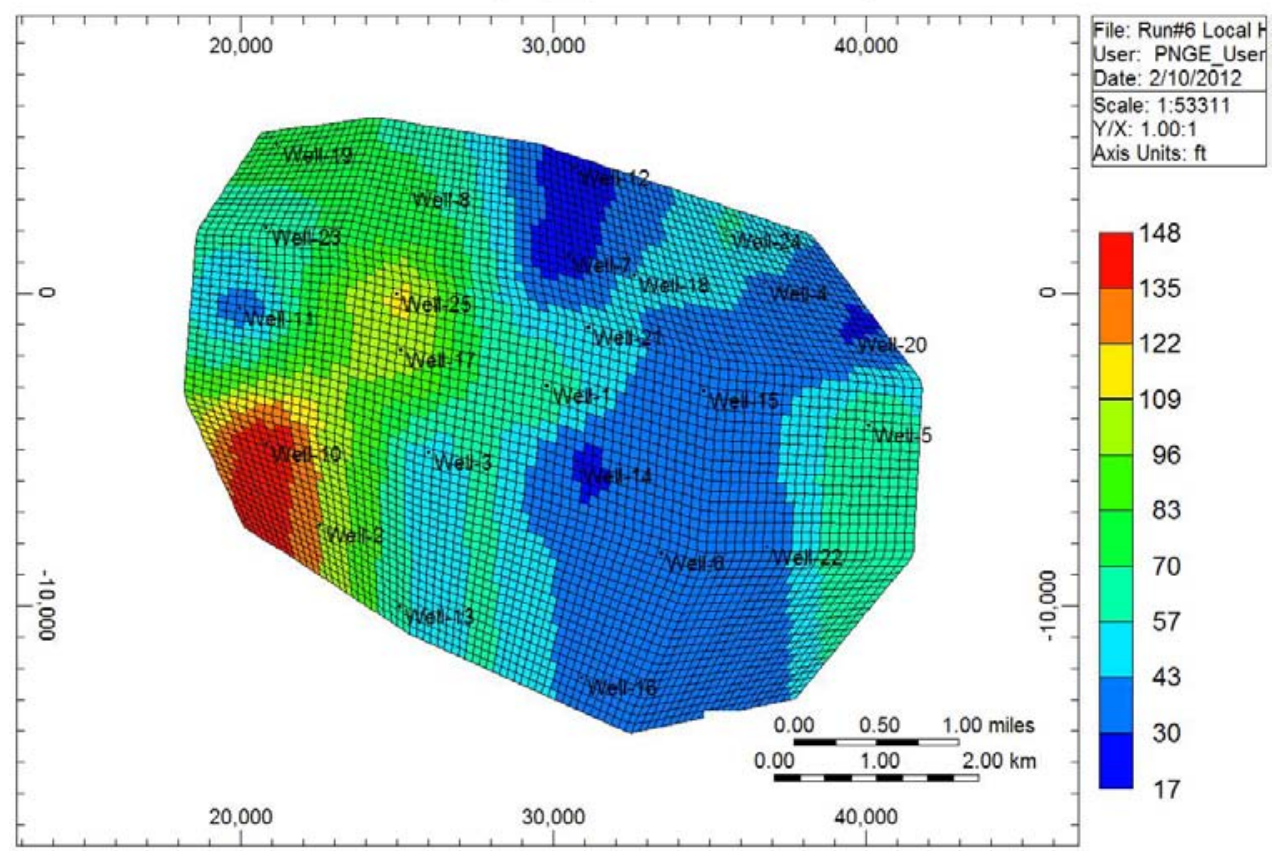

Figure 61-Permeability Map for different Training Realizations- Run\#6 
Permeability I (md) 2000-01-01 K layer: 1

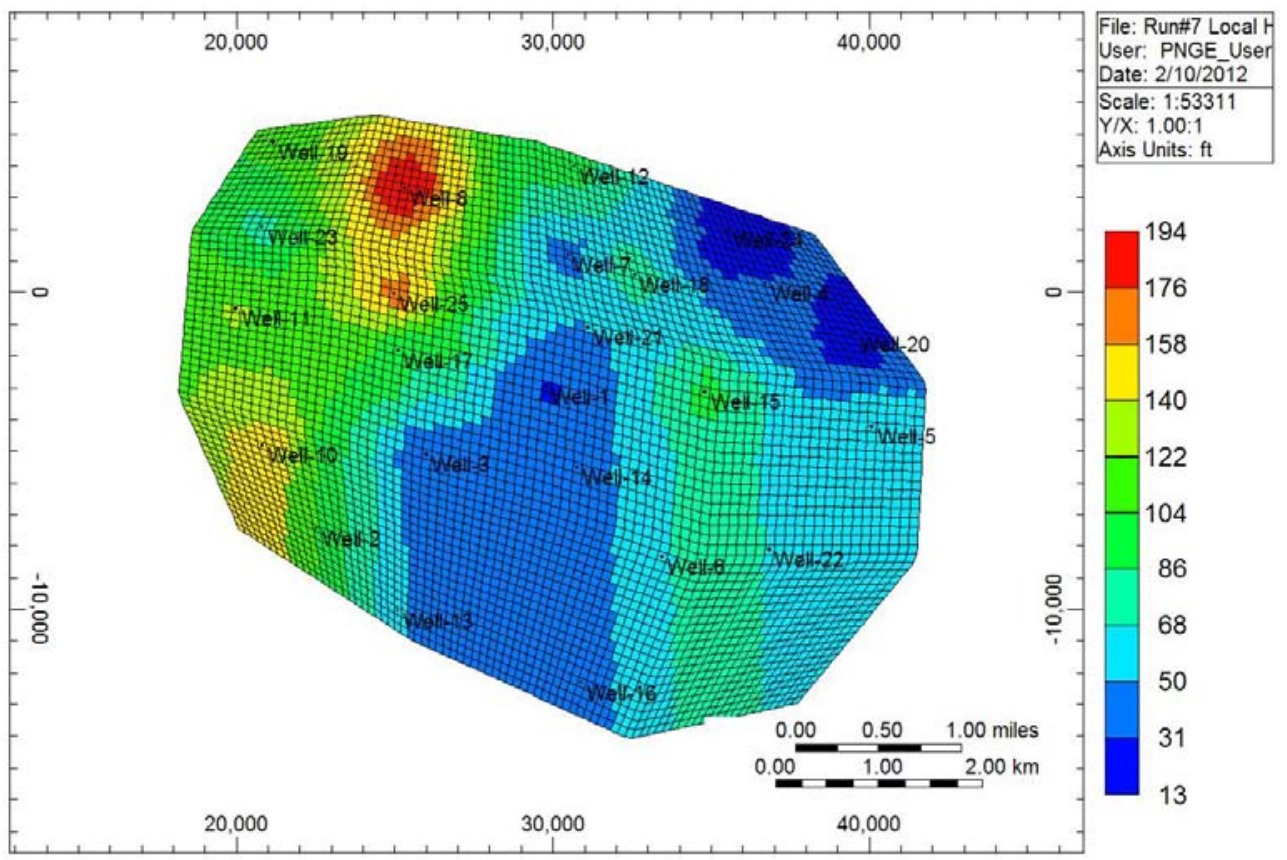

Figure 62-Permeability Map for different Training Realizations- Run\#7

Permeability I (md) 2000-01-01 K layer: 1

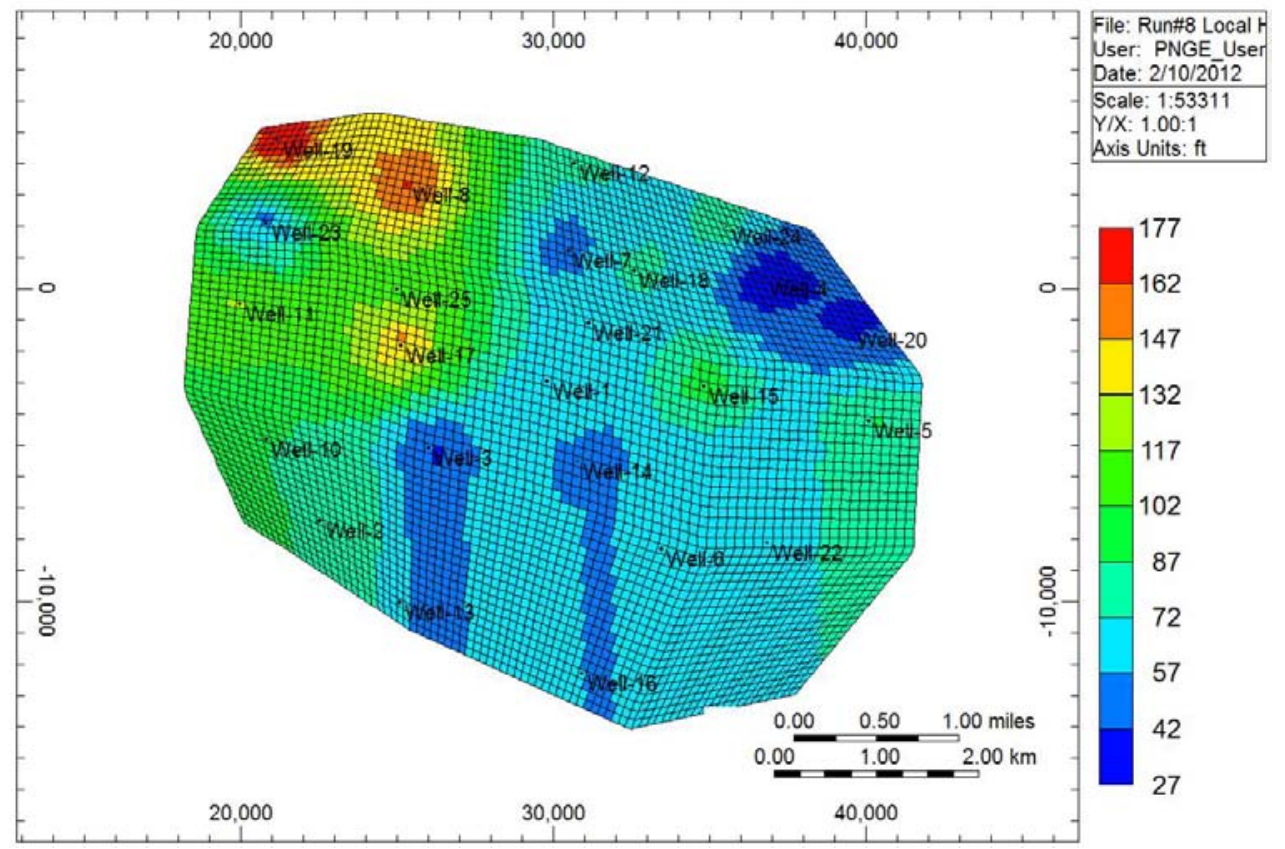

Figure 63-Permeability Map for different Training Realizations- Run\#8 
Permeability I (md) 2000-01-01 K layer: 1

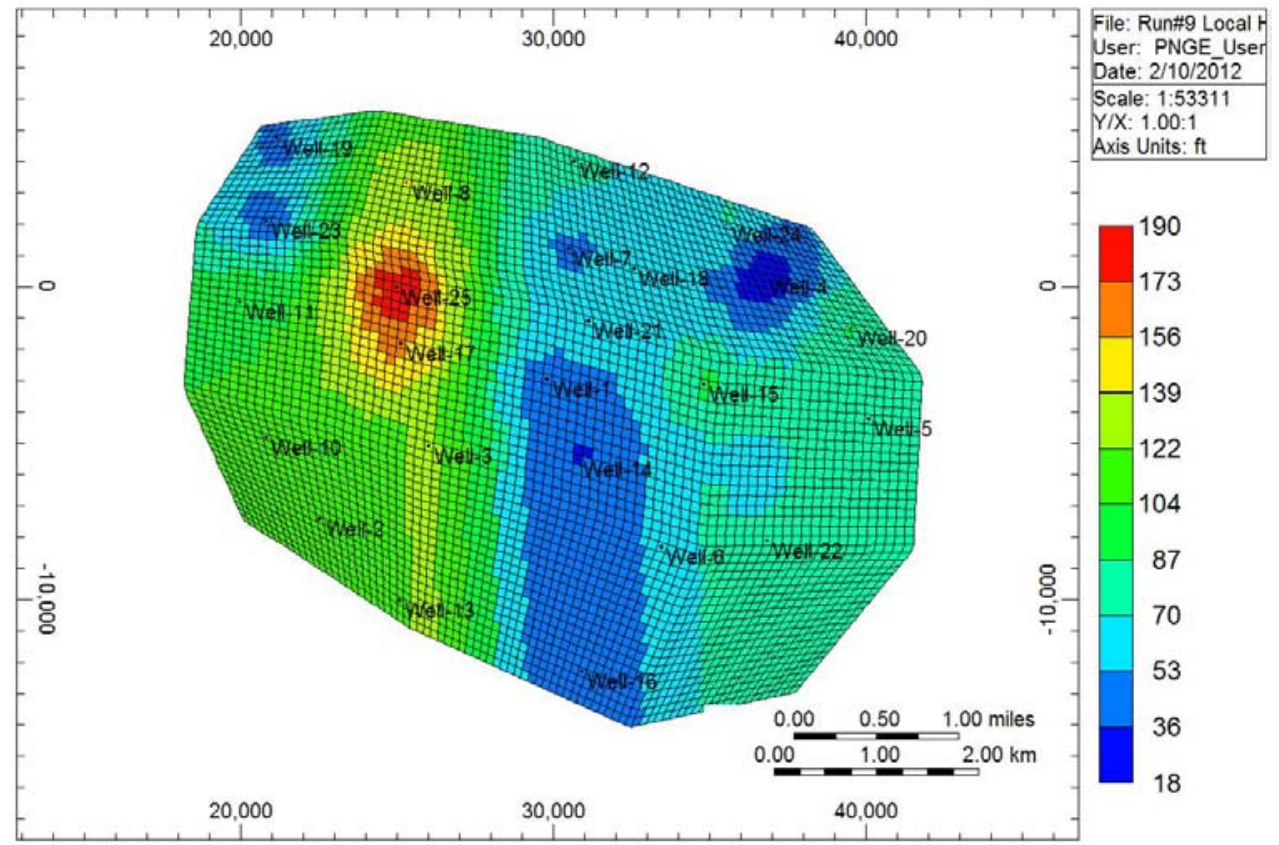

Figure 64-Permeability Map for different Training Realizations- Run\#9

Permeability I (md) 2000-01-01 K layer: 1

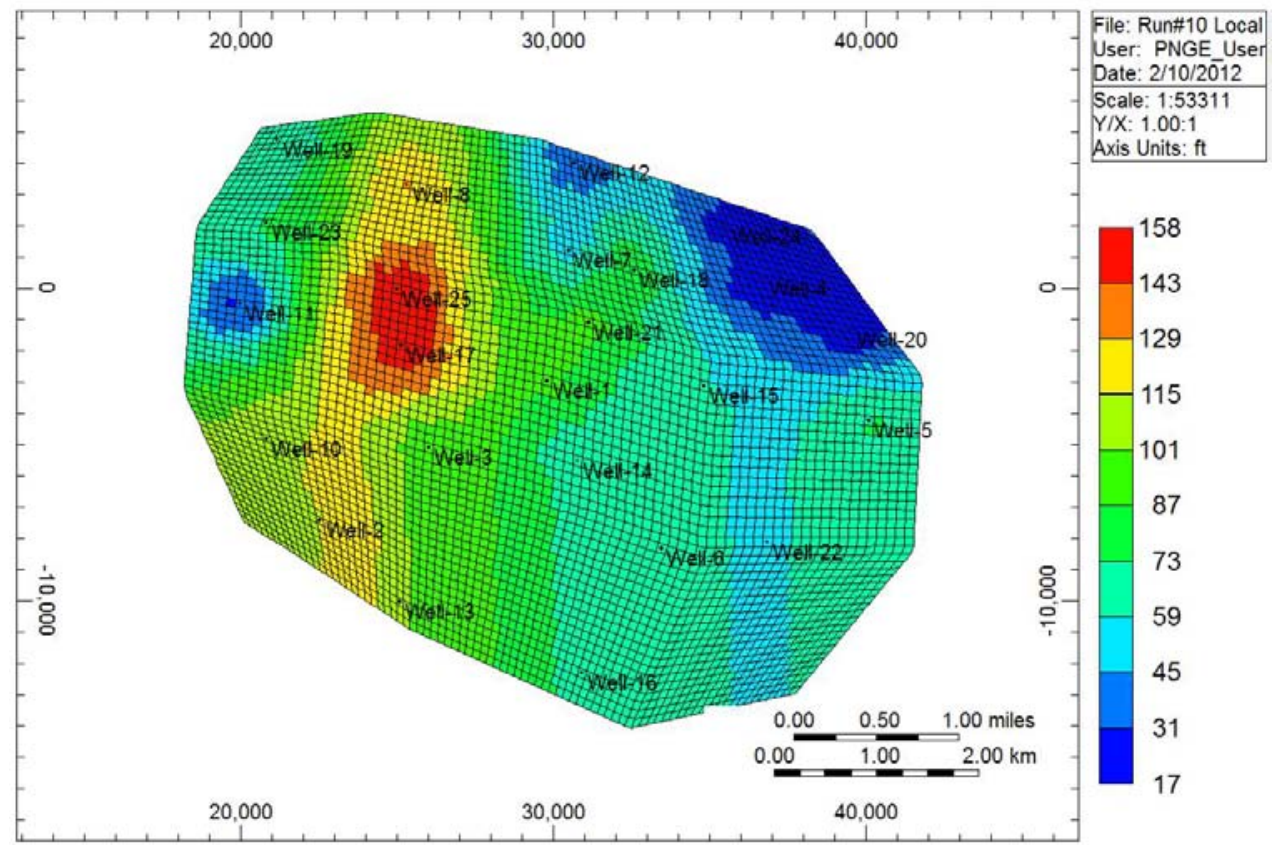

Figure 65-Permeability Map for different Training Realizations- Run\#10 


\section{Appendix C- Results}

\section{a. SRM results for training, calibration and verification using $\mathbf{1 0}$ realizations:}

\section{a.1 - Realization \# 1:}
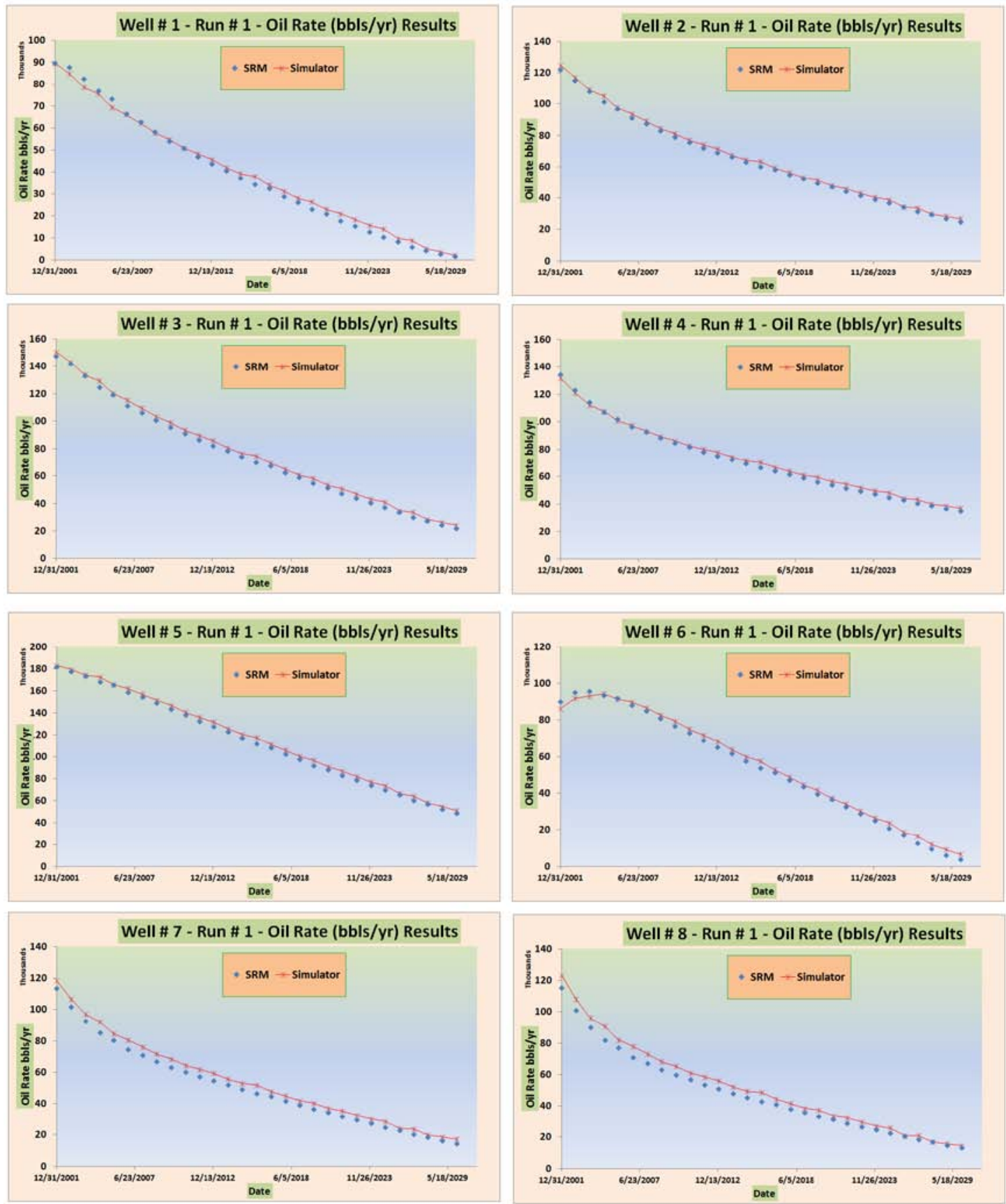

Figure 66- Comparison of the SRM results with simulator outputs after the training process- Run \# 1 

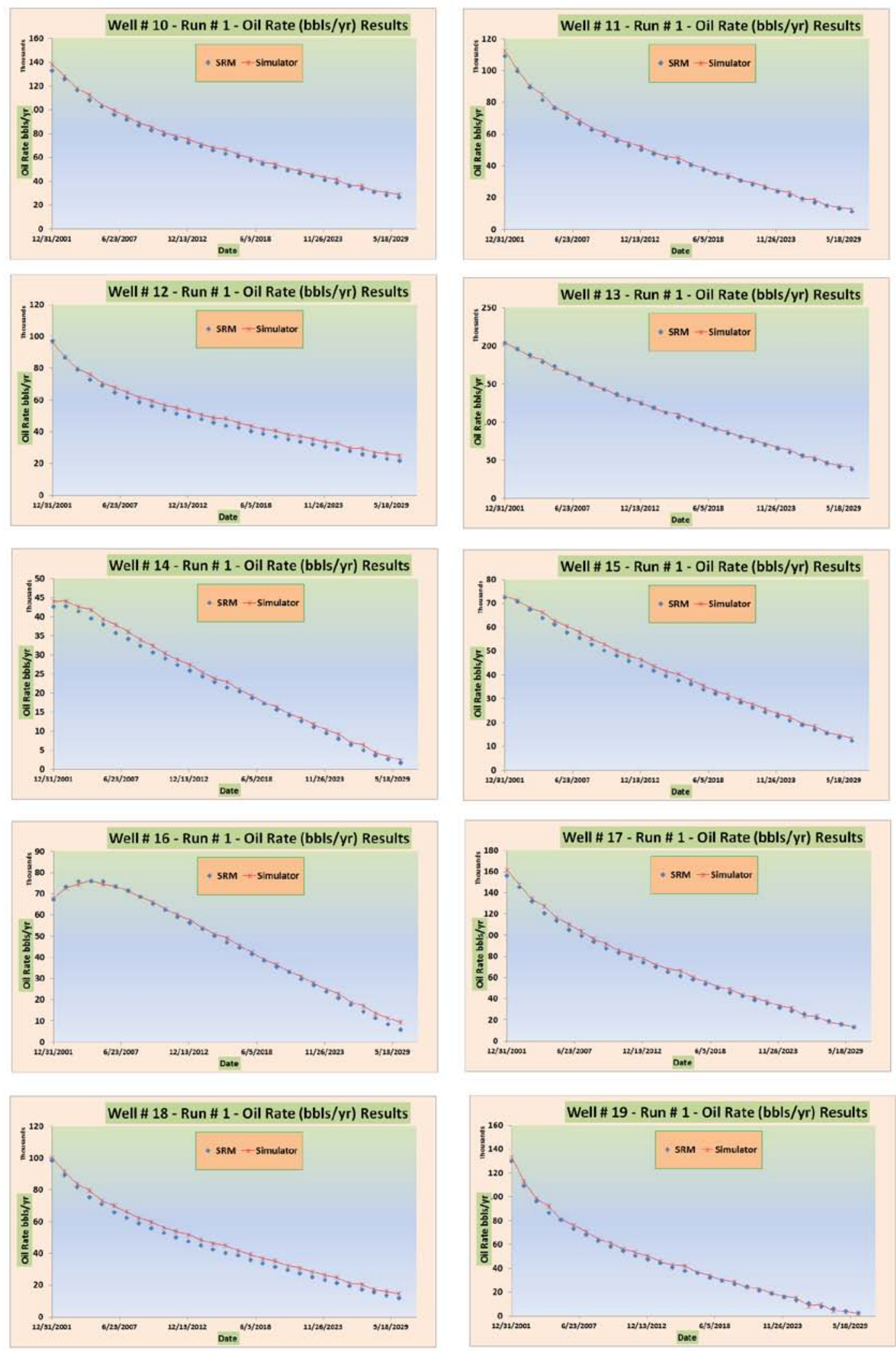

Figure 67- Comparison of the SRM results with simulator outputs after the training process- Run \# 1 

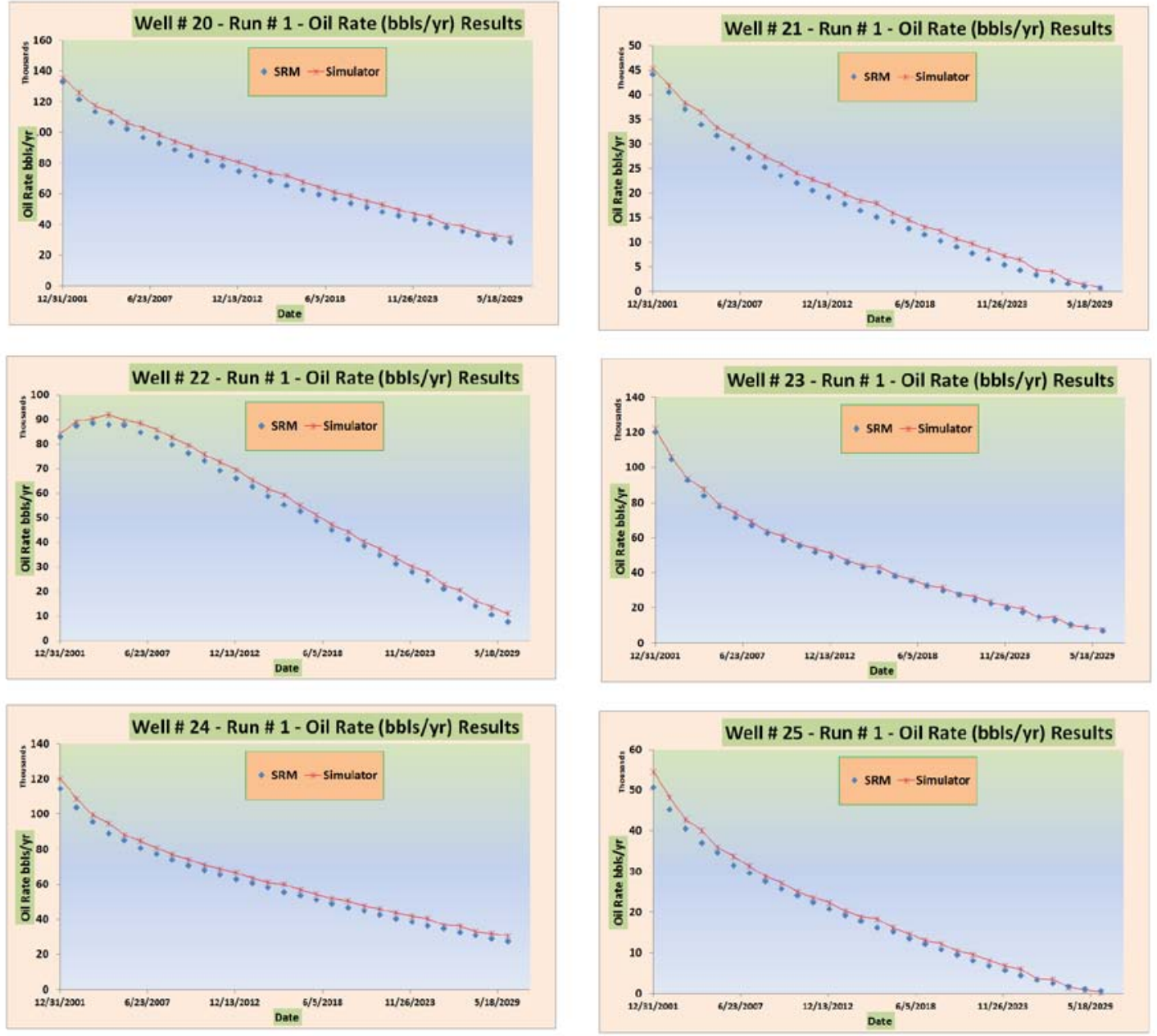

Figure 68- Comparison of the SRM results with simulator outputs after the training process- Run \# 1

\section{a.2 - Realization \# 2:}
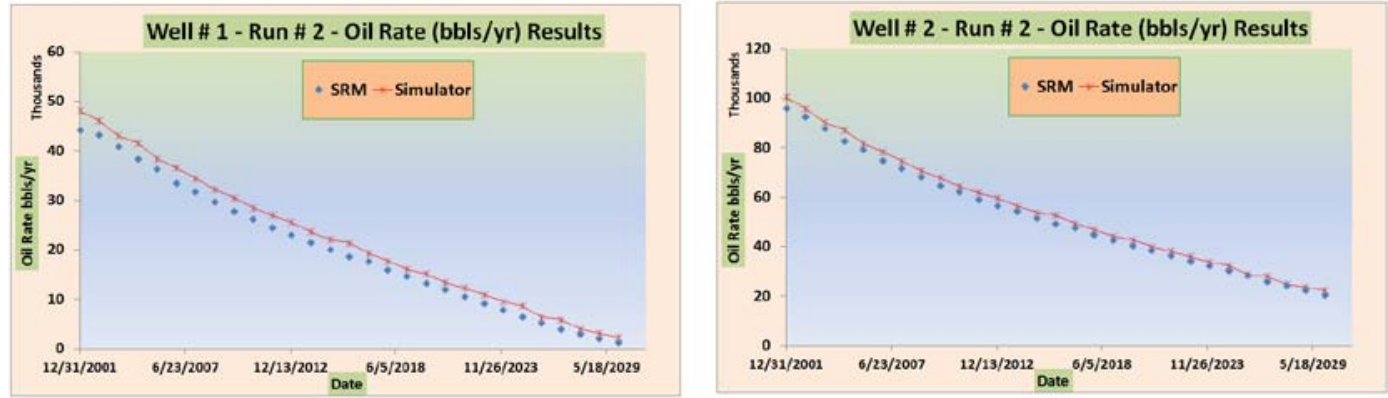

Figure 69- Comparison of the SRM results with simulator outputs after the training process- Run \# 2 

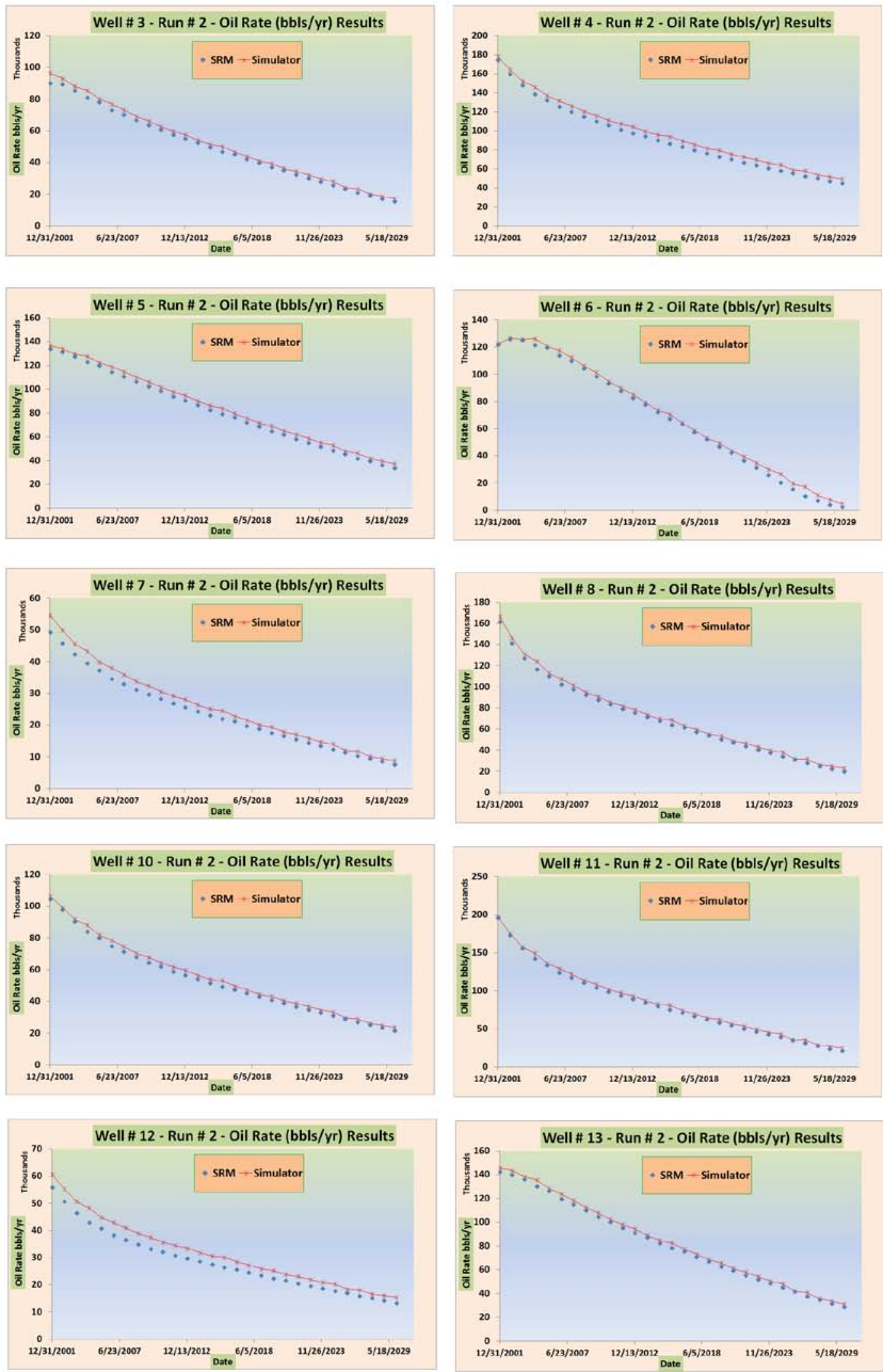

Figure 70- Comparison of the SRM results with simulator outputs after the training process- Run \# 2 

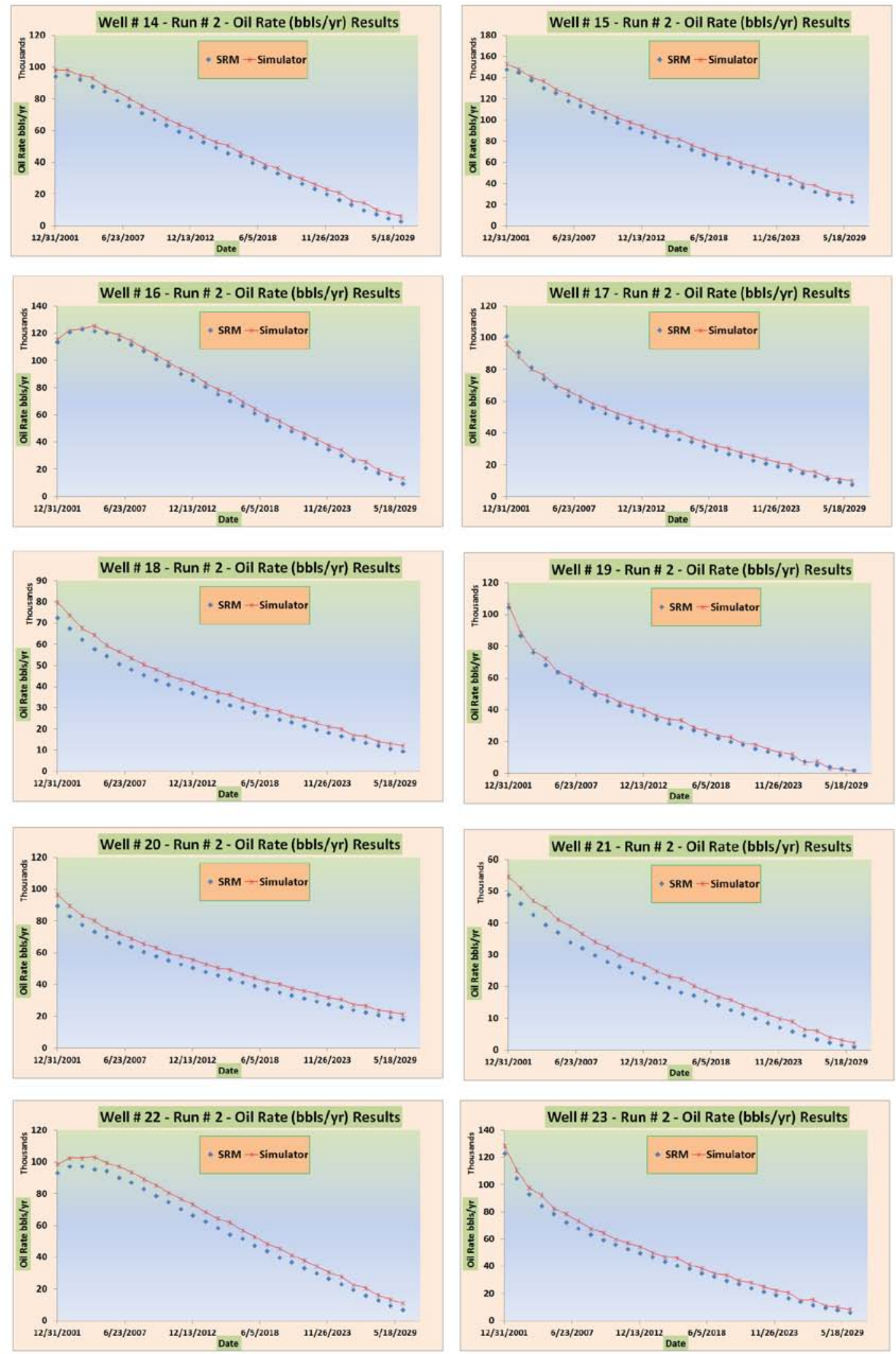

Figure 71- Comparison of the SRM results with simulator outputs after the training process- Run \# 2 

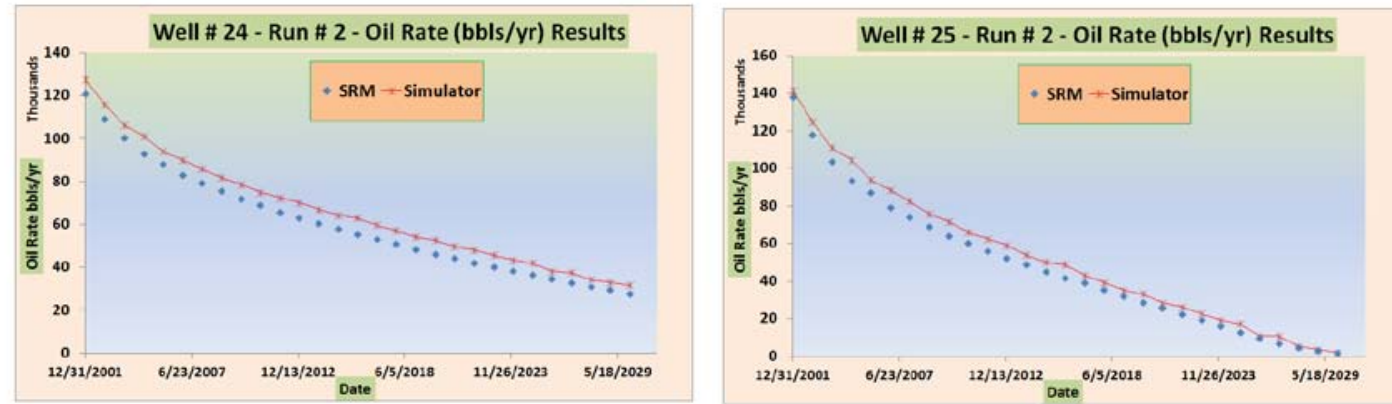

Figure 72- Comparison of the SRM results with simulator outputs after the training process- Run \# 2

\section{a.3 - Realization \# 3:}
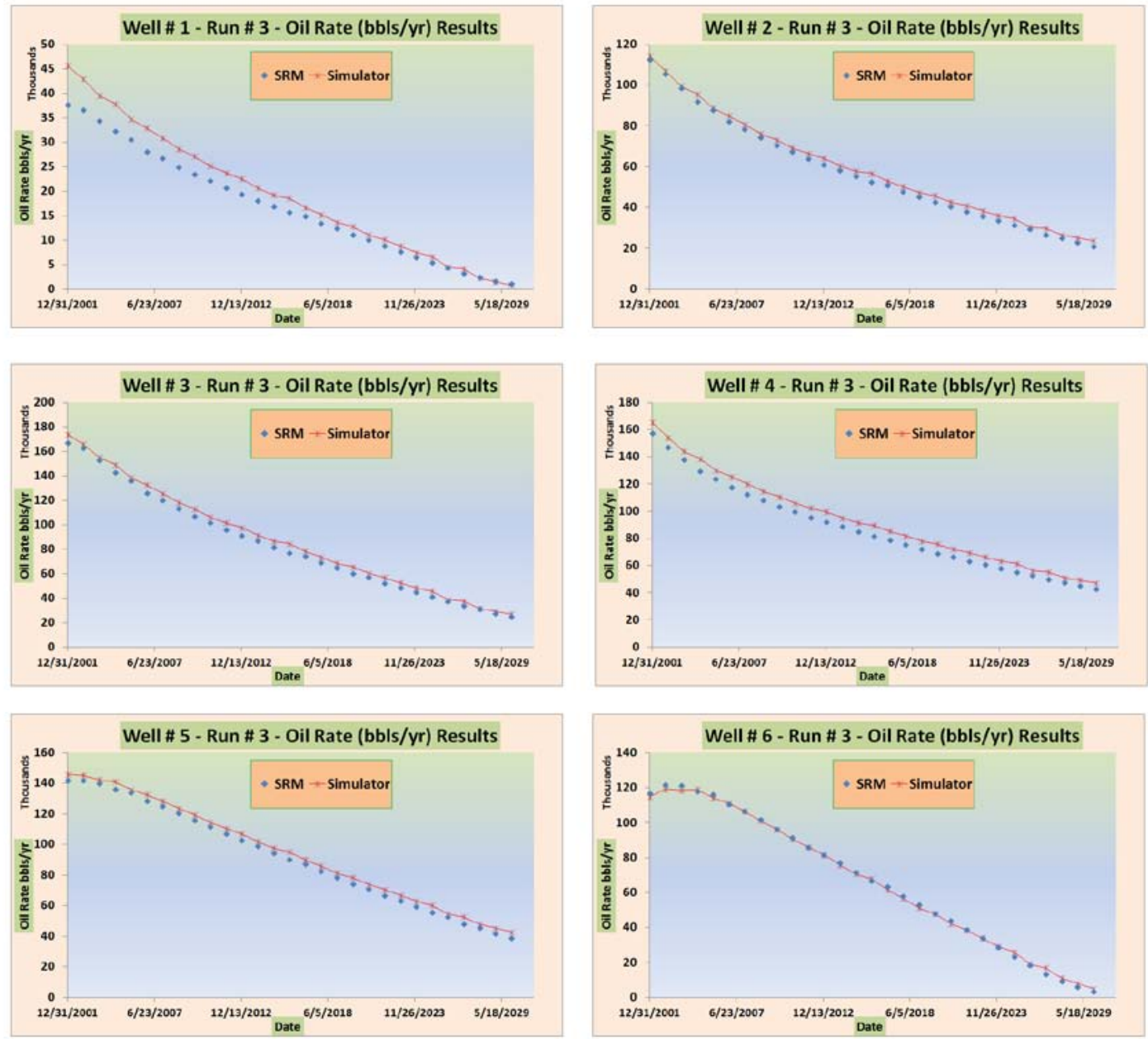

Figure 73- Comparison of the SRM results with simulator outputs after the training process- Run \# 3 

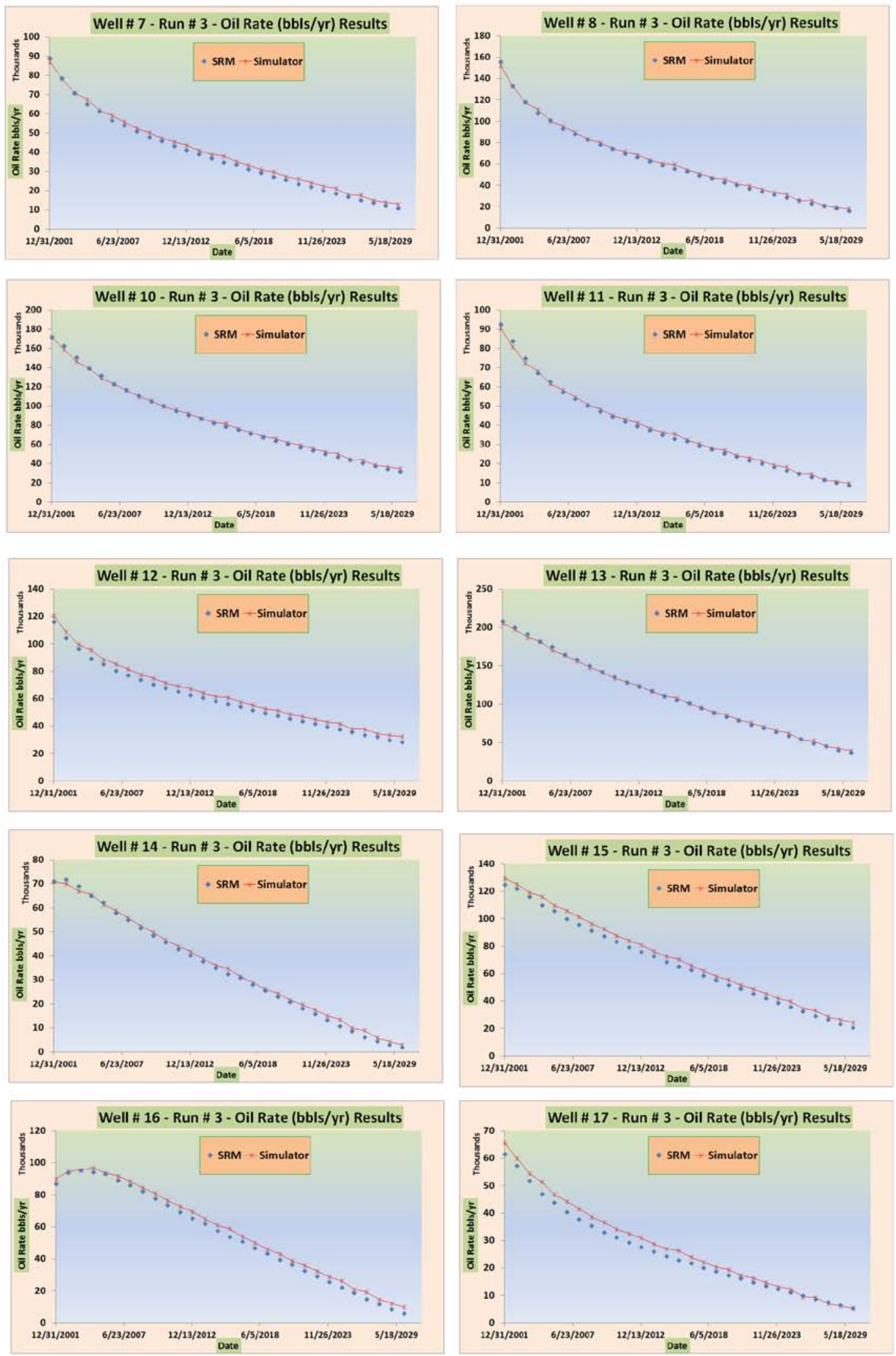

Figure 74- Comparison of the SRM results with simulator outputs after the training process- Run \# 3 

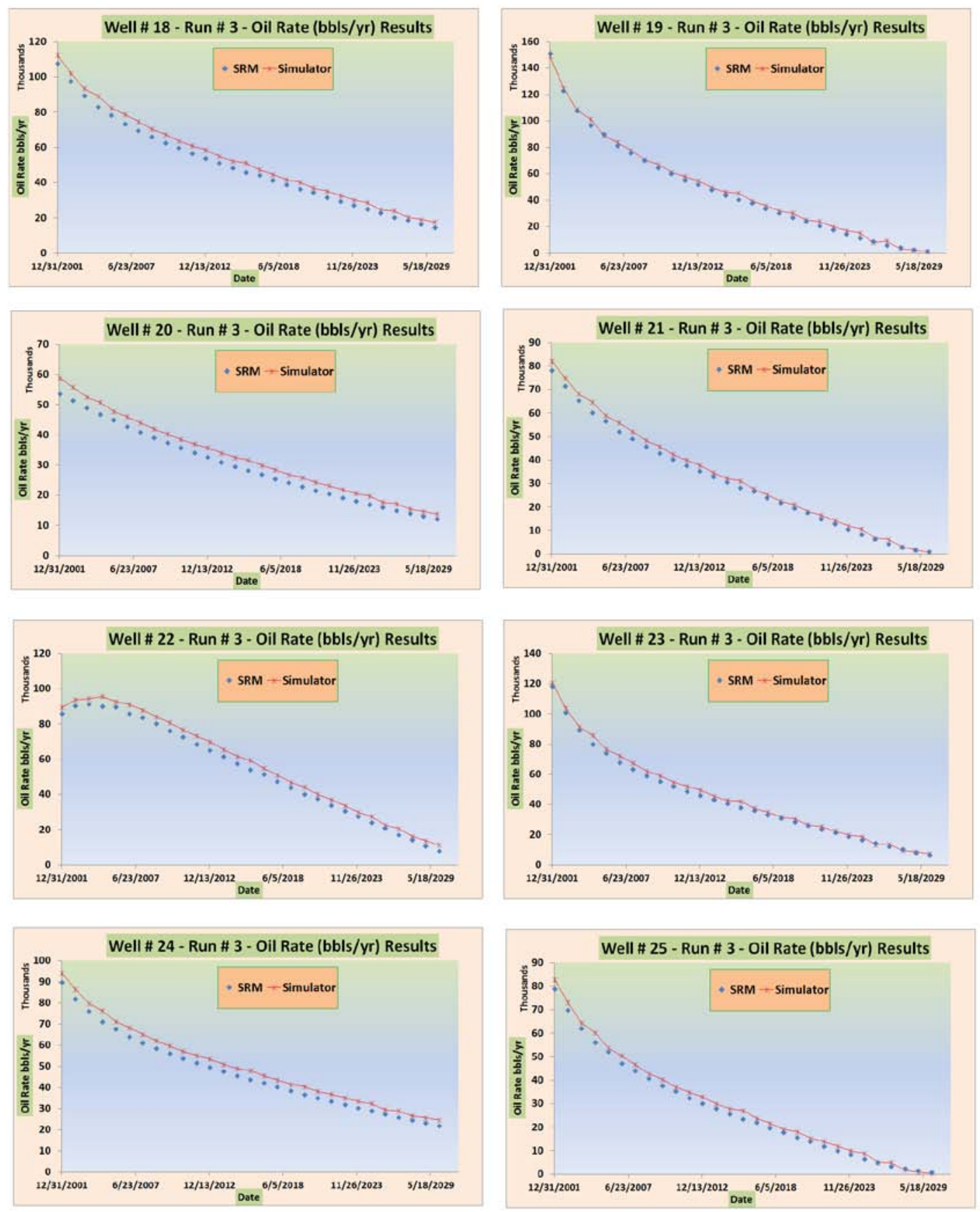

Figure 75- Comparison of the SRM results with simulator outputs after the training process- Run \# 3 


\section{a.4 - Realization \# 4:}
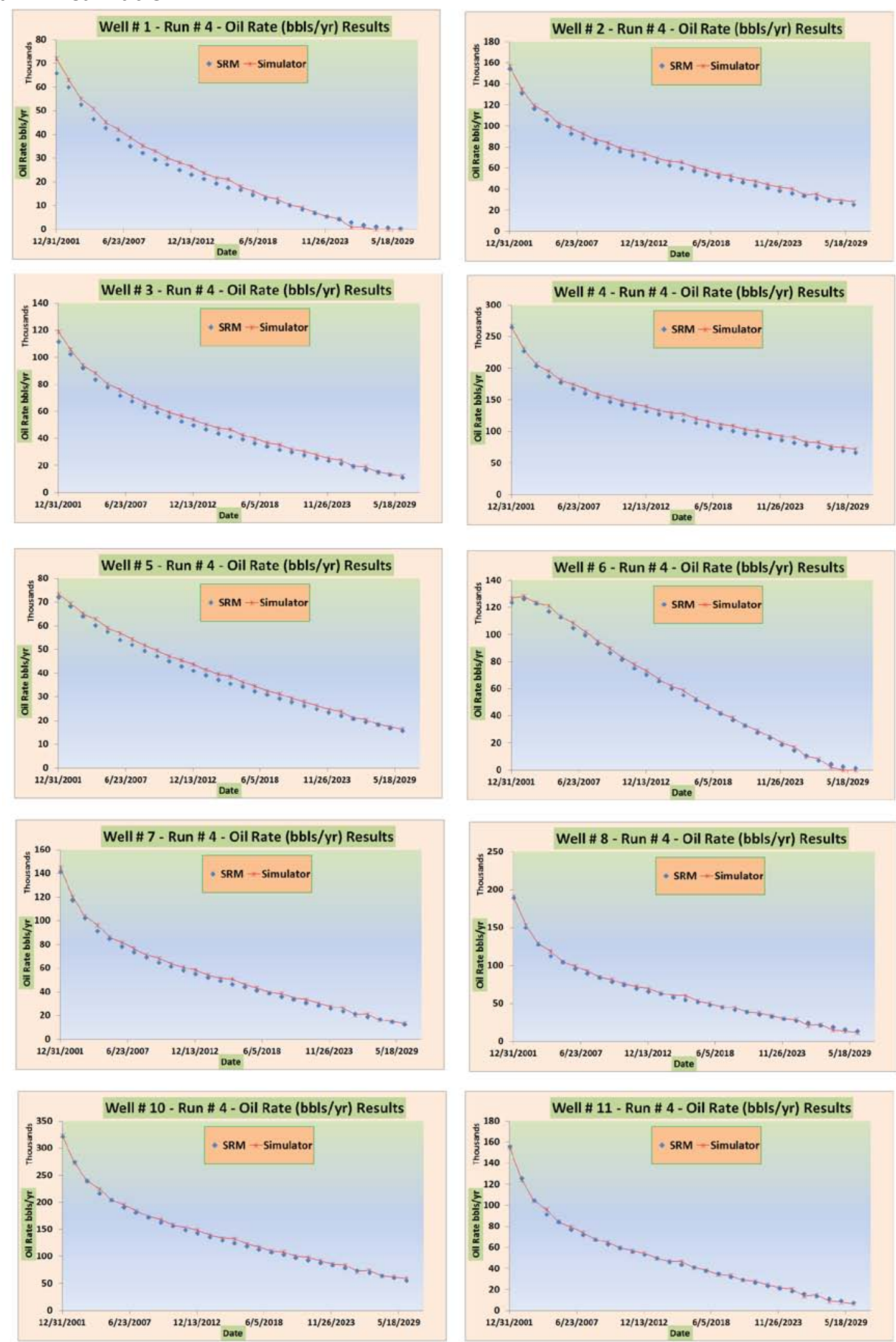

Figure 76- Comparison of the SRM results with simulator outputs after the training process- Run \# 4 

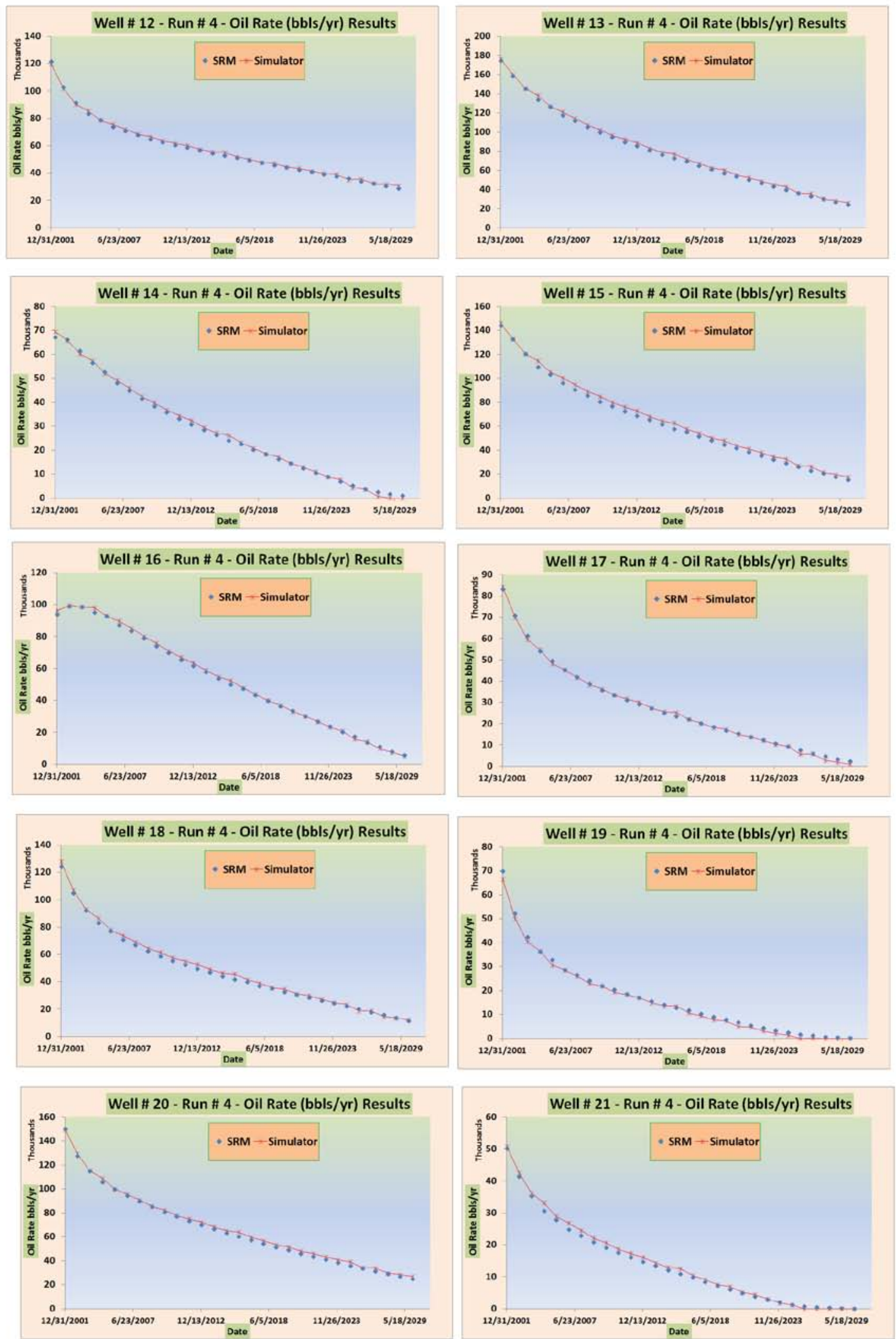

Figure 77- Comparison of the SRM results with simulator outputs after the training process- Run \# 4 

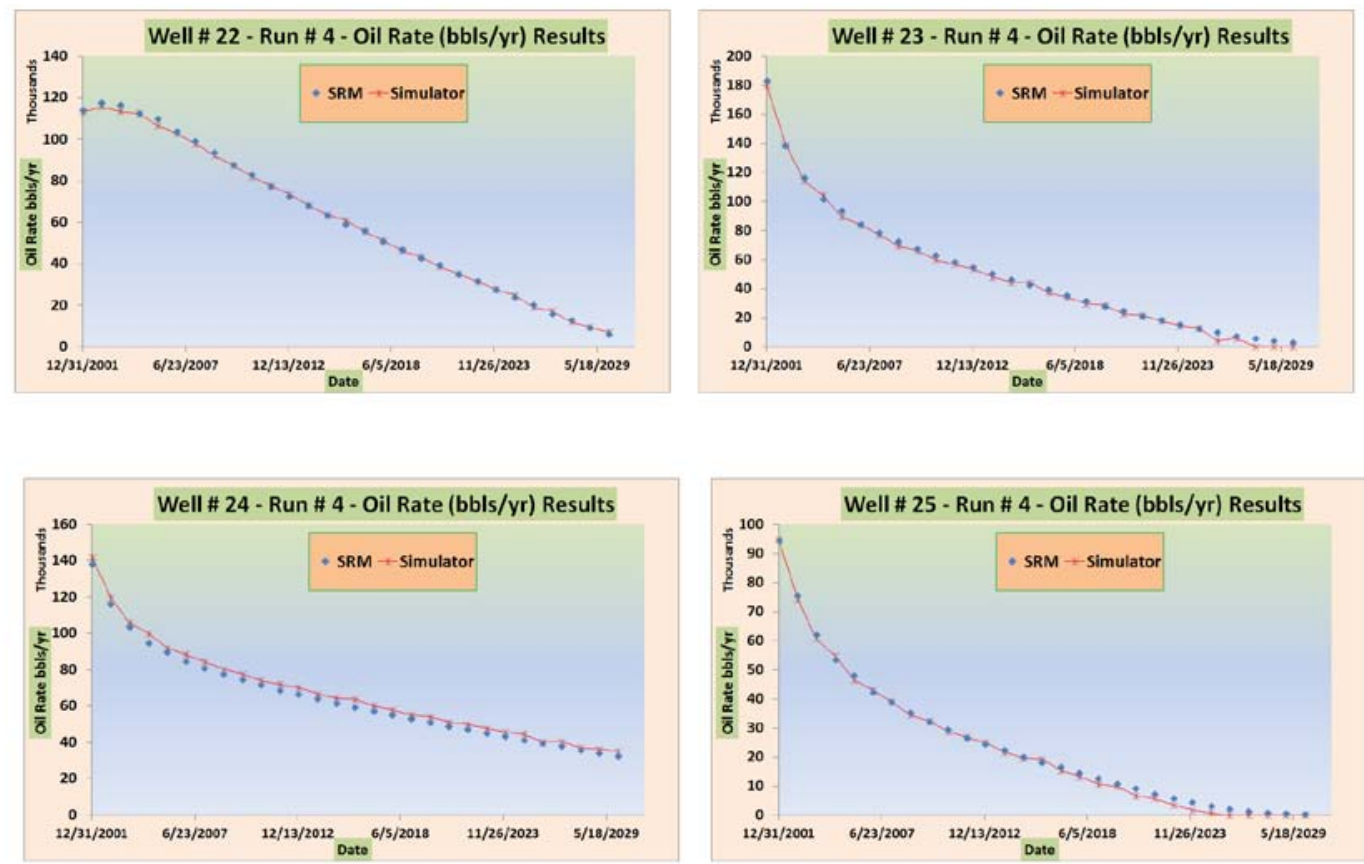

Figure 78- Comparison of the SRM results with simulator outputs after the training process- Run \# 4

\section{a.5 - Realization \# 5:}
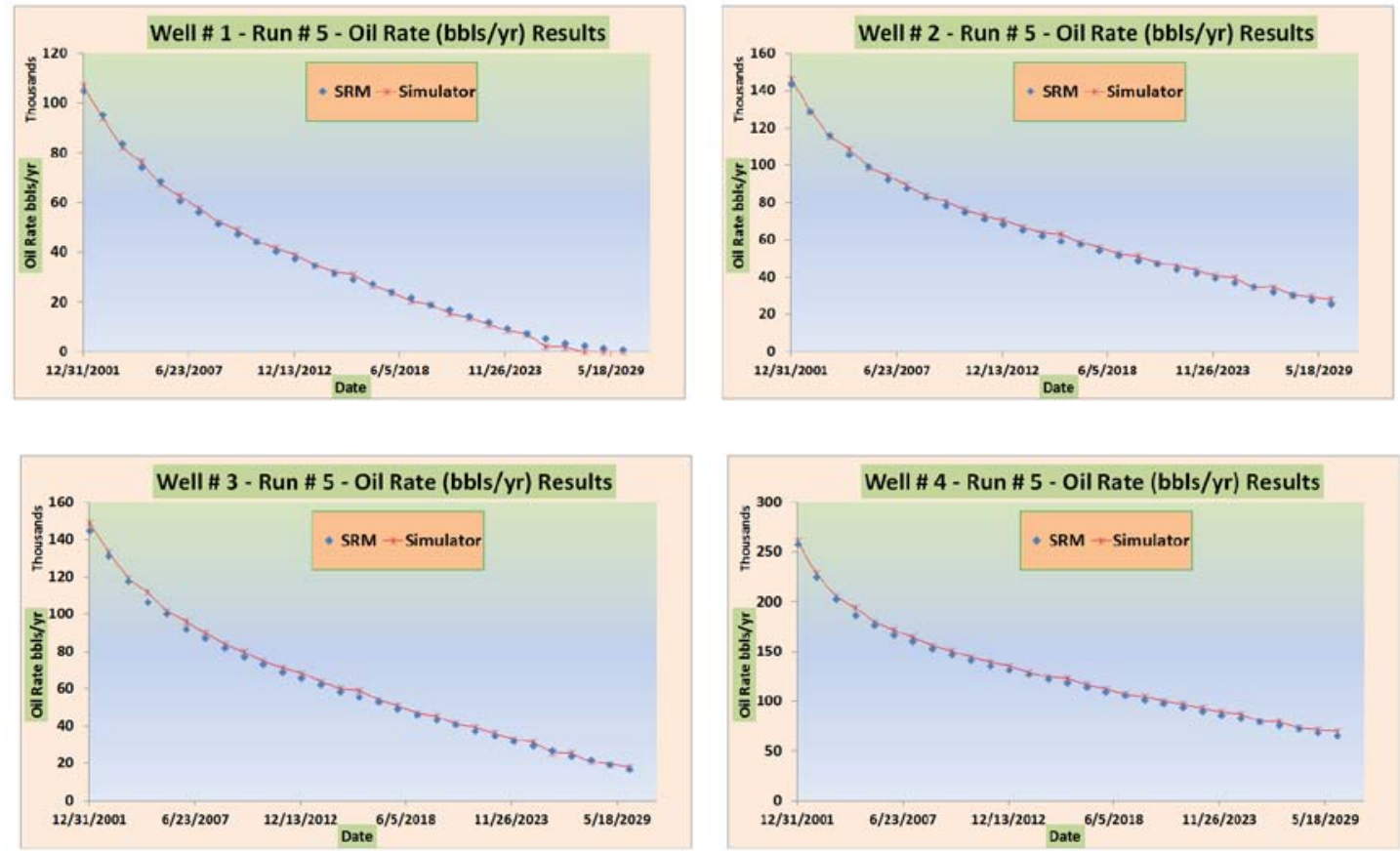

Figure 79- Comparison of the SRM results with simulator outputs after the training process- Run \# 5 

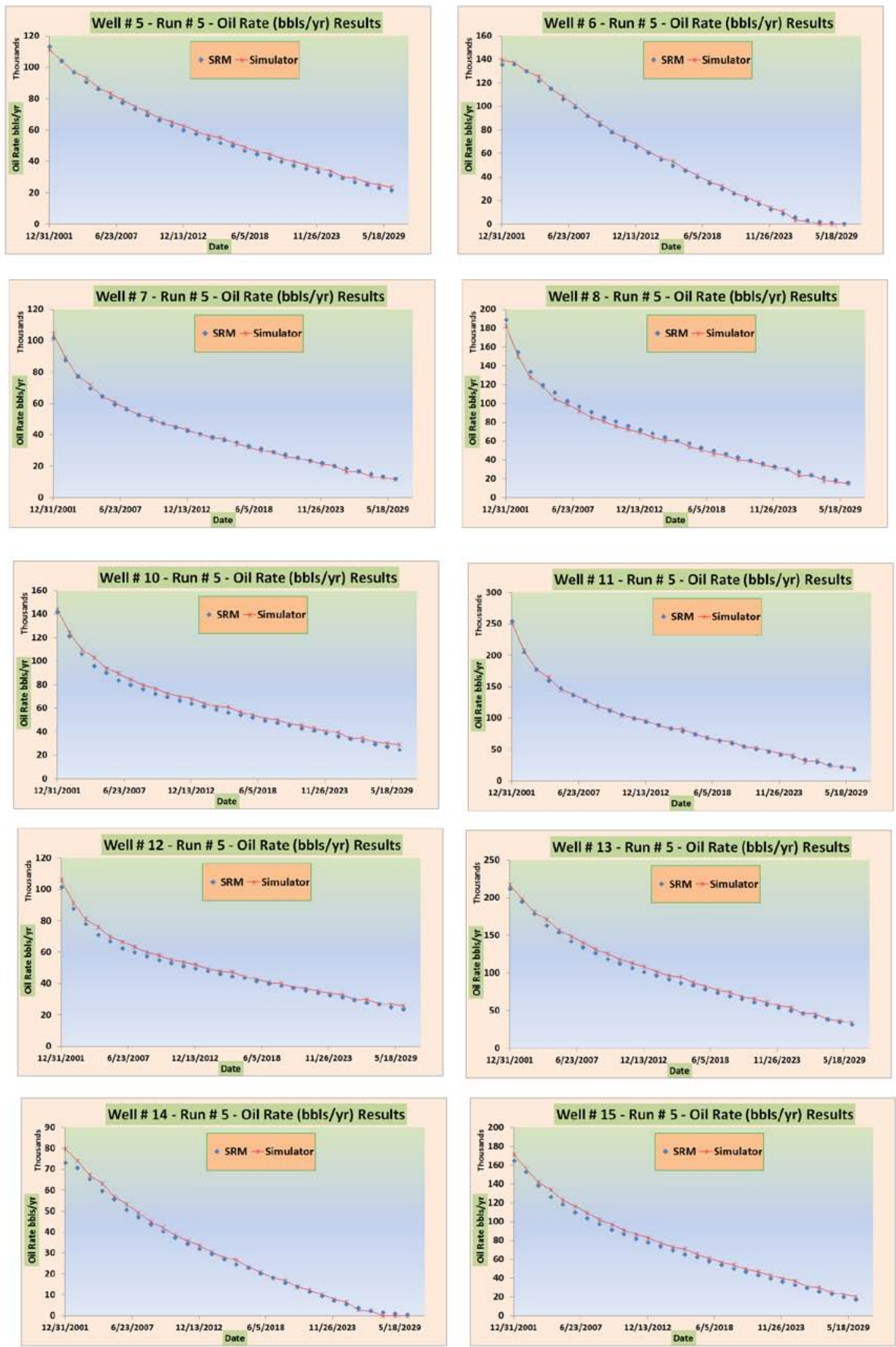

Figure 80- Comparison of the SRM results with simulator outputs after the training process- Run \# 5 

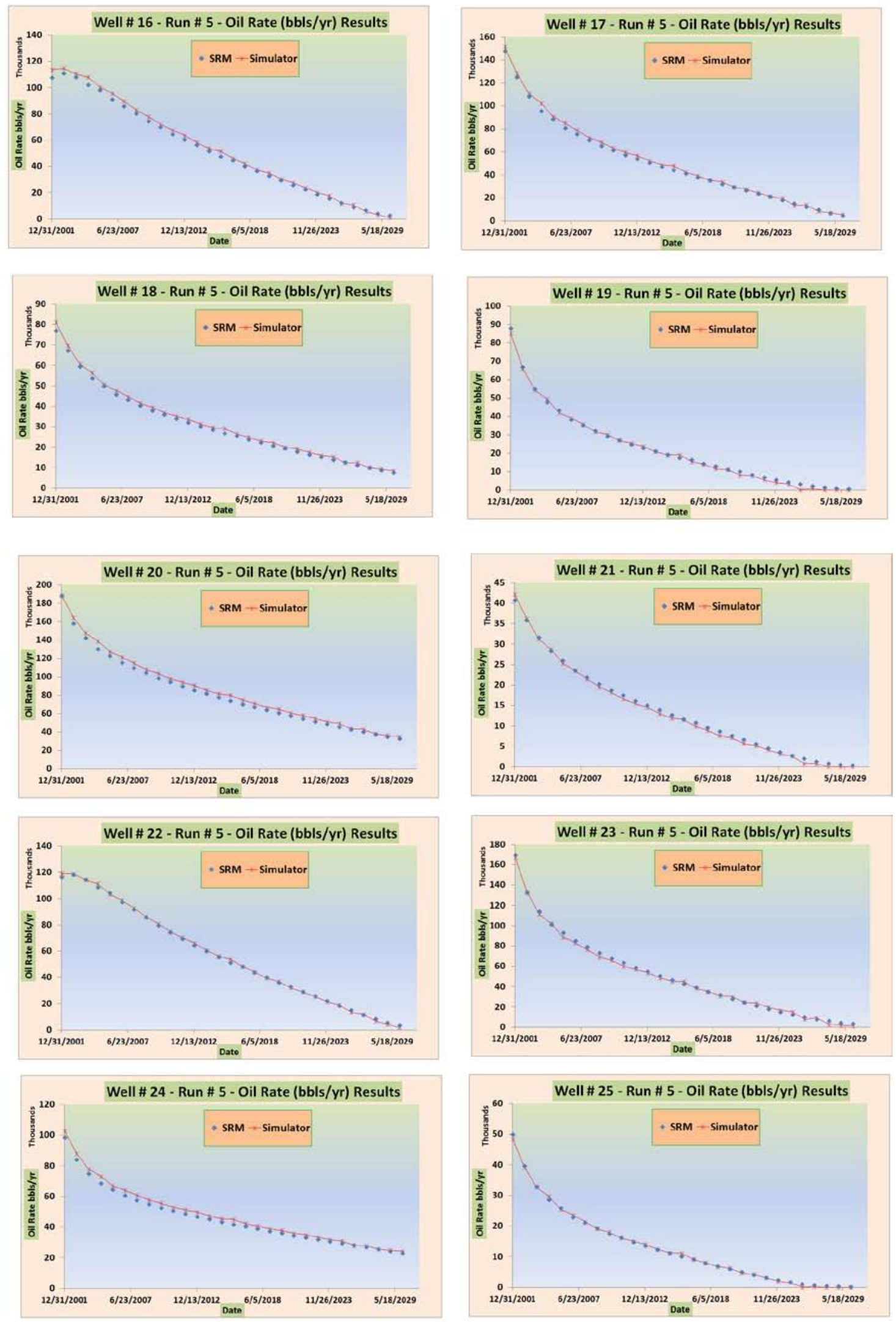

Figure 81- Comparison of the SRM results with simulator outputs after the training process- Run \# 5 


\section{a.6 - Realization \# 6:}
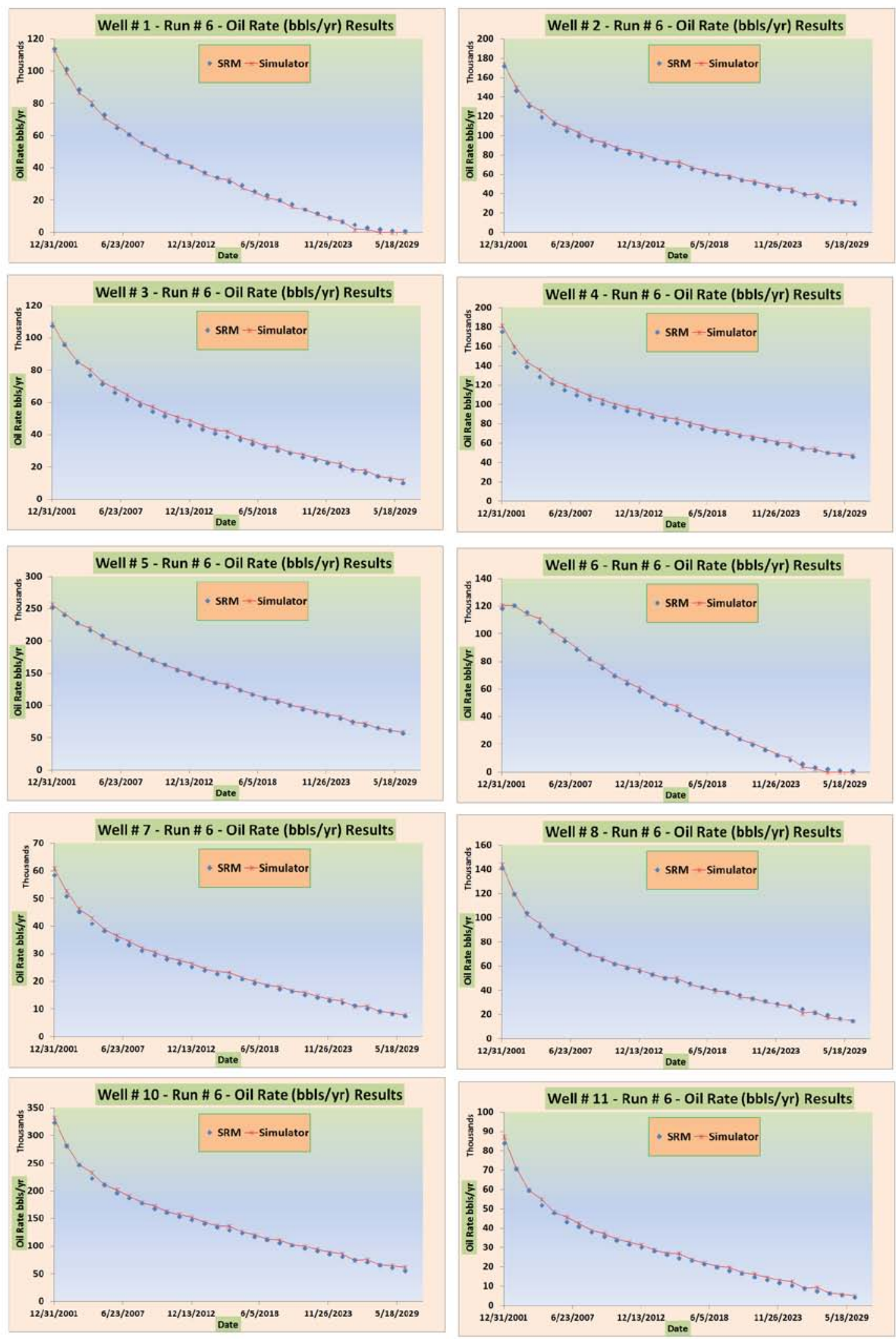

Figure 82- Comparison of the SRM results with simulator outputs after the training process- Run \# 6 

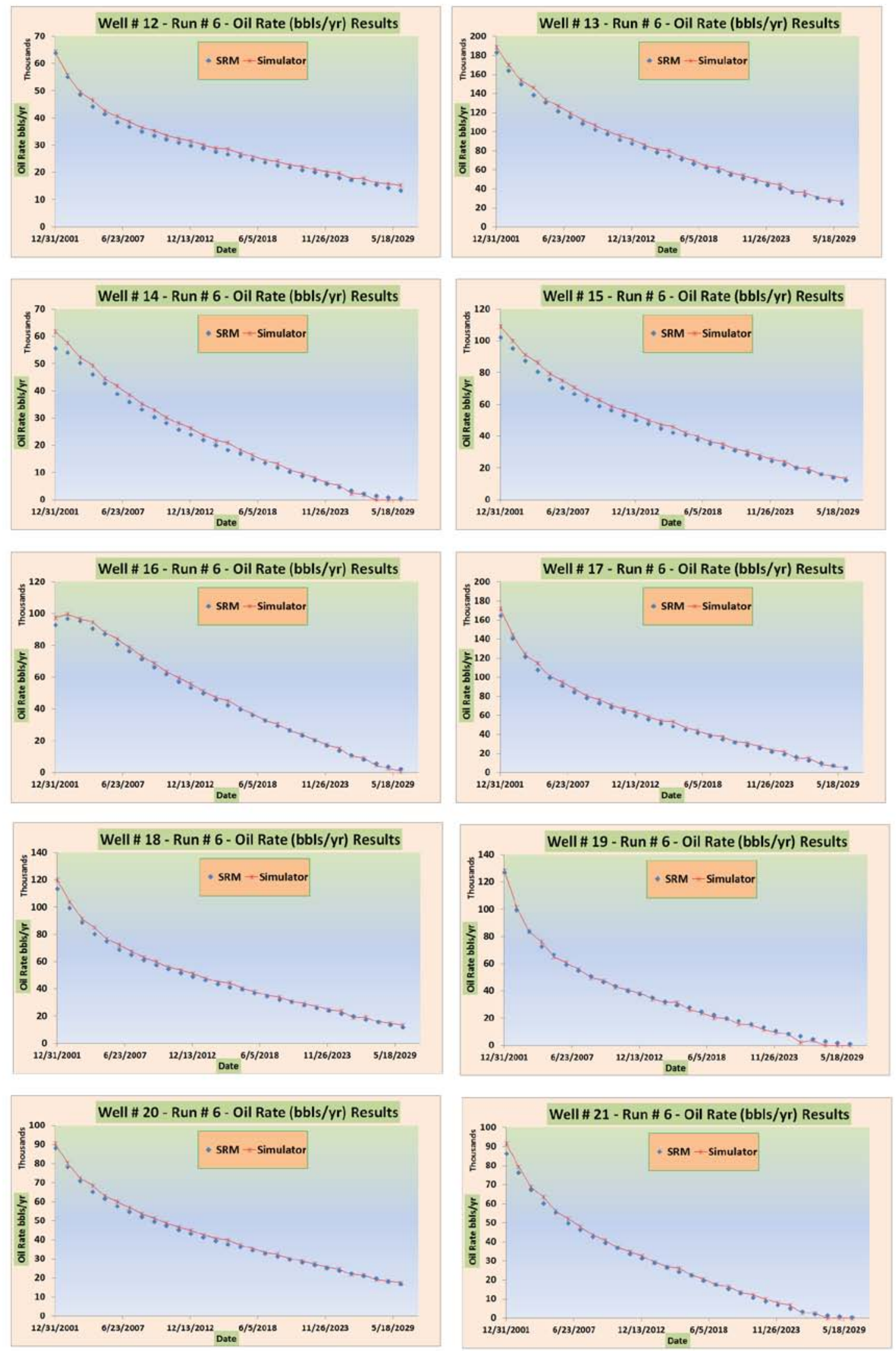

Figure 83- Comparison of the SRM results with simulator outputs after the training process- Run \# 6 

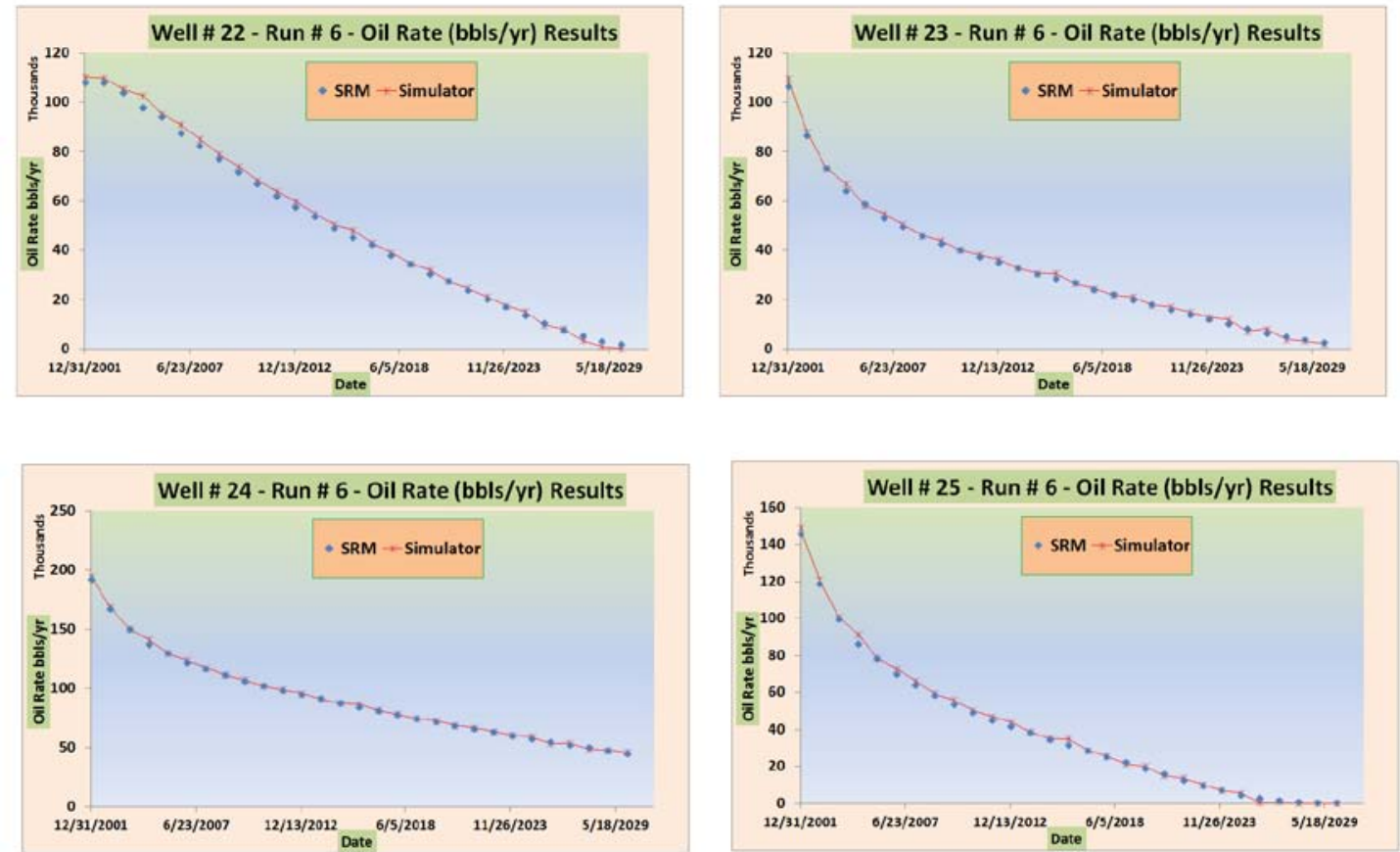

Figure 84- Comparison of the SRM results with simulator outputs after the training process- Run \# 6

\section{a.7 - Realization \# 7:}
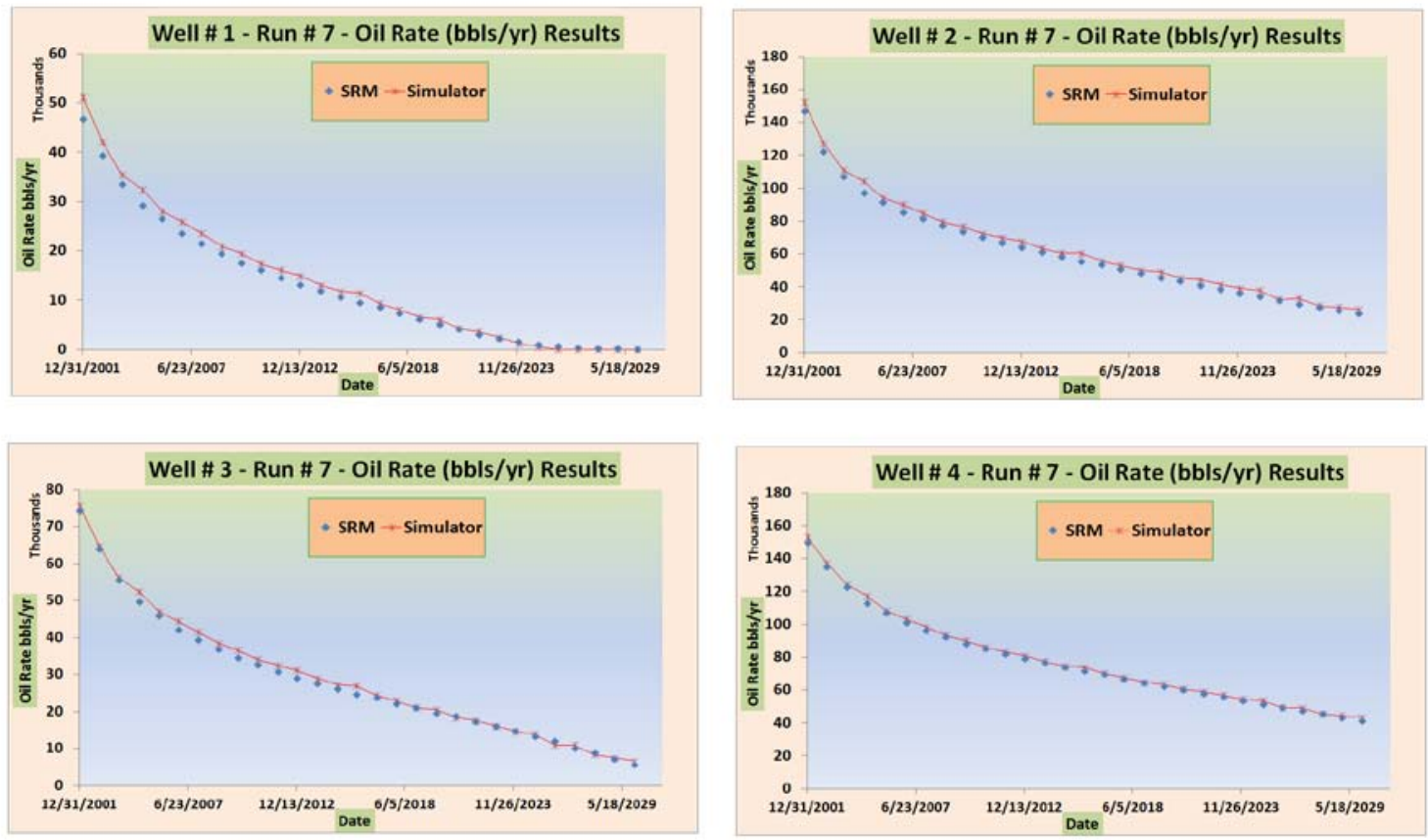

Figure 85- Comparison of the SRM results with simulator outputs after the training process- Run \# 7 

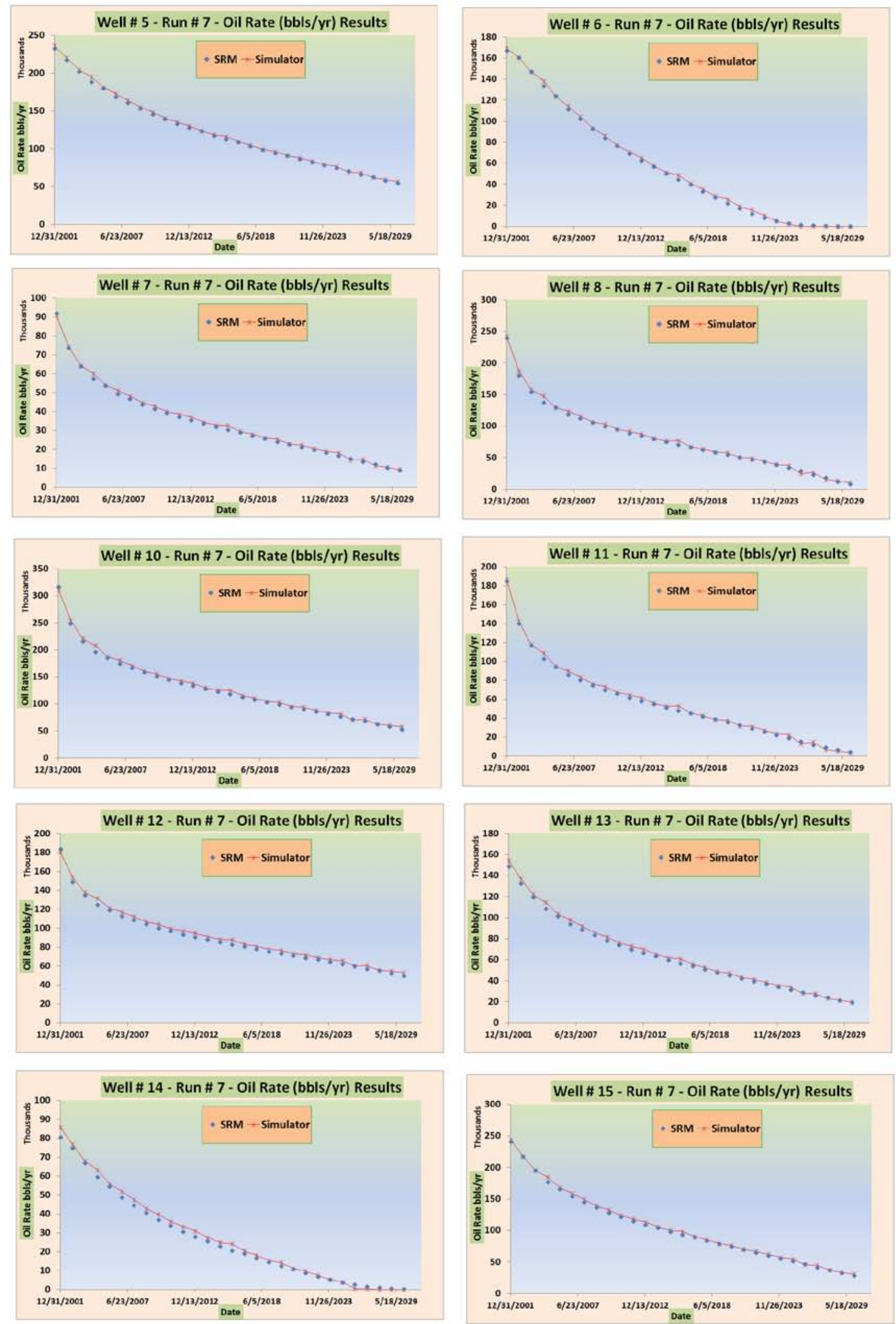

Figure 86- Comparison of the SRM results with simulator outputs after the training process- Run \# 7 

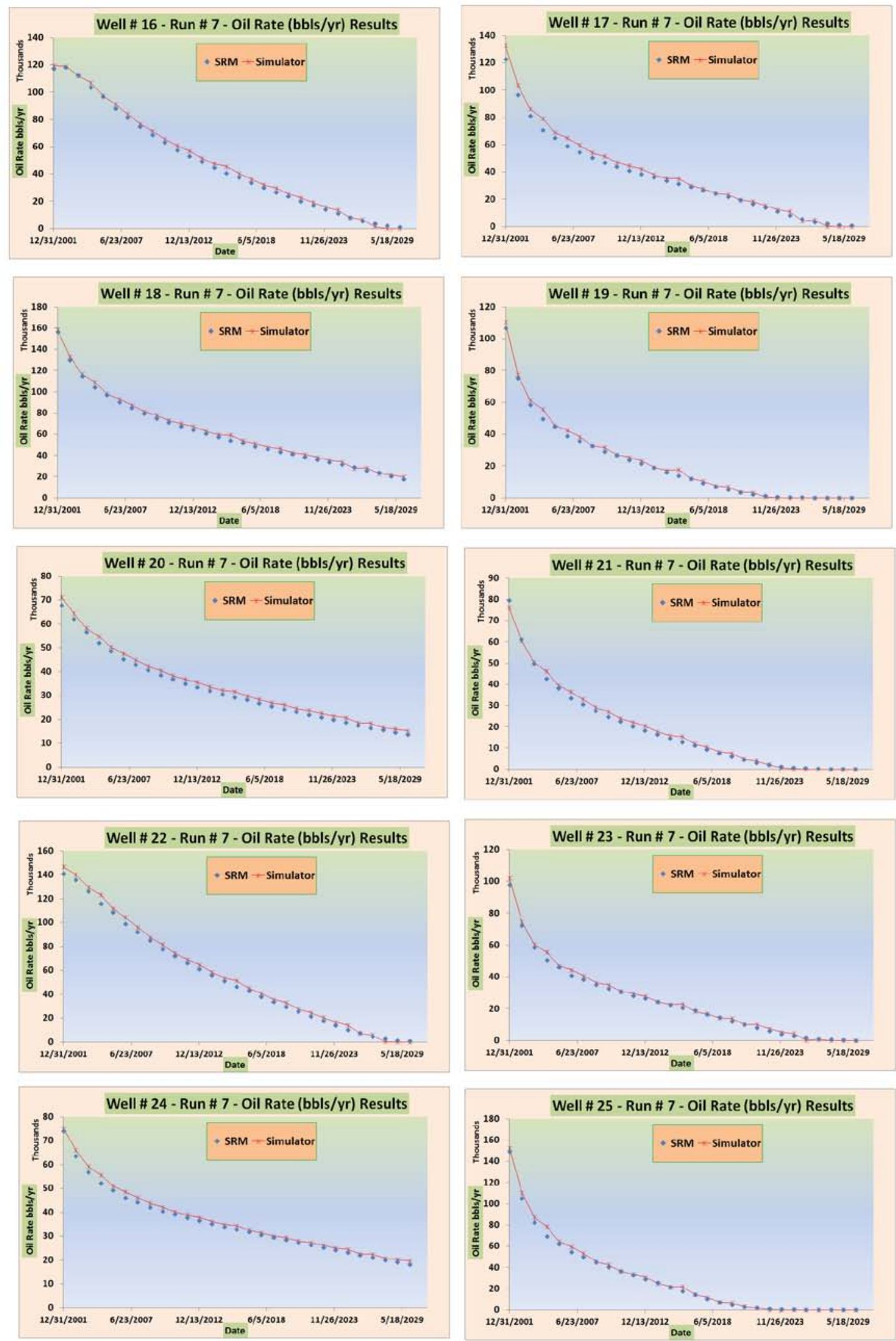

Figure 87- Comparison of the SRM results with simulator outputs after the training process- Run \# 7 


\section{a.8 - Realization \# 8:}
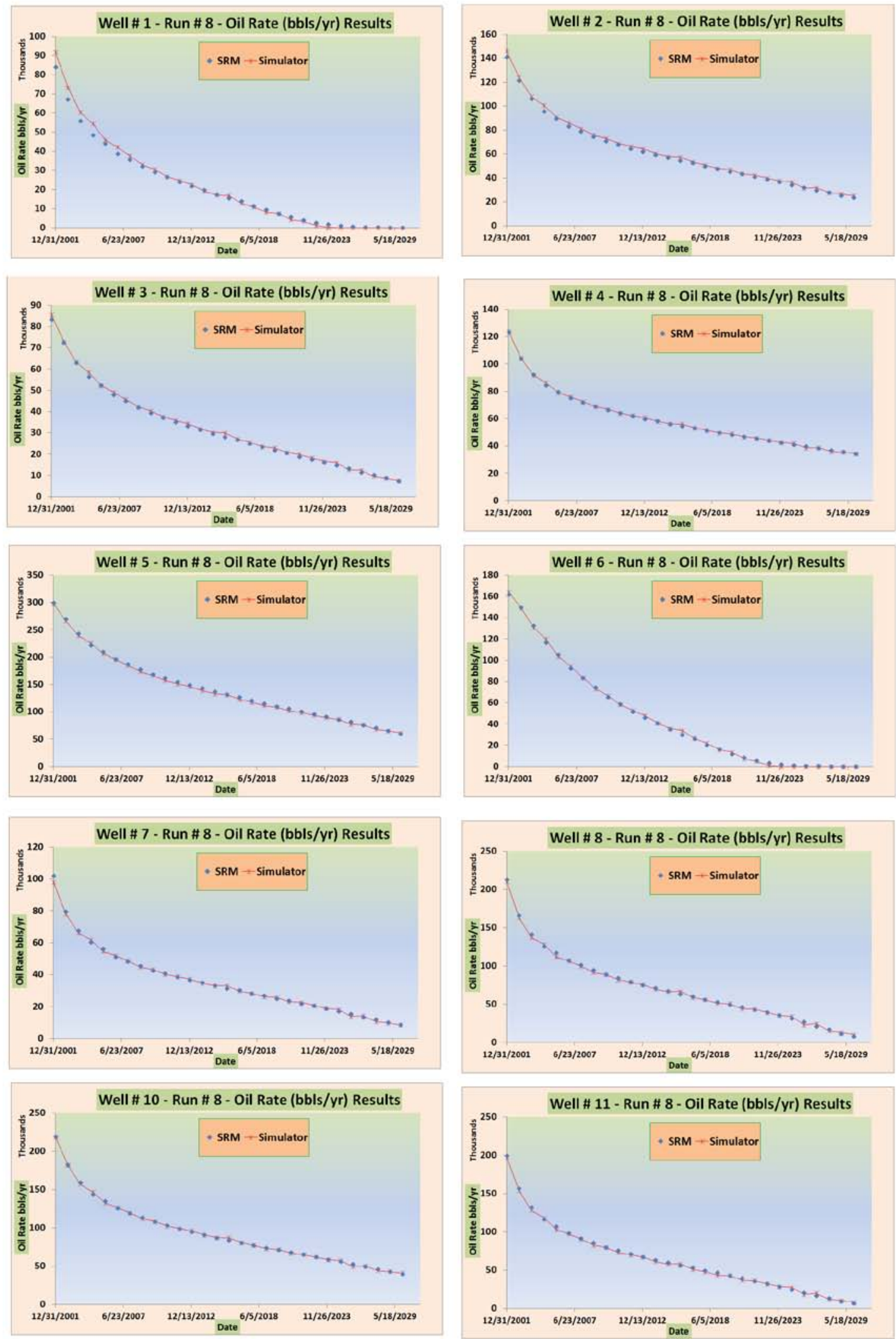

Figure 88- Comparison of the SRM results with simulator outputs after the training process- Run \# 8 

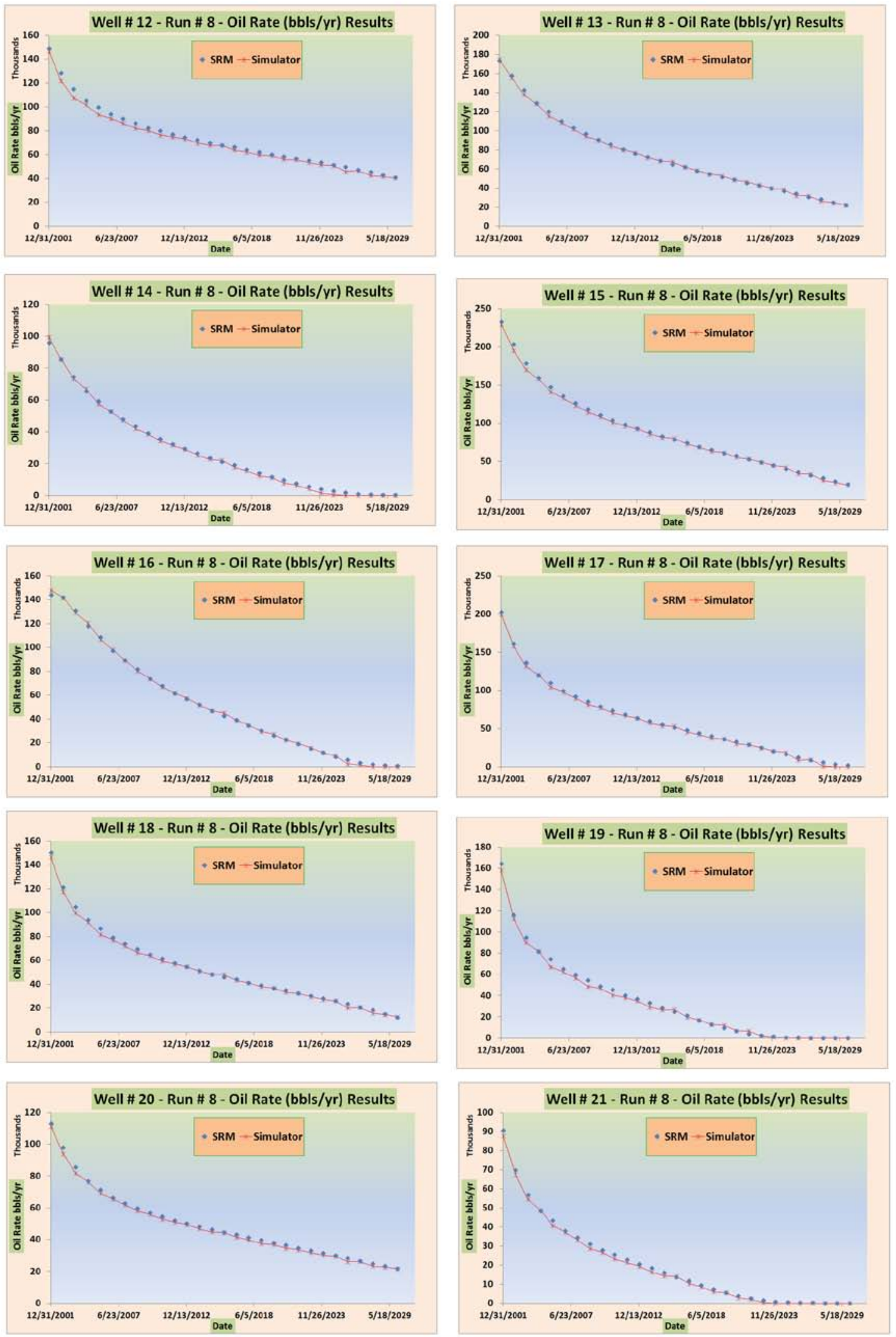

Figure 89- Comparison of the SRM results with simulator outputs after the training process- Run \# 8 

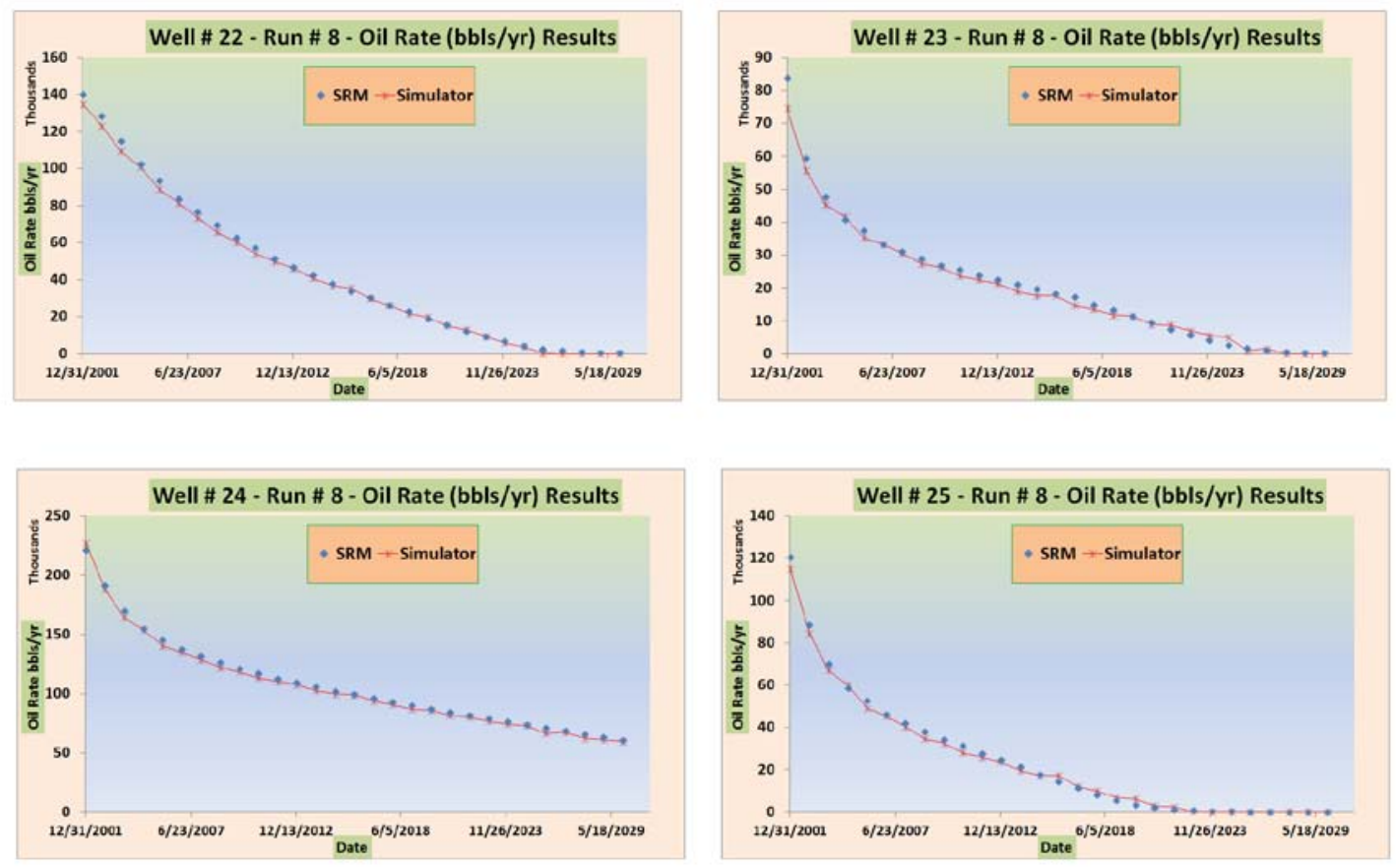

Figure 90- Comparison of the SRM results with simulator outputs after the training process- Run \# 8

\section{a.9 - Realization \# 9:}
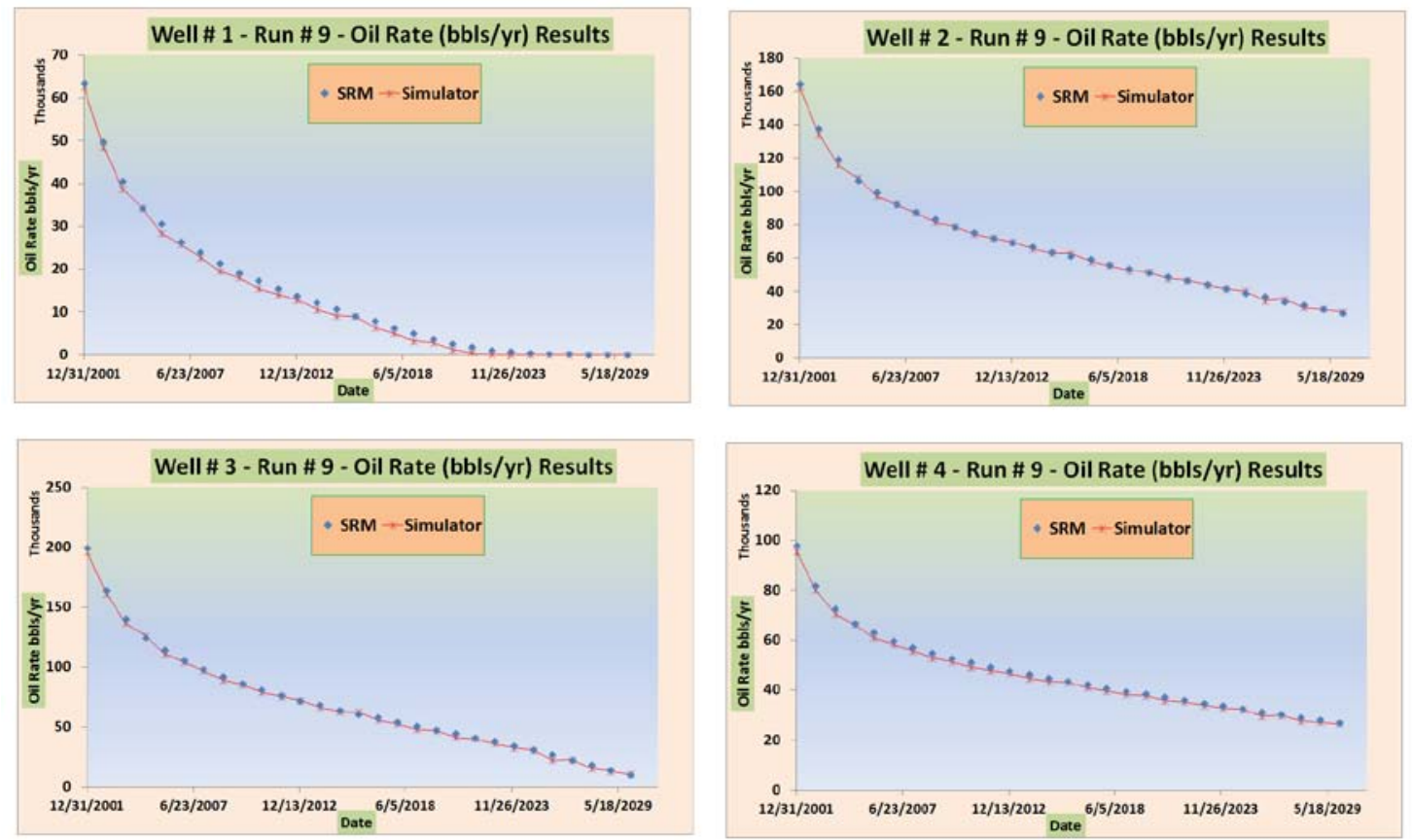

Figure 91- Comparison of the SRM results with simulator outputs after the training process- Run \# 9 

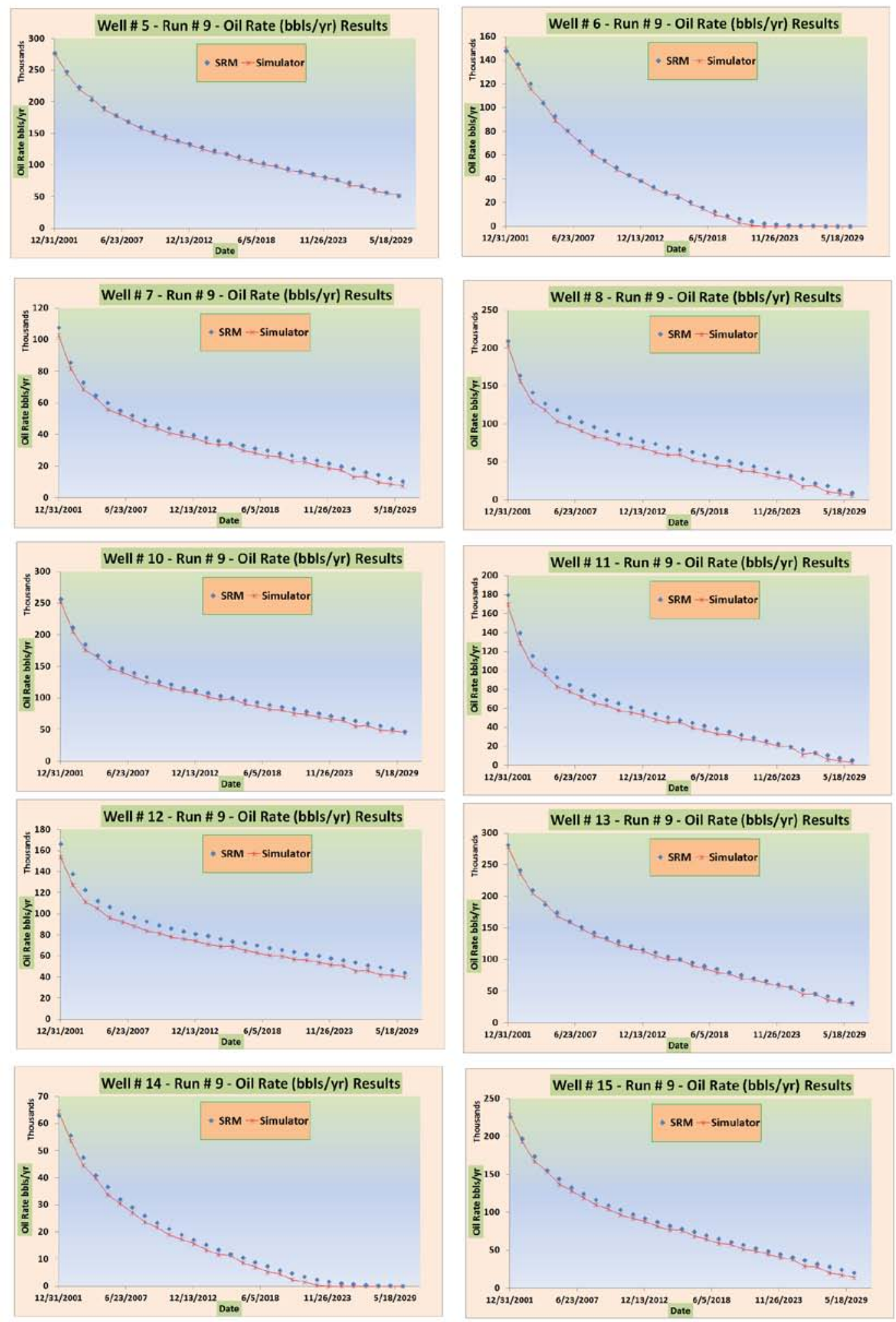

Figure 92- Comparison of the SRM results with simulator outputs after the training process- Run \# 9 

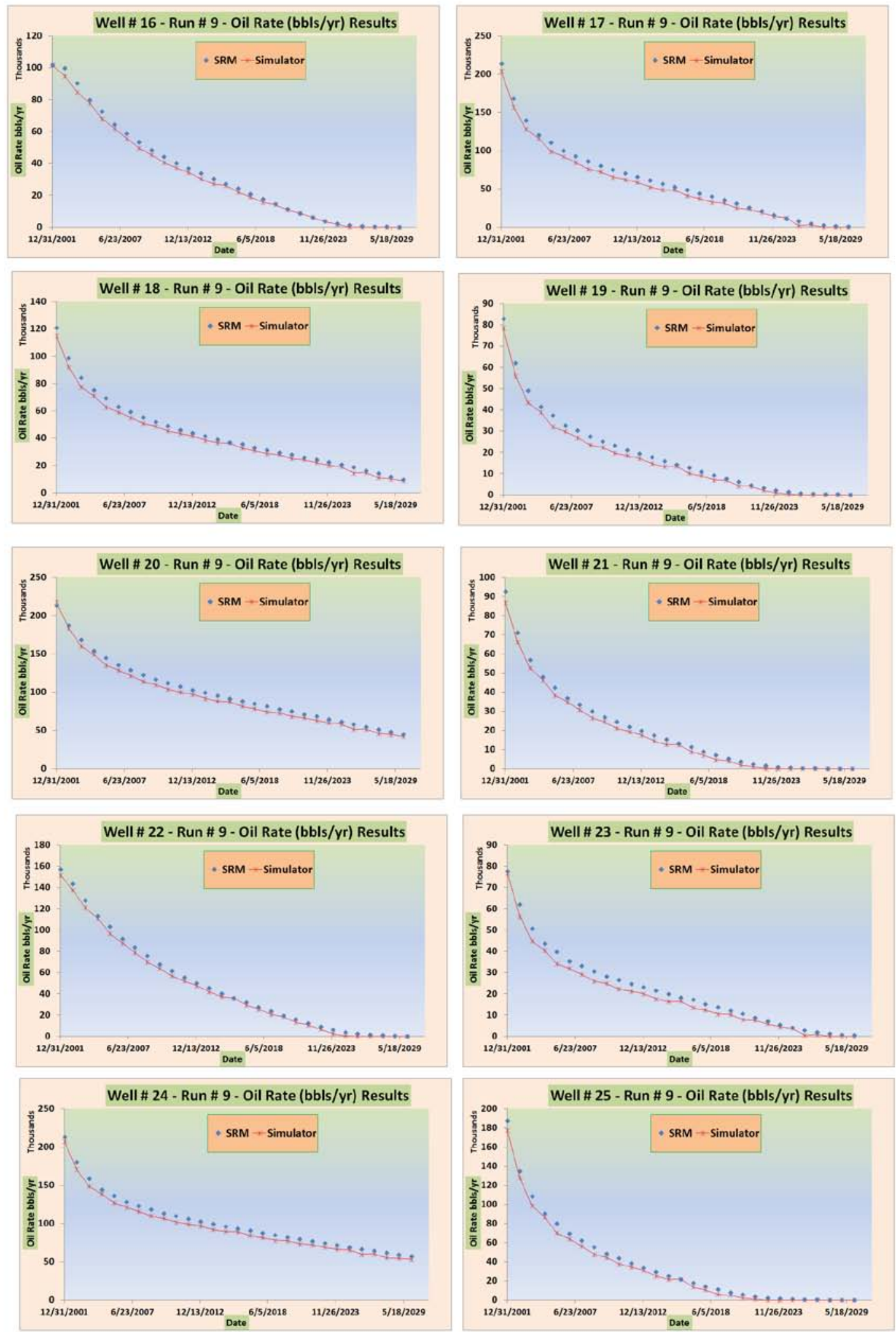

Figure 93- Comparison of the SRM results with simulator outputs after the training process- Run \# 9 


\section{a.10 - Realization \# 10:}
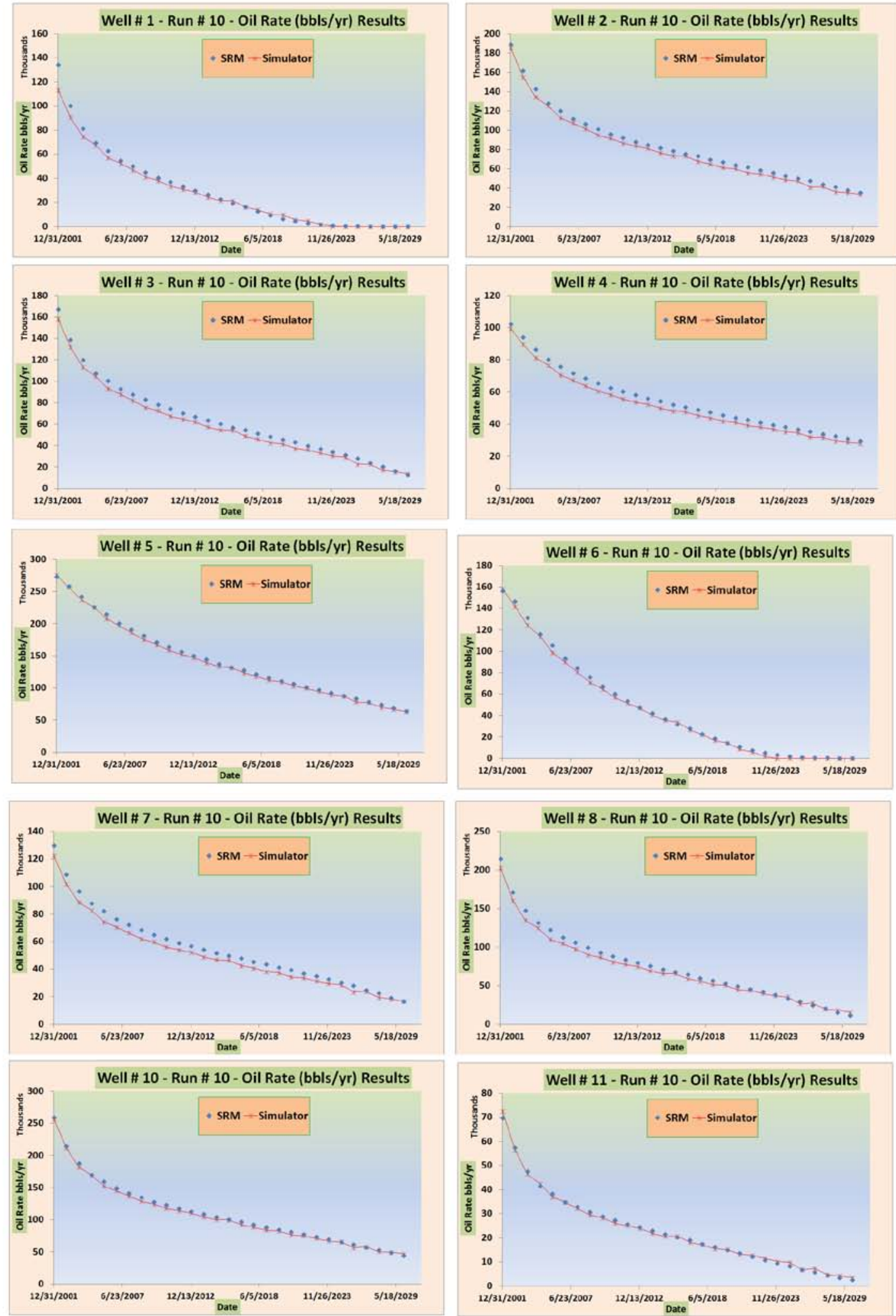

Figure 94- Comparison of the SRM results with simulator outputs after the training process- Run \# 10 

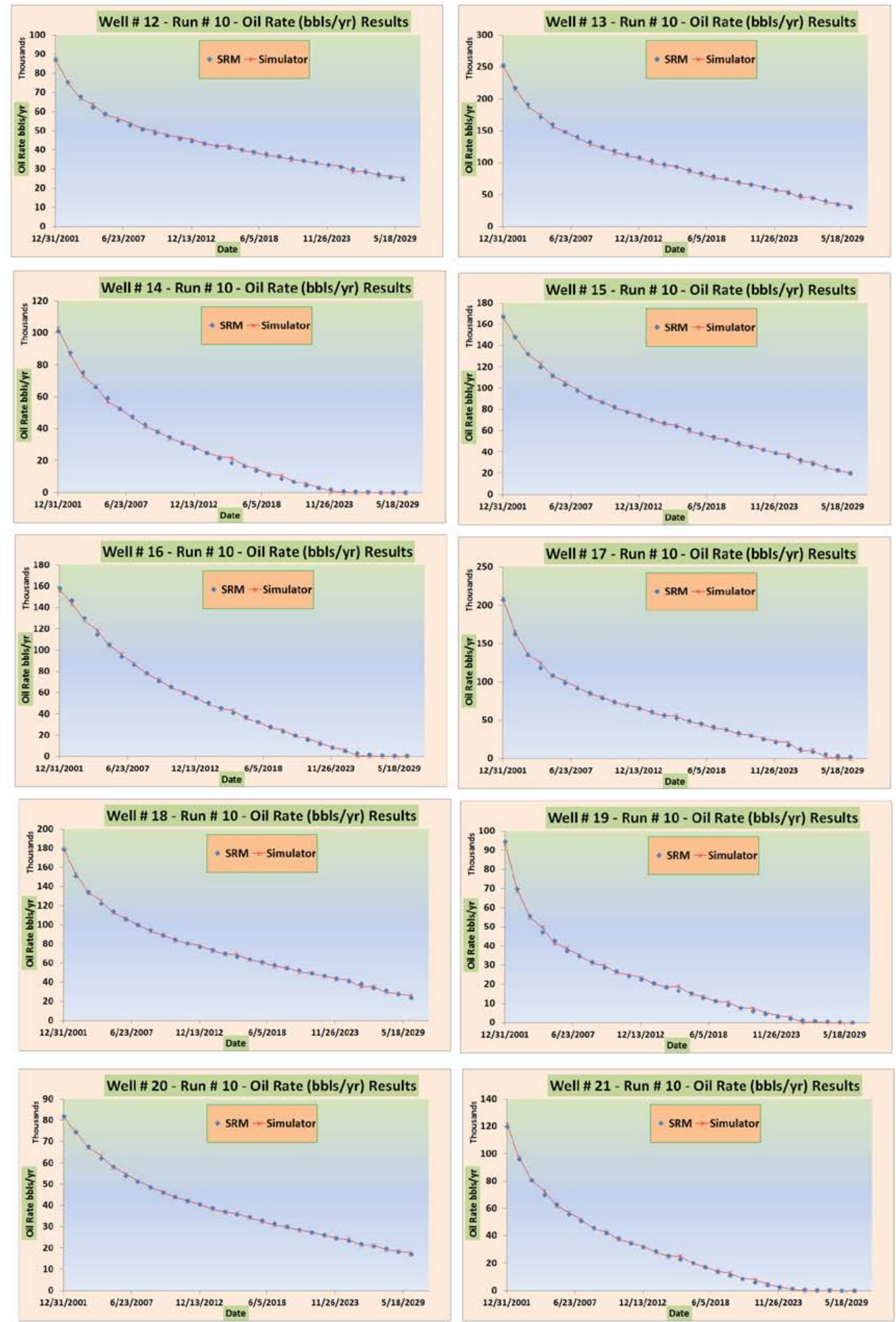

Figure 95- Comparison of the SRM results with simulator outputs after the training process- Run \# 10 

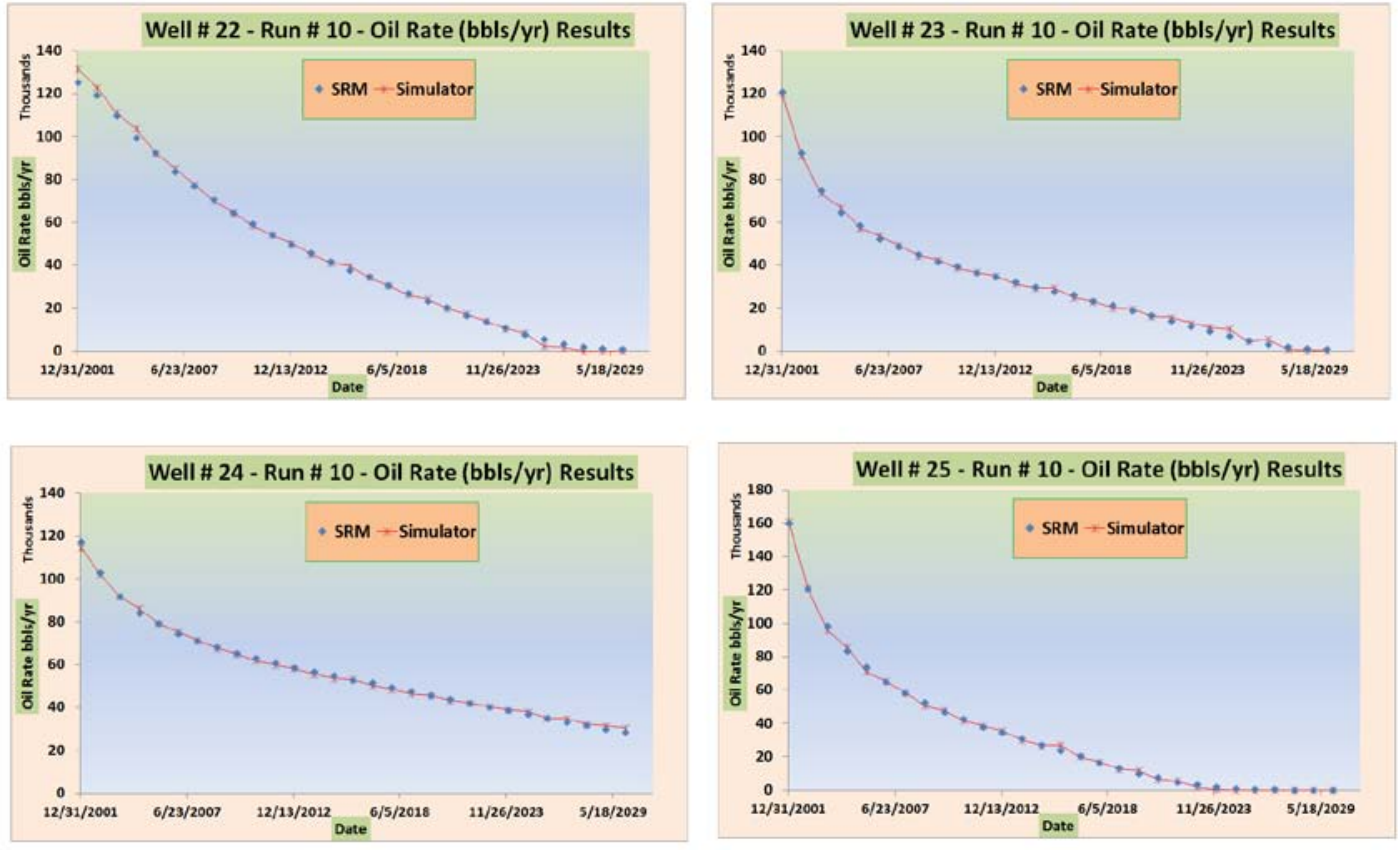

Figure 96- Comparison of the SRM results with simulator outputs after the training process- Run \# 10 


\section{b. Validation of SRM using blind verification realization}
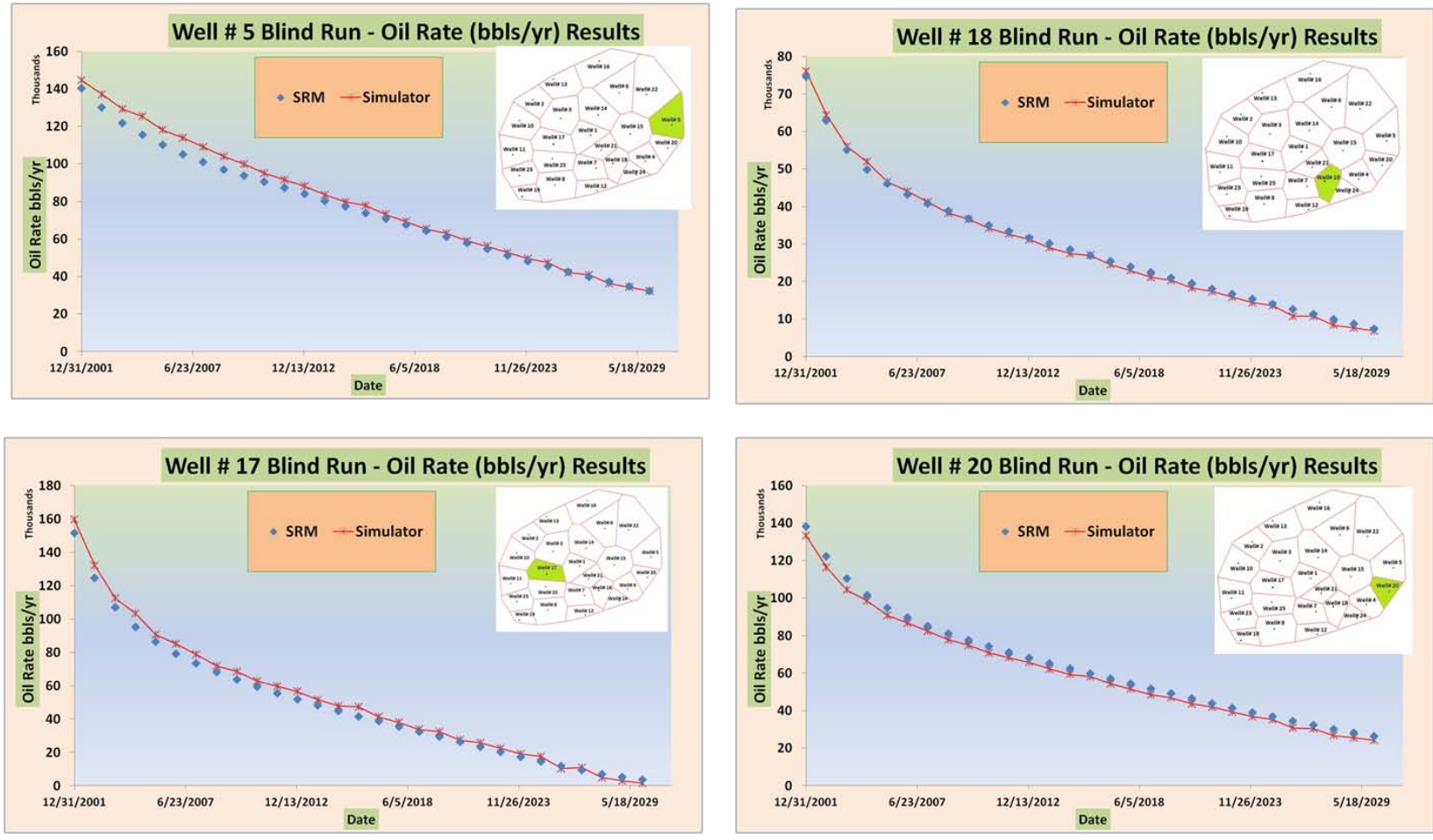

Figure 97- Comparison of the SRM results with simulator outputs, the blind verification run, wells 5, 17, 18 and 20
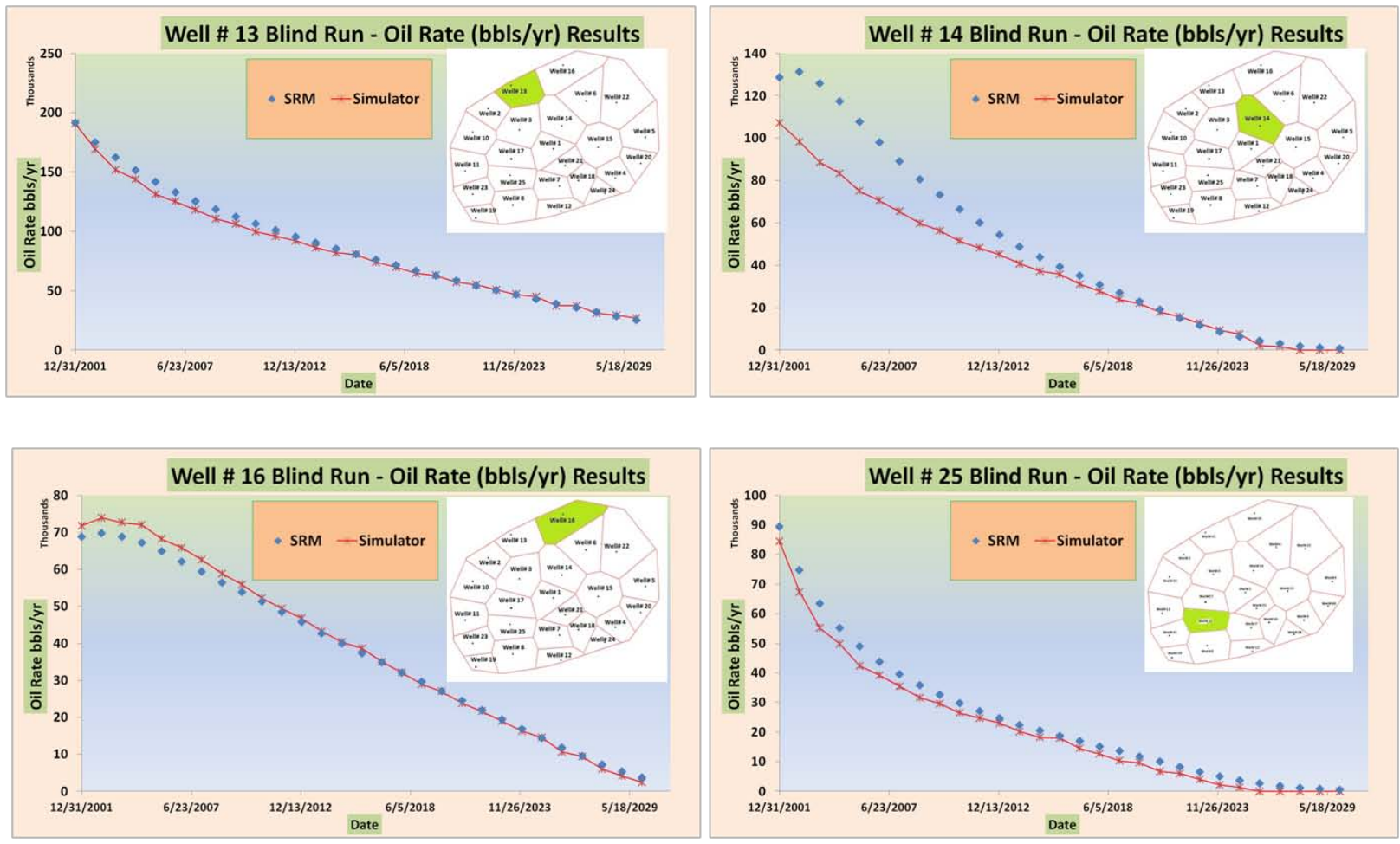

Figure 98- Comparison of the SRM results with simulator outputs, the blind verification run, wells 13, 14, 16 and 25 

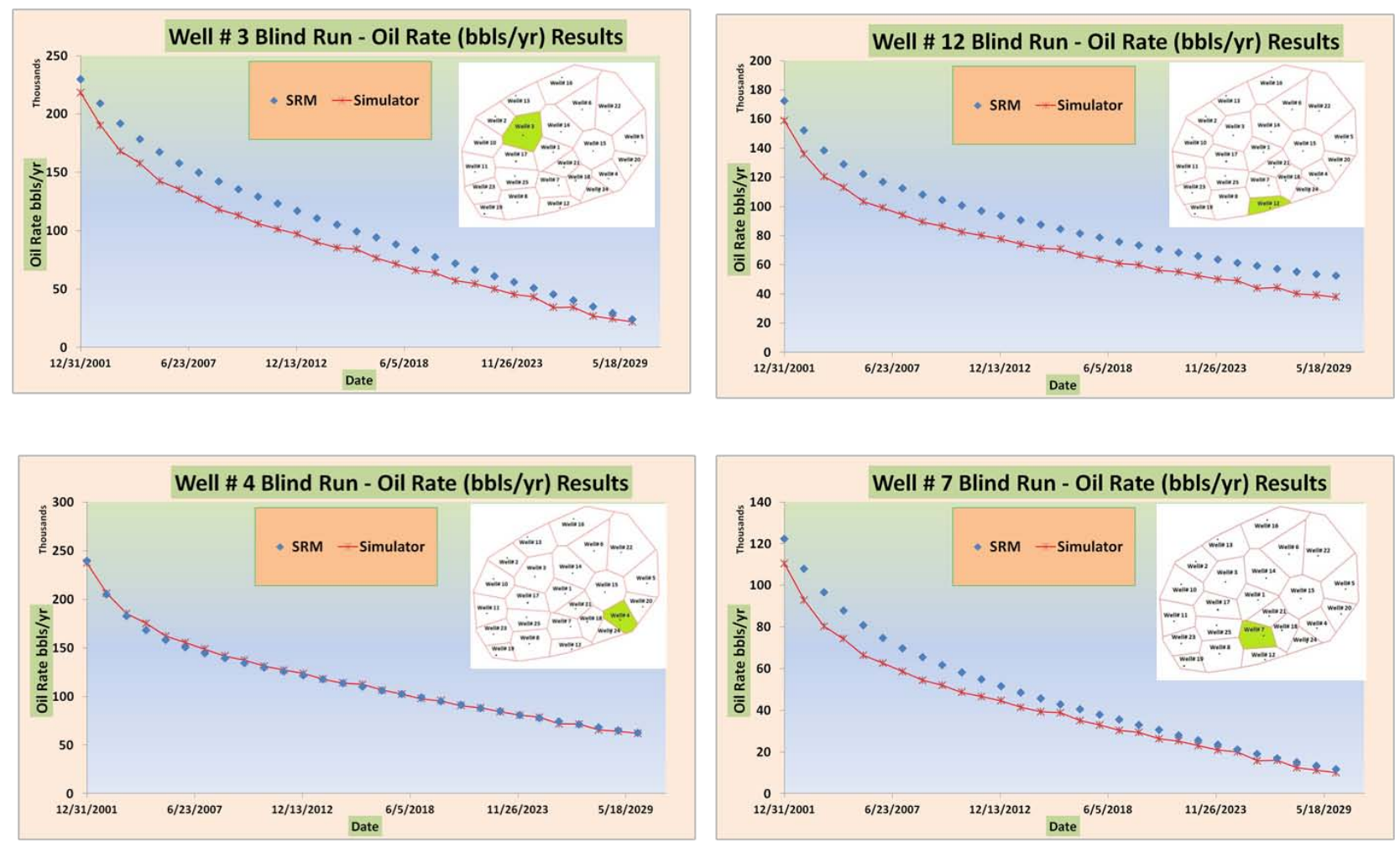

Figure 99- Comparison of the SRM results with simulator outputs, the blind verification run, wells 3, 4, 7 and 12
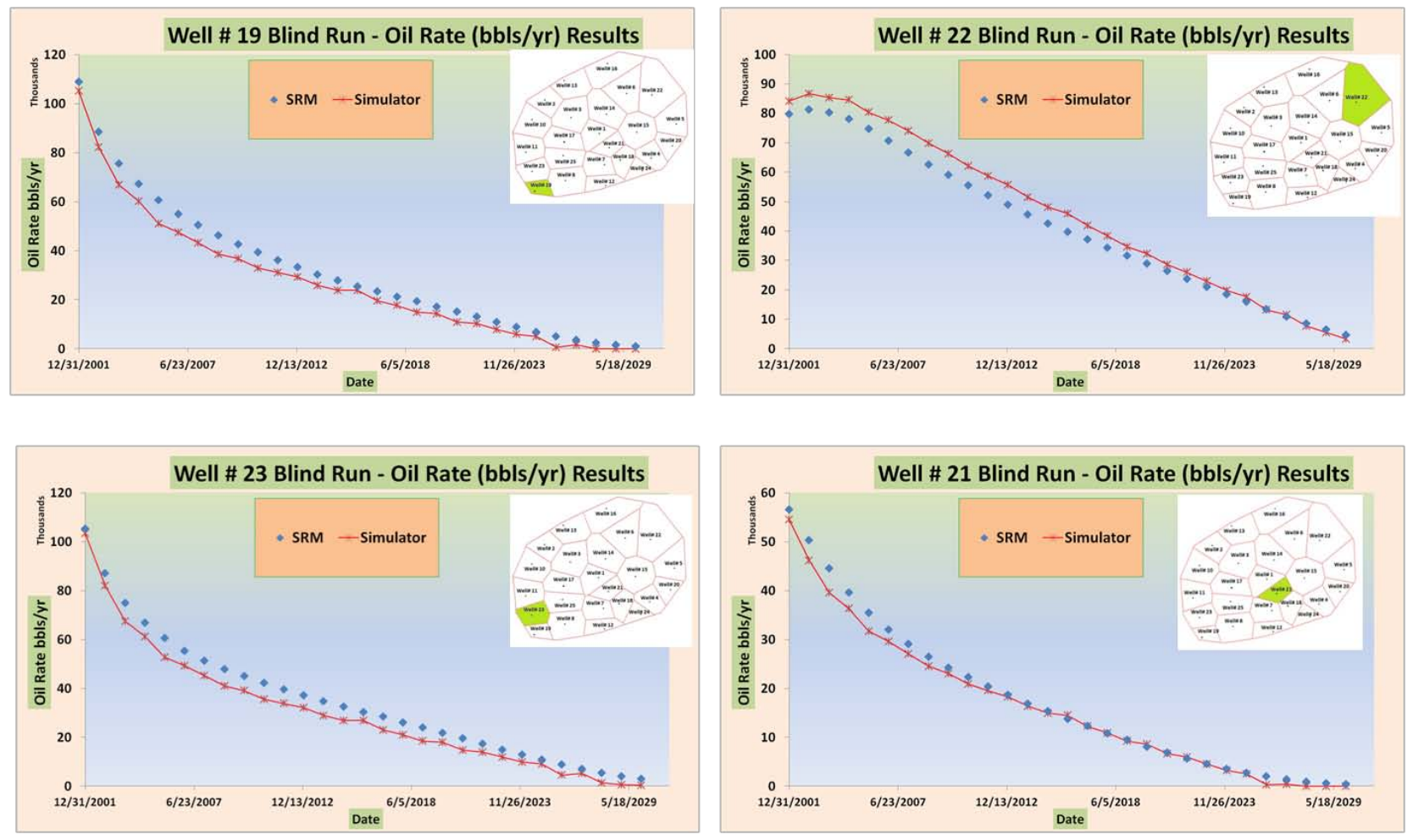

Figure 100- Comparison of the SRM results with simulator outputs, the blind verification run, wells 19, 21, 22 and 23 

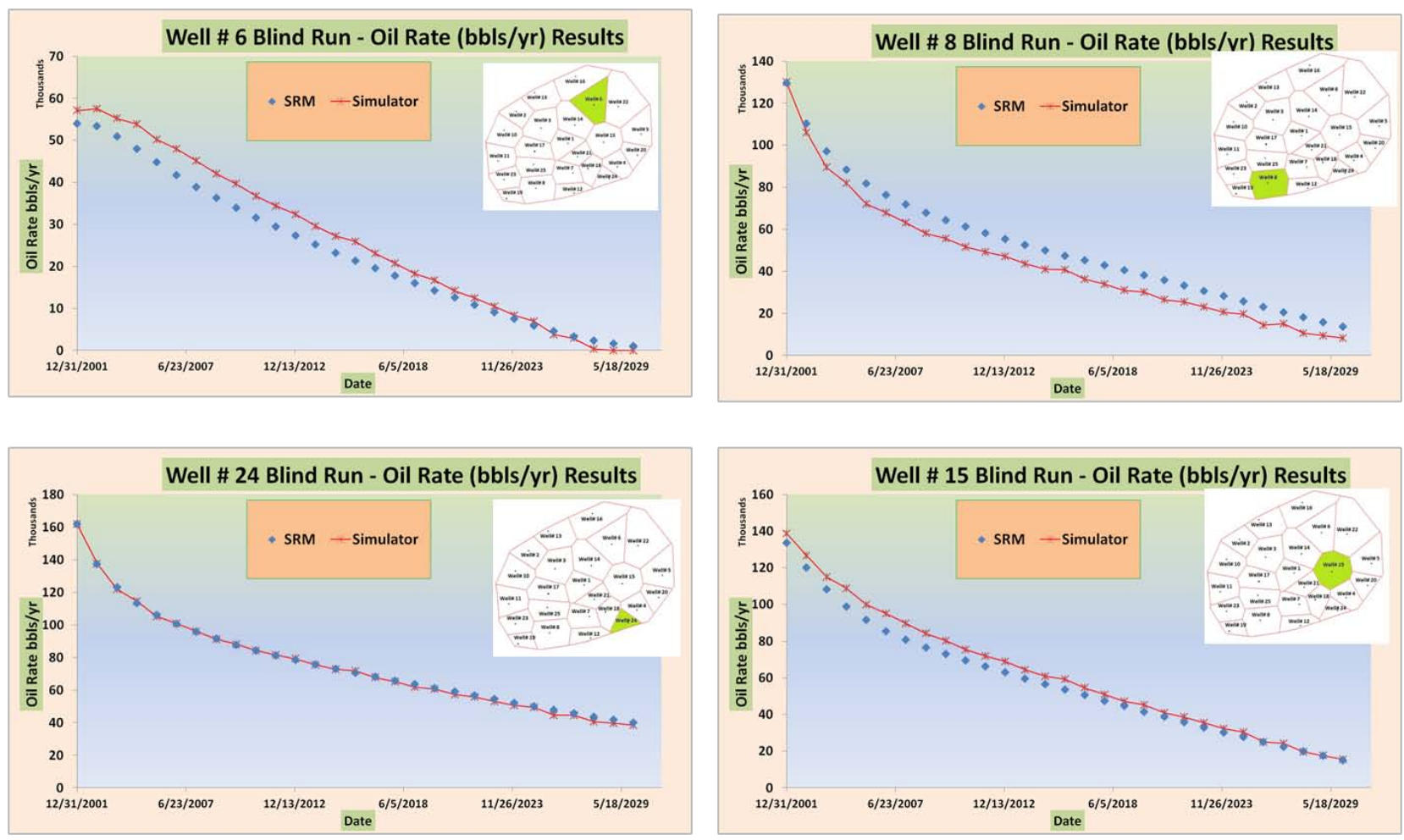

Figure 101- Comparison of the SRM results with simulator outputs, the blind verification run, wells 6, 8, 15 and 24
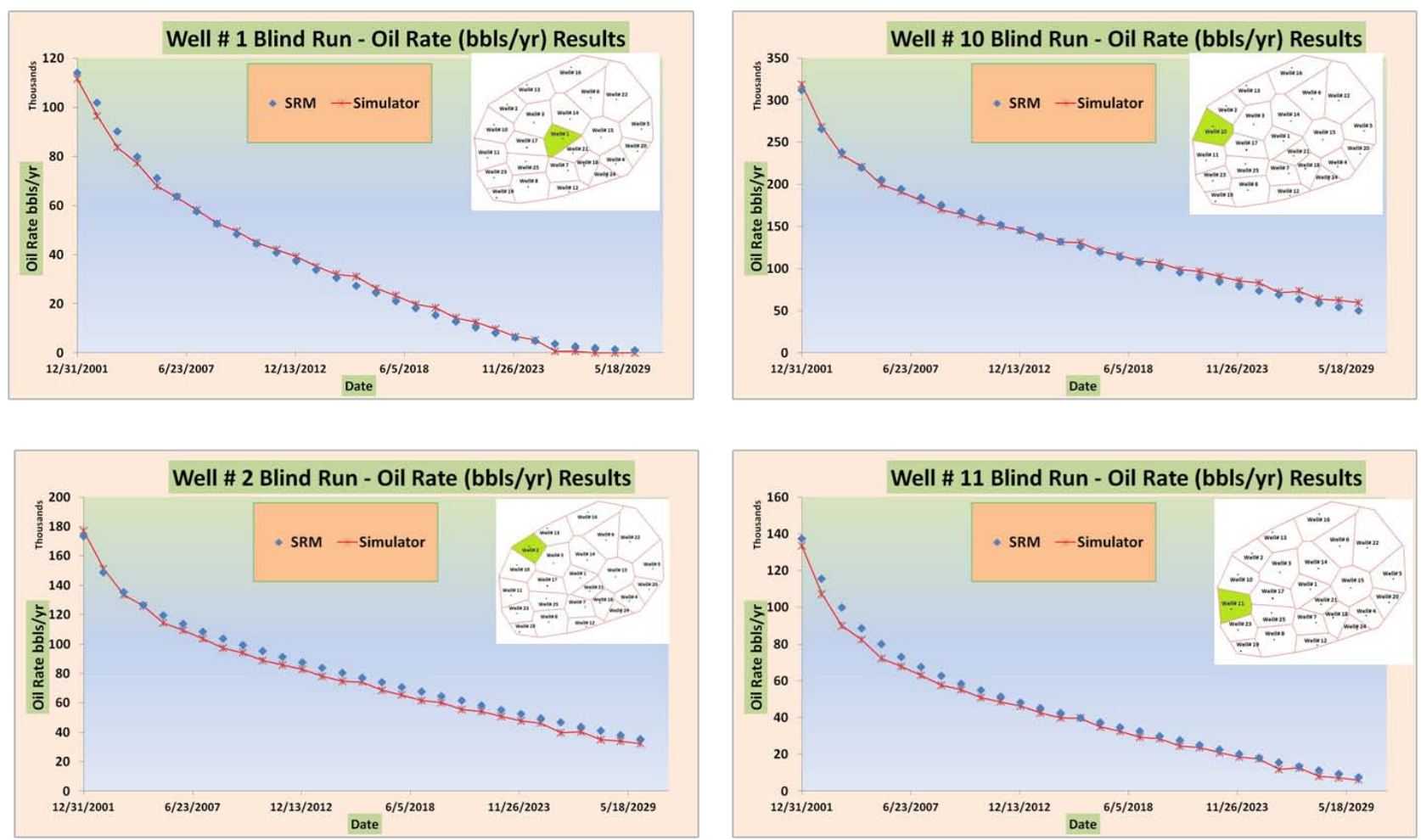

Figure 102- Comparison of the SRM results with simulator outputs, the blind verification run, wells 1, 2, 10 and 11 


\section{c. History match results}
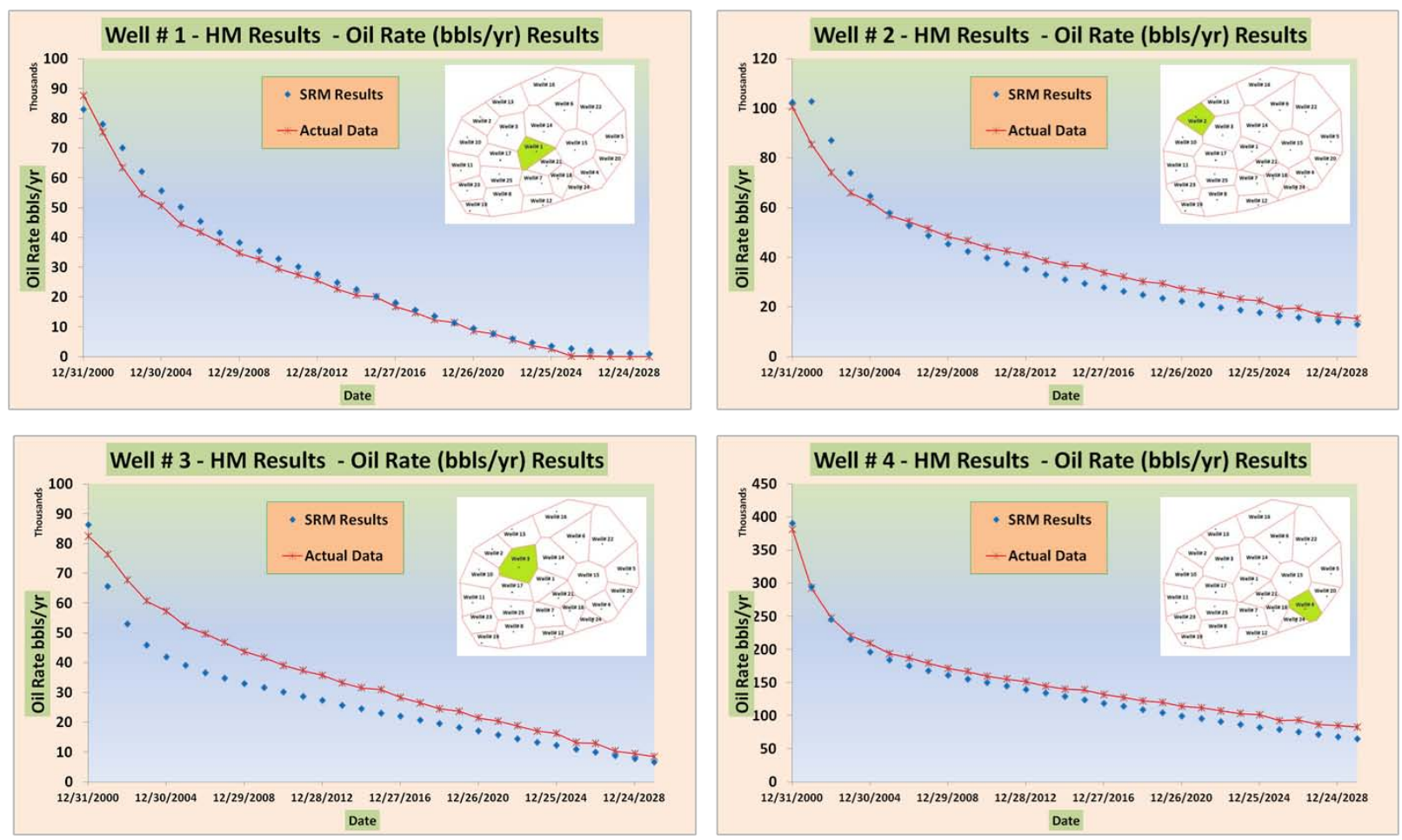

Figure 103- Comparison of the SRM results with field data, history match results; wells 1, 2, 3 and 4
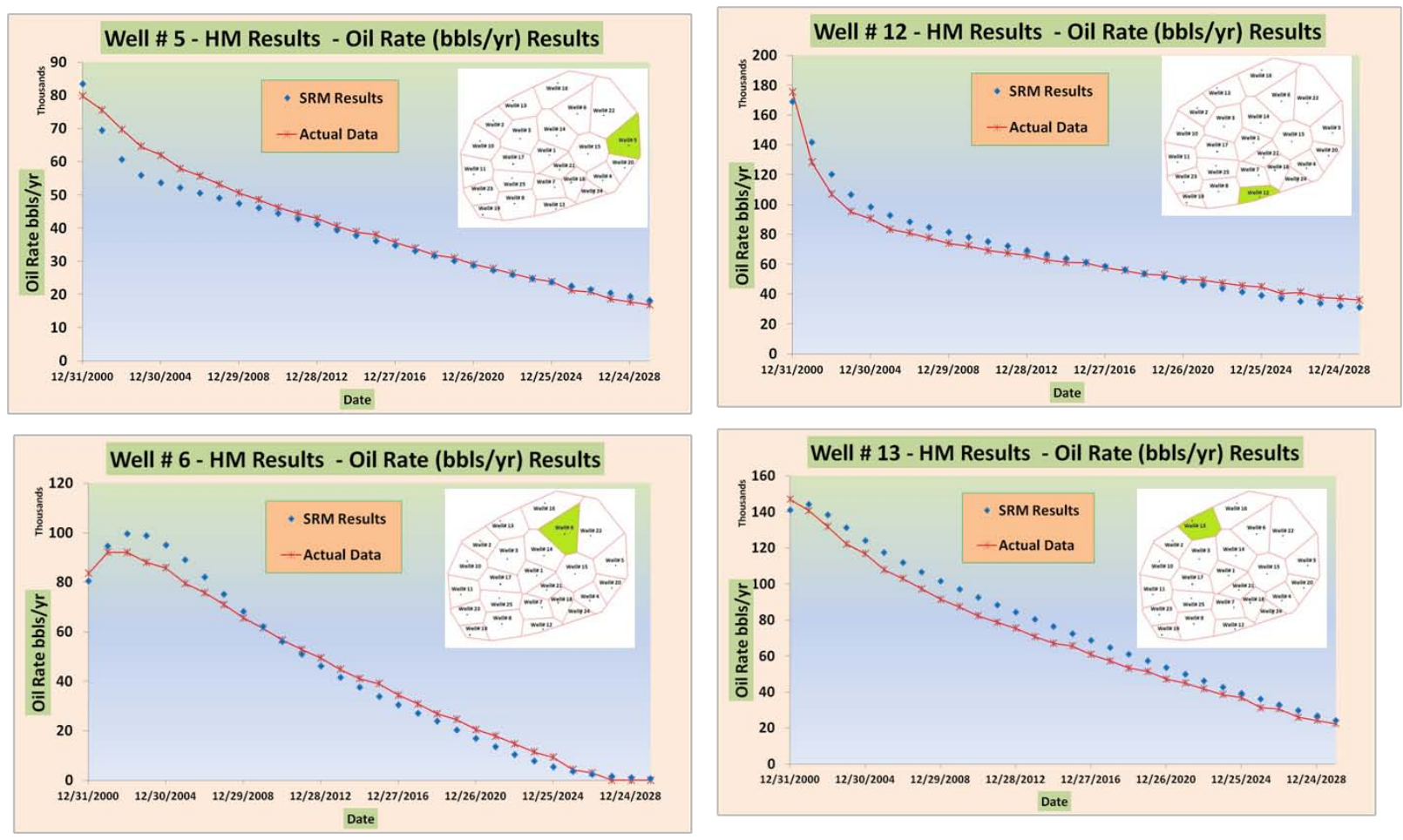

Figure 104- Comparison of the SRM results with field data, history match results; wells 5, 6, 12 and 13 

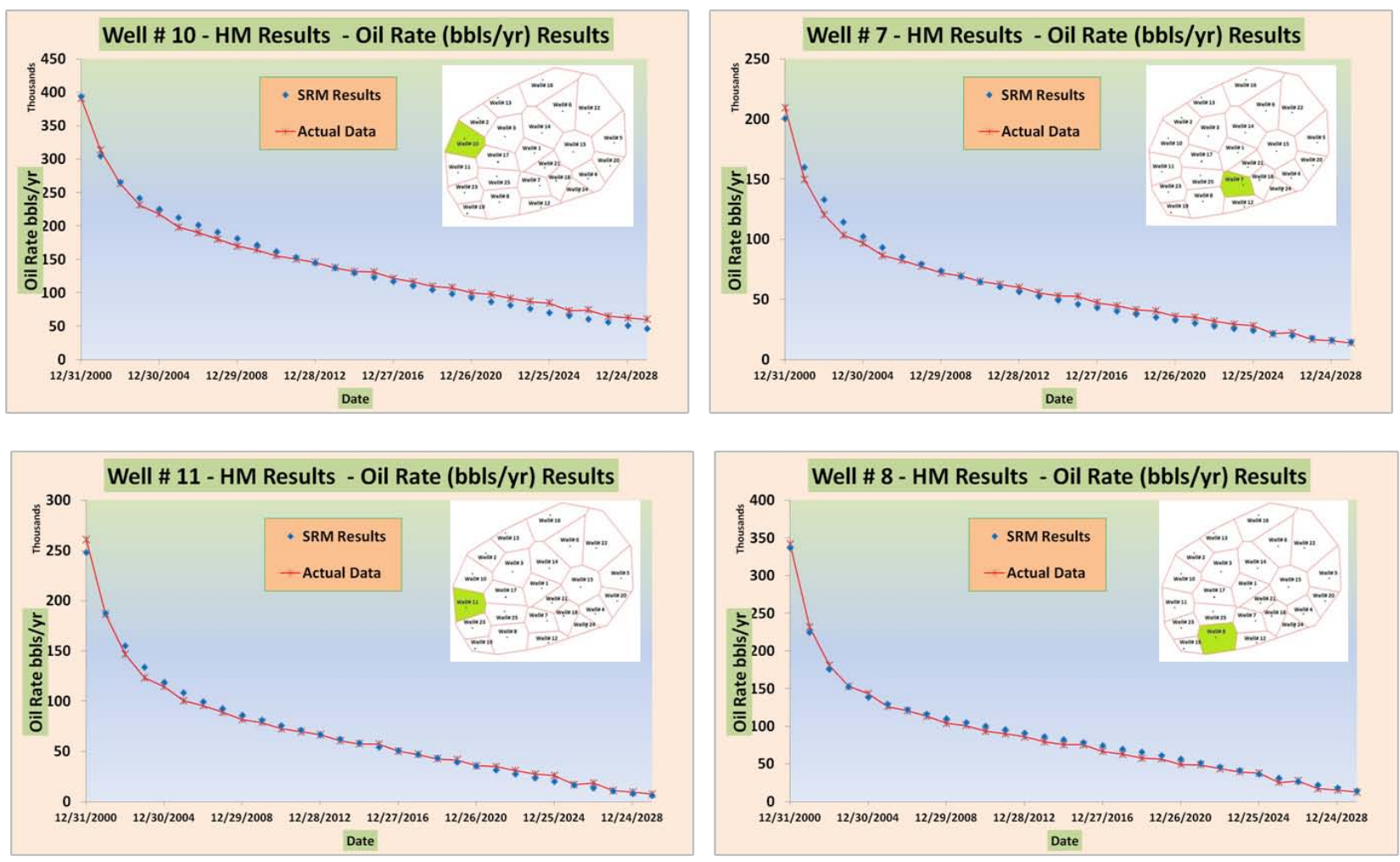

Figure 105- Comparison of the SRM results with field data, history match results; wells 7, 8, 10 and 11
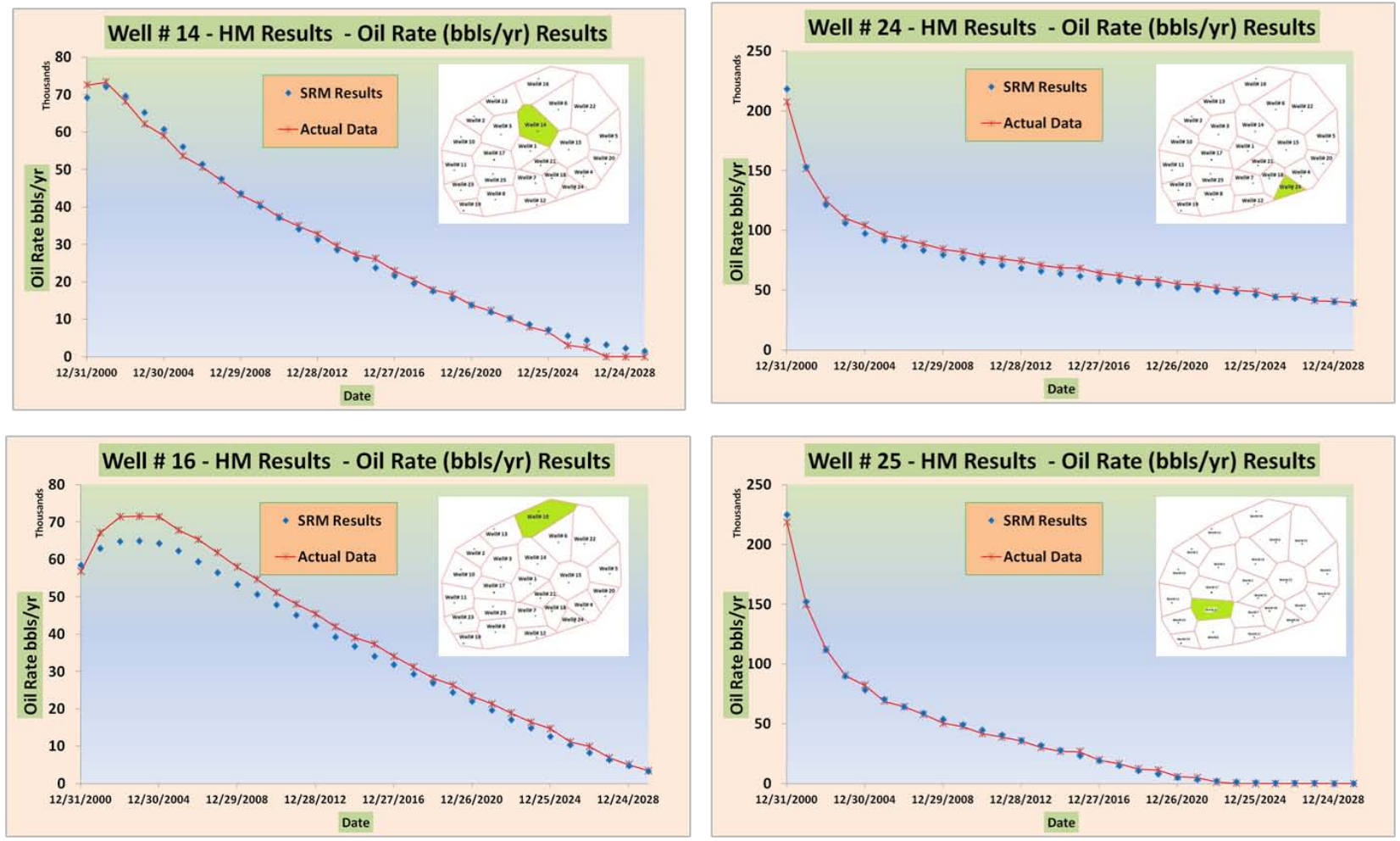

Figure 106- Comparison of the SRM results with field data, history match results; wells 14, 16, 24 and 25 

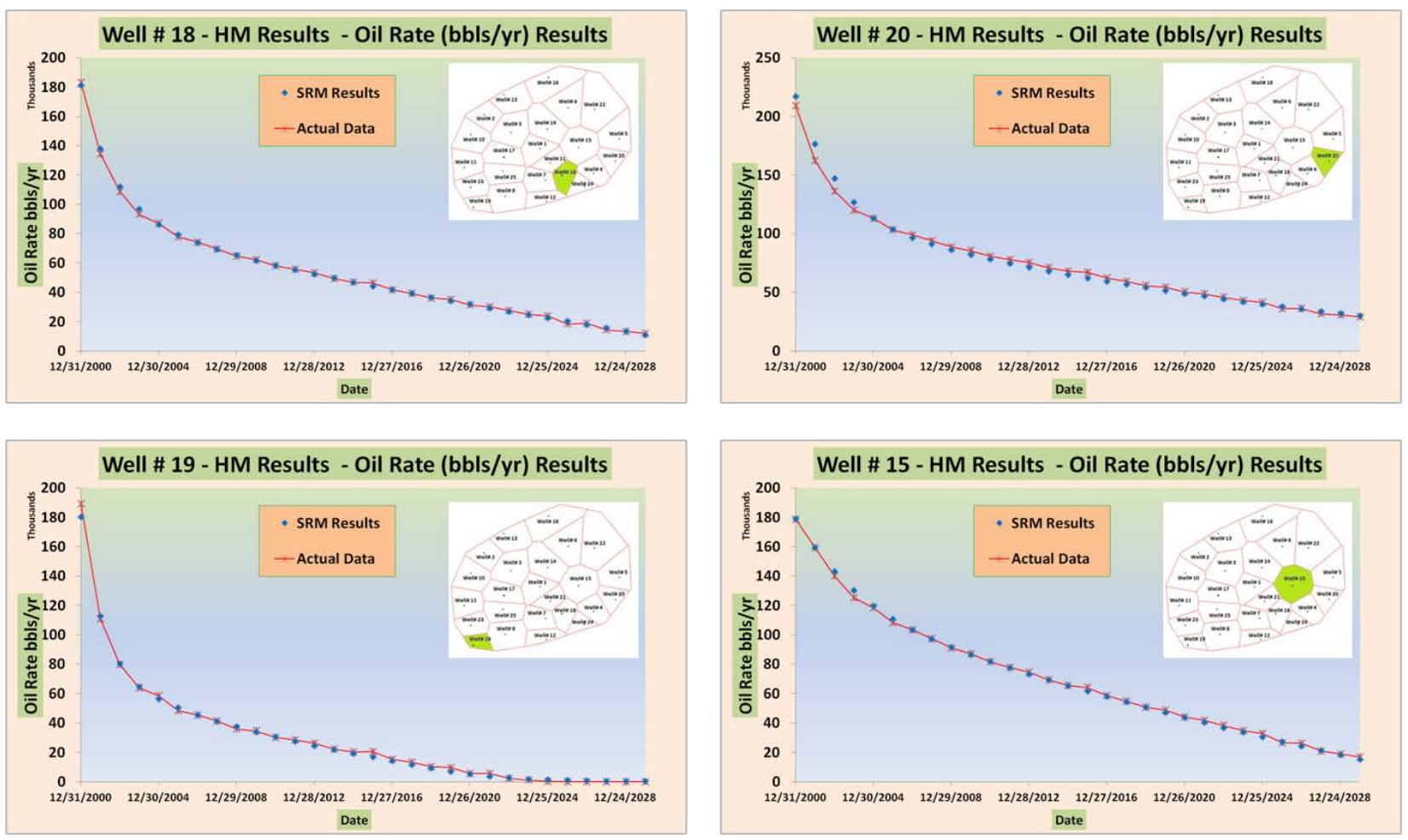

Figure 107- Comparison of the SRM results with field data, history match results; wells 15, 18, 19 and 20
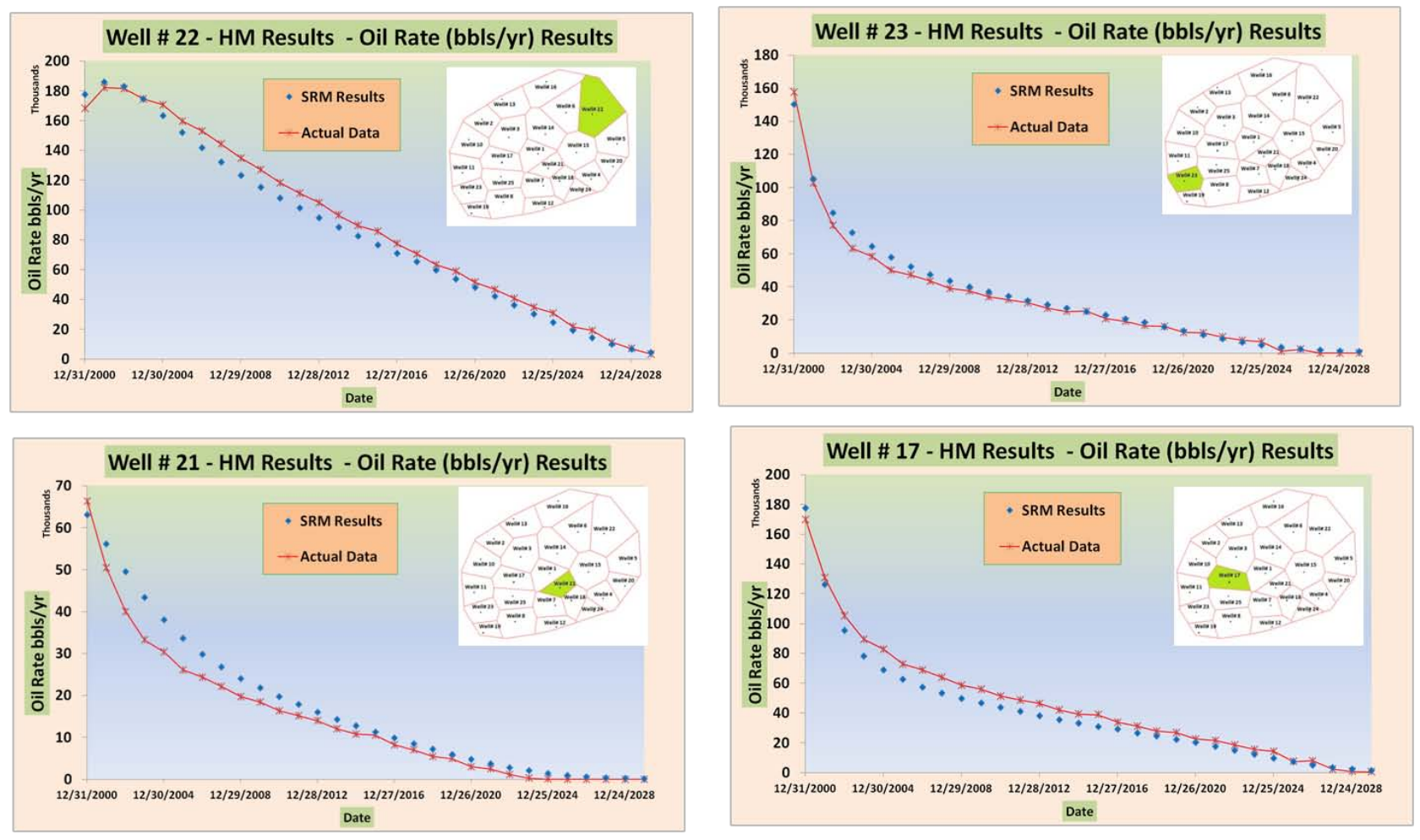

Figure 108- Comparison of the SRM results with field data, history match results; wells 17, 21, 22 and 23 


\section{d. Results of simulator using matched permeability distribution}

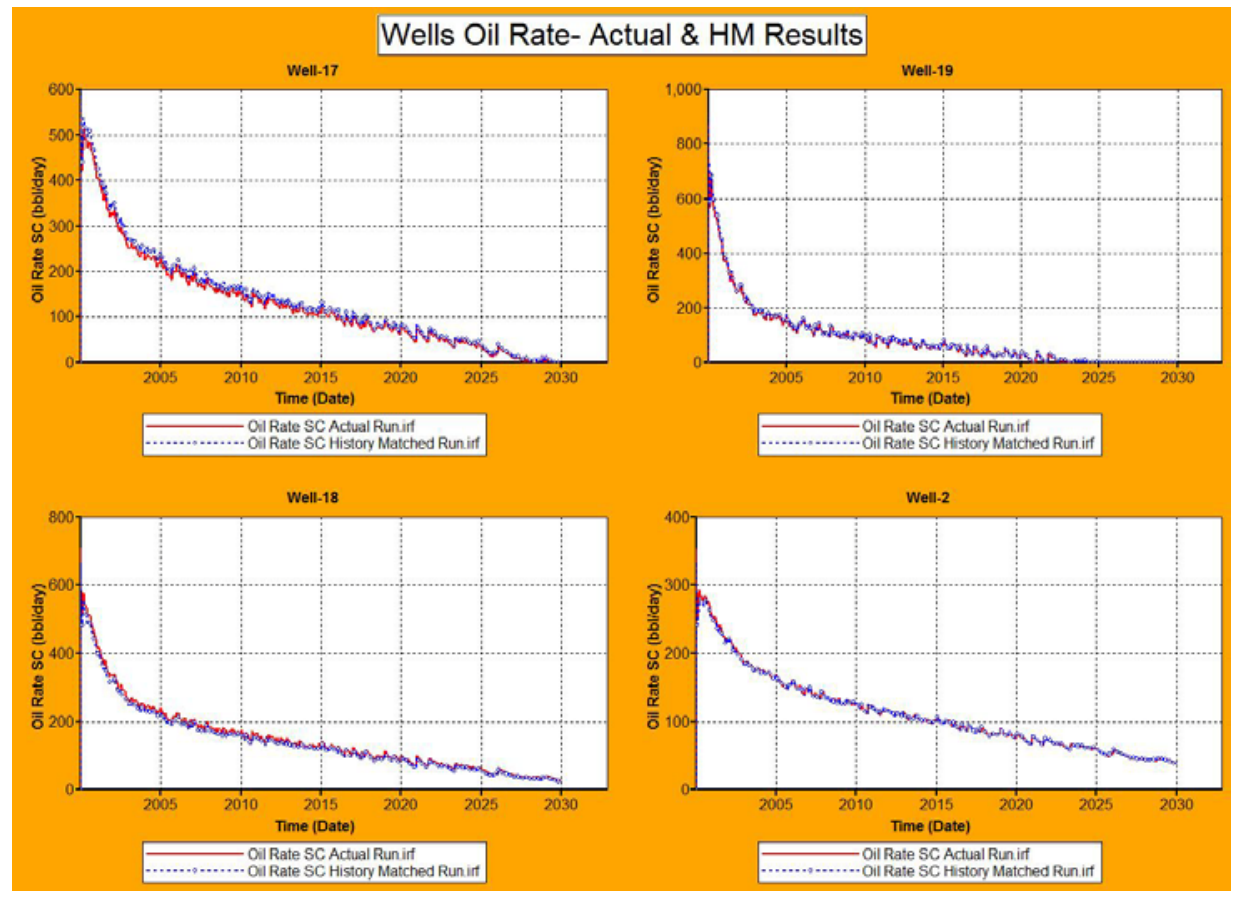

Figure 109- Comparison of oil rate production between actual data and the results coming from simulator after applying the matched permeability map from SRM

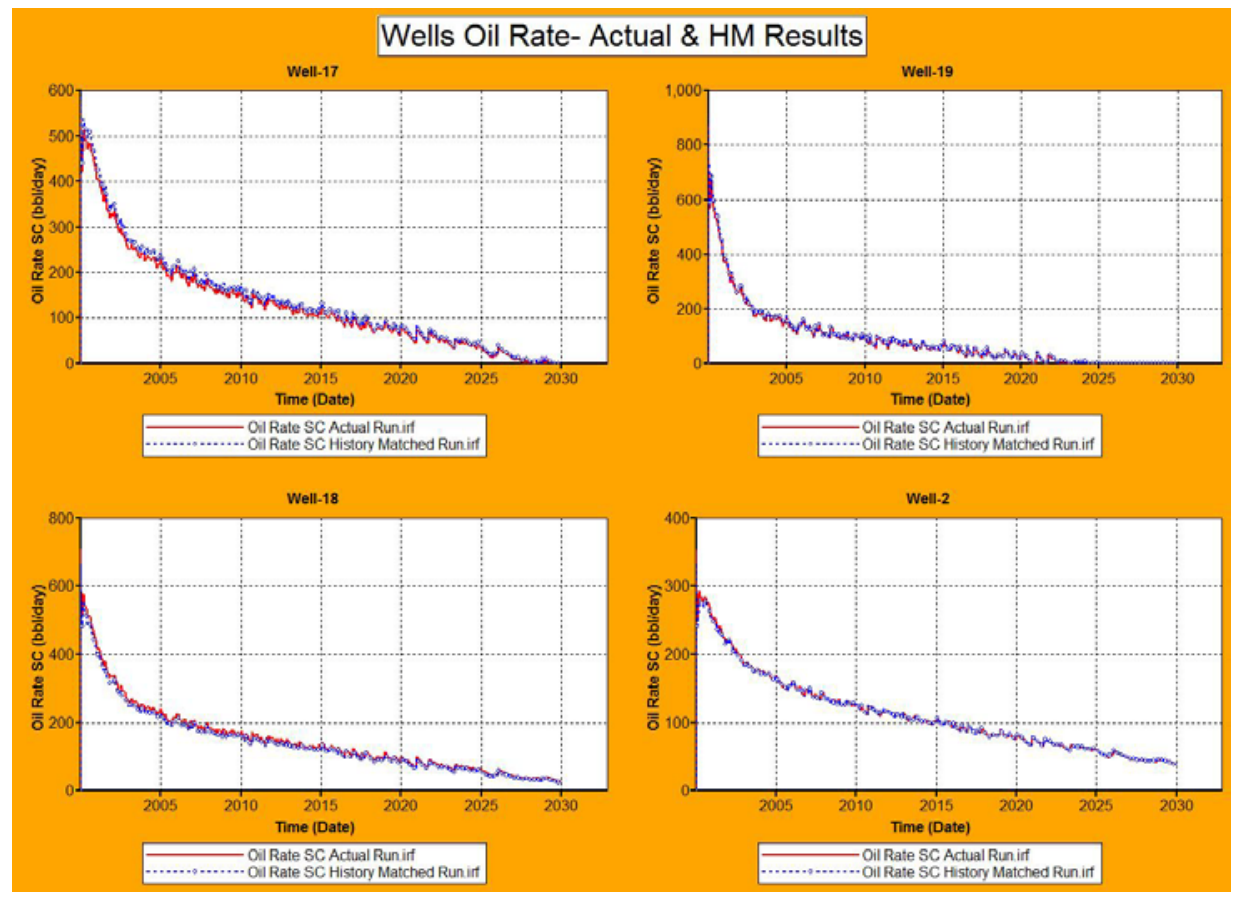

Figure 110-Comparison of oil rate production between actual data and the results coming from simulator after applying the matched permeability map from SRM 


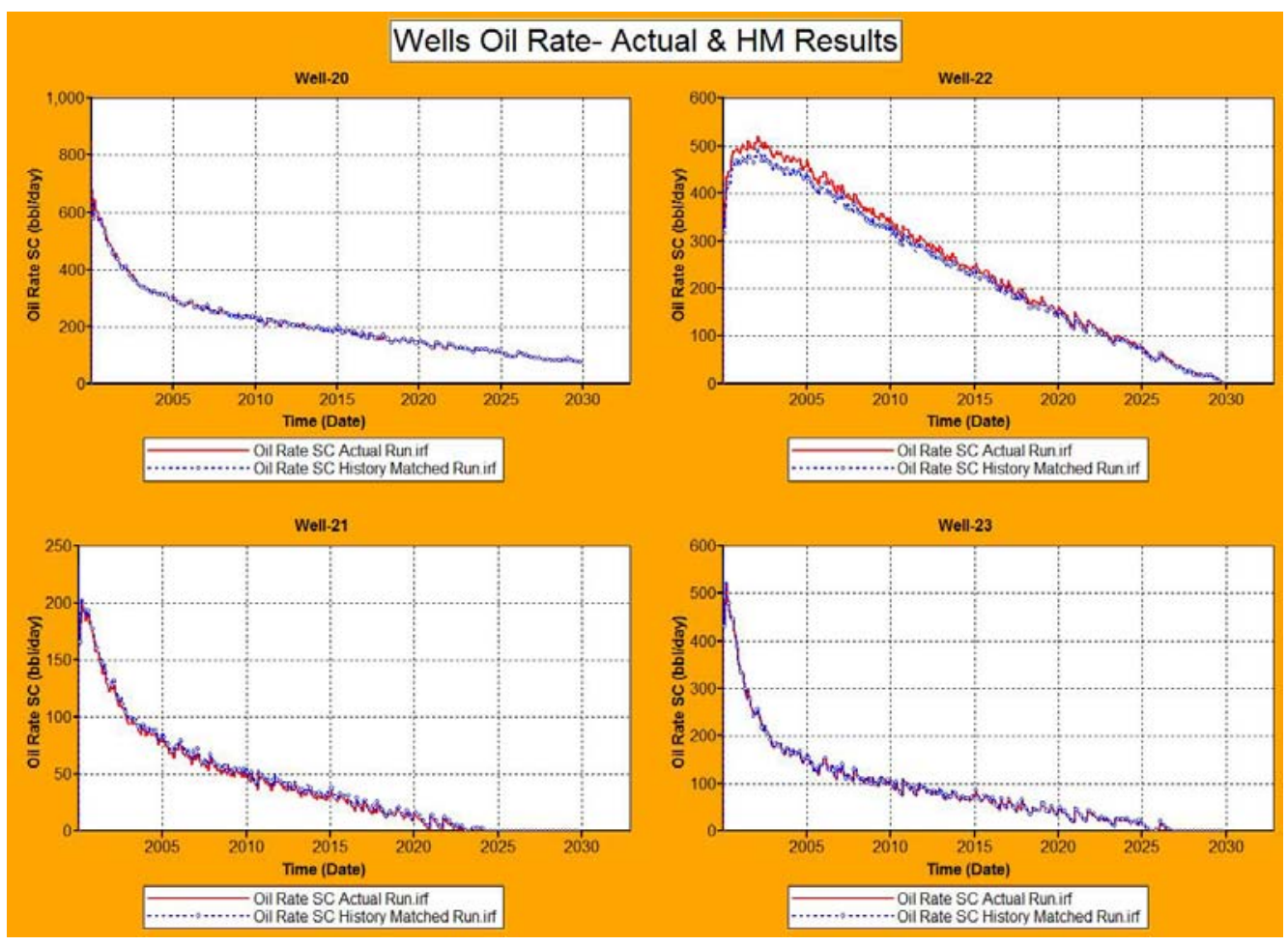

Figure 111- Comparison of oil rate production between actual data and the results coming from simulator after applying the matched permeability map from SRM

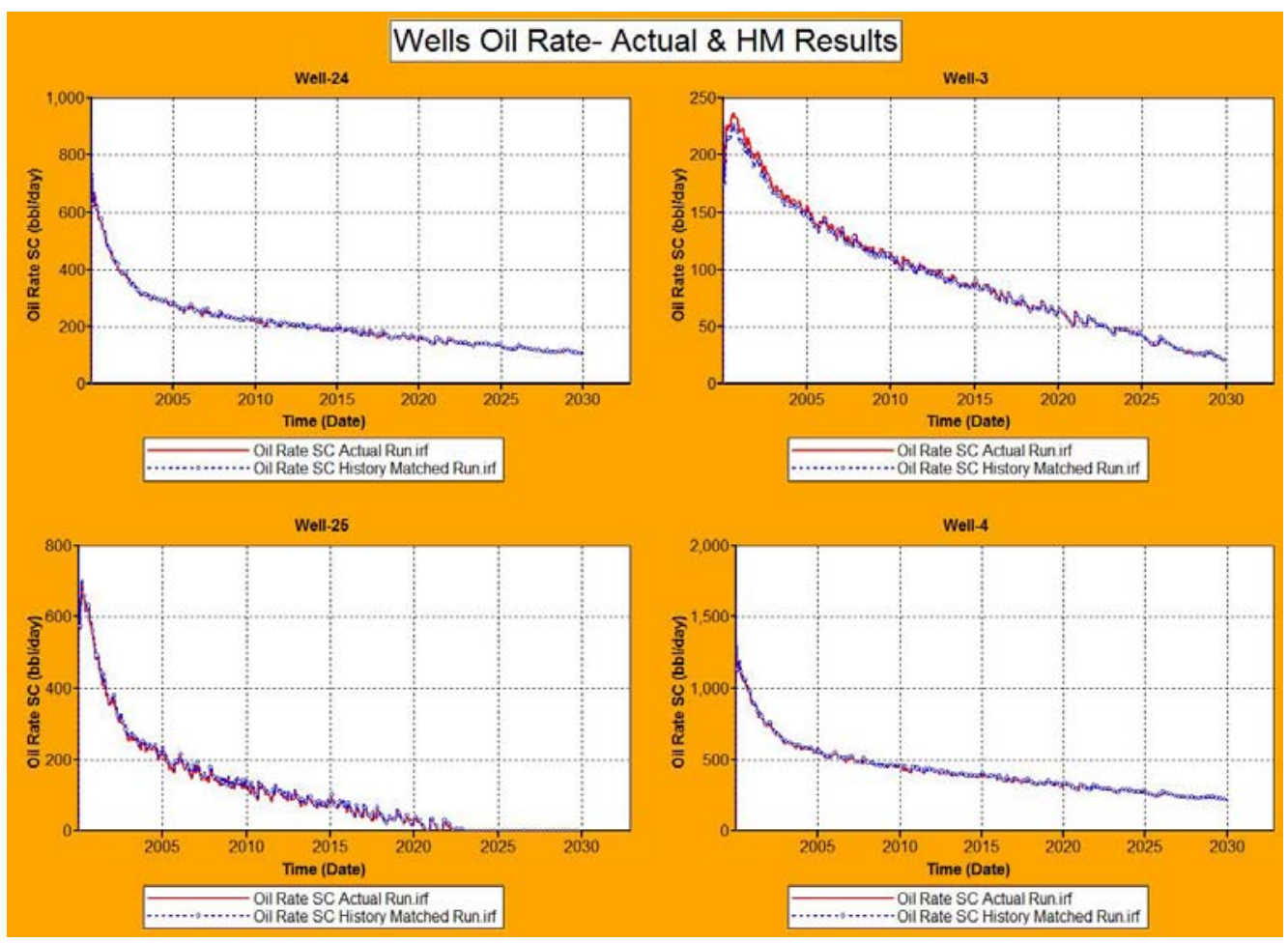

Figure 112- Comparison of oil rate production between actual data and the results coming from simulator after applying the matched permeability map from SRM 


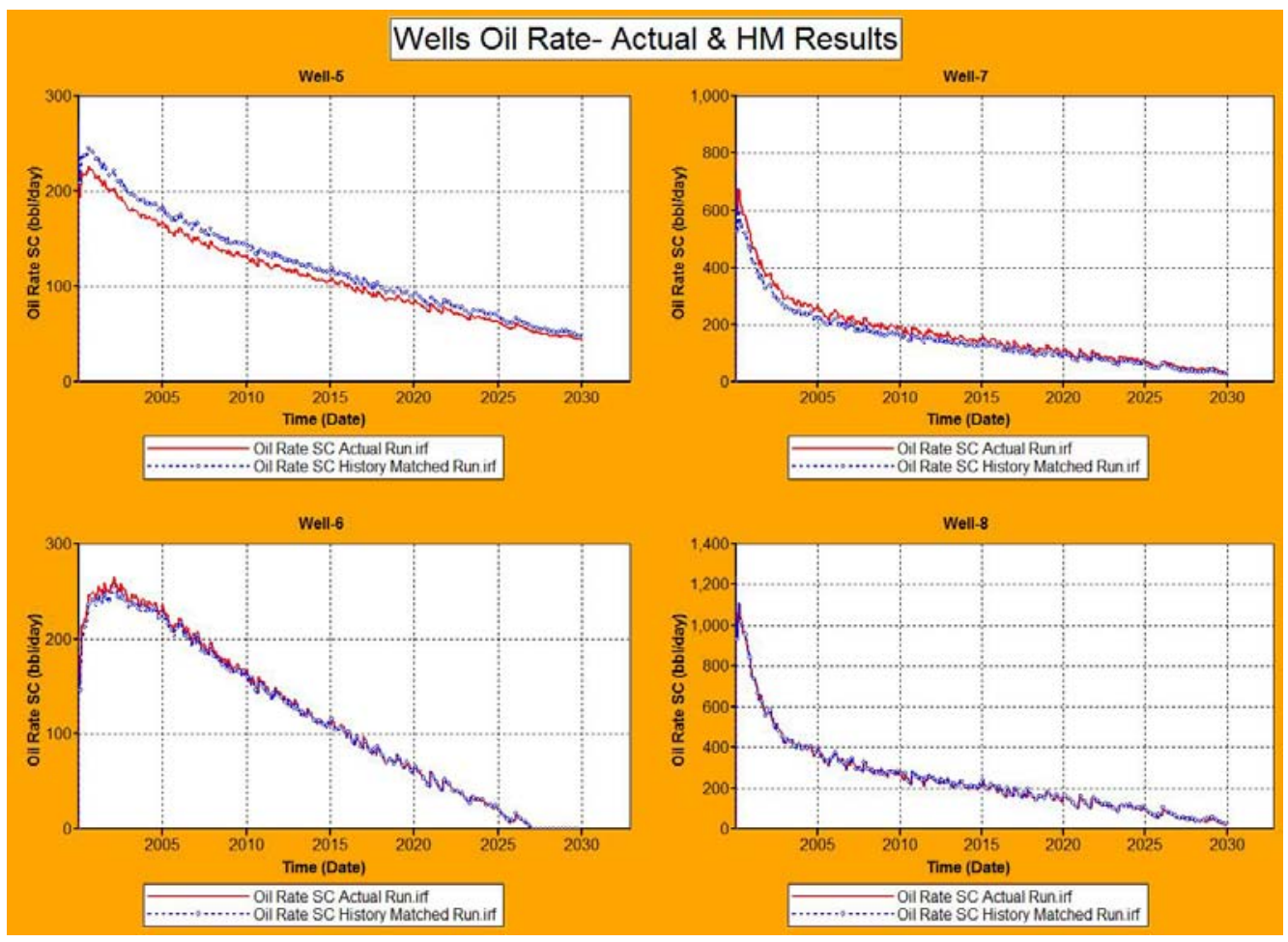

Figure 113- Comparison of oil rate production between actual data and the results coming from simulator after applying the matched permeability map from SRM

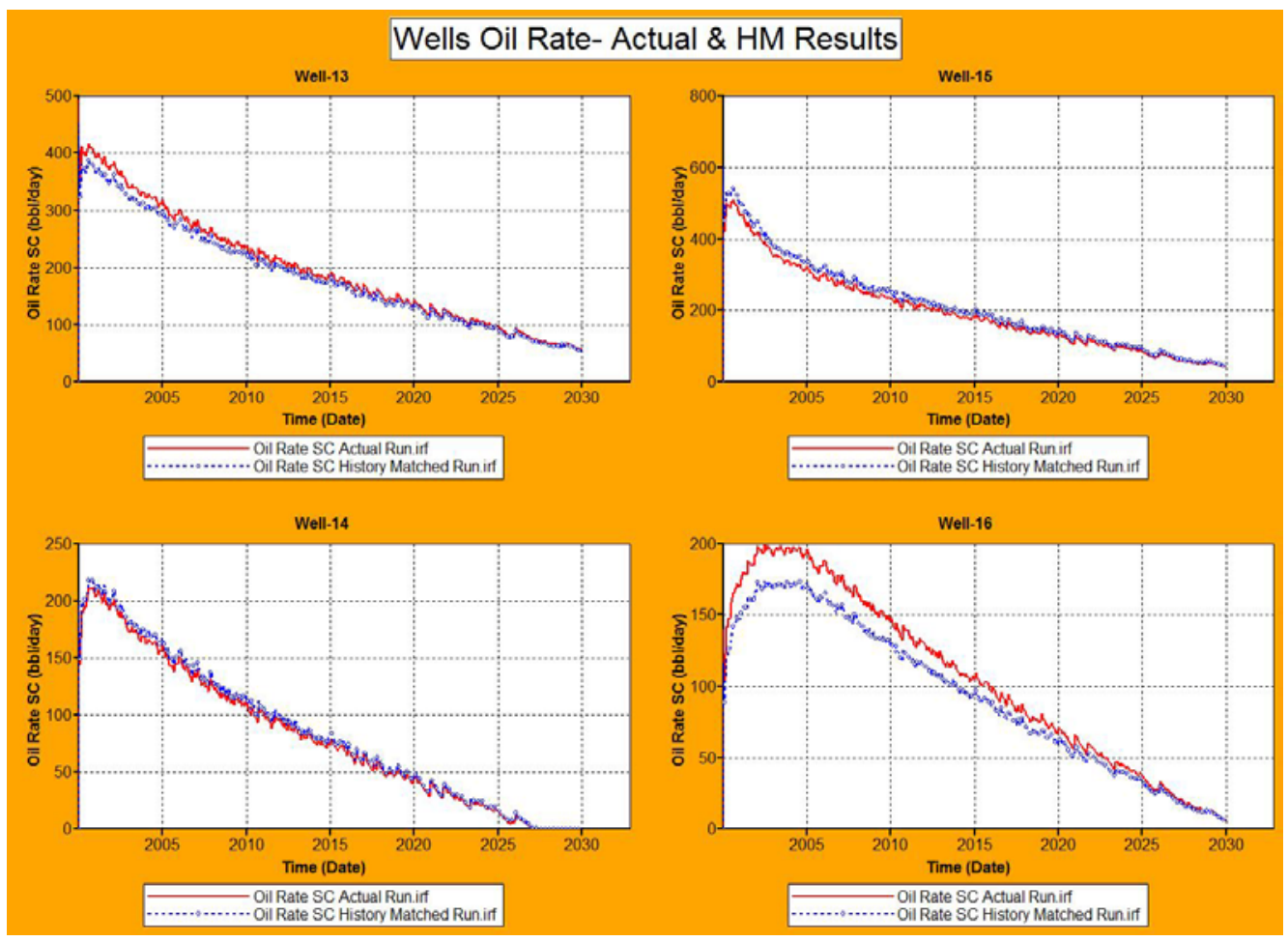

Figure 114- Comparison of oil rate production between actual data and the results coming from simulator after applying the matched permeability map from SRM 


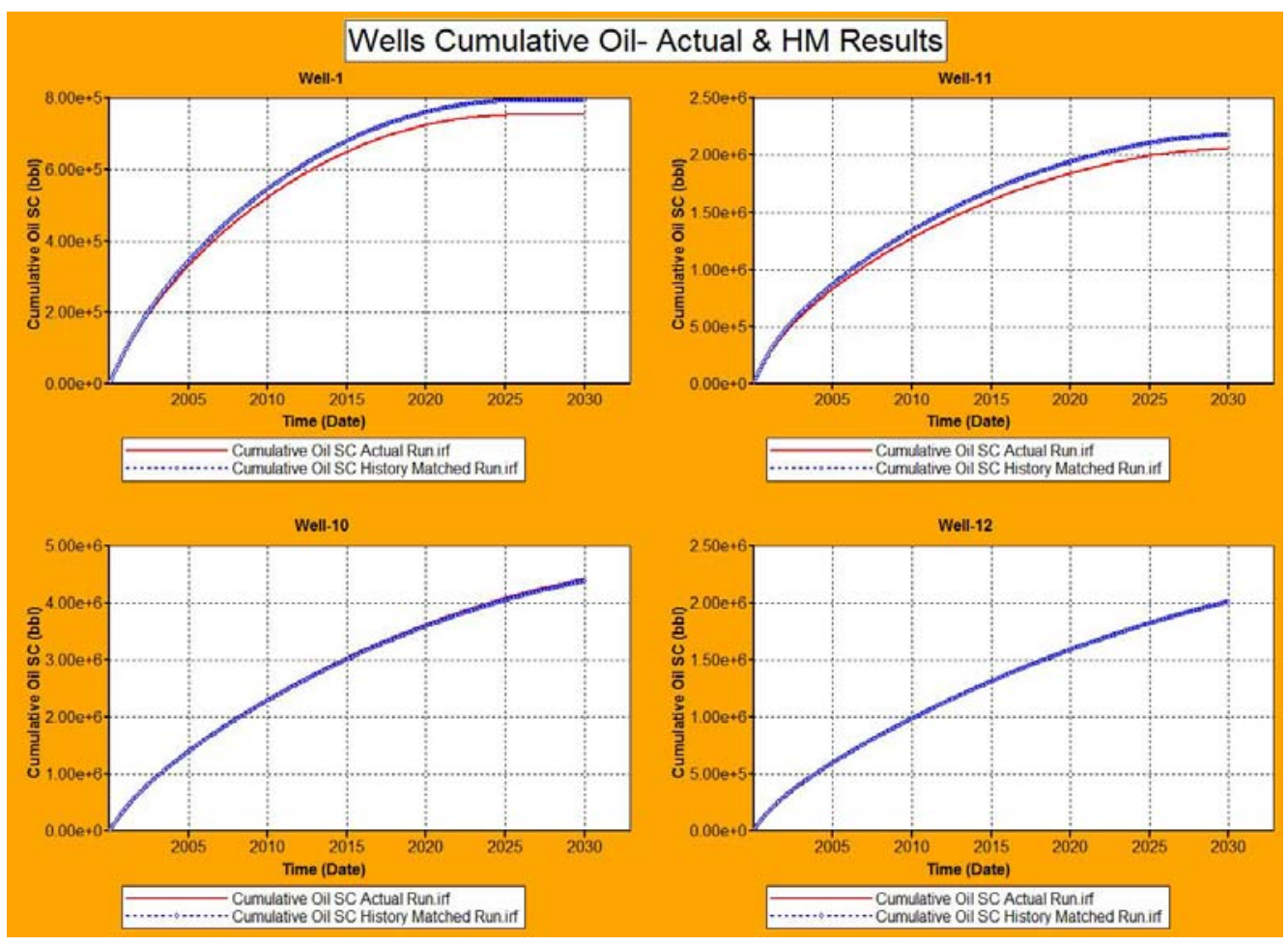

Figure 115-Comparison of cumulative production between actual data and the results coming from simulator after applying the matched permeability map from SRM

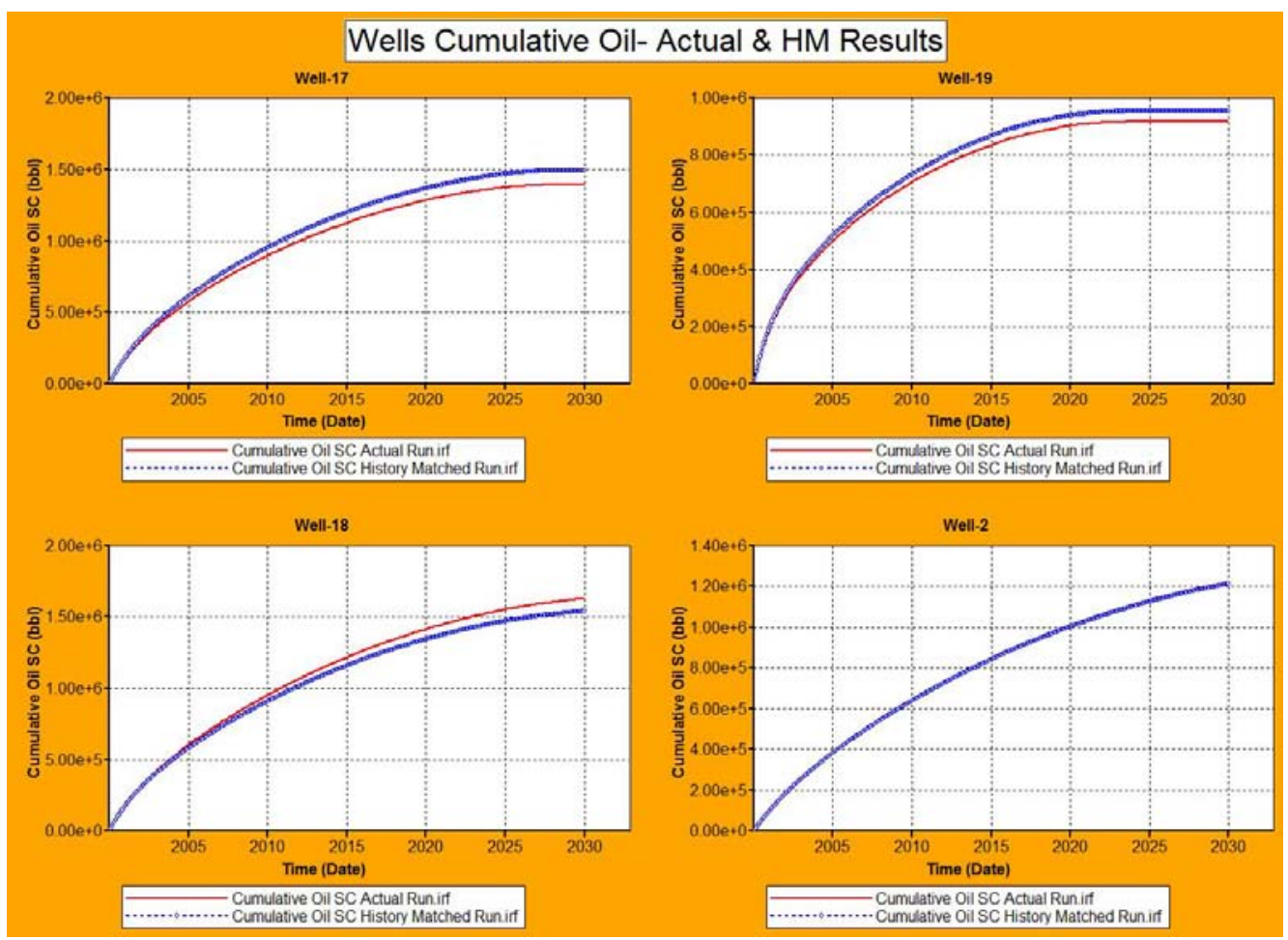

Figure 116-Comparison of cumulative production between actual data and the results coming from simulator after applying the matched permeability map from SRM 


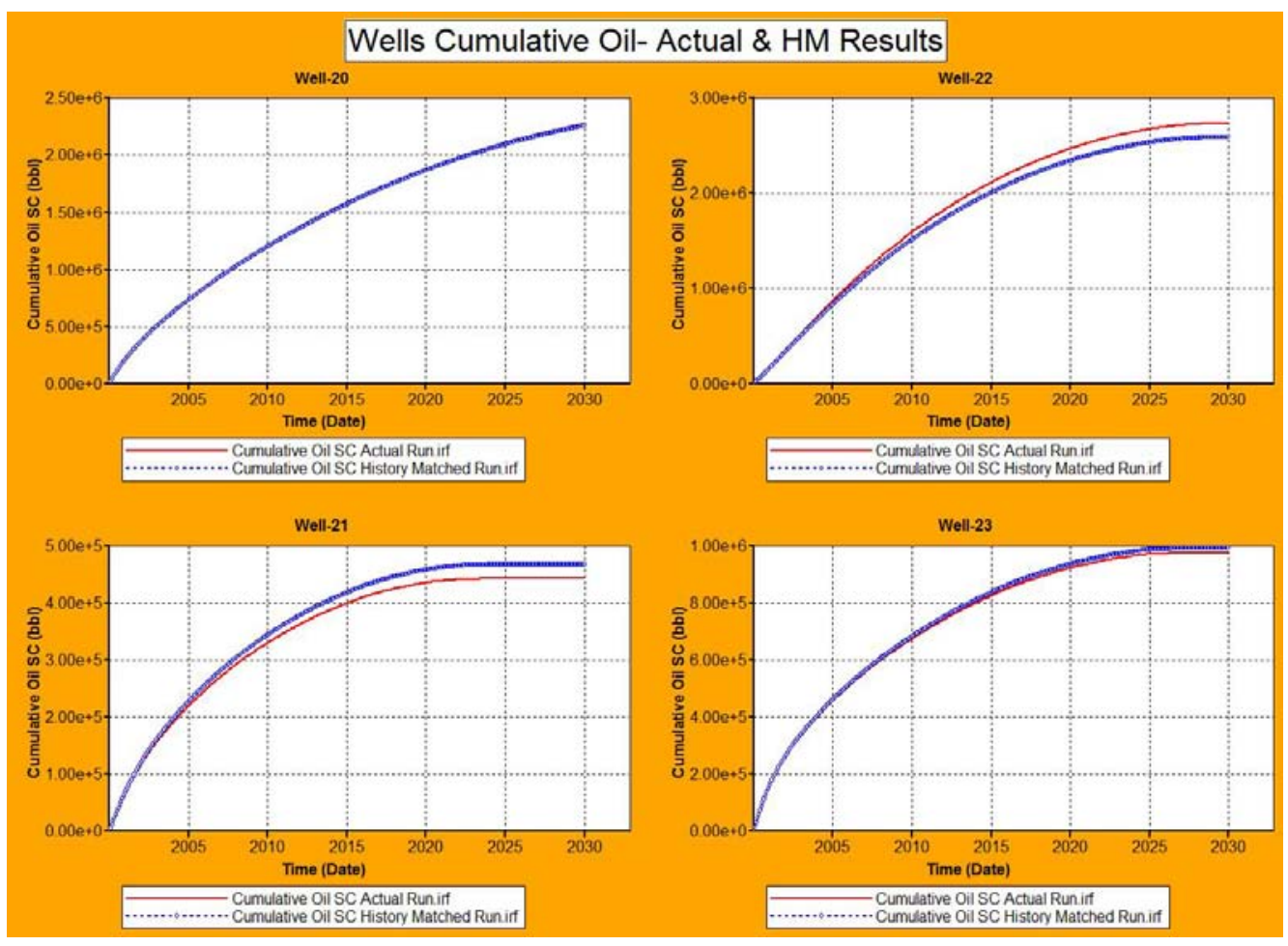

Figure 117-Comparison of cumulative production between actual data and the results coming from simulator after applying the matched permeability map from SRM

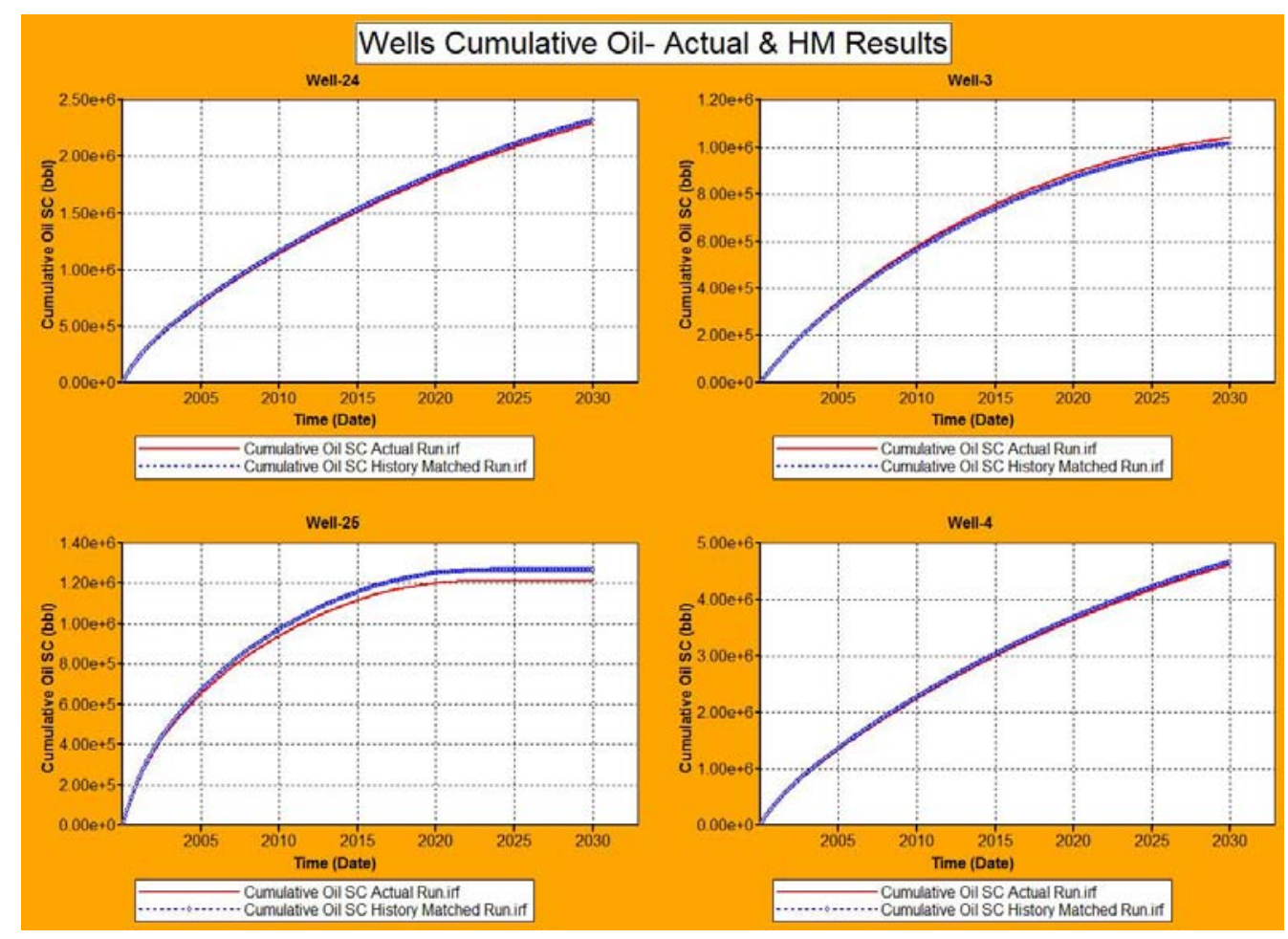

Figure 118-Comparison of cumulative production between actual data and the results coming from simulator after applying the matched permeability map from SRM 


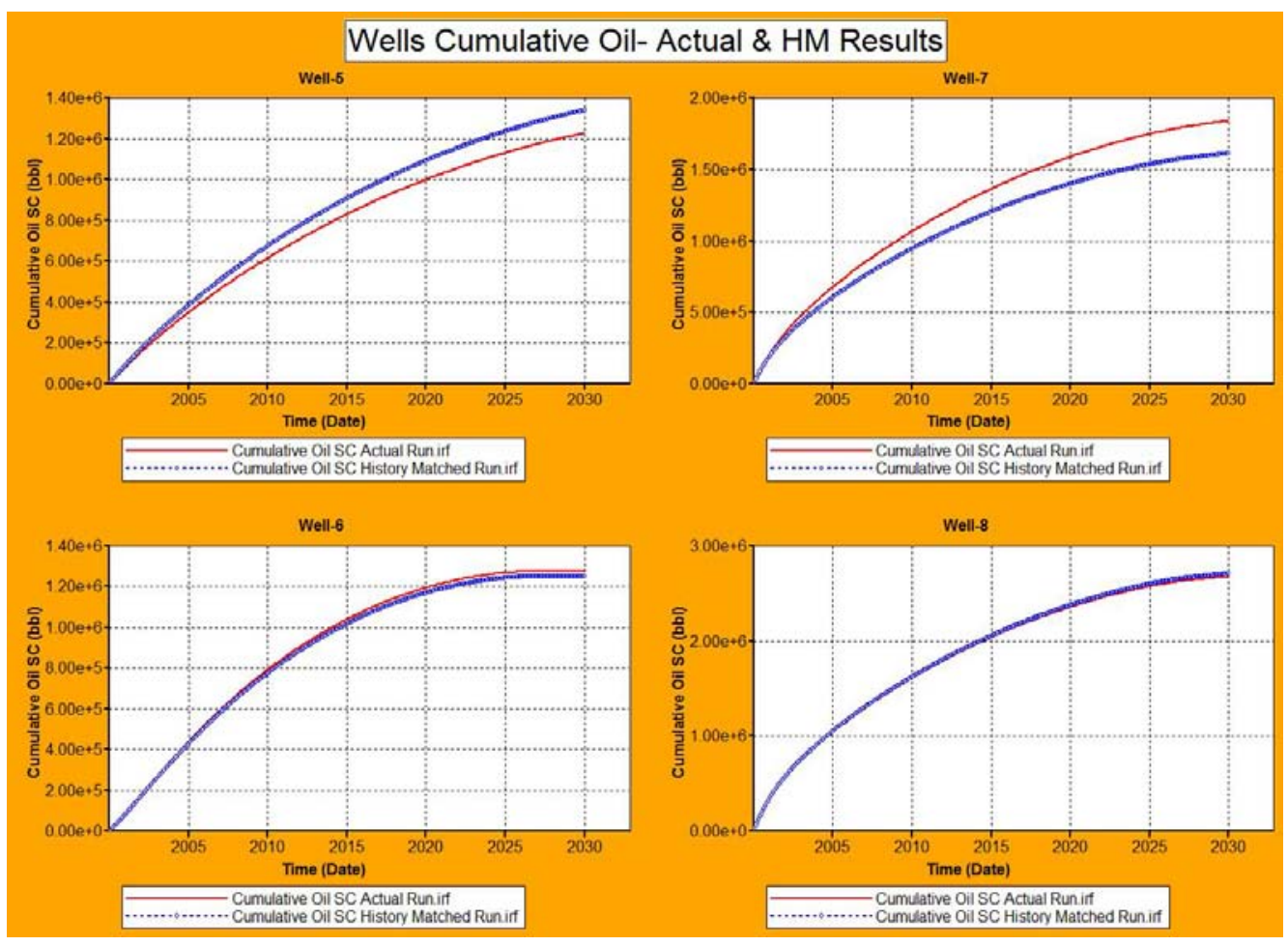

Figure 119-Comparison of cumulative production between actual data and the results coming from simulator after applying the matched permeability map from SRM

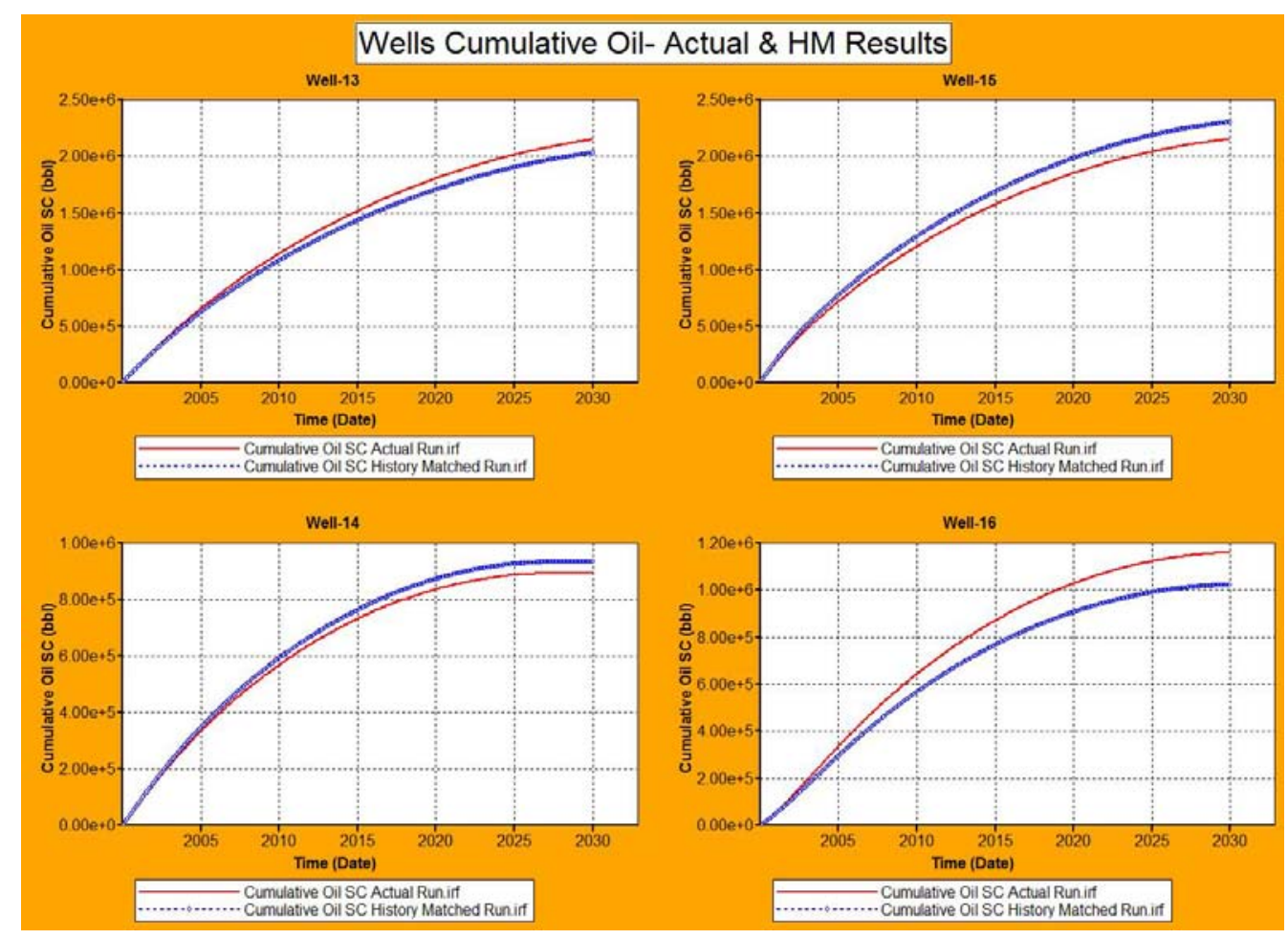

Figure 120-Comparison of cumulative production between actual data and the results coming from simulator after applying the matched permeability map from SRM 